\title{
Pickering Emulsions as Enabling \\ Platforms for Designer Polymer-Based Hybrid Materials
}





\section{PICKERING EMULSIONS AS ENABLING PLATFORMS FOR DESIGNER POLYMER- BASED HYBRID MATERIALS}

Yunchong Zhang 
Graduation Committee:

$\begin{array}{lll}\text { Chairman: } & \text { Prof. dr. J. L. Herek } & \text { University of Twente } \\ \text { Promotors: } & \text { Prof. dr. G. J. Vancso } & \text { University of Twente } \\ & \text { Prof. dr. X. Sui } & \text { Donghua University } \\ \text { Assistant-promotor: } & \text { Dr. J. Duvigneau } & \text { University of Twente } \\ \text { Members: } & \text { Prof. dr. ir. R. G. H. } & \text { University of Twente } \\ & \text { Lammertink } & \\ & \text { Prof. dr. ir. R. ten Klooster } & \text { University of Twente } \\ & \text { Prof. dr. B. Pukánszky } & \begin{array}{l}\text { Budapest University of } \\ \text { Technology and Economics } \\ \end{array} \\ & \text { Prof. dr. ir. R. Tuinier } & \text { Eindhoven University of } \\ & & \text { Technology }\end{array}$

The research described in this Thesis was carried out in the Materials Science and Technology of Polymers (MTP) group (since 2020 Sustainable Polymer Chemistry), MESA+ Institute for Nanotechnology, Faculty of Science and Technology, University of Twente, the Netherlands and in the Key Lab of Science and Technology of Eco-Textile, Ministry of Education, College of Chemistry, Chemical Engineering and Biotechnology, Donghua University, China.

This research was financially supported by the MESA+ Institute for Nanotechnology, University of Twente, by Donghua University, China, and by the China Scholarship Council.

Cover design: Xiaoyue Song

Printed by: IPSKAMP Printing Company, Enschede

ISBN: $\quad 978-90-365-5264-6$

DOI: $\quad 10.3990 / 1.9789036552646$

(C) 2021 Yunchong Zhang, The Netherlands. All rights reserved. No parts of this thesis may be reproduced, stored in a retrieval system or transmitted in any form or by any means without permission of the author. Alle rechten voorbehouden. Niets uit deze uitgave mag worden vermenigvuldigd, in enige vorm of op enige wijze, zonder voorafgaande schriftelijke toestemming van de auteur. 


\title{
PICKERING EMULSIONS AS ENABLING PLATFORMS FOR DESIGNER POLYMER- BASED HYBRID MATERIALS
}

\author{
DISSERTATION
}

to obtain

the degree of doctor at the Universiteit Twente, on the authority of the rector magnificus, prof. dr. ir. A. Veldkamp, on account of the decision of the Doctorate Board to be publicly defended on Thursday 4 November 2021 at 12.45 hours

by

\section{Yunchong Zhang}

born on the 11th of August, 1992

in Hebei, China 
This dissertation has been approved by:

Promotors:

Prof. dr. G. J. Vancso

University of Twente

Prof. dr. X. Sui

Donghua University

Assistant-promotor:

Dr. J. Duvigneau

University of Twente 


\section{Contents}

Chapter 1 General Introduction ............................................................... 1

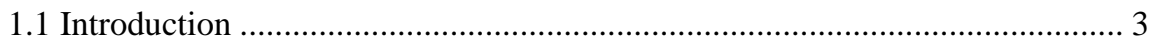

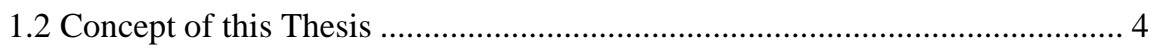

References ................................................................................................. 7

Chapter 2 Pickering Emulsions as Designer Platforms for Polymer-Based Hybrid Materials: Routes to Controlled Structures .................................. 11

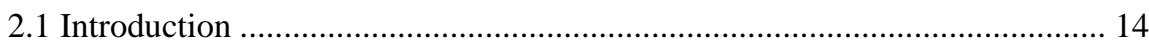

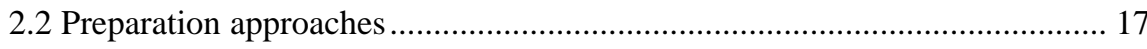

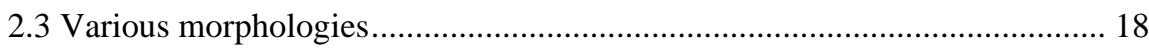

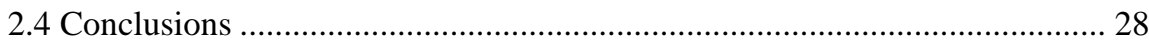

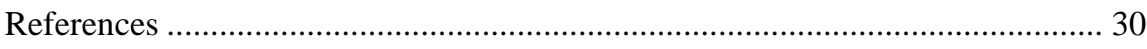

Chapter 3 Poly (lactic acid)/Cellulose Nanocrystal Composites via the Pickering Emulsion Approach: Rheological, Thermal and Mechanical Properties .......... 41

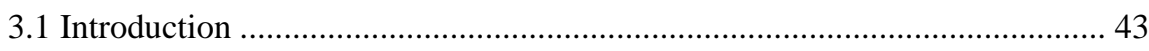

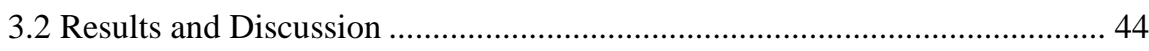

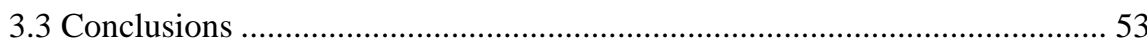

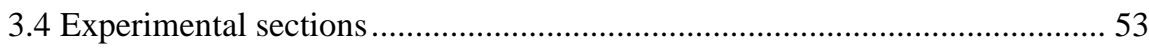

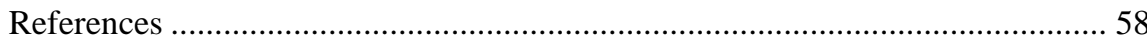

Chapter 4 Making Polymers Colored and Stiffer by Dyed Nanocellulose

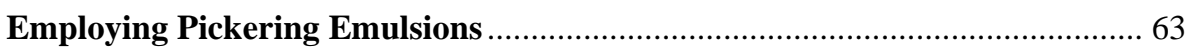

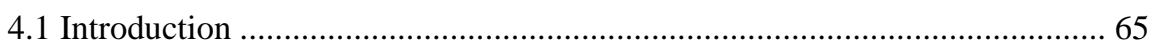

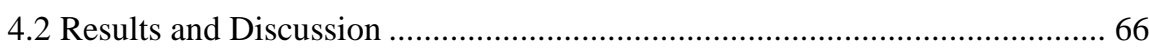

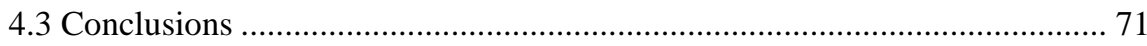


4.4 Experimental sections 72

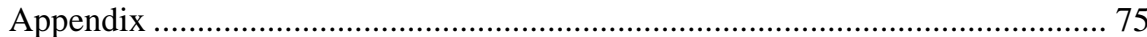

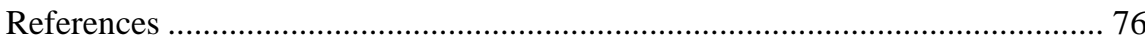

Chapter 5 Enzymatic Degradation of PLA/Cellulose Nanocrystal Composites 79

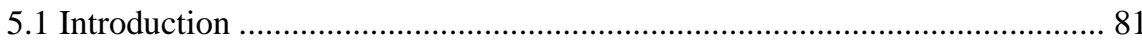

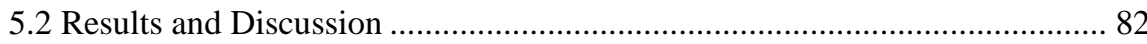

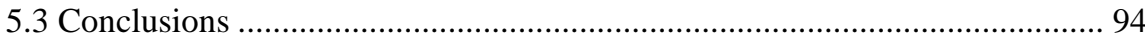

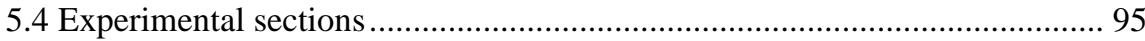

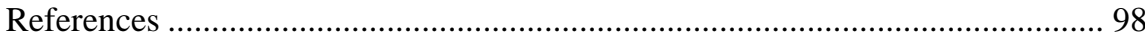

Chapter 6 Foaming of Biodegradable Polylactic Acid/Cellulose Nanocrystal Composites: Pickering Emulsion Templating to Enhance Filler Dispersion and

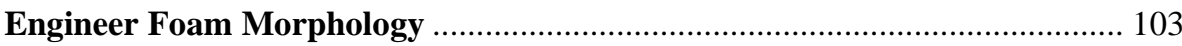

6.1 Introduction ...................................................................................... 105

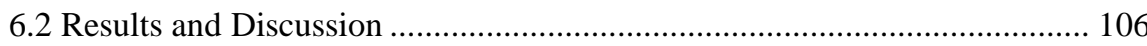

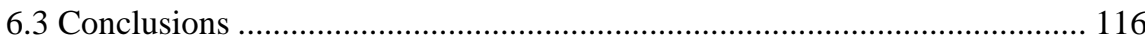

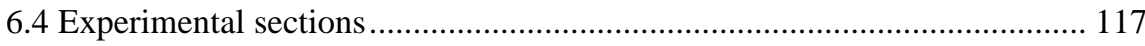

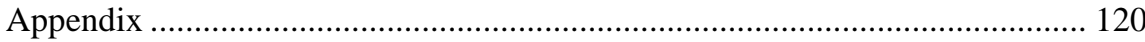

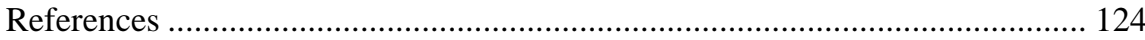

Chapter 7 Highly Stable and Non-Flammable Hydrated Salt-Paraffin ShapeMemory Gels for Sustainable Building Technology ................................... 129

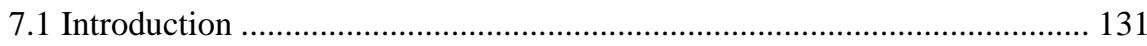

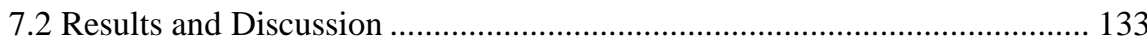

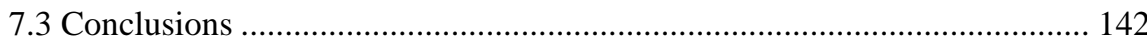

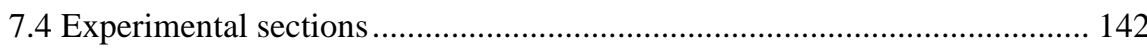

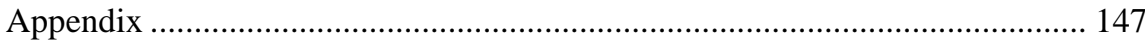


References

Chapter 8 Outlook: Possible Future Trends in Designer Particles as Pickering Emulsifiers 157

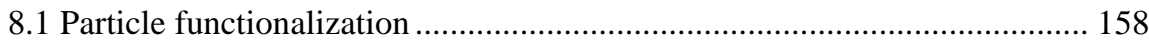

8.2 Combination of different particle types ............................................. 160

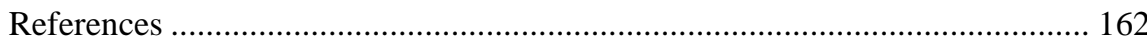

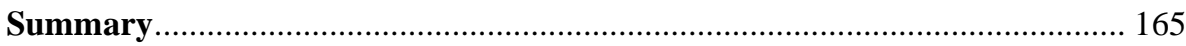

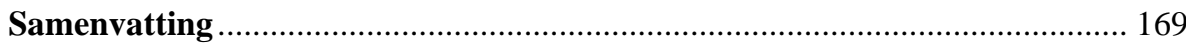

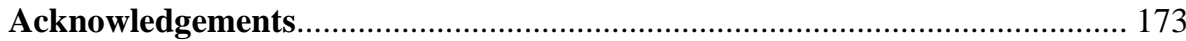

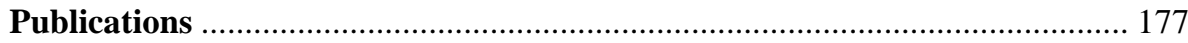

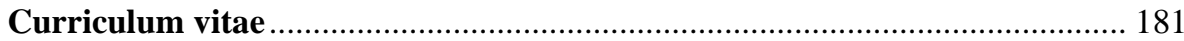





\section{Chapter 1}

General Introduction 


\subsection{Introduction}

Polymer-based hybrid materials have attracted much attention due to their remarkable potential to combine the properties of the individual components to form novel high-performance materials ${ }^{1-2}$. Many strategies have been used to develop polymer-based hybrid materials. In recent years, Pickering emulsion-based templating is considered one of the highly promising platforms for designing polymer-based hybrid materials ${ }^{3-5}$.

Pickering emulsions are stabilized by solid particles (Figure 1.1) instead of surfactants ${ }^{6-8}$. S. U. Pickering is considered the first to report oil-in-water $(O / W)$ emulsions stabilized by solid particles ${ }^{9}$. Actually, Ramsden reported the absorption of solid particles at air-water interface before Pickering ${ }^{10}$. Pickering emulsions are highly stable due to the irreversible adsorption of particles at the interface between the dispersed and continuous phases ${ }^{11-12}$. Different types of Pickering emulsions (waterin-oil (W/O) emulsion, oil-in-water $(\mathrm{O} / \mathrm{W})$ emulsion, and double emulsion) and the combination with other polymer preparation/processing strategies provide opportunities to design various structures of polymer-based hybrid materials, such as microspheres ${ }^{13}$, fibers ${ }^{14}$, films ${ }^{15}$ and porous matrix ${ }^{16}$. Many particles can be used to stabilize emulsions, such as $\mathrm{TiO}_{2}{ }^{17}$, iron oxide ${ }^{18}$, graphene oxide $(\mathrm{GO})^{19}$, and proteins ${ }^{20}$. These Pickering particles provide ample opportunities to prepare multifunctional polymer-based hybrid materials using Pickering emulsion templating.

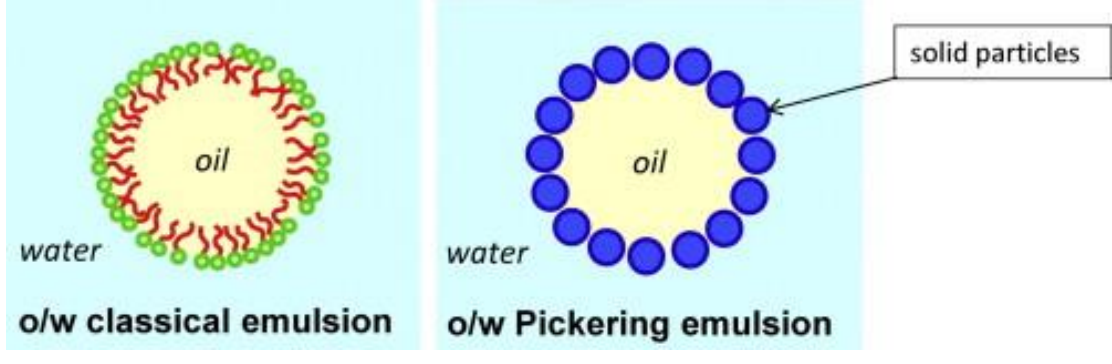

Figure 1.1 Schematic of a classical surfactant-based (left) and a Pickering (right) emulsion ${ }^{21}$.

In addition, biodegradable particles, for example, nanocellulose, can also be used as suitable emulsifiers for Pickering emulsions due to their balanced hydrophilhydrophobe surface character ${ }^{22}$. Figure 1.2 shows an SEM image of nanocellulose particles distributed on the surface of emulsion microspheres. Nanocellulose-based composites have attracted much attention due to their excellent mechanical properties, abundant availability, and easy surface modification ${ }^{23}$. Good dispersion of 
nanocellulose in a polymer matrix is an essential factor to control when preparing composites with optimized properties. Nanocellulose-based Pickering emulsion templates can provide a new strategy to prepare well-dispersed nanocellulose polymer composites. Especially of interest is that hydrophobic polymers can readily be used, while when using conventional blending methods, cellulose particle modification is required for those polymers.

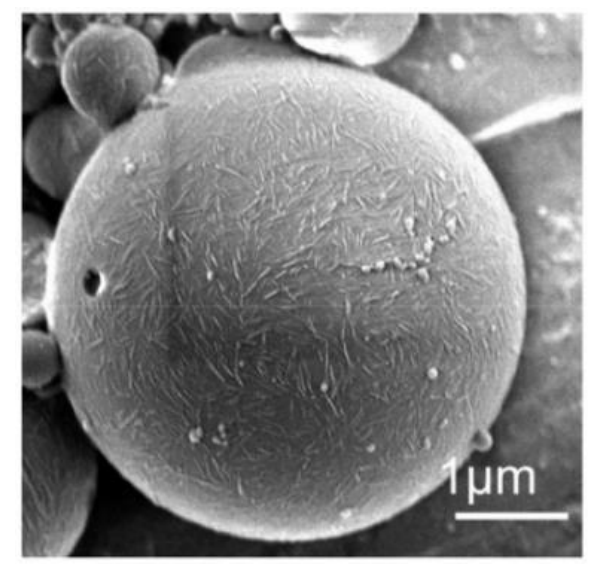

Figure 1.2 A representative SEM image of nanocellulose distribution at the surface of an emulsion microsphere ${ }^{24}$.

However, the structure-property relationships of polymer-based hybrid materials designed by Pickering emulsion platforms are not well studied. In this Thesis, we aim at studying the effect of Pickering emulsion templating on the properties and performance of newly designed functional polymer-based hybrid materials and explore their applications.

\subsection{Concept of this Thesis}

In this Thesis, we use Pickering emulsion methods to engineer heterogeneous polymer materials with enhanced performance. We demonstrate uniform dispersion of nanocellulose in polylactic acid (PLA) matrix as a biobased composite. The performance and properties of these composites are investigated, including the mechanical properties, the thermal properties, the enzymatic degradation properties, and the $\mathrm{CO}_{2}$-foaming behavior. To obtain PLA/CNC (cellulose nanocrystal) composites with a low nanocellulose concentration, Pickering emulsion CNC templated PLA spheres are used as masterbatches that are readily led down by 
conventional melt blending in an extruder. Nanocellulose modification and phase change emulsion phases are used to prepare functional hybrid materials. The resulting phase change emulsion gels are explored as enabling components in building thermal management. The following topics will be presented and discussed in detail in this Thesis.

Chapter 2 provides a literature review covering the general topics discussed in the subsequent chapters. Firstly, we introduce the basics and the formation of Pickering emulsions. Then we discuss how these systems can be used to obtain templates for the fabrication of materials with various morphologies. Secondly, we discuss the uniqueness of Pickering systems to control the morphology to obtain advanced complex and hybrid materials. In addition, we review and summarize recent advances in the preparation approaches and the physical morphologies of polymerbased hybrid materials designed by utilizing Pickering emulsions as a platform technology. Finally, we offer an outlook for future developments of hybrid material templating by Pickering emulsions.

In Chapter 3, the Pickering emulsion solvent evaporation approach is introduced to prepare uniform biodegradable PLA/CNC composite films. The use of nanocellulose is an attractive method to improve the characteristics of biodegradable polymers, but its effectiveness is often hampered by poor particle dispersion. CNCs were evenly dispersed into PLA via the Pickering emulsion approach. The storage modulus and viscosity of the composites increased with the increase of CNC contents due to the excellent CNC dispersion in the PLA matrix. The crystallization and mechanical properties of PLA composites have been improved.

Chapter 4 describes the successful use of Pickering emulsions utilizing nanocellulose that carries covalently immobilized dye molecules as emulsifiers to color PMMA and enhance its mechanical performance. Due to the use of this biobased dye carrier, the materials we describe exhibit an improved "green" character. Pickering emulsion templating yielded a uniform particle dispersion, and as a result, the PMMA composite had high optical transparency. The improved thermal and mechanical properties of PMMA composites are presented.

In Chapter 5, the enzymatic degradation of neat PLA and PLA/CNC composites is described using lipase from Candida rugosa and proteinase $\mathrm{K}$ from Tritirachium album. The effect of CNC content on the PLA degradation rate is investigated. In addition, the impact of $\mathrm{pH}$ and ion concentration are examined, as well. A model, which describes quantitatively the kinetics of enzymatic degradation, is presented and 
applied to describe the kinetics of degradation and denaturation. We show that CNC increased the rate of degradation, and the samples disintegrate very rapidly.

In Chapter 6, the preparation of PLA composites with low CNC contents (below 5 wt.\%) for subsequent $\mathrm{CO}_{2}$ batch foaming is presented. Following Pickering emulsion templating of PLA, the resulting hybrid material was melt blended with pristine PLA to decrease the CNC particle concentration. It is shown that via this Pickering emulsion masterbatch approach, CNC particles are well-dispersed in the PLA matrix without the need for particle surface modification. This was not achieved by direct melt blending of CNCs in PLA before. Finally, neat PLA and PLA/CNC composite films are foamed using a $\mathrm{CO}_{2}$ batch foaming process at relatively mild foaming conditions. The effect of $\mathrm{CNC}$ dispersion, foaming temperature, and foaming time on PLA foaming behavior and foam morphology is elucidated.

Chapter 7 describes highly stable, non-flammable, and shape-memory hydrated salt-paraffin hybrid emulsion gels (EmulGels) prepared via W/O Pickering emulsion templates. EmulGels are specifically designed to enable dual-phase crosslinking, which effectively enhanced its shape-stability, prevented loss of bound water in hydrated salts, decreased the degree of supercooling. This is shown based on leaking and thermal cycling tests of the EmulGels. Finally, the EmulGels are explored as enabling component in building thermal management.

In Chapter 8, an outlook for future functional Pickering particle designs to prepare enhanced polymer-based hybrid materials is given. 


\section{References}

[1] Faustini, M.; Nicole, L.; Ruiz-Hitzky, E.; Sanchez, C. History of organicinorganic hybrid materials: prehistory, art, science, and advanced applications. Advanced Functional Materials 2018, 28 (27), 1704158.

[2] Park, S. S.; Ha, C. S. Hollow mesoporous functional hybrid materials: Fascinating platforms for advanced applications. Advanced Functional Materials 2018, 28 (27), 1703814.

[3] Gonzalez-Ortiz, D.; Pochat-Bohatier, C.; Gassara, S.; Cambedouzou, J.; Bechelany, M.; Miele, P. Development of novel h-BNNS/PVA porous membranes via Pickering emulsion templating. Green Chemistry 2018, 20 (18), 4319-4329.

[4] Kimmins, S. D.; Cameron, N. R. Functional porous polymers by emulsion templating: recent advances. Advanced Functional Materials 2011, 21 (2), 211 225.

[5] Kim, K.; Kim, S.; Ryu, J.; Jeon, J.; Jang, S. G.; Kim, H.; Gweon, D.-G.; Im, W. B.; Han, Y.; Kim, H. Processable high internal phase Pickering emulsions using depletion attraction. Nature Communications 2017, 8 (1), 1-8.

[6] Wu, J.; Ma, G. H. Recent studies of Pickering emulsions: particles make the difference. Small 2016, 12 (34), 4633-4648.

[7] Huo, J.; Marcello, M.; Garai, A.; Bradshaw, D. MOF-polymer composite microcapsules derived from Pickering emulsions. Advanced Materials 2013, 25 (19), 2717-2722.

[8] Jiang, J.; Zhu, Y.; Cui, Z.; Binks, B. P. Switchable Pickering emulsions stabilized by silica nanoparticles hydrophobized in situ with a switchable surfactant. Angewandte Chemie 2013, 125 (47), 12599-12602.

[9] Pickering, S. U. Cxcvi.-emulsions. Journal of the Chemical Society, Transactions 1907, 91, 2001-2021.

[10] Ramsden, W. Separation of solids in the surface-layers of solutions and 'suspensions'. Proceedings of the Royal Society of London 1903, 72, 156-164.

[11] Tambe, D. E.; Sharma, M. M. Factors controlling the stability of colloidstabilized emulsions: I. An experimental investigation. Journal of Colloid and Interface Science 1993, 157 (1), 244-253.

[12] Binks, B.; Lumsdon, S. Pickering emulsions stabilized by monodisperse latex 
particles: effects of particle size. Langmuir 2001, 17 (15), 4540-4547.

[13] Liu, D.; Xue, N.; Wei, L.; Zhang, Y.; Qin, Z.; Li, X.; Binks, B. P.; Yang, H. Surfactant assembly within Pickering emulsion droplets for fabrication of interior-structured mesoporous carbon microspheres. Angewandte Chemie 2018, 130 (34), 11065-11070.

[14] Samanta, A.; Takkar, S.; Kulshreshtha, R.; Nandan, B.; Srivastava, R. K. Facile fabrication of composite electrospun Nanofibrous matrices of poly $(\varepsilon-$ caprolactone)-silica based Pickering emulsion. Langmuir 2017, 33 (32), $8062-$ 8069.

[15] Yang, Z.; Liu, H.; Wu, S.; Tang, Z.; Guo, B.; Zhang, L. A green method for preparing conductive elastomer composites with interconnected graphene network via Pickering emulsion templating. Chemical Engineering Journal 2018, 342, 112-119.

[16] Zhang, Y.; Zhu, G.; Dong, B.; Wang, F.; Tang, J.; Stadler, F. J.; Yang, G.; Hong, S.; Xing, F. Interfacial jamming reinforced Pickering emulgel for arbitrary architected nanocomposite with connected nanomaterial matrix. Nature Communications 2021, 12 (1), 1-9.

[17] Chen, T.; Colver, P. J.; Bon, S. A. Organic-inorganic hybrid hollow spheres prepared from $\mathrm{TiO}_{2}$-stabilized Pickering emulsion polymerization. Advanced Materials 2007, 19 (17), 2286-2289.

[18] Sachdev, S.; Maugi, R.; Kirk, C.; Zhou, Z.; Christie, S. D.; Platt, M. Synthesis and assembly of gold and iron oxide particles within an emulsion droplet; facile production of Core@ Shell particles. Colloid and Interface Science Communications 2017, 16, 14-18.

[19] Zhang, Y.; Zheng, X.; Wang, H.; Du, Q. Encapsulated phase change materials stabilized by modified graphene oxide. Journal of Materials Chemistry A 2014, 2 (15), 5304-5314.

[20] Gao, Z.-M.; Yang, X.-Q.; Wu, N.-N.; Wang, L.-J.; Wang, J.-M.; Guo, J.; Yin, S.W. Protein-based Pickering emulsion and oil gel prepared by complexes of zein colloidal particles and stearate. Journal of Agricultural and Food Chemistry 2014, 62 (12), 2672-2678.

[21] Chevalier, Y.; Bolzinger, M.-A. Emulsions stabilized with solid nanoparticles: Pickering emulsions. Colloids and Surfaces A: Physicochemical and 
Engineering Aspects 2013, 439, 23-34.

[22] Kedzior, S. A.; Gabriel, V. A.; Dubé, M. A.; Cranston, E. D. Nanocellulose in emulsions and heterogeneous water-based polymer systems: A review. Advanced Materials 2020, 2002404.

[23] Kargarzadeh, H.; Huang, J.; Lin, N.; Ahmad, I.; Mariano, M.; Dufresne, A.; Thomas, S.; Gałęski, A. Recent developments in nanocellulose-based biodegradable polymers, thermoplastic polymers, and porous nanocomposites. Progress in Polymer Science 2018, 87, 197-227.

[24] Kalashnikova, I.; Bizot, H.; Cathala, B.; Capron, I. Modulation of cellulose nanocrystals amphiphilic properties to stabilize oil/water interface. Biomacromolecules 2012, 13 (1), 267-275. 


\section{Chapter 2}

\section{Pickering Emulsions as Designer Platforms for Polymer-Based Hybrid Materials: Routes to Controlled Structures}

Polymer-based hybrid materials have attracted great interest in academic research and industry because they can effectively combine the advantages of their different components. Pickering emulsions as designer platforms provide a very efficient and often unappreciated route to prepare polymer-based hybrids. Their applications cover a broad range, including filter media, ion exchange modules, monolithic polymer supports for catalysis, covalent enzyme immobilization, and even scaffolds for tissue engineering. In this review, we first provide an introduction to the basics and formation of Pickering emulsions and then discuss how these systems can be used to obtain templates for the fabrication of materials with various morphologies, such as microspheres, fibers, films and porous matrices. We then discuss the uniqueness of Pickering systems for morphology control and the resulting added value to obtain advanced complex and hybrid materials. We then review and summarize recent advances in the preparation approaches (polymerization and solvent evaporation) and 
physical morphologies (microspheres, fibers, films and porous matrices). Finally, we offer an outlook for future developments.

*The contents of this chapter is in print as a chapter in: Yunchong Zhang, Huan Cheng, Xiang Li, Bijia Wang, Xueling Feng, Zhiping Mao, G. Julius Vancso and Xiaofeng Sui; Pickering Emulsions as Designer Platforms for Polymer-Based Hybrid Materials: Routes to Controlled Structures; Macromolecular Engineering, John Wiley \& Sons Ltd, accepted and in production. 


\section{List of Abbreviations}

O/W: oil-in-water

W/O: water-in-oil

$\mathrm{SiO}_{2}$ : silicon dioxide

$\mathrm{TiO}_{2}$ : titanium dioxide

GO: graphene oxide

CNFs: cellulose nanofibers

CNCs: cellulose nanocrystals

$\mathrm{RC}$ : regenerated cellulose

PEDOT:PSS: poly(3,4-ethylenedioxythiophene):poly(styrenesulfonate)

PLA: polylactic acid

PS: polystyrene

MicroPCMs: phase change microcapsules

MMA: methyl methacrylate

GSA/PANI: graphene-polyaniline

MPMMA: macroporous poly(methyl methacrylate)

SP: sulfonated polystyrene

PCL: poly( $\varepsilon$-caprolactone)

HAp: hydroxyapatite

PLLA: poly(L-lactic acid)

PDLLA: poly(D,L-lactide)

OA: oleic acid

OA-MIONs: oleic acid-coated magnetite iron oxide nanoparticles

Van: vancomycin hydrochloride

PHB: poly- $\beta$-hydroxybutyrate

DMF: dimethylformamide

CNTs: carbon nanotubes

HIPEs: high interphase Pickering emulsions

AAO: artemisia argyi oil

HmPB: hierarchical microporous biocompatible

h- $\mathrm{SiO}_{2}$ : modified $\mathrm{SiO}_{2}$ nanoparticles 


\subsection{Introduction}

Polymer-based hybrid materials are formed by a suitable combination of two or more components. A synergistic combination of components with controlled structures of the individual constituents across the length scales can be utilized to enhance mechanical strength, improve chemical stability, and regulate optical, anticorrosive, magnetic, electrical and thermal properties ${ }^{1-2}$. In view of the challenging science and promising applications, Pickering emulsions as designer platforms for polymer-based hybrid materials have become an emerging research topic in recent years ${ }^{3-5}$.

Pickering emulsions are stabilized by solid particles in place of surfactants ${ }^{6-8}$. They are relatively stable due to the irreversible adsorption of particles at the interface between the dispersed and continuous phases ${ }^{9-10}$. The use of biosolid stabilizers makes Pickering emulsions more attractive for food and biomaterials ${ }^{11-13}$. Functional particles can impart Pickering emulsion functionality and expand its application ${ }^{14}$.

The wettability of the stabilizing particles determines into which phase the particles will be preferentially solubilized. If the particles are relatively hydrophilic, they will be more soluble in the aqueous phase than in the oily phase and thus will preferentially form oil-in-water $(\mathrm{O} / \mathrm{W})$ emulsions ${ }^{15}$. On the other hand, hydrophobic particles tend to form water-in-oil (W/O) emulsions ${ }^{16}$. This property could be used as an additional handle for tuning the properties of the final polymeric composites. The positions of solid particles at the oil-water interface for two types of contact angles are shown in Figure 2.1. 


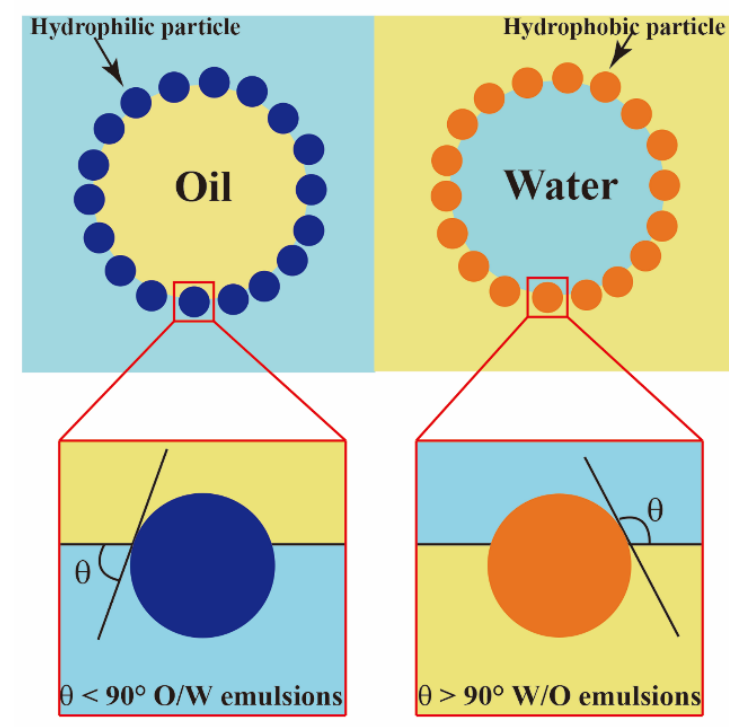

Figure 2.1 Schematic illustration of the formation of $\mathrm{O} / \mathrm{W}$ (left) and W/O (right) emulsions stabilized by solid particles with drasticallydifferent wettabilities.

Another key point is that the emulsified structure of these materials not only provides morphology control for the whole system, but the properties of the emulsifying particles can also have an essential positive effect on the overall properties of the hybrids. Various solid particles, such as silicon dioxide $\left(\mathrm{SiO}_{2}\right)^{17}$, titanium dioxide $\left(\mathrm{TiO}_{2}\right)^{18}$, iron oxide ${ }^{19}$, nanocellulose ${ }^{20}$, graphene oxide $(\mathrm{GO})^{21}$, and proteins $^{22}$, have been used as Pickering stabilizers. These particles stay at the oil-water interface in emulsions, thus functionalizing the interface of the resulting polymer and endowing novel properties to the obtained composites.

Polymerization and solvent evaporation of polymer solutions are two approaches to prepare hybrid materials via the Pickering emulsion approach. In the process of polymerization, monomers are used in the Pickering emulsion, followed by polymerization to achieve the final structure. During the solvent evaporation process, a polymer solution is employed, and following structure formation within the emulsion, the solvent is eventually evaporated.

Most of the current reviews on hybrid materials obtained via Pickering emulsions focus on the polymerization approach, and we provide an overview of the most recent review papers in Table 2.1. While the importance of solvent evaporation has become increasingly prominent, no single comprehensive and comparative report exists 
focusing on both preparation approaches ${ }^{3-4,23-25}$.

Table 2.1 Recent review papers focusing on polymer-based hybrid materials obtained via Pickering emulsion.

\begin{tabular}{|c|c|c|c|c|}
\hline Generic focus & Review title & Author(s) & Year & Reference \\
\hline Hybrid foams & $\begin{array}{l}\text { Hybrid foams, colloids } \\
\text { and beyond: From } \\
\text { design to applications }\end{array}$ & $\begin{array}{l}\text { Rénal } \\
\text { Backov et } \\
\text { al }\end{array}$ & 2011 & 26 \\
\hline Nanoparticles & $\begin{array}{c}\text { Pickering-type } \\
\text { stabilized nanoparticles } \\
\text { by heterophase } \\
\text { polymerization }\end{array}$ & $\begin{array}{l}\text { Ulrich } \\
\text { Ziener et al }\end{array}$ & 2013 & 27 \\
\hline $\begin{array}{l}\text { Biomedical } \\
\text { applications }\end{array}$ & $\begin{array}{c}\text { Recent Studies of } \\
\text { Pickering Emulsions: } \\
\text { Particles Make the } \\
\text { Difference }\end{array}$ & $\begin{array}{l}\text { Guang-Hui } \\
\text { Ma et al }\end{array}$ & 2016 & 3 \\
\hline Foams & $\begin{array}{c}\text { Biopolymer-based } \\
\text { particles as stabilizing } \\
\text { agents for emulsions } \\
\text { and foams }\end{array}$ & $\begin{array}{l}\text { Eric } \\
\text { Dickinson }\end{array}$ & 2017 & 28 \\
\hline $\begin{array}{l}\text { Hybrid } \\
\text { materials }\end{array}$ & $\begin{array}{c}\text { Development of Novel } \\
\text { Materials from } \\
\text { Polymerization of } \\
\text { Pickering Emulsion } \\
\text { Templates }\end{array}$ & $\begin{array}{l}\text { Bo-Geng } \\
\text { Li et al }\end{array}$ & 2017 & 23 \\
\hline $\begin{array}{l}\text { Conducting } \\
\text { polymer } \\
\text { nanocomposit } \\
\text { es }\end{array}$ & $\begin{array}{l}\text { Pickering emulsion- } \\
\quad \text { polymerized } \\
\text { conducting polymer } \\
\text { nanocomposites and } \\
\text { their applications }\end{array}$ & $\begin{array}{l}\text { Hyoung Jin } \\
\text { Choi et al }\end{array}$ & 2017 & 4 \\
\hline
\end{tabular}


In recent years, our group has been actively preparing polymer-based hybrid materials via Pickering emulsion templating. Various particles, including cellulose nanofibers $(\mathrm{CNFs})^{29}$, cellulose nanocrystals $(\mathrm{CNCs})^{30}$, regenerated cellulose $(\mathrm{RC})^{31}$, lignin nanoparticles ${ }^{32}$ and regenerated $\operatorname{chitin}^{33}$, have been used to prepare polymerbased hybrid films followed by a solvent evaporation approach. As examples of the polymerization route, we mention our reports on polystyrene hybrid films ${ }^{34}$, phase change microcapsules ${ }^{35-36}$ and organohydrogels.

In the following sections, we will review and summarize recent advances in polymer-based hybrid materials from Pickering emulsions with a focus on structure formation and morphology characterization.

\subsection{Preparation approaches}

As mentioned earlier, two general methods, namely, polymerization and solvent evaporation, are employed to obtain structured hybrid materials from Pickering emulsions. Starting with monomers brings additional dimensions for structure and property tuning, while the solvent evaporation approach offers easy processability and well-defined polymer composition, as no chemical reactions are involved. Here, we provide a brief summary of the main features of the various preparation options.

The polymerization approach can be further categorized as Pickering emulsion polymerization $^{37}$, Pickering mini-emulsion polymerization ${ }^{38}$, and Pickering suspension polymerization ${ }^{39}$. The processes are similar to traditional emulsion polymerization and suspension polymerization except that solid particles are used instead of surfactants, which provide superior stabilization of the dispersed phase. The Pickering emulsion contains a continuous phase and a dispersed phase. If the monomer is present in the dispersed phase, hybrid materials featuring spherical domains can be prepared ${ }^{40}$. If the monomer is present in the continuous phase, then porous materials can be fabricated ${ }^{41}$. However, if monomers exist in both phases in the Pickering emulsion, organohydrogels can be prepared ${ }^{42-44}$.

With the solvent evaporation approach, polymer solutions are typically used as the oily phase. As the solvent gradually evaporates, the polymer separates out and structures the skeleton of the polymeric hybrid materials. The solvent evaporation approach has been employed for a wide range of biocompatible compositions. Microspheres can be prepared via the $\mathrm{O} / \mathrm{W}$ Pickering route ${ }^{45}$, which could be hotpressed to make composite films ${ }^{32}$. If $\mathrm{W} / \mathrm{O}$ systems are utilized, porous polymer materials can be obtained ${ }^{46}$. In addition, core-shell fibers can be fabricated by 
combining the Pickering emulsion solvent evaporation approach with electrospinning ${ }^{47}$.

\subsection{Various morphologies}

\subsubsection{Microspheres}

Polymer-based microspheres have attracted considerable interest due to their fascinating applications in fields such as fabric printing ${ }^{48}$, contaminant removal ${ }^{49}$, thermal management ${ }^{50}$, and biomedical applications ${ }^{51}$. Various functional particles, such as $\mathrm{TiO}_{2}{ }^{52}$, graphene ${ }^{53}, \mathrm{ZnO}^{54}$, and $\mathrm{Fe}_{3} \mathrm{O}_{4}{ }^{55}$, have been used to prepare functional polymeric hybrid microspheres. Different morphologies, such as solid microspheres (microbeads), microcapsules and porous microspheres, are easily accessible.

\section{Polymerization approach}

Bon and coworkers fabricated poly(methyl methacrylate) latex armored with silica nanoparticles via Pickering emulsion polymerization ${ }^{56}$. Complex multilayered nanocomposite polymer colloids can be obtained by adding a second conventional seeded emulsion polymerization step. TEM images of the resulting hybrid microspheres are provided in Figure 2.2.

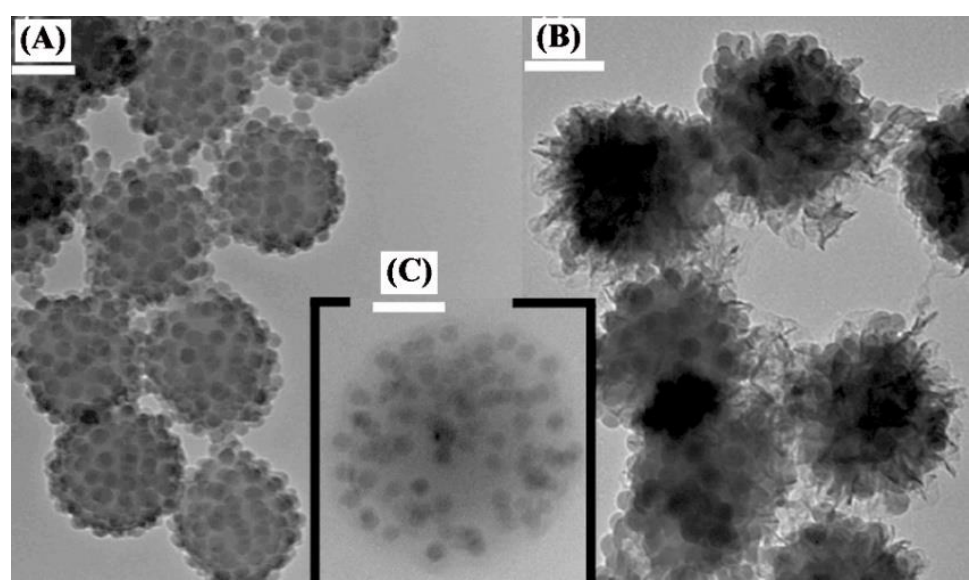

Figure 2.2 TEM images (scale bar $=100 \mathrm{~nm}$ ) of $(\mathrm{A})$ poly (methyl methacrylate) latex armored with silica nanoparticles obtained by Pickering emulsion polymerization. Multilayered nanocomposite polymer colloids with (B) a "hairy" outer layer of poly(acrylonitrile) and (C) a soft shell of poly(n-butyl acrylate). Reproduced with permission ${ }^{56}$. Copyright 2008, American Chemical Society. 
Functional microspheres can be prepared using functionally stabilizing particles. Rojas and coworkers used $\mathrm{CNC}-\mathrm{CoFe}_{2} \mathrm{O}_{4}$ as the Pickering stabilizer to fabricate magnetic PS microbeads with shells reinforced by $\mathrm{CNCs}^{57}$. The microbeads were used as immobilization supports to remove a dye from water. Yu and coworkers prepared pH-responsive microcapsules using perfluorooctyltriethoxysilane-loaded silica nanocapsules as stabilizers, which can be applied in multifunctional textiles ${ }^{58}$.

Pickering emulsion polymerization can also be used to prepare double-shelled magnetic hybrid capsules for controlled drug delivery ${ }^{59}$. Hydrophobic $\mathrm{Fe}_{3} \mathrm{O}_{4}$ nanoparticles were dispersed into the inner polymer shell, and modified $\mathrm{SiO}_{2}$ nanoparticles were attached to the surfaces of microcapsules to form an outer shell. The $\mathrm{Fe}_{3} \mathrm{O}_{4}$ particles were found to have a secondary enhancement effect on the capsules.

Microcapsules can be facilely prepared via Pickering emulsion templates. The barrier constructed by the self-assembly of solid particles can serve as a framework to form a strengthened shell wall, reinforcing the resistance of the capsule and preserving the core materials from deactivation by ambient hazards. Microcapsules loaded with a healing reagent (isophorone diisocyanate, IPDI) were prepared from an $\mathrm{O} / \mathrm{W}$ Pickering emulsion stabilized by lignin nanoparticles ${ }^{60}$. Multilayered composite microcapsules were efficiently formed with a total IPDI encapsulation efficiency of $87 \%$ and exhibited excellent resistance properties towards heat, moisture, and aqueous solutions. Our group used lignin nanoparticles as stabilizers to prepare MicroPCMs ${ }^{35-}$ 36. The resulting MicroPCMs exhibited a well-defined core-shell spherical morphology. Additionally, the MicroPCMs had high enthalpy and thermal stability and can be applied in the electronics and construction industries.

Hollow spheres can be obtained when no core material is present. Hollowstructured graphene-polyaniline spheres (GSA/PANI) were prepared using sulfonated graphene (GSA) as the Pickering stabilizer ${ }^{61}$. The size, as well as the electrochemical properties, of these spheres could be adjusted by varying the amphipathicity, concentration of GSA and the oil-to-water volume ratios. The GSA/PANI spheres exhibited superior performance as supercapacitors compared to the conventional stacked two-dimensional GSA/PANI composites. This unique hollow structure provides a larger accessible surface and shortens the change transfer path.

By removing solid particles, hollow porous microspheres can be fabricated. Kim and coworkers prepared plasmonic Janus hollow porous microspheres by removing $\mathrm{SiO}_{2}$ particles after Pickering emulsion polymerization followed by metal deposition ${ }^{62}$. 
An illustration of this process is shown in Figure 2.3A. In addition to $\mathrm{W} / \mathrm{O}$ or $\mathrm{O} / \mathrm{W}$ Pickering templates, air-in-water Pickering emulsion templates are also an efficient approach to prepare hollow porous microspheres. Pan and coworkers used a $\mathrm{CO}_{2}$-inwater template stabilized by $\mathrm{SiO}_{2}$ particles to generate hollow porous microspheres by removing particles after interfacial polymerization ${ }^{63}$. These microspheres were used as sorbents for fast and highly selective uranium extraction after modification with polyethylene polyamine.

Wang and coworkers prepared a double Pickering emulsion using hydrophobic $\mathrm{SiO}_{2}$ nanoparticles and $\mathrm{Fe}_{3} \mathrm{O}_{4}$ nanoparticles as stabilizers, from which nanocomposite polystyrene microspheres with a multihollow structure were obtained (Figure 2.3B) ${ }^{64}$. The number and structure of pores in the multihollow PS microspheres could be tuned by simply changing the volume ratio of $\mathrm{W}: \mathrm{O}$ in the double emulsion template. This kind of hybrid multihollow polymer microsphere is expected to have wide potential applications in materials science and biotechnology.

Structured and molecularly imprinted multicore rattle-type microspheres of poly(4-vinylpyridine) have been reported, where $\mathrm{SiO}_{2}$ nanoparticles were used as the only Pickering stabilizer ${ }^{65}$. SEM images of these multicore rattle-type microspheres are shown in Figure 2.3C. The void inside the sphere was induced by the phase separation driven by the polymerization and tailored by the interface interaction. The imprinting template of bisphenol A served as the heterogeneous nuclei, whose superimposition on polymerization induced phase separation, creating opportunities for the formation of multicores encapsulated in the shell. 
(A)

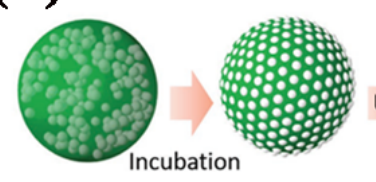

UV

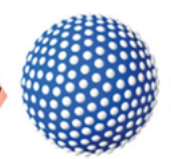

$\mathrm{HF}$

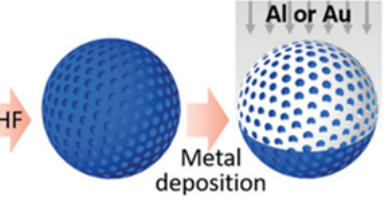

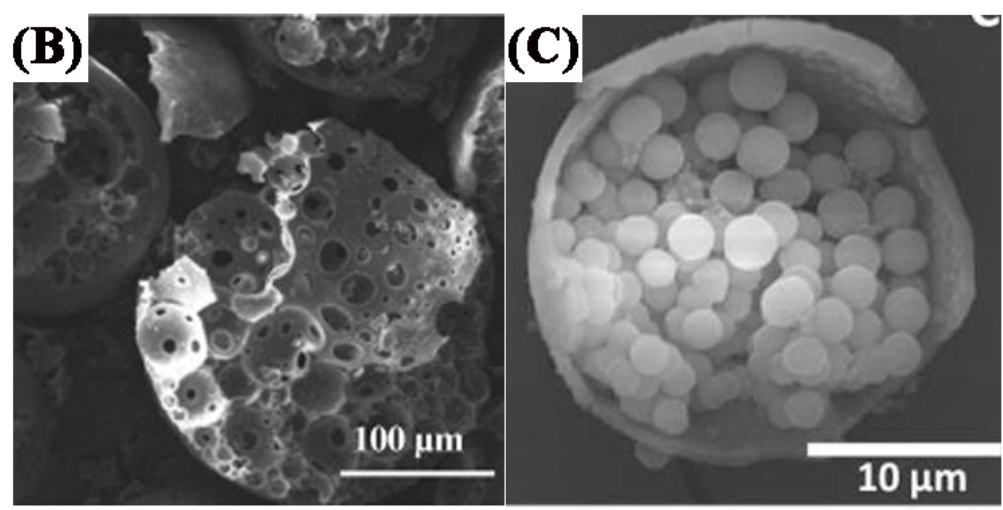

Figure 2.3 (A) Illustration of the plasmonic microsphere fabrication steps. Reproduced with permission.$^{62}$ Copyright 2020, Wiley publishers. SEM images of (B) Multihollow microspheres. Reproduced with permission. ${ }^{64}$ Copyright 2013, Elsevier. (C) Multicored microcapsules. Reproduced with permission. ${ }^{65}$ Copyright 2018, Elsevier.

\section{Solvent evaporation approach}

Hydroxyapatite (HAp) nanoparticle-armored poly( $\varepsilon$-caprolactone) (PCL) microspheres were fabricated via the solvent evaporation approach ${ }^{66}$. A PCL solution in $\mathrm{CH}_{2} \mathrm{Cl}_{2}$ was used as the oil phase, and the HAp nanoparticles were used as the solid emulsifier.

HAp was also used to make multihollow HAp/poly(l-lactic acid) (PLLA) nanocomposite microspheres. A double Pickering emulsion of $\mathrm{W} / \mathrm{O}\left(\mathrm{CH}_{2} \mathrm{Cl}_{2}\right.$ solution of PLLA)/W was generated using two kinds of HAp with different wettabilities ${ }^{67}$. In both cases, the interaction between the ester carbonyls on the polymers and HAp nanoparticles at the oil-water interfaces played a crucial role. These composites are expected to be used as drug delivery carriers and scaffold particles in medical fields. Microcapsules of poly(lactic-co-glycolic acid) (PLGA)-coated with magnetic iron oxide $\left(\mathrm{Fe}_{2} \mathrm{O}_{3}\right)$ nanoparticles were also fabricated for the delivery of hydrophobic $\operatorname{drugs}^{68}$.

MicroPCMs can also be easily prepared by a solvent evaporation approach. For 
example, our group employed cellulose nanocrystals as the stabilizer and poly(methyl methacrylate) solution in $\mathrm{CH}_{2} \mathrm{Cl}_{2}$ as the oil phase ${ }^{69}$. The MicroPCMs have good thermal stability and a melting enthalpy of up to $150.8 \mathrm{~J} / \mathrm{g}$.

\subsubsection{Fibers}

To date, only the solvent evaporation approach has been reported for preparing hybrid fibers templated with Pickering emulsions. Specifically, core-shell structured fibers were prepared by electrospinning of either a $\mathrm{W} / \mathrm{O}$ or $\mathrm{O} / \mathrm{W}$ emulsion ${ }^{70}$. These fibers were shown to have high potential for use as wound dressings and in drug delivery and bone tissue engineering ${ }^{71-73}$.

Laccase from Trametes versicolor was encapsulated in situ into poly(D,L-lactide) (PDLLA) from the corresponding W/O $\left(\mathrm{CH}_{2} \mathrm{Cl}_{2}\right.$ solution of PDLLA) stabilized by PEO-PPO-PEO (F108) ${ }^{74}$. The core-shell structure of the microfibers and the presence of laccase in the microfibers were shown by laser confocal scanning microscopy micrographs, as shown in Figure 2.4A. The porous morphology of the microfibers was confirmed by SEM, as shown in Figure 2.4B.
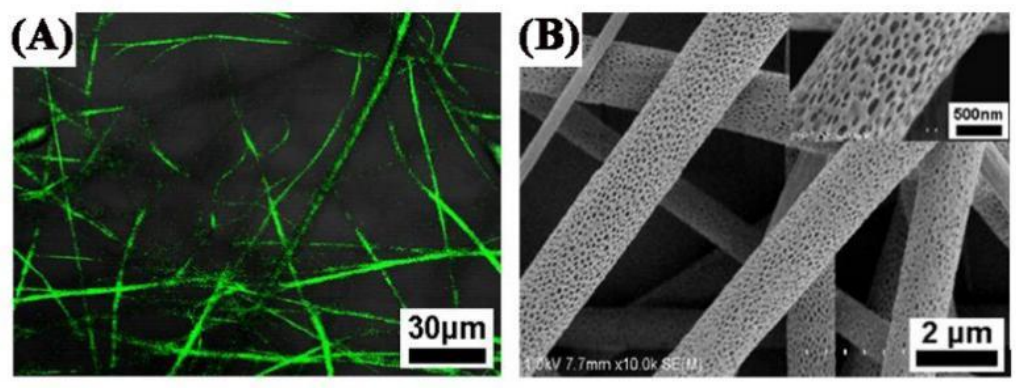

Figure 2.4 (A) Laser confocal microphotograph of core-shell structured PDLLA/F108-laccase (labeled with FITC) composite microfibers. (B) SEM image of the composite microfibers. Reproduced with permission ${ }^{74}$. Copyright 2010, Elsevier.

As shown in the scheme in Figure 2.5A, vancomycin hydrochloride (Van)-loaded core/shell nanofibers were prepared from a W (aqueous Van solution)/O (PLA solution in chloroform) Pickering emulsion stabilized by oleic acid (OA)-coated magnetite iron oxide nanoparticles (OA-MIONs) ${ }^{47}$. The fibers had a core-shell structure with diameters less than $1 \mu \mathrm{m}$ (Figure 2.5B). Membranes made from these nanofibers showed good tensile strength and resilience and excellent antibacterial properties due to the combinational antibacterial activities of OA-MIONs and aqueous Van (Figure 
2.5C).

(A)

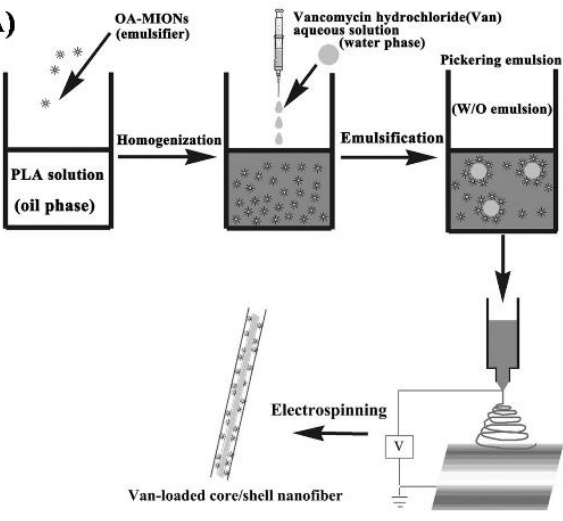

(B)

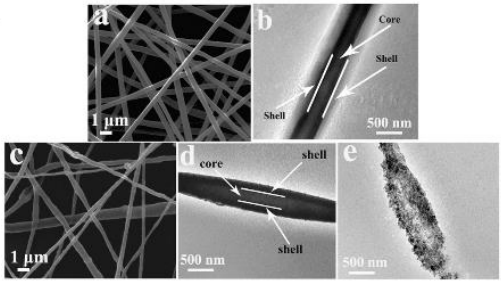

(C)

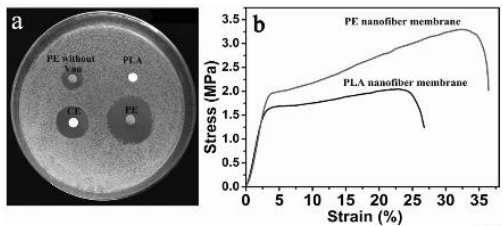

Figure 2.5 (A) Preparation of Van-loaded core/shell nanofiber via Pickering emulsion electrospinning. (B) SEM and TEM images of PLA-based nanofibers electrospun by a,b) CE and c-e) PE with OA-MION concentration of $0.2 \mathrm{mg} \mathrm{mL}^{-1}$. (C) a) Antimicrobial activities of PLA, PE without Van, PE and CE electrospun nanofiber membranes against $S$. aureus and b) tensile stress-strain curves of PLA and PE electrospun nanofiber membranes. Reproduced with permission. ${ }^{47}$ Copyright 2016, Wiley publishers.

HAp-functionalized PCL fibers were prepared by electrospinning HAp-stabilized $\mathrm{O} / \mathrm{W}$ Pickering emulsions ${ }^{75}$. HAp was shown to be uniformly dispersed across the fiber length, and the fibers were found to have significantly higher cell proliferation than pure PCL fibers. HAp was also shown to enhance the storage modulus of fibers by facilitating the interaction between the two phases of the emulsion.

PCL composite fibers were made from a W/O (PCL solution in 70:30 $\mathrm{CH}_{2} \mathrm{Cl}_{2}$ /dimethylformamide (DMF)) Pickering emulsion stabilized by hydrophobically modified $\mathrm{SiO}_{2}\left(\mathrm{~m}-\mathrm{SiO}_{2}\right)^{76}$. In addition to the stabilizer role, $\mathrm{m}-\mathrm{SiO}_{2}$ also acted as a nucleating agent for PCL and a reinforcing agent for the final composite, which was endowed with better tensile properties and suitable cell proliferation.

\subsubsection{Films}

Reinforcement of polymer films with nanoparticles is desirable, yet the effectiveness is often compromised by uneven dispersion ${ }^{77-79}$. Nanoparticles can be uniformly dispersed into a polymer matrix by combining the Pickering emulsion approach with compression molding. The nanoparticles play dual roles as Pickering 
stabilizers and as nanofillers for the final composites.

\section{Polymerization approach}

Highly transparent nanocellulose/polystyrene composite films were prepared by hot-pressing composite microspheres obtained from emulsion polymerization using a surface-carboxylated nanocellulose as the stabilizer (Figure 2.6) ${ }^{80}$. These composite films showed good mechanical properties and thermal dimensional stability and were highly transparent to optical light (transmittance of $88 \%$ at $600 \mathrm{~nm}$ ), a value that is as high as that of PS films (90\%). Appropriate oil can be added into monomers (oil phase) to tune the interfacial tensions between the emulsifiers and polymers ${ }^{81}$.

(A)

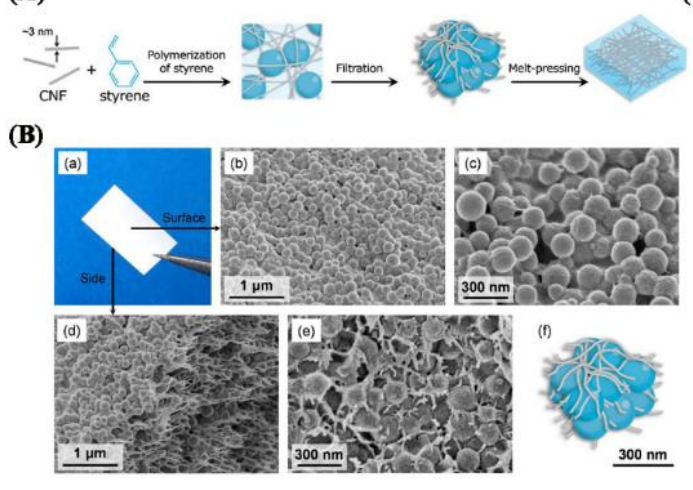

(C)
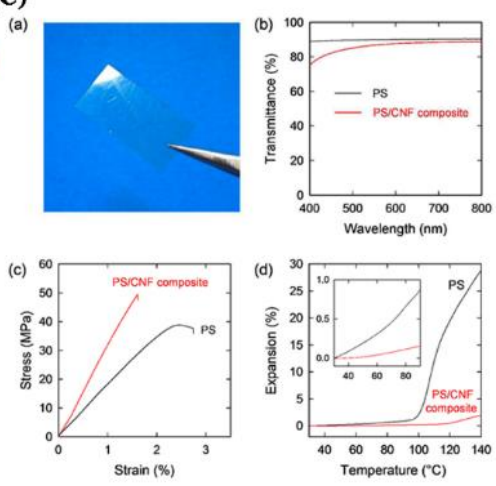

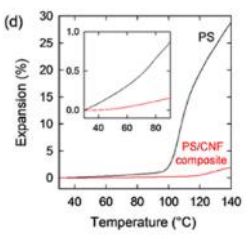

Figure 2.6 (A) The preparation process of transparent nanocellulose/polystyrene composite films from Pickering emulsion polymerization. (B) (a) Photograph, (b-e) SEM images, and (f) schematic illustration of the PS/CNF composite with $12 \% \mathrm{w} / \mathrm{w}$ CNF before melt pressing. (b, c) Film surface, (d) edge, and (e) side surface. (C) (a) Photograph of the PS/CNF composite film with $12 \% \mathrm{w} / \mathrm{w}$ CNF after melt pressing; (b) UV-vis transmittance spectra; (c) stress-strain curves; and (d) thermal expansion behavior of the PS/CNF composite and PS films. Reproduced with permission. ${ }^{80}$ Copyright 2017, American Chemical Society.

A similar method was used to prepare electrically conductive graphene/ polystyrene composite films with graphene as a solid stabilizer ${ }^{82}$. The thermal, mechanical, and conductive properties of polystyrene were remarkably improved with low loading of the functionalized graphene due to good dispersion of the functionalized graphene in the polystyrene matrix. 


\section{Solvent evaporation approach}

In our group, CNF/PLA composites with CNF contents of $10 \%, 20 \%$ and $30 \%$ were prepared by evaporating $\mathrm{CH}_{2} \mathrm{Cl}_{2}$ from the corresponding $\mathrm{O} / \mathrm{W}$ Pickering emulsions ${ }^{29}$. The composites displayed an enhanced storage modulus and flexural performance compared with neat PLA. RC/PLA composites with RC contents of $1 \%$, $1.5 \%$ and $2 \%$ were also prepared via a similar approach ${ }^{31}$. An image of the emulsion and an SEM image of the RC/PLA microspheres are shown in Figure 2.7A. The resulting RC/PLA composites had enhanced crystallization and tensile strength of up to $34 \%$ compared with native PLA, while the transmittance in the visible range remained above $70 \%$.

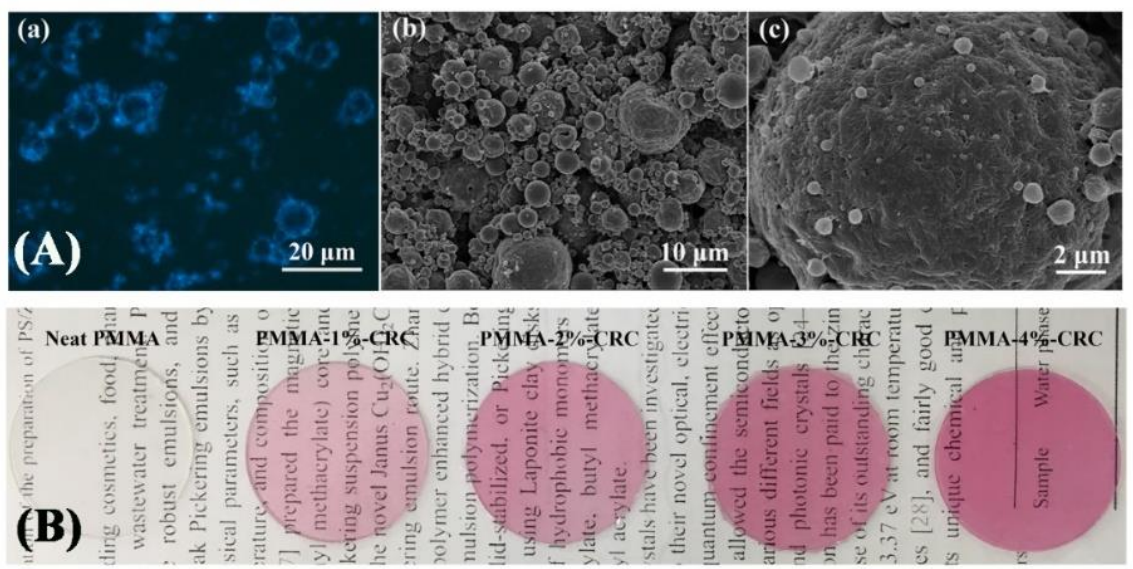

Figure 2.7 (A) (a) Fluorescence micrographs of a Pickering emulsion stabilized by RC; (b, c) FE-SEM image of RC/PLA composites after filtration. Reproduced with permission. ${ }^{31}$ Copyright 2018, Elsevier. (B) Photograph of compressed poly(methyl methacrylate) (PMMA) nanocomposites with different dyed RC (DRC) loadings. Reproduced with permission. ${ }^{83}$ Copyright 2020, Elsevier.

In addition to serving as the reinforcing agent, nanoparticles can also impart polymers with functionality. $\mathrm{Fu}$ and coworkers reported conductive ternary PLA/CNC/carbon nanotube (CNT) composite films prepared from an $\mathrm{O}\left(\mathrm{CH}_{2} \mathrm{Cl}_{2}\right.$ solution of PLA)/W Pickering emulsion ${ }^{84}$. The composite films showed an outstanding EMI SE of $41.8 \mathrm{~dB}$ with only $4.3 \mathrm{wt} . \% \mathrm{CNT}$ loading. In our group, we prepared colored PMMA composites based on Pickering emulsion templates using the reactive red dyed $\mathrm{RC}^{83}$. A photograph of these composite films is shown in Figure 2.7B. 


\subsubsection{Porous matrices}

Porous matrices with well-defined pore structures and high specific surface areas are attracting considerable attention due to their multifarious applications as supports for catalysts, scaffolds for tissue engineering and vehicles for drug delivery ${ }^{85-88}$.

\section{Polymerization approach}

High-interphase Pickering emulsions (HIPEs) are defined as emulsions where the volume of the internal phase is higher than $74 \%$ of the total emulsion ${ }^{89}$. HIPEs are frequently used in the food, pharmaceutical and cosmetic industries. HIPE templating is an effective approach to prepare highly porous polymer composites with potential applications as scaffolds in tissue engineering, sensor materials and separation media ${ }^{90-93}$.

Bismarck and coworkers prepared porous polymers from W/O (styrene/poly(ethylene glycol) dimethacrylate) HIPEs stabilized by OA-modified $\mathrm{SiO}_{2}$ nanoparticles ${ }^{94}$. They also synthesized highly permeable macroporous polymers from W/O (styrene, divinylbenzene) HIPEs with average pore sizes of $100 \mu \mathrm{m}$ and average pore throat sizes of 19-26 $\mu \mathrm{m}$ from HIPEs stabilized by calcium chloride dihydrate $\left(\mathrm{CaCl}_{2} 2 \mathrm{H}_{2} \mathrm{O}\right)$ and a nonionic polymeric surfactant ${ }^{41}$. Compared to their counterparts with open pores synthesized from surfactant-stabilized W/O HIPEs (Figure 2.8A), the porous polymers synthesized from particle-stabilized W/O HIPEs have a closed-cell structure (Figure 2.8B). When small amounts of a nonionic polymeric surfactant were added to such Pickering-HIPE templates, the formation of poly-Pickering-HIPEs with an open porous structure (Figure 2.8C) and greatly enhanced permeability (more than 5-fold) larger than conventional polyHIPEs resulted.
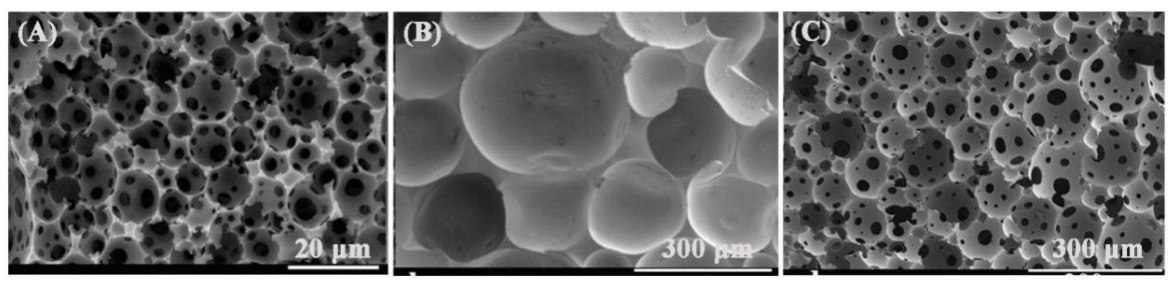

Figure 2.8 SEM images of (A) conventional polyHIPEs synthesized from a surfactantstabilized HIPE template, (B) poly-Pickering-HIPEs synthesized from a Pickering-HIPE template stabilized by only $\mathrm{CaCl}_{2} 2 \mathrm{H}_{2} \mathrm{O}$, and (C) poly-Pickering-HIPEs synthesized from a 
Pickering-HIPE template stabilized by $\mathrm{CaCl}_{2} 2 \mathrm{H}_{2} \mathrm{O}$ and Hypermer 2296 (a nonionic polymeric surfactant). Reproduced with permission. ${ }^{41}$ Copyright 2010, Wiley publishers.

Magnetic microporous polymers were prepared from W/O HIPEs stabilized by OA-modified iron oxide nanoparticles ${ }^{95}$. Depending on the OA content, the nanoparticles were either arranged exclusively at the oil-water interface or partially dispersed into the oil phase. The porous composites showed superparamagnetic behaviors and a relatively high magnetic moment. Similarly, OA-modified $\mathrm{TiO}_{2}$ particles could also be used to stabilize HIPEs to prepare porous polymers ${ }^{96}$.

3D macroporous PS composites were successfully prepared using W/O HIPEs stabilized by GO flakes modified with the cationic surfactant cetyltrimethylammonium bromide ${ }^{97}$. Macroporous polymers with a smaller void size, a narrow size distribution, and a large specific surface area of up to $490 \mathrm{~m}^{2} / \mathrm{g}$ were accessible when a higher concentration of the modified GO was used.

$\mathrm{Hu}$ and coworkers prepared hydrophilic porous polymers from $\mathrm{O} / \mathrm{W}$ (acrylamide and N,N-methylene bisacrylamide) HIPEs stabilized by nanodispersed poly(urethane urea) particles ${ }^{98}$. The morphology of the hydrophilic polymers could be conveniently tailored by changing the $\mathrm{O} / \mathrm{W}$ ratio or concentration of PU particles. Lignin particlestabilized $\mathrm{O} / \mathrm{W}$ HIPEs were applied in the preparation of macroporous melamine formaldehyde polymers ${ }^{99}$. The structure of the resulting polymer composites transformed from a closed cell to an open cell with increasing monomer concentration.

Artemisia argyi oil (AAO)-loaded macroporous antibacterial hydrogels were reported by Wang and his coworker by using O/W HIPE templates that were dually stabilized by hydrophilic $\mathrm{SiO}_{2}$ and the nonionic surfactant Tween $80^{100}$. The resulting hydrogels showed a high compressive stress of $234.6 \mathrm{kPa}$ and good AAO release activity. Similarly, hydrophilic polymers synthesized from acrylamide and N,Nmethylene bisacrylamide with a well-defined open-cell structure could be prepared via HIPE templates stabilized by $\mathrm{TiO}_{2}$ nanoparticles and a small amount of Tween $85^{101}$.

\section{Solvent evaporation approach}

Wang and coworkers prepared HAp/PLLA interconnected microporous composites by evaporating $\mathrm{CH}_{2} \mathrm{Cl}_{2}$ from the W/O (PLLA in $\mathrm{CH}_{2} \mathrm{Cl}_{2}$ ) Pickering emulsion $^{102}$. The pore structure of the composite could be easily tuned by varying the concentration of the g-HAp nanoparticles. When used as scaffolds, the resulting composite showed a sustained release of ibuprofen that was adjustable by changing 
the $\mathrm{pH}$ of the release medium ${ }^{13}$. The scaffold had excellent biodegradable and bioactive properties and could be used in bone tissue engineering applications. Similarly, g-HAp/PCL nanocomposite porous scaffolds could be prepared and used to deliver drugs ${ }^{46}$.

As shown in Figure 2.9, porous matrices can also be fabricated by a combination of $3 \mathrm{D}$ printing and Pickering emulsion templates ${ }^{103}$. In this work, the authors provided a general method to build 3D nanocomposites with the continuous phase of a nanomaterial, which allows for arbitrary hybridization and patterns.

Hierarchical microporous biocompatible (HmPB) scaffolds were prepared by $3 \mathrm{D}$ printing of a W/O (PCL and PLLA in $\mathrm{CH}_{2} \mathrm{Cl}_{2}$ ) HIPE stabilized by hydrophobically modified $\mathrm{SiO}_{2}$ nanoparticles $\left(\mathrm{h}-\mathrm{SiO}_{2}\right)^{104}$. The $\mathrm{HmPB}$ scaffolds can be used as an enrofloxacin-releasing surgical dressing that meets the demand for anti-inflammation in the early period of implantation.

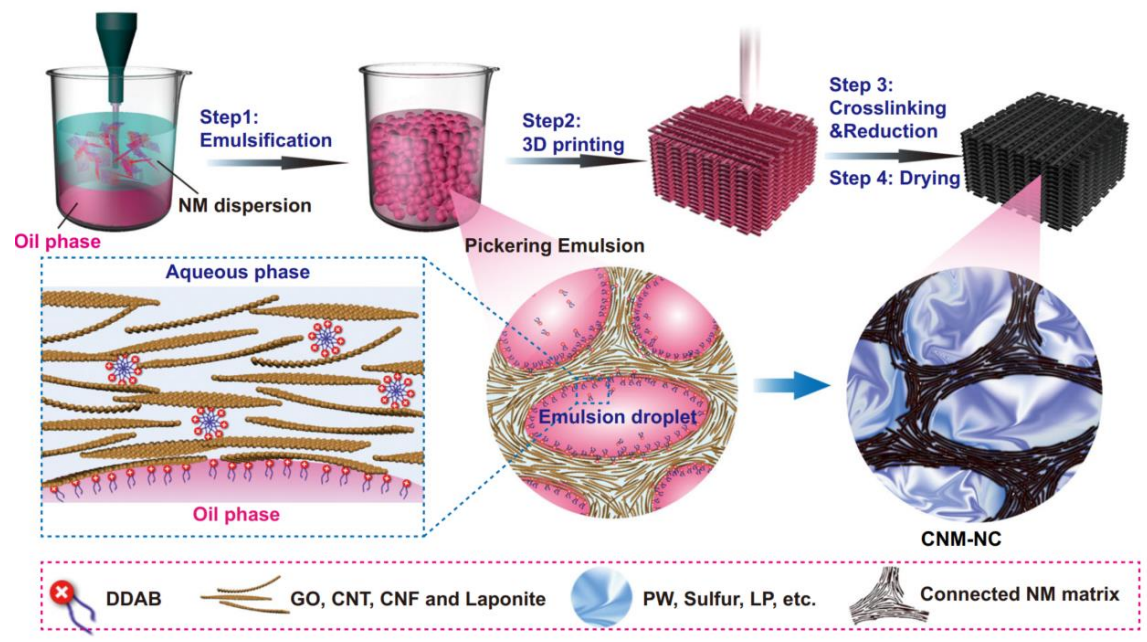

Figure 2.9 Schematic of the 3D printing of a Pickering emulgel. Reproduced with permission. ${ }^{103}$ Copyright 2021, Nature.

\subsection{Conclusions}

Recent advances in the preparation of hybrid polymeric materials via the Pickering emulsion platform from a morphological perspective were summarized. Polymerization and solvent evaporation lead to the formation of a supportive polymer phase after removal of the Pickering emulsion template. Hybrid materials in the forms 
of microspheres, fibers, films, and porous matrices can be fabricated based on the Pickering emulsion platform. Microspheres and microcapsules are usually prepared by polymerization or solvent evaporation of the discontinuous phase in Pickering emulsions. Functional substances can be encapsulated into microcapsules, which can be used in drug delivery, catalysis, phase change materials and so on. Core-shell fibers are often obtained by electrospinning of a Pickering emulsion where the polymer solution is the oily phase. These fibers could be used for wound dressings, delivery of drugs and bone tissue engineering due to their low tendency for leaching out and low toxicity. Polymer composite films in which nanoparticles are uniformly dispersed can be prepared by compression of microspheres from Pickering emulsions. This approach can be used in polymer reinforcement. Porous matrices are usually fabricated by polymerization or solvent evaporation of the continuous phase in HIPEs. These matrices can be used in multifarious applications, including supports for catalysts, scaffolds for tissue engineering, vehicles for drug delivery and the $3 \mathrm{D}$ cell industry. Pickering emulsion platforms are a promising way to fabricate advanced hybrid materials for various applications. 


\section{References}

[1] Faustini, M.; Nicole, L.; Ruiz-Hitzky, E.; Sanchez, C. History of organicinorganic hybrid materials: prehistory, art, science, and advanced applications. Advanced Functional Materials 2018, 28 (27), 1704158.

[2] Darder, M.; Aranda, P.; Ruiz-Hitzky, E. Bionanocomposites: a new concept of ecological, bioinspired, and functional hybrid materials. Advanced Materials 2007, 19 (10), 1309-1319.

[3] Wu, J.; Ma, G. H. Recent studies of Pickering emulsions: particles make the difference. Small 2016, 12 (34), 4633-4648.

[4] Piao, S. H.; Gao, C. Y.; Choi, H. J. Pickering emulsion-polymerized conducting polymer nanocomposites and their applications. Chemical Papers 2017, 71 (2), 179-188.

[5] Zhao, S.; Wang, Z.; Kang, H.; Zhang, W.; Li, J.; Zhang, S.; Li, L.; Huang, A. Construction of bioinspired organic-inorganic hybrid composite by celluloseinduced interfacial gelation assisted with Pickering emulsion template. Chemical Engineering Journal 2019, 359, 275-284.

[6] Binks, B. P. Particles as surfactants-similarities and differences. Current Opinion in Colloid \& Interface Science 2002, 7 (1-2), 21-41.

[7] Chevalier, Y.; Bolzinger, M.-A. Emulsions stabilized with solid nanoparticles: Pickering emulsions. Colloids and Surfaces A: Physicochemical and Engineering Aspects 2013, 439, 23-34.

[8] Jiménez Saelices, C.; Capron, I. Design of Pickering micro-and nanoemulsions based on the structural characteristics of nanocelluloses. Biomacromolecules 2018, 19 (2), 460-469.

[9] Tambe, D. E.; Sharma, M. M. Factors controlling the stability of colloidstabilized emulsions: I. An experimental investigation. Journal of Colloid and Interface Science 1993, 157 (1), 244-253.

[10] Binks, B.; Lumsdon, S. Pickering emulsions stabilized by monodisperse latex particles: effects of particle size. Langmuir 2001, 17 (15), 4540-4547.

[11] Jiang, Y.; Liu, L.; Wang, B.; Yang, X.; Chen, Z.; Zhong, Y.; Zhang, L.; Mao, Z.; $\mathrm{Xu}, \mathrm{H}$;; Sui, X. Polysaccharide-based edible emulsion gel stabilized by regenerated cellulose. Food Hydrocolloids 2019, 91, 232-237.

[12] Jiang, Y.; Liu, L.; Wang, B.; Sui, X.; Zhong, Y.; Zhang, L.; Mao, Z.; Xu, H. 
Cellulose-rich oleogels prepared with an emulsion-templated approach. Food Hydrocolloids 2018, 77, 460-464.

[13] Hu, Y.; Zou, S.; Chen, W.; Tong, Z.; Wang, C. Mineralization and drug release of hydroxyapatite/poly (l-lactic acid) nanocomposite scaffolds prepared by Pickering emulsion templating. Colloids and Surfaces B: Biointerfaces 2014, $122,559-565$.

[14] Zhou, J.; Qiao, X.; Binks, B. P.; Sun, K.; Bai, M.; Li, Y.; Liu, Y. Magnetic Pickering emulsions stabilized by $\mathrm{Fe}_{3} \mathrm{O}_{4}$ nanoparticles. Langmuir 2011, 27 (7), 3308-3316.

[15] Binks, B. P.; Lumsdon, S. Influence of particle wettability on the type and stability of surfactant-free emulsions. Langmuir 2000, 16 (23), 8622-8631.

[16] Frelichowska, J.; Bolzinger, M.-A.; Pelletier, J.; Valour, J.-P.; Chevalier, Y. Topical delivery of lipophilic drugs from o/w Pickering emulsions. International Journal of Pharmaceutics 2009, 371 (1-2), 56-63.

[17] Zhao, X.; Yu, G.; Li, J.; Feng, Y.; Zhang, L.; Peng, Y.; Tang, Y.; Wang, L. Ecofriendly pickering emulsion stabilized by silica nanoparticles dispersed with high-molecular-weight amphiphilic alginate derivatives. ACS Sustainable Chemistry \& Engineering 2018, 6 (3), 4105-4114.

[18] Yüce, E.; Krajnc, P.; Mert, H.; Mert, E. Influence of nanoparticles and antioxidants on mechanical properties of titania/polydicyclopentadiene polyHIPEs: A statistical approach. Journal of Applied Polymer Science 2019, $136(7), 46913$.

[19] Han, S.; Choi, J.; Seo, Y. P.; Park, I. J.; Choi, H. J.; Seo, Y. High-performance magnetorheological suspensions of pickering-emulsion-polymerized polystyrene $/ \mathrm{Fe}_{3} \mathrm{O}_{4}$ particles with enhanced stability. Langmuir 2018, 34 (8), 2807-2814.

[20] Costa, A. L. R.; Gomes, A.; Tibolla, H.; Menegalli, F. C.; Cunha, R. L. Cellulose nanofibers from banana peels as a Pickering emulsifier: High-energy emulsification processes. Carbohydrate Polymers 2018, 194, 122-131.

[21] Fadil, Y.; Jasinski, F.; Guok, T. W.; Thickett, S. C.; Minami, H.; Zetterlund, P. B. Pickering miniemulsion polymerization using graphene oxide: effect of addition of a conventional surfactant. Polymer Chemistry 2018, 9 (24), 3368-3378.

[22] Jiao, B.; Shi, A.; Wang, Q.; Binks, B. P. High-internal-phase Pickering emulsions 
stabilized solely by peanut-protein-isolate microgel particles with multiple potential applications. Angewandte Chemie 2018, 130 (30), 9418-9422.

[23] Zhu, H.; Lei, L.; Li, B.-G.; Zhu, S. Development of novel materials from polymerization of Pickering emulsion templates. Polymer Reaction Engineering of Dispersed Systems 2017, 101-119.

[24] Kimmins, S. D.; Cameron, N. R. Functional porous polymers by emulsion templating: Recent advances. Advanced Functional Materials 2011, 21 (2), 211 225.

[25] Silverstein, M. S. PolyHIPEs: Recent advances in emulsion-templated porous polymers. Progress in Polymer Science 2014, 39 (1), 199-234.

[26] Brun, N.; Ungureanu, S.; Deleuze, H.; Backov, R. Hybrid foams, colloids and beyond: From design to applications. Chemical Society Reviews 2011, 40 (2), 771-788.

[27] Schrade, A.; Landfester, K.; Ziener, U. Pickering-type stabilized nanoparticles by heterophase polymerization. Chemical Society Reviews 2013, 42 (16), 68236839.

[28] Dickinson, E. Biopolymer-based particles as stabilizing agents for emulsions and foams. Food Hydrocolloids 2017, 68, 219-231.

[29] Zhang, Y.; Wu, J.; Wang, B.; Sui, X.; Zhong, Y.; Zhang, L.; Mao, Z.; Xu, H. Cellulose nanofibril-reinforced biodegradable polymer composites obtained via a Pickering emulsion approach. Cellulose 2017, 24 (8), 3313-3322.

[30] Zhang, Y.; Cui, L.; Xu, H.; Feng, X.; Wang, B.; Pukánszky, B.; Mao, Z.; Sui, X. Poly (lactic acid)/cellulose nanocrystal composites via the Pickering emulsion approach: Rheological, thermal and mechanical properties. International Journal of Biological Macromolecules 2019, 137, 197-204.

[31] Zhang, Y.; Jiang, Y.; Han, L.; Wang, B.; Xu, H.; Zhong, Y.; Zhang, L.; Mao, Z.; Sui, X. Biodegradable regenerated cellulose-dispersed composites with improved properties via a Pickering emulsion process. Carbohydrate Polymers 2018, 179, 86-92.

[32] Li, X.; Hegyesi, N.; Zhang, Y.; Mao, Z.; Feng, X.; Wang, B.; Pukánszky, B.; Sui, $\mathrm{X}$. Poly (lactic acid)/lignin blends prepared with the Pickering emulsion template method. European Polymer Journal 2019, 110, 378-384.

[33] Munyazesa, F. X.; Zhang, Y.; Wang, B.; Wang, C.; Feng, X.; Mao, Z.; Chen, Y.; 
Sui, X. Pickering emulsion process assisted construction of regenerated chitin reinforced poly (lactic acid) blends. International Journal of Biological Macromolecules 2019, 140, 10-16.

[34] Jiang, Y.; Zhang, Y.; Ding, L.; Joshua, A.; Wang, B.; Feng, X.; Chen, Z.; Mao, Z.; Sui, X. Regenerated cellulose-dispersed polystyrene composites enabled via Pickering emulsion polymerization. Carbohydrate Polymers 2019, 223, 115079.

[35] Li, X.; Wang, Y.; Wang, B.; Feng, X.; Mao, Z.; Sui, X. Antibacterial phase change microcapsules obtained with lignin as the Pickering stabilizer and the reducing agent for silver. International Journal of Biological Macromolecules 2020, 144 , 624-631.

[36] Wang, Y.; Li, X.; Shen, C.; Mao, Z.; Xu, H.; Zhong, Y.; Sui, X.; Feng, X.; Wang, B. Lignin assisted Pickering emulsion polymerization to microencapsulate 1tetradecanol for thermal management. International Journal of Biological Macromolecules 2020, 146, 1-8.

[37] Lotierzo, A.; Bon, S. A. A mechanistic investigation of Pickering emulsion polymerization. Polymer Chemistry 2017, 8 (34), 5100-5111.

[38] Cauvin, S.; Colver, P. J.; Bon, S. A. Pickering stabilized miniemulsion polymerization: preparation of clay armored latexes. Macromolecules 2005, 38 (19), 7887-7889.

[39] Gao, Q.; Wang, C.; Liu, H.; Wang, C.; Liu, X.; Tong, Z. Suspension polymerization based on inverse Pickering emulsion droplets for thermosensitive hybrid microcapsules with tunable supracolloidal structures. Polymer 2009, 50 (12), 2587-2594.

[40] Kim, S. H.; Kim, J. H.; Choi, H. J.; Park, J. Pickering emulsion polymerized poly (3, 4-ethylenedioxythiophene): poly (styrenesulfonate)/polystyrene composite particles and their electric stimuli-response. RSC Advances 2015, 5 (88), 72387 72393.

[41] Ikem, V. O.; Menner, A.; Horozov, T. S.; Bismarck, A. Highly permeable macroporous polymers synthesized from pickering medium and high internal phase emulsion templates. Advanced Materials 2010, 22 (32), 3588-3592.

[42] Zhao, Z.; Li, C.; Dong, Z.; Yang, Y.; Zhang, L.; Zhuo, S.; Zhou, X.; Xu, Y.; Jiang, L.; Liu, M. Adaptive superamphiphilic organohydrogels with reconfigurable surface topography for programming unidirectional liquid transport. Advanced 
Functional Materials 2019, 29 (16), 1807858.

[43] Zhao, Z.; Zhang, K.; Liu, Y.; Zhou, J.; Liu, M. Highly stretchable, shape memory organohydrogels using phase-transition microinclusions. Advanced Materials 2017, 29 (33), 1701695.

[44] Zhao, Z.; Zhuo, S.; Fang, R.; Zhang, L.; Zhou, X.; Xu, Y.; Zhang, J.; Dong, Z.; Jiang, L.; Liu, M. Dual-programmable shape-morphing and self-healing organohydrogels through orthogonal supramolecular heteronetworks. Advanced Materials 2018, 30 (51), 1804435.

[45] Liu, X.; Okada, M.; Maeda, H.; Fujii, S.; Furuzono, T. Hydroxyapatite/biodegradable poly (1-lactide-co-e-caprolactone) composite microparticles as injectable scaffolds by a Pickering emulsion route. Acta Biomaterialia 2011, 7 (2), 821-828.

[46] Hu, Y.; Gao, H.; Du, Z.; Liu, Y.; Yang, Y.; Wang, C. Pickering high internal phase emulsion-based hydroxyapatite-poly ( $\varepsilon$-caprolactone) nanocomposite scaffolds. Journal of Materials Chemistry B 2015, 3 (18), 3848-3857.

[47] Cai, N.; Han, C.; Luo, X.; Chen, G.; Dai, Q.; Yu, F. Fabrication of core/shell nanofibers with desirable mechanical and antibacterial properties by Pickering emulsion electrospinning. Macromolecular Materials and Engineering 2017, 302 (3), 1600364.

[48] Gao, D.; Chang, R.; Lyu, B.; Ma, J.; Duan, X. Preparation of epoxy-acrylate copolymer/nano-silica via Pickering emulsion polymerization and its application as printing binder. Applied Surface Science 2018, 435, 195-202.

[49] Luo, Q.; Wang, Y.; Chen, Z.; Wei, P.; Yoo, E.; Pentzer, E. Pickering emulsiontemplated encapsulation of ionic liquids for contaminant removal. ACS Applied Materials \& Interfaces 2019, 11 (9), 9612-9620.

[50] Wang, H.; Zhao, L.; Song, G.; Tang, G.; Shi, X. Organic-inorganic hybrid shell microencapsulated phase change materials prepared from $\mathrm{SiO}_{2} /$ TiC-stabilized Pickering emulsion polymerization. Solar Energy Materials and Solar Cells 2018, 175, 102-110.

[51] Zhou, T.; Zhang, K.; Kamra, T.; Bülow, L.; Ye, L. Preparation of protein imprinted polymer beads by Pickering emulsion polymerization. Journal of Materials Chemistry B 2015, 3 (7), 1254-1260.

[52] Chen, T.; Colver, P. J.; Bon, S. A. Organic-inorganic hybrid hollow spheres 
prepared from $\mathrm{TiO}_{2}$-stabilized Pickering emulsion polymerization. Advanced Materials 2007, 19 (17), 2286-2289.

[53] Dao, T. D.; Erdenedelger, G.; Jeong, H. M. Water-dispersible graphene designed as a Pickering stabilizer for the suspension polymerization of poly (methyl methacrylate)/graphene core-shell microsphere exhibiting ultra-low percolation threshold of electrical conductivity. Polymer 2014, 55 (18), 4709-4719.

[54] Jeng, J.; Chen, T.-Y.; Lee, C.-F.; Liang, N.-Y.; Chiu, W.-Y. Growth mechanism and $\mathrm{pH}$-regulation characteristics of composite latex particles prepared from Pickering emulsion polymerization of aniline/ZnO using different hydrophilicities of oil phases. Polymer 2008, 49 (15), 3265-3271.

[55] Chae, H. S.; Piao, S. H.; Han, W. J.; Choi, H. J. Core/shell polystyrene/magnetite hybrid nanoparticles fabricated by Pickering emulsion polymerization and their magnetorheological response. Macromolecular Chemistry and Physics 2018, $219(5), 1700408$.

[56] Colver, P. J.; Colard, C. A.; Bon, S. A. Multilayered nanocomposite polymer colloids using emulsion polymerization stabilized by solid particles. Journal of the American Chemical Society 2008, 130 (50), 16850-16851.

[57] Nypelö, T.; Rodriguez-Abreu, C.; Kolen'ko, Y. V.; Rivas, J.; Rojas, O. J. Microbeads and hollow microcapsules obtained by self-assembly of Pickering magneto-responsive cellulose nanocrystals. ACS Applied Materials \& Interfaces 2014, 6 (19), 16851-16858.

[58] Chen, K.; Zhou, J.; Hu, J.; Zhang, J.; Heng, T.; Xu, C.; Wang, X.; Liu, J.; Yu, K. Preparation of $\mathrm{pH}$-responsive dual-compartmental microcapsules via Pickering emulsion and their application in multifunctional textiles. ACS Applied Materials \& Interfaces 2020, 13 (1), 1234-1244.

[59] Zhang, K.; Wu, W.; Guo, K.; Chen, J.-F.; Zhang, P.-Y. Magnetic polymer enhanced hybrid capsules prepared from a novel Pickering emulsion polymerization and their application in controlled drug release. Colloids and Surfaces A: Physicochemical and Engineering Aspects 2009, 349 (1-3), 110-116.

[60] Yi, H.; Yang, Y.; Gu, X.; Huang, J.; Wang, C. Multilayer composite microcapsules synthesized by Pickering emulsion templates and their application in self-healing coating. Journal of Materials Chemistry A 2015, 3 (26), 1374913757. 
[61] Luo, J.; Chen, Y.; Zheng, Y.; Wang, C.; Wei, W.; Liu, X. Hollow graphenepolyaniline hybrid spheres using sulfonated graphene as Pickering stabilizer for high performance supercapacitors. Electrochimica Acta 2018, 272, 221-232.

[62] Kim, J. B.; Lee, S. Y.; Min, N. G.; Lee, S. Y.; Kim, S. H. Plasmonic janus microspheres created from Pickering emulsion drops. Advanced Materials 2020, 32 (26), 2001384.

[63] Bai, X.; Liu, J.; Xu, Y.; Ma, Y.; Liu, Z.; Pan, J. CO $\mathrm{CO}_{2}$ Pickering emulsion in water templated hollow porous sorbents for fast and highly selective uranium extraction. Chemical Engineering Journal 2020, 387, 124096.

[64] Zou, S.; Liu, H.; Yang, Y.; Wei, Z.; Wang, C. Multihollow nanocomposite microspheres with tunable pore structures by templating Pickering double emulsions. Reactive and Functional Polymers 2013, 73 (9), 1231-1241.

[65] Wang, Z.; Qiu, T.; Guo, L.; Ye, J.; He, L.; Li, X. Polymerization induced shaping of Pickering emulsion droplets: From simple hollow microspheres to molecularly imprinted multicore microrattles. Chemical Engineering Journal 2018, 332, 409-418.

[66] Fujii, S.; Okada, M.; Nishimura, T.; Maeda, H.; Sugimoto, T.; Hamasaki, H.; Furuzono, T.; Nakamura, Y. Hydroxyapatite-armored poly ( $\varepsilon$-caprolactone) microspheres and hydroxyapatite microcapsules fabricated via a Pickering emulsion route. Journal of Colloid and Interface Science 2012, 374 (1), 1-8.

[67] Maeda, H.; Okada, M.; Fujii, S.; Nakamura, Y.; Furuzono, T. Pickering-type water-in-oil-in-water multiple emulsions toward multihollow nanocomposite microspheres. Langmuir 2010, 26 (17), 13727-13731.

[68] Wei, Z.; Wang, C.; Zou, S.; Liu, H.; Tong, Z. $\mathrm{Fe}_{2} \mathrm{O}_{3}$ nanoparticles as particulate emulsifier: Preparation of magnetic and biocompatible PLGA microcapsules. Colloids and Surfaces A: Physicochemical and Engineering Aspects 2011, 392 (1), 116-123.

[69] Wang, F.; Zhang, Y.; Li, X.; Wang, B.; Feng, X.; Xu, H.; Mao, Z.; Sui, X. Cellulose nanocrystals-composited poly (methyl methacrylate) encapsulated neicosane via a Pickering emulsion-templating approach for energy storage. Carbohydrate Polymers 2020, 234, 115934.

[70] Li, X.; Su, Y.; Liu, S.; Tan, L.; Mo, X.; Ramakrishna, S. Encapsulation of proteins in poly (1-lactide-co-caprolactone) fibers by emulsion electrospinning. Colloids 
and Surfaces B: Biointerfaces 2010, 75 (2), 418-424.

[71] Goh, Y.-F.; Shakir, I.; Hussain, R. Electrospun fibers for tissue engineering, drug delivery, and wound dressing. Journal of Materials Science 2013, 48 (8), 3027 3054.

[72] Hu, X.; Liu, S.; Zhou, G.; Huang, Y.; Xie, Z.; Jing, X. Electrospinning of polymeric nanofibers for drug delivery applications. Journal of Controlled Release 2014, 185, 12-21.

[73] Yoshimoto, H.; Shin, Y.; Terai, H.; Vacanti, J. A biodegradable nanofiber scaffold by electrospinning and its potential for bone tissue engineering. Biomaterials 2003, 24 (12), 2077-2082.

[74] Dai, Y.; Niu, J.; Liu, J.; Yin, L.; Xu, J. In situ encapsulation of laccase in microfibers by emulsion electrospinning: Preparation, characterization, and application. Bioresource Technology 2010, 101 (23), 8942-8947.

[75] Samanta, A.; Takkar, S.; Kulshreshtha, R.; Nandan, B.; Srivastava, R. K. Hydroxyapatite stabilized pickering emulsions of poly ( $\varepsilon$-caprolactone) and their composite electrospun scaffolds. Colloids and Surfaces A: Physicochemical and Engineering Aspects 2017, 533, 224-230.

[76] Samanta, A.; Takkar, S.; Kulshreshtha, R.; Nandan, B.; Srivastava, R. K. Facile fabrication of composite electrospun Nanofibrous matrices of poly ( $\varepsilon$ caprolactone)-silica based Pickering emulsion. Langmuir 2017, 33 (32), 8062 8069.

[77] Almasi, H.; Ghanbarzadeh, B.; Dehghannya, J.; Entezami, A. A.; Asl, A. K. Novel nanocomposites based on fatty acid modified cellulose nanofibers/poly (lactic acid): Morphological and physical properties. Food Packaging and Shelf Life 2015, 5, 21-31.

[78] Lee, K.-Y.; Aitomäki, Y.; Berglund, L. A.; Oksman, K.; Bismarck, A. On the use of nanocellulose as reinforcement in polymer matrix composites. Composites Science and Technology 2014, 105, 15-27.

[79] Mariano, M.; El Kissi, N.; Dufresne, A. Cellulose nanocrystals and related nanocomposites: Review of some properties and challenges. Journal of Polymer Science Part B: Polymer Physics 2014, 52 (12), 791-806.

[80] Fujisawa, S.; Togawa, E.; Kuroda, K. Facile route to transparent, strong, and thermally stable nanocellulose/polymer nanocomposites from an aqueous 
Pickering emulsion. Biomacromolecules 2017, 18 (1), 266-271.

[81] Kim, D. W.; Shin, J.; Choi, S. Q. Nano-dispersed cellulose nanofibrils-PMMA composite from Pickering emulsion with tunable interfacial tensions. Carbohydrate Polymers 2020, 247, 116762.

[82] Zhang, L.; Shi, T.; Wu, S.; Zhou, H. Graphene/polystyrene nanocomposites synthesized via Pickering emulsion polymerization. High Performance Polymers 2014, 26 (2), 156-165.

[83] Zhang, Y.; Ding, L.; Ming, B.; Wang, B.; Feng, X.; Vancso, G. J.; Sui, X. Making polymers colored and stiffer by dyed regenerated cellulose employing Pickering emulsions. Colloids and Surfaces A: Physicochemical and Engineering Aspects 2020, 592, 124601.

[84] Yu, B.; Zhao, Z.; Fu, S.; Meng, L.; Liu, Y.; Chen, F.; Wang, K.; Fu, Q. Fabrication of PLA/CNC/CNT conductive composites for high electromagnetic interference shielding based on Pickering emulsions method. Composites Part A: Applied Science and Manufacturing 2019, 125, 105558.

[85] Huo, J.; Marcello, M.; Garai, A.; Bradshaw, D. MOF-polymer composite microcapsules derived from Pickering emulsions. Advanced Materials 2013, 25 (19), 2717-2722.

[86] Blaker, J. J.; Lee, K.-Y.; Li, X.; Menner, A.; Bismarck, A. Renewable nanocomposite polymer foams synthesized from Pickering emulsion templates. Green Chemistry 2009, 11 (9), 1321-1326.

[87] Menner, A.; Ikem, V.; Salgueiro, M.; Shaffer, M. S.; Bismarck, A. High internal phase emulsion templates solely stabilised by functionalised titania nanoparticles. Chemical Communications 2007, (41), 4274-4276.

[88] Stocco, A.; Rio, E.; Binks, B. P.; Langevin, D. Aqueous foams stabilized solely by particles. Soft Matter 2011, 7 (4), 1260-1267.

[89] Capron, I.; Cathala, B. Surfactant-free high internal phase emulsions stabilized by cellulose nanocrystals. Biomacromolecules 2013, 14 (2), 291-296.

[90] Bokhari, M.; Carnachan, R. J.; Przyborski, S. A.; Cameron, N. R. Emulsiontemplated porous polymers as scaffolds for three dimensional cell culture: effect of synthesis parameters on scaffold formation and homogeneity. Journal of Materials Chemistry 2007, 17 (38), 4088-4094.

[91] Zhao, C.; Danish, E.; Cameron, N. R.; Kataky, R. Emulsion-templated porous 
materials (PolyHIPEs) for selective ion and molecular recognition and transport: applications in electrochemical sensing. Journal of Materials Chemistry 2007, 17 (23), 2446-2453.

[92] Krajnc, P.; Leber, N.; Štefanec, D.; Kontrec, S.; Podgornik, A. Preparation and characterisation of poly (high internal phase emulsion) methacrylate monoliths and their application as separation media. Journal of Chromatography A 2005, 1065 (1), 69-73.

[93] Guan, X.; Jiang, H.; Ngai, T. Pickering high internal phase emulsions templated super-hydrophobic-oleophilic elastic foams for highly efficient oil/water separation. ACS Applied Polymer Materials 2020, 2 (12), 5664-5673.

[94] Ikem, V. O.; Menner, A.; Bismarck, A. High internal phase emulsions stabilized solely by functionalized silica particles. Angewandte Chemie 2008, 120 (43), 8401-8403.

[95] Vílchez, A.; Rodríguez-Abreu, C.; Esquena, J.; Menner, A.; Bismarck, A. Macroporous polymers obtained in highly concentrated emulsions stabilized solely with magnetic nanoparticles. Langmuir 2011, 27 (21), 13342-13352.

[96] Ikem, V. O.; Menner, A.; Bismarck, A. High-porosity macroporous polymers sythesized from titania-particle-stabilized medium and high internal phase emulsions. Langmuir 2010, 26 (11), 8836-8841.

[97] Zheng, Z.; Zheng, X.; Wang, H.; Du, Q. Macroporous graphene oxide-polymer composite prepared through Pickering high internal phase emulsions. ACS Applied Materials \& Interfaces 2013, 5 (16), 7974-7982.

[98] Zhu, Y.; Zhang, S.; Hua, Y.; Chen, J.; Hu, C. P. Hydrophilic porous polymers based on high internal phase emulsions solely stabilized by poly (urethane urea) nanoparticles. Polymer 2010, 51 (16), 3612-3617.

[99] Yang, Y.; Wei, Z.; Wang, C.; Tong, Z. Lignin-based Pickering HIPEs for macroporous foams and their enhanced adsorption of copper (II) ions. Chemical Communications 2013, 49 (64), 7144-7146.

[100] Zou, S.; Wei, Z.; Hu, Y.; Deng, Y.; Tong, Z.; Wang, C. Macroporous antibacterial hydrogels with tunable pore structures fabricated by using Pickering high internal phase emulsions as templates. Polymer Chemistry 2014, 5 (14), 4227 4234.

[101] Hua, Y.; Zhang, S.; Zhu, Y.; Chu, Y.; Chen, J. Hydrophilic polymer foams with 
well-defined open-cell structure prepared from pickering high internal phase emulsions. Journal of Polymer Science Part A: Polymer Chemistry 2013, 51 (10), 2181-2187.

[102] Hu, Y.; Gu, X.; Chen, W.; Zou, S.; Wang, C. Macroporous nanocomposite materials prepared by solvent evaporation from pickering emulsion templates. Macromolecular Materials and Engineering 2014, 299 (9), 1070-1080.

[103] Zhang, Y.; Zhu, G.; Dong, B.; Wang, F.; Tang, J.; Stadler, F. J.; Yang, G.; Hong, S.; Xing, F. Interfacial jamming reinforced Pickering emulgel for arbitrary architected nanocomposite with connected nanomaterial matrix. Nature Communications 2021, 12 (1), 1-9.

[104] Yang, T.; Hu, Y.; Wang, C.; Binks, B. P. Fabrication of hierarchical macroporous biocompatible scaffolds by combining Pickering high internal phase emulsion templates with three-dimensional printing. ACS Applied Materials \& Interfaces 2017, 9 (27), 22950-22958. 


\section{Chapter}

\section{Poly (lactic acid)/Cellulose}

Nanocrystal Composites via the Pickering Emulsion Approach: Rheological, Thermal and Mechanical Properties

The use of nanocellulose to enhance biodegradable polymers is an attractive method, but its effects are often affected by uneven dispersion. Cellulose nanocrystals (CNCs) were evenly dispersed into poly (lactic acid) (PLA) via the Pickering emulsion approach. The PLA/CNC composites prepared were studied by rheological, thermal as well as mechanical measurements. Changes in the rheological characteristics of the composites showed that $\mathrm{CNC}$ promoted the transition of the composites from fluid to solid-like behavior at high temperatures. The introduction of 5 wt.\% CNC improved the crystallinity of PLA considerably and increased the onset temperature of crystallization by about $10{ }^{\circ} \mathrm{C}$. The storage modulus of the composites increased throughout the entire temperature range of testing. Flexural modulus was 
improved considerably. All the results indicated that the Pickering emulsion approach improves the dispersion of $\mathrm{CNC}$ in the PLA matrix and $\mathrm{CNC}$ improves efficiently most properties of PLA.

*The contents of this chapter have been published as: Yunchong Zhang, Lu Cui, Hong Xu, Xueling Feng, Bijia Wang, Béla Pukánszky, Zhiping Mao and Xiaofeng Sui; Poly (lactic acid)/Cellulose Nanocrystal Composites via the Pickering Emulsion Approach: Rheological, Thermal and Mechanical Properties; International Journal of Biological Macromolecules, 2019, 137, 197-204. 


\subsection{Introduction}

Poly (lactic acid) (PLA) is a biodegradable thermoplastic polyester derived from renewable resources such as corn, wheat or potato. PLA has been extensively studied over the last several decades due to its easy processability, biodegradability and biocompatibility ${ }^{1-5}$. However, the application of PLA is limited because of its brittleness caused by fast physical ageing, limited thermal stability and slow rate of crystallization ${ }^{6}$.

Cellulose nanocrystal (CNC), one of the most promising reinforcements for nanocomposites, has attracted considerable attention because it is easily available, relatively cheap, nontoxic, has nanoscale dimensions, impressive mechanical properties, low density, and it is also biodegradable and biocompatible ${ }^{7-12}$. The typical size of the crystal ranges from 5 to $15 \mathrm{~nm}$ in width and from 100 to $500 \mathrm{~nm}$ in length ${ }^{13-}$ ${ }^{15}$. Recently, Kontturi and coworkers ${ }^{16}$ reported an effective approach using hydrogen chloride vapor to prepare CNC. Nanocrystals produced by the hydrogen chloride vapor approach do not have electrostatic charge, and the yield of the process can reach $90 \%$. Currently, two major techniques are used for the preparation of CNCs nanocomposites. The first technique is solvent casting through the evaporation of water or an organic solvent. Huang and coworkers ${ }^{17}$ prepared PLA/acetylated CNC composites by solvent casting. The tensile strength of the composites decreased significantly above $6 \%$ acetylated $\mathrm{CNC}$ filler content, because of bad dispersion. The second approach is melt mixing, in which the matrix is extruded in the presence of the desired amount of freeze-dried cellulose nanoparticles. Goffin and coworkers ${ }^{18}$ grafted PLA chains onto the surface of CNC and added the modified CNC to PLA to obtain composites by the melt mixing approach. Although both modified CNCs were readily incorporated into PLA through these two techniques, their reinforcing effect was compromised by the decreased interactions caused by the smaller number of $\mathrm{H}$ bonds resulting from modification.

As reported in our previous publications ${ }^{19-21}$, we have developed an approach using the Pickering emulsion approach for the preparation of PLA composites reinforced with nanocellulose crystals. A Pickering emulsion stabilized with nanocellulose may result in a more homogeneous dispersion of the nanocellulose in the final material than achieved by other techniques. In this communication, we present the use of the Pickering emulsion approach for the preparation of PLA/CNC composites. The first step is the preparation of a Pickering emulsion stabilized by CNC. The second step is the formation of PLA/CNC composite microspheres by the 
removal of $\mathrm{CH}_{2} \mathrm{Cl}_{2}$ and water. The last step is the preparation of the PLA/CNC composites by compression molding of plates from the microspheres. CNCs derived by the hydrogen chloride vapor approach were shown to stabilize efficiently oil-inwater emulsions. The homogeneity of the PLA/CNC composites and their properties were characterized by rheological, thermal and mechanical measurements.

\subsection{Results and Discussion}

The schematic illustration was showed in Figure 3.1. Firstly, PLA was dissolved into $\mathrm{CH}_{2} \mathrm{Cl}_{2}$ forming a homogeneous solution as oil phase. Secondly, CNC was used to stabilize oil-in-water emulsions. Thirdly, PLA/CNC composite microspheres were obtained by slowly evaporation of $\mathrm{CH}_{2} \mathrm{Cl}_{2}$ from the $\mathrm{CNC}$ stabilized Pickering emulsion. Lastly, the even PLA/CNC composites formed by hot-pressing the mixture at $180{ }^{\circ} \mathrm{C}$. In this process, the CNCs were uniformly dispersed into the matrix following the microspheres prepared by Pickering emulsion approach. So, compared to conventional approaches, the PLA/CNC composites have better CNC dispersion.

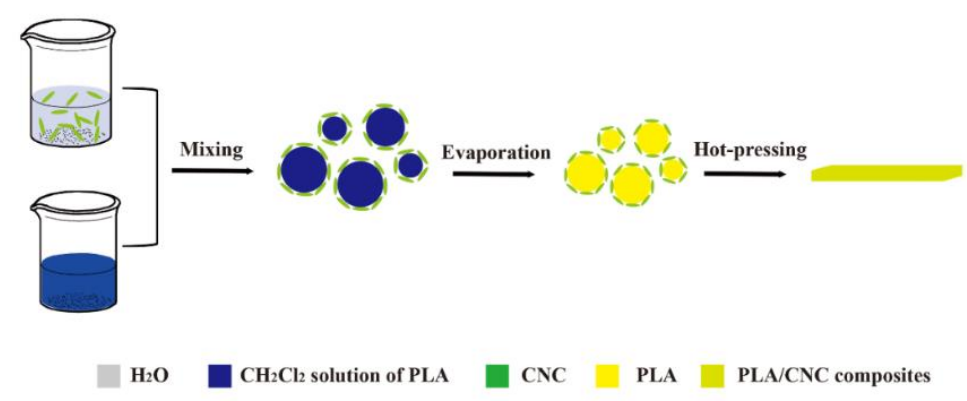

Figure 3.1 Schematic illustration of preparation of PLA/CNC composites via the Pickering emulsion approach.

\subsubsection{Preparation and characterization of $\mathrm{CNC}$}

CNC was prepared by the hydrogen chloride vapor approach ${ }^{16}$. Figure 3.2 shows the TEM image of CNC obtained in the procedure. The particles are typically rigid rod-shaped monocrystalline cellulose domains with about $15 \mathrm{~nm}$ in diameter and 360 $\mathrm{nm}$ in length. This result is consistent with previous work ${ }^{22}$. 


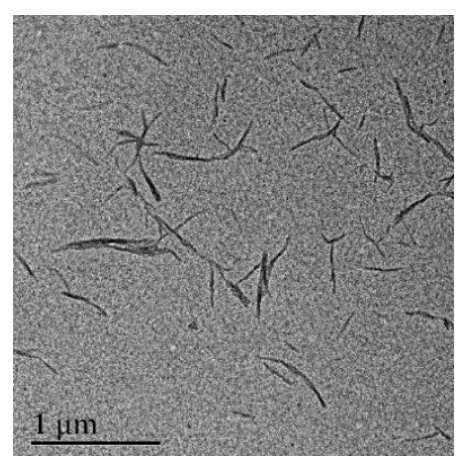

Figure 3.2 TEM image of CNC prepared by the hydrogen chloride vapor approach.

\subsubsection{Preparation of PLA/CNC composites}

The first step in the preparation of the PLA/CNC composites is the forming of an emulsion. The optical images of Pickering emulsions stabilized by different CNC contents are shown in Figure 3.3 The diameter of the oil droplets was under $50 \mu \mathrm{m}$ for all emulsions. The results indicated that a Pickering emulsion could be successfully prepared by using $\mathrm{CNC}$ as emulsifier.

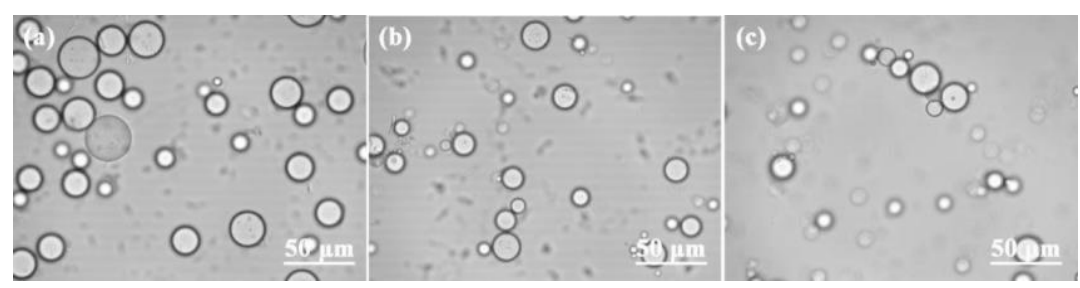

Figure 3.3 Images of Pickering emulsions stabilized by 5\% (a), 10\% (b) and 15\% CNC (c).

SEM images recorded on PLA/CNC composite microspheres with different CNC contents are presented in Figure 3.4. As is shown in Figure 3.4, precipitation resulted in PLA microspheres after filtration. After compression molding at $180^{\circ} \mathrm{C}$, the PLA microspheres reinforced with $\mathrm{CNC}$ melted to form the composites. 


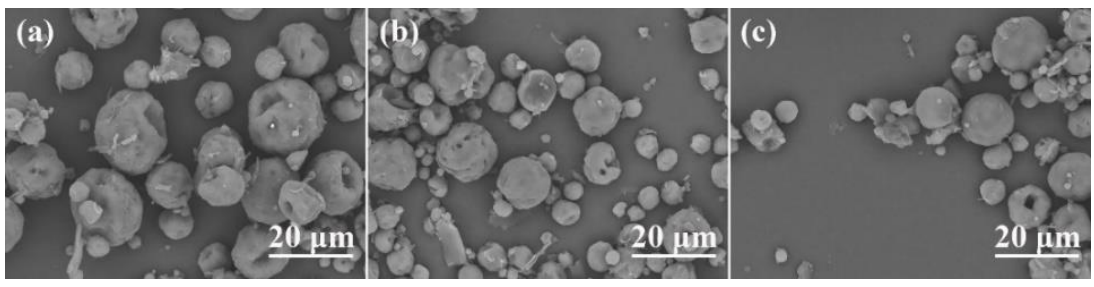

Figure 3.4 SEM images of PLA/CNC composites with (a) $5 \%$, (b) $10 \%$ and (c) $15 \% \mathrm{CNC}$ after filtration.

Figure 3.5 compares the $\mathrm{CNC}$ dispersion of the composites with 5 wt.\% CNC from different preparation approaches. In the Figure 3.5(a), extrusion of the freeze dried CNCs with neat PLA showed the formation of dark agglomerated particles, which is probably due to the degradation of $\mathrm{CNC}$ at high shear and temperature. The result is the same to the previous work ${ }^{23}$. The agglomerated particles were also observed in the composites prepared by solvent casting approach in the Figure 3.5(b). This is because the unmodified CNCs are very difficult to disperse in $\mathrm{CH}_{2} \mathrm{Cl}_{2}$, especially for high $\mathrm{CNC}$ contents. However, the composites prepared by Pickering emulsion approach was uniform and didn't produce any agglomerated particles. It showed the Pickering emulsion approach is a good way to prepare uniform PLA/CNC composites.
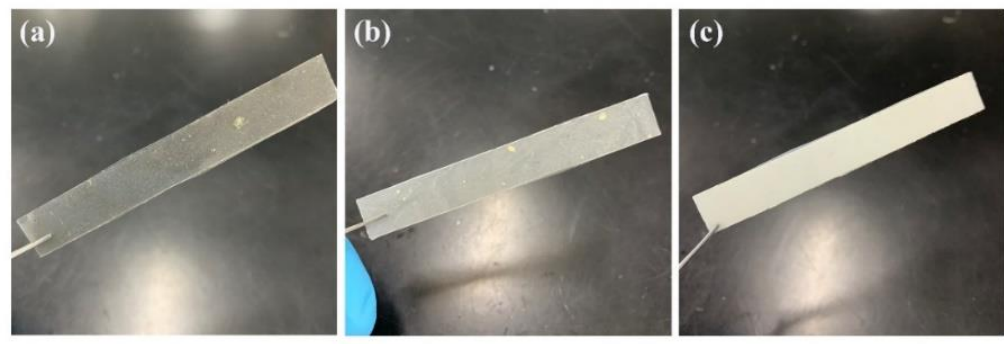

Figure 3.5 PLA/CNC composites prepared from (a) melt mixing approach, (b) solvent casting approach and Pickering emulsion approach.

The structure analysis of the composites was determined by using XRD and FTIR which are shown in Figure 3.6. In the Figure 3.6(a), The neat PLA shows a broad peak centered at around $2 \theta=16.5^{\circ}$, indicating PLA was predominantly amorphous. The XRD spectrum of PLA/CNC composites was a combination of those of CNC and 
PLA, indicating no changes took place from the compounding process in terms of crystallinity. Figure 3.6(b) showed the FT-IR spectra of the CNC, neat PLA and PLA/CNC composites. Compared to neat PLA, no obvious change was found for PLA/CNC composites, suggesting that there are no strong interactions between pristine CNC and PLA chain.
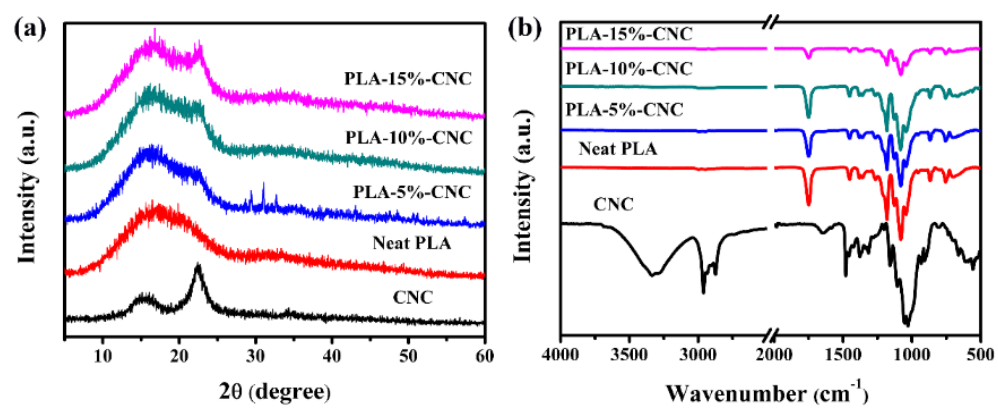

Figure 3.6 XRD analysis (a) and FT-IR spectra (b) of the CNC, neat PLA and PLA/CNC composites.

\subsubsection{Rheological characteristics}

Figure 3.7 presents the storage modulus $\left(G^{\prime}\right)$ and complex viscosity $\left(\eta^{*}\right)$ of neat PLA and that of the PLA/CNC composites as a function of frequency $(\omega)$ as determined in the rheological measurements. All samples exhibited stable rheological characteristics. As shown in Figure 3.7(a), the frequency dependence of storage modulus of neat PLA is typical for polymer melts. On the other hand, storage modulus increased and approached a plateau at low frequencies for the CNC composites. As shown in Figure 3.7(b), the complex viscosity of neat PLA displayed a long plateau, with a very slight shear-thinning behavior at high frequencies. However, all composites exhibited an obvious shear-thinning behavior without any plateau region at low frequencies. These results indicated that CNC may form a network in the PLA matrix thus promoting the transition from a fluid to a solid-like behavior at high temperatures as a result of the good dispersion of the CNC in the PLA matrix ${ }^{24-25}$. 

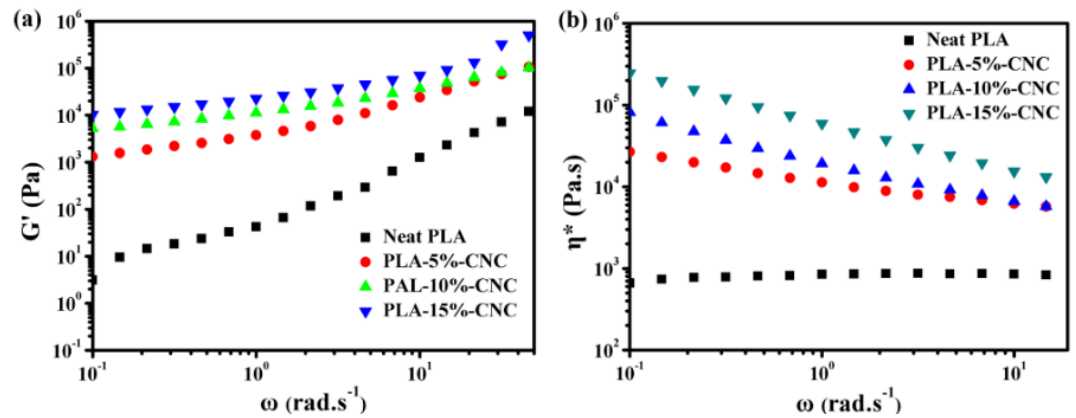

Figure 3.7 Values for (a) storage modulus and (b) complex viscosity of neat PLA and the PLA/CNC composites.

\subsubsection{Melting and crystallization}

The crystallization and melting behavior of PLA/CNC composites with various CNC contents were followed by DSC measurements. Figure 3.8 shows the DSC traces of neat PLA and the PLA/CNC composites. As shown in Fig. 8a, the exothermic crystallization of neat PLA appears as a broad peak with low intensity indicating the slow crystallization of the polymer ${ }^{26}$. As the CNC was added into the composites, the crystallization peak shifted to higher temperature and became more intense for the PLA/CNC composites as compared to neat PLA. CNC may act as nucleating agent ${ }^{27}$, but this assumption is contradicted by the fact that the increase in crystallization temperature does not depend on the amount of CNC in the composite. The presence of CNC might also increase the mobility of PLA chains as shown by previous research $^{28}$.
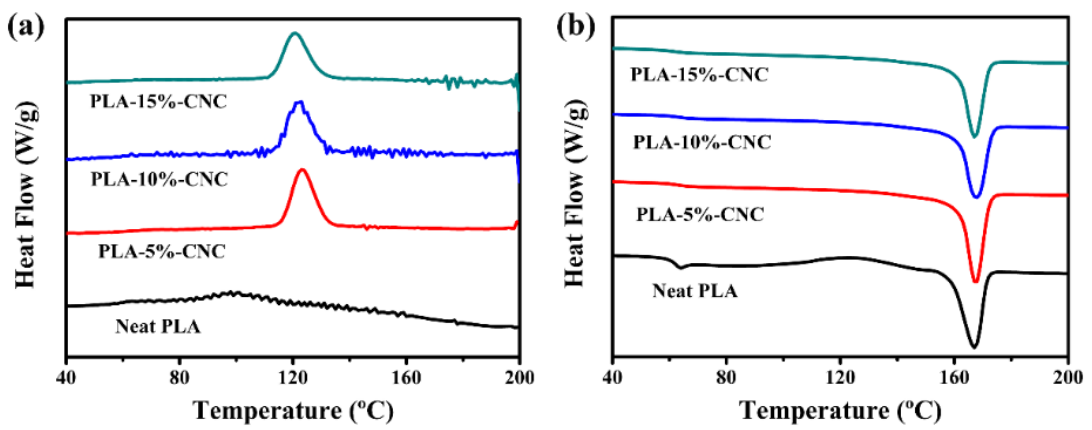

Figure 3.8 DSC traces during (a) cooling and (b) second heating scan of neat PLA and PLA/CNC composites.

In Figure 3.8(b) DSC heating traces are shown which were recorded on the 
PLA/CNC composites and on neat PLA. The traces show the glass transition of the polymer, followed by the cold crystallization and then the melting of the polymer. Crystallization is incomplete for the neat PLA during the cooling of the sample, because of the limited mobility of the molecules ${ }^{29}$. The cold crystallization peak disappears completely in the PLA/CNC composites and the melting peak becomes more intense upon the addition of CNC compared to neat PLA. All the changes indicate the increased rate of crystallization due to the presence of the cellulose nanocrystals ${ }^{30}$.

The crystallinity of the neat PLA and the composites were calculated from the second heating scan according to Equation 3.1 and the results are summarized in Table 3.1. The crystallinity of the polymer increased with increasing concentration of CNC in the PLA matrix. Compared to neat PLA, the crystallinity of the PLA-15\%CNC composite increased considerably, by more than $100 \%$. This result confirms previous observations related to the effect of $\mathrm{CNC}$ on the crystallization of PLA. According these results $\mathrm{CNC}$ could may act as nucleating agent or increase the rate of crystallization of the PLA matrix by improving the mobility of the polymer chains.

Table 3.1 Thermal characteristics of neat PLA and PLA/CNC composites derived from the second DSC heating scan.

\begin{tabular}{ccccccc}
\hline Sample & $\mathbf{T}_{\mathbf{g}}\left({ }^{\circ} \mathbf{C}\right)$ & $\mathbf{T}_{\mathbf{c c}}\left({ }^{\circ} \mathbf{C}\right)$ & $\mathbf{T}_{\mathbf{m}}\left({ }^{\circ} \mathbf{C}\right)$ & $\triangle \mathbf{H}_{\mathbf{c c}}(\mathbf{J} / \mathbf{g})$ & $\triangle \mathbf{H}_{\mathbf{m}}(\mathbf{J} / \mathbf{g})$ & $\chi(\mathbf{\%})$ \\
\hline Neat PLA & 60.1 & 122.2 & 167.0 & 15.8 & 36.2 & 21.9 \\
PLA-5\%-CNC & - & - & 167.5 & - & 37.5 & 42.3 \\
PLA-10\%-CNC & - & - & 167.9 & - & 36.9 & 43.6 \\
PLA-15\%-CNC & - & - & 167.1 & - & 35.8 & 44.3 \\
\hline
\end{tabular}

\subsubsection{Thermal decomposition, stability}

The thermal decomposition of PLA/CNC composites was determined by thermogravimetric analysis (TGA) in nitrogen atmosphere. The temperature corresponding to $5 \%$ weight loss is defined as the onset of thermal decomposition $\left(\mathrm{T}_{05 \%}\right)$. The TGA and the derivative TGA traces (DTG) are presented in Figure 3.9. Compared to the neat PLA, the $\mathrm{T}_{05 \%}$ of the composites shifted towards higher temperature. The $\mathrm{T}_{05 \%}$ temperature of neat PLA was about $321^{\circ} \mathrm{C}$ and it increased to $330^{\circ} \mathrm{C}$ for the PLA- $10 \%$-CNC composite indicating that the addition of $\mathrm{CNC}$ improved the thermal stability of the PLA/CNC composites. The increased thermal decomposition temperature of PLA/CNC composites can be attributed to char 
formation during the pyrolysis of $\mathrm{CNC}$ in the composites. The forming char acts as protective barrier that suppresses the thermal decomposition of the PLA matrix ${ }^{31}$. In addition, the amount of residues increased with increasing the concentration of CNC in the PLA matrix. This result may indicate that $\mathrm{CNC}$ promotes char formation indeed. The temperature belonging to the maximum rate of decomposition $\left(\mathrm{T}_{\max }\right)$ can be also determined from the DTG traces. The comparison of $\mathrm{T}_{\max }$ for the neat PLA and the composites indicates that this characteristic temperature does not change upon the addition of $\mathrm{CNC}$.
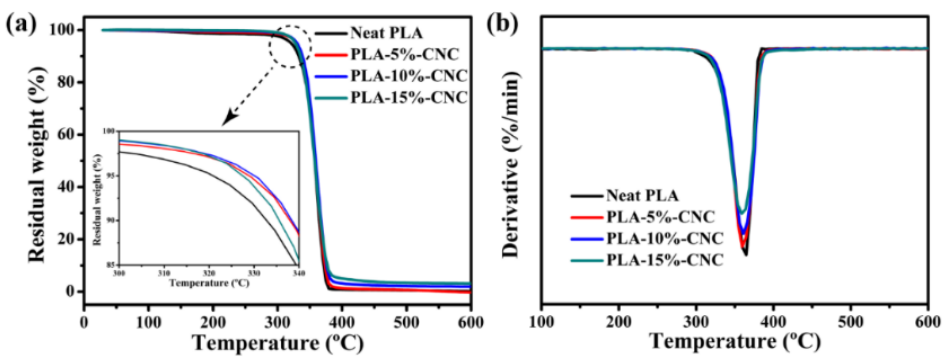

Figure 3.9 TGA (a) and DTG (b) traces of neat PLA and PLA/CNC composites.

\subsubsection{Dynamic mechanical analysis}

The thermomechanical properties of PLA/CNC composites and neat PLA were investigated by DMA to obtain information about the dynamic mechanical behavior of the composites with changing temperature. The storage modulus and the loss tangent of PLA/CNC composites and neat PLA are presented in Figure 3.10. The addition of $\mathrm{CNC}$ to the PLA matrix increased storage modulus considerably in the entire temperature range of the experiment indicating that $\mathrm{CNC}$ acts as efficient reinforcement in the polymer. In the glassy state, the storage modulus of the composites increases with increasing $\mathrm{CNC}$ content as a result of the reinforcing effect of $\mathrm{CNC}$ in the PLA matrix. Modulus decreases during the transition from the glassy state the rubbery state above $\mathrm{T}_{\mathrm{g}}$ at around $60{ }^{\circ} \mathrm{C}$, a considerable drop in storage modulus is observed for both the neat PLA and the PLA/CNC composites. Compared to the neat PLA, a substantial increase of storage modulus from $0.14 \mathrm{GPa}$ to $0.67 \mathrm{GPa}$ was observed for the PLA- $15 \%$-CNC composite at $70{ }^{\circ} \mathrm{C}$ due to the high crystallinity of the composites and the probable network formation of CNC in the PLA matrix ${ }^{32}$. Cold crystallization resulted in an increase in modulus studied above $90{ }^{\circ} \mathrm{C}$ for all the materials ${ }^{17}$. 

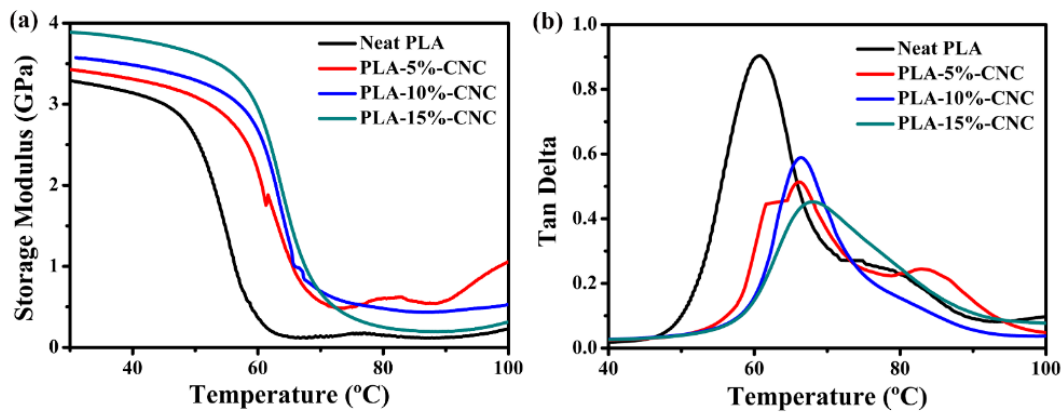

Figure 3.10 Effect of CNC content (wt.\%) on temperature dependence of (a) storage modulus and (b) loss tangent.

Figure 3.10(b) shows the changes in the position and intensity of the loss tangent peak as a function of temperature for the PLA/CNC composites and the neat PLA. The temperature of the peak may be assigned to the glass transition temperature $\left(\mathrm{T}_{\mathrm{g}}\right)$ of the polymer. Compared to neat PLA, the position of the peak shifted to higher temperatures in the composites. The $\mathrm{T}_{\mathrm{g}}$ of neat PLA was $60.8^{\circ} \mathrm{C}$, which increased to $68.1^{\circ} \mathrm{C}$ for the PLA-15\%-CNC composite indicating that $\mathrm{CNC}$ hinders the movement of chain segments in the PLA matrix ${ }^{33}$. However, this explanation contradicts the conclusion drawn from the melting and crystallization study showing increased molecular mobility in the presence of $\mathrm{CNC}$. A more probable explanation is increased crystallinity, which decreases the intensity of the loss tangent peak and shits it towards higher temperature. The decreased intensity indicates that fewer polymer chains participate in the transition as a consequence of the smaller relative amount of amorphous PLA phase, on the one hand, and due to the hindered mobility of these chains attached to the larger number of PLA crystals, on the other ${ }^{34}$.

\subsubsection{Mechanical properties}

The tensile properties of neat PLA the PLA/CNC composites with different CNC contents are shown in Figure 3.11. The tensile strength of the composites decreases with increasing the CNC content in the PLA matrix. A slight decrease of the tensile strength can be observed from 64 to $55 \mathrm{MPa}$ when adding 5\% CNC into the PLA matrix. The behavior of the composites is similar to that published in the literature ${ }^{35}$. Although the interaction between the PLA matrix ${ }^{36}$ and cellulose is quite strong ${ }^{37}$, the large stiffness of the matrix leads to the apparent decrease of strength. The real influence of $\mathrm{CNC}$ on mechanical properties and its load bearing capacity cannot be judged from the composition dependence of strength directly, it must be evaluated by 
model calculations. Figure 3.11(b) shows that the tensile modulus of the composites increases with increasing $\mathrm{CNC}$ content because of the good dispersion of $\mathrm{CNC}$ in the PLA matrix. Compared to neat PLA, an increase of the tensile modulus from 3.5 to 3.8 GPa was observed for the PLA-15\%-CNC composite. Similar improvement in tensile modulus was reported earlier ${ }^{32,38}$. The increase in modulus results from the reinforcing effect of the filler and from the increased crystallinity of the composites ${ }^{39-}$ 40 .
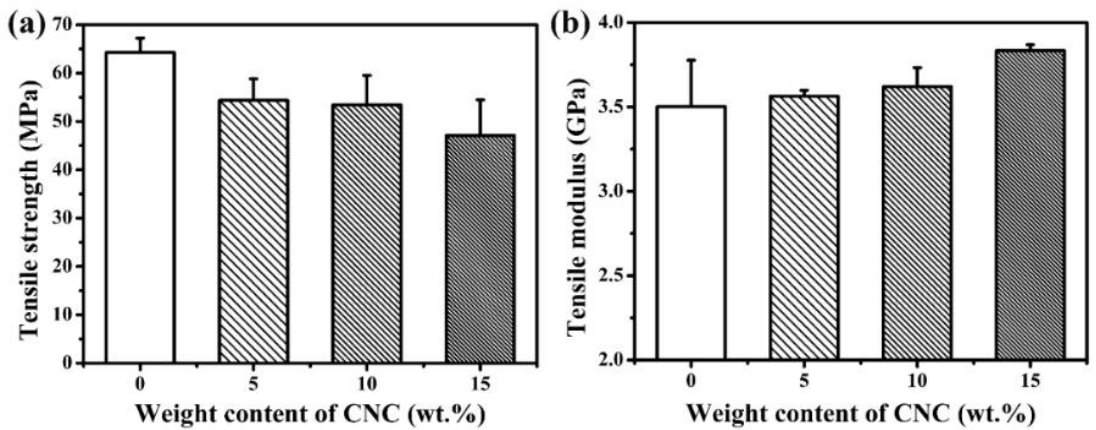

Figure 3.11 Values for (a) tensile strength and (b) modulus of neat PLA and PLA/CNC composites.

The flexural properties of neat PLA and the composites are shown in Figure 3.12. Compared to neat PLA, the flexural strength of the composite increased from 64 to $76 \mathrm{MPa}$ after adding 5\% CNC to the PLA matrix. Interestingly, flexural strength does not change with increasing $\mathrm{CNC}$ content probably because of the conflicting effect of filler content and the association of the nanocrystals. According to Figure 3.12(b), the flexural modulus of the composites increases with increasing CNC content, similarly to results obtained in tensile testing. The modulus was improved from 1.65 to 2.50 $\mathrm{GPa}$ at the CNC content of $15 \%$ compared to neat PLA. The increase of the crystallinity of the composites, the high aspect ratio of CNC and maybe also network formation all contributed to the improvement of flexural modulus ${ }^{41}$. 

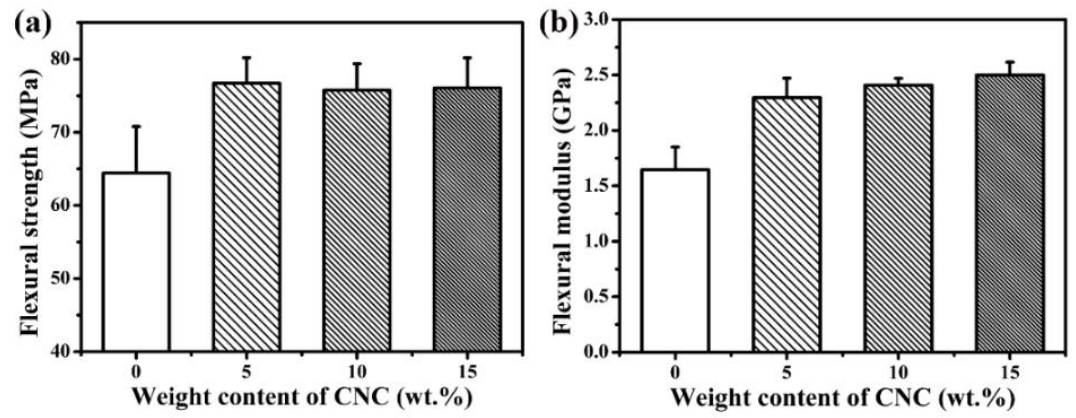

Figure 3.12 Composition dependence of the (a) flexural strength and (b) modulus of PLA/CNC composites.

\subsection{Conclusions}

PLA/CNC composites were prepared successfully by the Pickering emulsion approach. The rheological measurements indicated that CNC may form a network in the PLA matrix resulting in a transition from fluid to solid-like behavior at high temperatures resulting from the good dispersion of the CNC in the PLA matrix. DSC analysis confirmed that cellulose nanocrystals produced by hydrogen chloride vapor treatment increase the mobility of PLA chains and thus promote the crystallization of the polymer. The results of TG measurements indicated that $\mathrm{CNC}$ slightly increases the thermal decomposition temperature of the PLA matrix. Compared to neat PLA, the $\mathrm{T}_{05 \%}$ temperature of the composites increased from 321 to $330{ }^{\circ} \mathrm{C}$. Compared to the neat PLA the composites had increased storage modulus in the entire temperature range studied. At $70{ }^{\circ} \mathrm{C}$, a substantial increase from 0.14 to $0.67 \mathrm{GPa}$ was observed in storage modulus for the PLA-15\%-CNC composite. The tensile modulus of the PLA/CNC composites increases, while their tensile strength slightly decreases as compared to neat PLA. The flexural strength and modulus improved considerably upon the addition of CNC. The results unambiguously prove that unmodified CNC produced by the hydrochloric acid vapor approach improves efficiently the thermal, mechanical and rheological properties of PLA and thus it can be a promising reinforcement for polymer composites.

\subsection{Experimental sections}

\subsubsection{Materials}

PLA (2003D, D-lactic acid: 1.4\%, L-lactic acid: $98.6 \%$, density: $1.24 \mathrm{~g} / \mathrm{cm}^{3}$ ) with the number-average molecular weight $\left(\mathrm{M}_{\mathrm{n}}\right)$ of $\sim 150000 \mathrm{Da}$ and weight-average $\left(\mathrm{M}_{\mathrm{w}}\right)$ 
of 200000 Da, respectively, was supplied by Natureworks, USA. Wood Pulp was obtained from the Xinxiang Natural Chemical Co., Ltd. The viscosity-average degree of polymerization measured in a solution of cupric ethylene diamine hydroxide (CUEN) was estimated to be 870 . The measurement was done using an Ubbelohde viscometer. Dichloromethane $\left(\mathrm{CH}_{2} \mathrm{Cl}_{2}\right)$ and $37 \mathrm{wt} . \%$ hydrochloric acid $(\mathrm{HCl})$ were supplied by Sinopharm Chemical Reagent CO., Ltd.

\subsubsection{Preparation of CNC by the hydrogen chloride vapor approach}

The CNC was prepared by the method previously reported by Kontturi et $\mathrm{al}^{16}$. The hydrolysis was carried out in a vacuum desiccator with $35 \% \mathrm{HCl}$ solution poured onto the bottom. The desiccator valve was left open for more than a day in order to substitute the air inside the vessel by the $\mathrm{HCl}$ atmosphere completely. Subsequently, a wood pulp board was placed inside the desiccator and the hydrolysis was allowed to proceed for $8 \mathrm{~h}$ at $40^{\circ} \mathrm{C}$, after which the hydrolyzed pulp was transferred into water to rinse away excess $\mathrm{HCl}$. The hydrolyzed pulp was sonicated for 30 minutes followed by high-pressure homogenization at 800 bar for six times. The product was redispersed by ultrasonication for $10 \mathrm{~min}$. The concentration of cellulose was determined gravimetrically to be $1.01 \% \mathrm{w} / \mathrm{v}$ in the final dispersion.

\subsubsection{Preparation of PLA/CNC composites}

\section{Pickering emulsion approach}

A CNC dispersion was diluted with deionized water to obtain cellulose dispersions with the concentrations of $0.25,0.5$ and $0.75 \mathrm{w} / \mathrm{v} \%$. The CNC to PLA ratio $(5,10$ and $15 \mathrm{wt} . \%$ ) was adjusted to obtain the desired solid contents of $\mathrm{CNC}$ in the composites. $\mathrm{A} \mathrm{CH}_{2} \mathrm{Cl}_{2}$ solution of PLA $(10 \mathrm{ml}, 100 \mathrm{mg} / \mathrm{ml})$ was added to the aqueous dispersion of $\mathrm{CNC}$. The $\mathrm{CH}_{2} \mathrm{Cl}_{2}$ /water emulsion stabilized by $\mathrm{CNC}$ was formed by homogenization (IKA T18 homogenizer, Germany) at 12,000 rpm for $3 \mathrm{~min}$ followed by ultrasonication (Scientz JY 92-IIDN, China) for $3 \mathrm{~min}$. $\mathrm{CH}_{2} \mathrm{Cl}_{2}$ was evaporated at ambient temperature over 24 hours. The precipitates were vacuum filtered using a filter screen (500 mesh). The paste collected on the filter was vacuum dried at $60{ }^{\circ} \mathrm{C}$ for $24 \mathrm{~h}$. The PLA/CNC composites were produced by the compression molding of the dried paste using a Carver Laboratory Press (Carver Inc., USA) at $180{ }^{\circ} \mathrm{C}$ and $1500 \mathrm{~kg}$ for $5 \mathrm{~min}$. 
Solvent casting approach

The suspension of the CNCs was solvent exchange to acetone by centrifuging for 3 times in order to remove water (Thermo, USA). In the same way, the CNCs was solvent exchange to $\mathrm{CH}_{2} \mathrm{Cl}_{2}$. A predetermined amount of $\mathrm{CNC}$ suspension in $\mathrm{CH}_{2} \mathrm{Cl}_{2}$ was mixed with $\mathrm{CH}_{2} \mathrm{Cl}_{2}$ solution of PLA $(10 \mathrm{ml}, 100 \mathrm{mg} / \mathrm{ml})$. The mixture solutions were stirred for $4 \mathrm{~h}$ before casting. Then $\mathrm{CH}_{2} \mathrm{Cl}_{2}$ was allowed to evaporate off at ambient temperature for $24 \mathrm{~h}$. After that, the composite film could be prepared by using hot-pressing at $180^{\circ} \mathrm{C}$ for $5 \mathrm{~min}$ (Carver Inc., USA).

\section{Melting mixing approach}

The suspension of the CNCs was freeze dried for $36 \mathrm{~h}$ to get $\mathrm{CNC}$ powder (Labconco, USA). PLA granules and CNC powder were vacuum dried at $40{ }^{\circ} \mathrm{C}$ overnight to remove the adsorbed moisture. The PLA granules and CNC power were mixed in a beaker and extruded in a twin screw extruder (Ruiming SJZS-10J, China). The extrusion was carried out in the temperature range of $170-200{ }^{\circ} \mathrm{C}$ and the screw speed was held constant at $150 \mathrm{rpm}$. After that, the composite film could be prepared by using hot-pressing at $180^{\circ} \mathrm{C}$ for $5 \mathrm{~min}$ (Carver Inc., USA).

\subsubsection{Characterization}

\section{Microscopy}

The morphology of $\mathrm{CNC}$ was characterized using transmission electron microscopy (TEM, JEM-2100, Jeol, Japan) operated at $100 \mathrm{kV}$ voltage. TEM micrographs were recorded on a dried droplet of the aqueous suspension containing $0.05 \%$ of CNC using a carbon-coated grid (200 mesh).

After filtration, PLA/CNC composite microspheres were studied with scanning electron microscopy (SEM, TM-1000, Hitchi, Japan) at $2 \mathrm{kV}$ and 6-8 A. The samples were kept overnight in a vacuum oven at $40{ }^{\circ} \mathrm{C}$ and then coated with a gold-palladium alloy for $40 \mathrm{~s}$.

The emulsion obtained was observed using an optical microscope (Eclipse E100, Nikon, Japan).

\section{Rheology}

The rheological characteristics of neat PLA and the PLA/CNC composites were measured using a ThermoHaake-Mars 60 rheometer with parallel plate geometry (25 $\mathrm{mm}$ diameter) at $200{ }^{\circ} \mathrm{C}$. A frequency sweep from $0.01 \mathrm{~Hz}$ to $10 \mathrm{~Hz}$ was carried out 
to study the storage modulus $\left(G^{\prime}\right)$ and complex viscosity $\left(\eta^{*}\right)$ of the composites at the constant deformation of $1 \%$.

\section{Thermal analysis}

Neat PLA and the PLA/CNC composites were analyzed using differential scanning calorimetry (DSC, 214, Netzsch, Germany). The samples were heated from 25 to $200{ }^{\circ} \mathrm{C}$, held at $200{ }^{\circ} \mathrm{C}$ for $5 \mathrm{~min}$ to eliminate thermal history, cooled to $25^{\circ} \mathrm{C}$, and heated again to $200{ }^{\circ} \mathrm{C}$ under nitrogen purge. The heating rate was $10{ }^{\circ} \mathrm{C} / \mathrm{min}$, while the cooling rate $2{ }^{\circ} \mathrm{C} / \mathrm{min}$. Crystallization temperature $\left(\mathrm{T}_{\mathrm{c}}\right)$ and crystallization enthalpy $\left(\triangle \mathrm{H}_{\mathrm{c}}\right)$ were determined from the cooling scan. The glass transition temperature $\left(\mathrm{T}_{\mathrm{g}}\right)$, cold crystallization temperature $\left(\mathrm{T}_{\mathrm{cc}}\right)$, melting temperature $\left(\mathrm{T}_{\mathrm{m}}\right)$, cold crystallization enthalpy $\left(\triangle \mathrm{H}_{\mathrm{cc}}\right)$ and melting enthalpy $\left(\triangle \mathrm{H}_{\mathrm{m}}\right)$ were determined from the second heating scan.

$$
X(\%)=\left(\frac{\Delta H_{m}+\Delta H_{c c}}{\Delta \mathrm{H}_{\mathrm{m}}^{0} *\left(1-\frac{w t . \% \text { filler }}{100}\right)}\right) * 100
$$

\section{Equation 3.1}

Where $\triangle \mathrm{H}_{\mathrm{m}}^{0}$ is the melting enthalpy of a $100 \%$ crystalline polymer matrix $(93.0$ $\mathrm{J} / \mathrm{g}$ ) for PLA, and wt.\% filler is the weight percentage of CNC.

The thermal decomposition of neat PLA and the PLA/CNC composites were evaluated by thermogravimetric analysis (TG, Netzsch 209F3, Germany). The samples were heated from 30 to $600{ }^{\circ} \mathrm{C}$ under nitrogen purge. The heating rate was $10{ }^{\circ} \mathrm{C} / \mathrm{min}$.

Dynamic mechanical analysis (DMA) was performed in single cantilever mode using a TA Instruments Q800 analyzer (New Castle, DE). The dimensions of the test samples were $35 \times 10 \times 0.5 \mathrm{~mm}$ (length $\times$ width $\times$ thickness). The measurements were done at constant frequency $(1 \mathrm{~Hz})$ and amplitude $(15 \mu \mathrm{m})$ in the temperature range from 30 to $120{ }^{\circ} \mathrm{C}$ with the heating rate of $3{ }^{\circ} \mathrm{C} / \mathrm{min}$.

\section{Mechanical testing}

The tensile properties of neat PLA and the PLA/CNC composites were determined using a universal testing machine (UH6502, Youhong, China). The experiments were done at a gauge length of $30 \mathrm{~mm}$ and a crosshead speed of $10 \mathrm{~mm} / \mathrm{min}$. Five specimens were measured for each set of samples. The samples were thin rectangular plates with the dimensions of $100 \times 20 \times 0.5 \mathrm{~mm}$.

Flexural testing was conducted using a universal testing machine (UH6502, Youhong, China) on rectangular specimens $\left(50.8 \times 12.7 \times 0.5 \mathrm{~mm}^{3}\right)$ at a support span of $25.4 \mathrm{~mm}$ and a crosshead speed of $5 \mathrm{~mm} / \mathrm{min}$ according to the ASTM D 790-2007 
standard.

$X$-ray diffraction $(X R D)$

The CNC, neat PLA and PLA/CNC composites were analyzed using a Rigaku Xray diffractor (D/max 2550PC, Japan). The samples were exposed for $1.5 \mathrm{~s}$ for each angle of incidence $(\theta)$ using a $\mathrm{Cu} \mathrm{K \alpha} \mathrm{X}$-ray source with a wavelength of $1.541 \AA$. The angle of incidence is varied from 4 to 50 by steps of $0.02 \mathrm{~s}$. The crystallinity index (CI, \%) was estimated from the intensities of characteristic peaks.

Fourier transform infrared spectroscopy (FT-IR)

The CNC, neat PLA and PLA/CNC composites were performed on a PerkinElmer Spectrum-Two (American) equipped with an attenuated total reflectance (ATR) accessory. The transmittance of the infrared in individual sample of substrate was recorded from 4000.0 to $500.0 \mathrm{~cm}^{-1}$ at a resolution of $2.0 \mathrm{~cm}^{-1}$ for infrared spectra 


\section{References}

[1] Finkenstadt, V. L.; Liu, C.-K.; Evangelista, R.; Liu, L.; Cermak, S. C.; HojillaEvangelista, M.; Willett, J. Poly (lactic acid) green composites using oilseed coproducts as fillers. Industrial Crops and Products 2007, 26 (1), 36-43.

[2] Rasal, R. M.; Janorkar, A. V.; Hirt, D. E. Poly (lactic acid) modifications. Progress in Polymer Science 2010, 35 (3), 338-356.

[3] Spinella, S.; Re, G. L.; Liu, B.; Dorgan, J.; Habibi, Y.; Leclere, P.; Raquez, J.-M.; Dubois, P.; Gross, R. A. Polylactide/cellulose nanocrystal nanocomposites: Efficient routes for nanofiber modification and effects of nanofiber chemistry on PLA reinforcement. Polymer 2015, 65, 9-17.

[4] Kian, L.; Saba, N.; Jawaid, M.; Sultan, M. A review on processing techniques of bast fibers nanocellulose and its polylactic acid (PLA) nanocomposites. International Journal of Biological Macromolecules 2018, 121, 1314-1328.

[5] Luo, W.; Cheng, L.; Yuan, C.; Wu, Z.; Yuan, G.; Hou, M.; Chen, J. Y.; Luo, C.; Li, W. Preparation, characterization and evaluation of cellulose nanocrystal/poly (lactic acid) in situ nanocomposite scaffolds for tissue engineering. International Journal of Biological Macromolecules 2019, 134, 469-479.

[6] Zhou, C.; Shi, Q.; Guo, W.; Terrell, L.; Qureshi, A. T.; Hayes, D. J.; Wu, Q. Electrospun bio-nanocomposite scaffolds for bone tissue engineering by cellulose nanocrystals reinforcing maleic anhydride grafted PLA. ACS Applied Materials \& Interfaces 2013, 5 (9), 3847-3854.

[7] Klemm, D.; Heublein, B.; Fink, H. P.; Bohn, A. Cellulose: fascinating biopolymer and sustainable raw material. Angewandte Chemie International Edition 2005, 44 (22), 3358-3393.

[8] Klemm, D.; Kramer, F.; Moritz, S.; Lindström, T.; Ankerfors, M.; Gray, D.; Dorris, A. Nanocelluloses: a new family of nature-based materials. Angewandte Chemie International Edition 2011, 50 (24), 5438-5466.

[9] Moon, R. J.; Martini, A.; Nairn, J.; Simonsen, J.; Youngblood, J. Cellulose nanomaterials review: structure, properties and nanocomposites. Chemical Society Reviews 2011, 40 (7), 3941-3994.

[10] Silvério, H. A.; Neto, W. P. F.; Dantas, N. O.; Pasquini, D. Extraction and characterization of cellulose nanocrystals from corncob for application as reinforcing agent in nanocomposites. Industrial Crops and Products 2013, 44, 
427-436.

[11] Wang, Z.; Yao, Z.; Zhou, J.; He, M.; Jiang, Q.; Li, A.; Li, S.; Liu, M.; Luo, S.; Zhang, D. Improvement of polylactic acid film properties through the addition of cellulose nanocrystals isolated from waste cotton cloth. International Journal of Biological Macromolecules 2019, 129, 878-886.

[12] Shojaeiarani, J.; Bajwa, D. S.; Stark, N. M.; Bajwa, S. G. Rheological properties of cellulose nanocrystals engineered polylactic acid nanocomposites. Composites Part B: Engineering 2019, 161, 483-489.

[13] Azizi Samir, M. A. S.; Alloin, F.; Dufresne, A. Review of recent research into cellulosic whiskers, their properties and their application in nanocomposite field. Biomacromolecules 2005, 6 (2), 612-626.

[14] de Souza Lima, M. M.; Borsali, R. Rodlike cellulose microcrystals: structure, properties, and applications. Macromolecular Rapid Communications 2004, 25 (7), 771-787.

[15] Haafiz, M. M.; Hassan, A.; Khalil, H. A.; Fazita, M. N.; Islam, M. S.; Inuwa, I.; Marliana, M.; Hussin, M. H. Exploring the effect of cellulose nanowhiskers isolated from oil palm biomass on polylactic acid properties. International Journal of Biological Macromolecules 2016, 85, 370-378.

[16] Kontturi, E.; Meriluoto, A.; Penttilä, P. A.; Baccile, N.; Malho, J. M.; Potthast, A.; Rosenau, T.; Ruokolainen, J.; Serimaa, R.; Laine, J. Degradation and crystallization of cellulose in hydrogen chloride vapor for high-yield isolation of cellulose nanocrystals. Angewandte Chemie International Edition 2016, 55 (46), 14455-14458.

[17] Lin, N.; Huang, J.; Chang, P. R.; Feng, J.; Yu, J. Surface acetylation of cellulose nanocrystal and its reinforcing function in poly (lactic acid). Carbohydrate Polymers 2011, 83 (4), 1834-1842.

[18] Goffin, A.-L.; Raquez, J.-M.; Duquesne, E.; Siqueira, G.; Habibi, Y.; Dufresne, A.; Dubois, P. From interfacial ring-opening polymerization to melt processing of cellulose nanowhisker-filled polylactide-based nanocomposites. Biomacromolecules 2011, 12 (7), 2456-2465.

[19] Mougel, J. B.; Bertoncini, P.; Cathala, B.; Chauvet, O.; Capron, I. Macroporous hybrid Pickering foams based on carbon nanotubes and cellulose nanocrystals. Journal of Colloid and Interface Science 2019, 544, 78-87. 
[20] Zhang, Y.; Jiang, Y.; Han, L.; Wang, B.; Xu, H.; Zhong, Y.; Zhang, L.; Mao, Z.; Sui, X. Biodegradable regenerated cellulose-dispersed composites with improved properties via a Pickering emulsion process. Carbohydrate Polymers 2018, 179, 86-92.

[21] Zhang, Y.; Wu, J.; Wang, B.; Sui, X.; Zhong, Y.; Zhang, L.; Mao, Z.; Xu, H. Cellulose nanofibril-reinforced biodegradable polymer composites obtained via a Pickering emulsion approach. Cellulose 2017, 24 (8), 3313-3322.

[22] Lorenz, M.; Sattler, S.; Reza, M.; Bismarck, A.; Kontturi, E. Cellulose nanocrystals by acid vapour: towards more effortless isolation of cellulose nanocrystals. Faraday Discussions 2017, 202, 315-330.

[23] Dhar, P.; Tarafder, D.; Kumar, A.; Katiyar, V. Thermally recyclable polylactic acid/cellulose nanocrystal films through reactive extrusion process. Polymer 2016, $87,268-282$.

[24] Bagheriasl, D.; Carreau, P. J.; Riedl, B.; Dubois, C.; Hamad, W. Y. Shear rheology of polylactide (PLA)-cellulose nanocrystal (CNC) nanocomposites. Cellulose 2016, 23 (3), 1885-1897.

[25] Kamal, M. R.; Khoshkava, V. Effect of cellulose nanocrystals (CNC) on rheological and mechanical properties and crystallization behavior of PLA/CNC nanocomposites. Carbohydrate Polymers 2015, 123, 105-114.

[26] Saeidlou, S.; Huneault, M. A.; Li, H.; Park, C. B. Poly (lactic acid) crystallization. Progress in Polymer Science 2012, 37 (12), 1657-1677.

[27]Fortunati, E.; Armentano, I.; Zhou, Q.; Iannoni, A.; Saino, E.; Visai, L.; Berglund, L. A.; Kenny, J. Multifunctional bionanocomposite films of poly (lactic acid), cellulose nanocrystals and silver nanoparticles. Carbohydrate Polymers 2012, 87 (2), 1596-1605.

[28] Müller, P.; Imre, B.; Bere, J.; Móczó, J.; Pukánszky, B. Physical ageing and molecular mobility in PLA blends and composites. Journal of Thermal Analysis and Calorimetry 2015, 122 (3), 1423-1433.

[29] Suryanegara, L.; Nakagaito, A. N.; Yano, H. Thermo-mechanical properties of microfibrillated cellulose-reinforced partially crystallized PLA composites. Cellulose 2010, 17 (4), 771-778.

[30] Suryanegara, L.; Nakagaito, A. N.; Yano, H. The effect of crystallization of PLA on the thermal and mechanical properties of microfibrillated cellulose-reinforced 
PLA composites. Composites Science and Technology 2009, 69 (7-8), 1187-1192.

[31] Perinović, S.; Andričić, B.; Erceg, M. Thermal properties of poly (1-lactide)/olive stone flour composites. Thermochimica Acta 2010, 510 (1-2), 97-102.

[32] Jonoobi, M.; Harun, J.; Mathew, A. P.; Oksman, K. Mechanical properties of cellulose nanofiber (CNF) reinforced polylactic acid (PLA) prepared by twin screw extrusion. Composites Science and Technology 2010, 70 (12), 1742-1747.

[33] Tomé, L. C.; Pinto, R. J.; Trovatti, E.; Freire, C. S.; Silvestre, A. J.; Neto, C. P.; Gandini, A. Transparent bionanocomposites with improved properties prepared from acetylated bacterial cellulose and poly (lactic acid) through a simple approach. Green Chemistry 2011, 13 (2), 419-427.

[34] Tingaut, P.; Zimmermann, T.; Lopez-Suevos, F. Synthesis and characterization of bionanocomposites with tunable properties from poly (lactic acid) and acetylated microfibrillated cellulose. Biomacromolecules 2009, 11 (2), 454-464.

[35] Robles, E.; Urruzola, I.; Labidi, J.; Serrano, L. Surface-modified nano-cellulose as reinforcement in poly (lactic acid) to conform new composites. Industrial Crops and Products 2015, 71, 44-53.

[36] Petersson, L.; Oksman, K. Biopolymer based nanocomposites: comparing layered silicates and microcrystalline cellulose as nanoreinforcement. Composites Science and Technology 2006, 66 (13), 2187-2196.

[37] Faludi, G.; Dora, G.; Imre, B.; Renner, K.; Móczó, J.; Pukánszky, B. PLA/lignocellulosic fiber composites: particle characteristics, interfacial adhesion, and failure mechanism. Journal of Applied Polymer Science 2014, 131 (4), 39902.

[38] Sung, S. H.; Chang, Y.; Han, J. Development of polylactic acid nanocomposite films reinforced with cellulose nanocrystals derived from coffee silverskin. Carbohydrate Polymers 2017, 169, 495-503.

[39] Haafiz, M. M.; Hassan, A.; Zakaria, Z.; Inuwa, I. M.; Islam, M. S.; Jawaid, M. Properties of polylactic acid composites reinforced with oil palm biomass microcrystalline cellulose. Carbohydrate Polymers 2013, 98 (1), 139-145.

[40] Siqueira, G.; Bras, J.; Dufresne, A. Cellulose whiskers versus microfibrils: influence of the nature of the nanoparticle and its surface functionalization on the thermal and mechanical properties of nanocomposites. Biomacromolecules 2008, 10 (2), 425-432. 
[41] Dasan, Y.; Bhat, A.; Ahmad, F. Polymer blend of PLA/PHBV based bionanocomposites reinforced with nanocrystalline cellulose for potential application as packaging material. Carbohydrate Polymers 2017, 157, 13231332 


\section{Chapter 4}

\section{Making Polymers Colored and Stiffer by Dyed Nanocellulose Employing Pickering Emulsions}

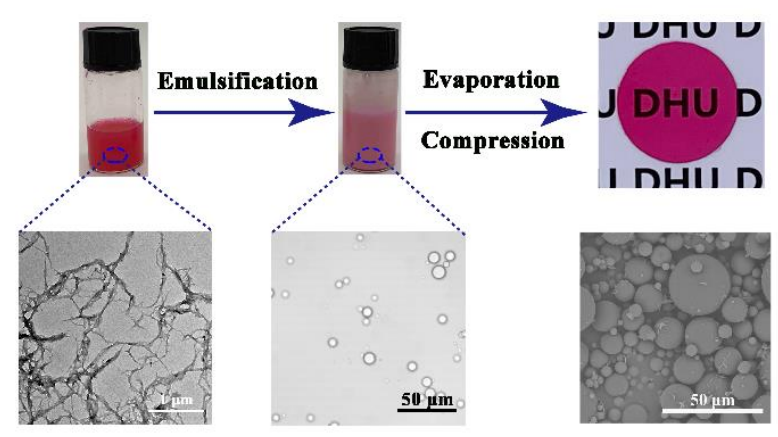

We demonstrate the successful use of Pickering emulsions utilizing nanocellulose that carry covalently immobilized dye molecules as emulsifiers to color PMMA and enhance its mechanical performance. Due to the use of this biobased dye carrier, the materials we describe exhibit enhanced "green" character. First we show that oil-in- 
water Pickering emulsions of PMMA can be effectively stabilized by colored nanocellulose. We describe how uniformly colored PMMA-cellulose composites with high transparency in the visible range can be obtained. We provide detailed characterization of the resulting composites. Thermogravimetric analysis shows an increase in onset of degradation temperature, thus indicates improved thermal stability. The mechanical properties are also improved, as indicated by flexural strength enhancement. The Pickering emulsion-based procedure is simple and cost effective, and could be easily adopted to prepare other polymer-nanocomposite systems.

\footnotetext{
*The contents of this chapter have been published as: Yunchong Zhang, Lei Ding, Binbin Ming, Bijia Wang, Xueling Feng, G. Julius Vancso and Xiaofeng Sui; Making Polymers Colored and Stiffer by Dyed Nanocellulose Employing Pickering Emulsions; Colloids and Surfaces A: Physicochemical and Engineering Aspects, 2020, 592, 124601.
} 


\subsection{Introduction}

The use of pigments in polymers (plastics, coatings and bulk products) is as old as the polymer industry ${ }^{1}$. Pigments have been used for decorative, as well as functional purposes. Recent progress in high value added applications, like security printing or angle-dependent automotive decorative paints, have added further interest in the continuing development of this field ${ }^{2}$.

However, due to the often different chemical nature of the pigment and the polymer matrix to be colored, molecular incompatibility and immiscibility effects often make it difficult to obtain well dispersed and stable pigments for a given polymer. To tackle this challenge, surfactants or encapsulation can be used. For example, miniemulsion polymerization can provide designer "pigment containers", and surface functionalization of the emulgeated particle can be tuned to achieve compatibilization with the matrix ${ }^{3}$.

Another way to obtain color in polymers is related to coupling of dye molecules to suitable carriers. Here we describe a strategy to use environmentally friendly nanocellulose as dye carrier in Pickering emulsions. Nanocellulose not only provides a surface active component as emulsifier and as carrier of dye molecules, but also adds strength (improves mechanical performance) to the polymer when it is used in composite systems following solvent removal. As active agent, we chose a dyed nanocellulose (CRC), which is a promising environmentally friendly, coloring material $^{4-5}$. As matrix we chose polymethyl methacrylate (PMMA), which is a glassy polymer with excellent transparency and good processing ability, and is commonly used in the transportation sectors, and as building materials, special application windows and body implants ${ }^{6-8}$. Our primary objective is not to offer a new type of PMMA, but demonstrate the use of Pickering emulsions in nanocomposite preparations for simultaneous mechanical performance improvement and coloring.

The combination of PMMA with nanocellulose ${ }^{9-10}$ has been discussed in the literature. Results demonstrated increase of mechanical strength and stiffness of PMMA composites while still retaining good optical transparency ${ }^{11}$. The optical properties of PMMA/nanocellulose composites are greatly influenced by the quality of the dispersion of nanocellulose in the matrix. Poor dispersion will severely compromise the transparency and mechanical properties of the composite even at very low loadings ${ }^{12}$. Previously, we have shown that cellulose nanofibers ${ }^{13}$, regenerated cellulose $(\mathrm{RC})^{14}$, and lignin nanoparticle ${ }^{15}$ could be evenly dispersed into polylactic acid (PLA) via a Pickering emulsion approach, where the cellulose nanoparticles 
served as the solid emulsifiers for $\mathrm{O} / \mathrm{W}$ emulsions. Upon slow evaporation of $\mathrm{CH}_{2} \mathrm{Cl}_{2}$, microspheres of PLA composites could be obtained, which were then hot pressed at $180^{\circ} \mathrm{C}$ to yield composite PLA films with enhanced properties.

As mentioned, here we present the preparation of highly transparent composites of PMMA and CRC with favorable color and mechanical properties via the Pickering emulsion route as a case study to demonstrate the feasibility of our successful "dual approach" (enhancing mechanical performance, and uniformly dispersing a pigment). $\mathrm{CRC}$ acted as the stabilizer and dye carrier in the oil-in-water Pickering emulsion featuring PMMA in the hydrophobic phase. Evenly colored composite PMMA/CRC microspheres obtained after solvent evaporation were further converted to films by compression molding. Thermal and mechanical characterization of these nanocomposites was performed. In comparison with common coloration methods of melt blending in colorants ${ }^{16}$, this approach could add bright color and mechanical strength to PMMA without compromising its transparency.

\subsection{Results and Discussion}

\subsubsection{PMMA/CRC Composites}

CRC suspensions were prepared by reacting $\mathrm{RC}$ with reactive dyes (RR24) in deionized water. The dyeing mechanism is the same as that of reactive dyeing of cotton fabrics. The coupling chemistry to covalently attach the dye to cellulose is shown in Figure 4.1 (a). TEM images of RC and CRC are shown in Figure 4.1. The fibrillar diameter of the RC and CRC (bearing covalently coupled dye) were in the nanometer range. The morphology of $\mathrm{RC}$ and $\mathrm{CRC}$ were similar, both of which exhibited a networked structure. Therefore, the morphology of RC was not changed by the dyeing process. CRC has good dispersion stability in water due to the hydrophilicity of cellulose. The CRC suspension could be stored over prolonged periods without precipitation as reported previously ${ }^{4-5}$. 

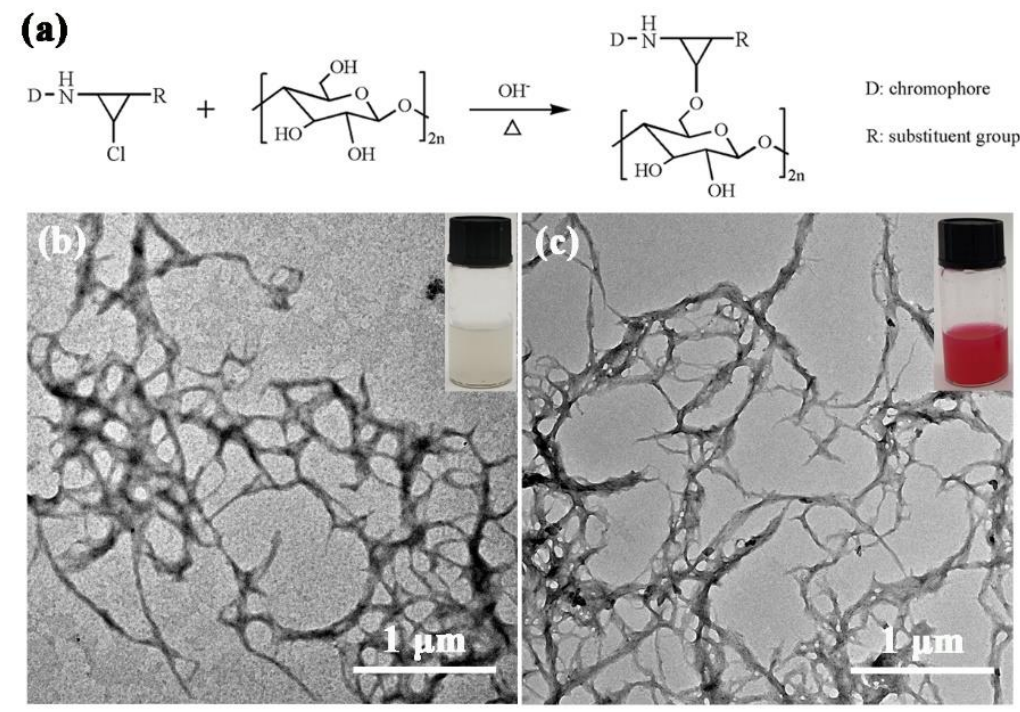

Figure 4.1 (a) The process of CRC dyeing, TEM images of (b) RC and (c) CRC.

$\mathrm{RC}$ is a good stabilizer to prepare Pickering emulsions due to the combination of the Pickering and the networking mechanisms ${ }^{17-18}$. Dyed CRC used in this study performed also very well as stabilizer to prepare colored Pickering emulsions. When the content of $\mathrm{CRC}$ was below $1 \%$, Pickering emulsions were not formed due to the insufficient quantity of CRC. Optical micrographs of Pickering emulsions stabilized by varying CRC content are displayed in Figure 4.2. It can be clearly seen that the oil droplets containing PMMA were well dispersed in the aqueous phase and the diameter values of the oil droplets were less than $50 \mu \mathrm{m}$ for all emulsions, indicate the effective stabilizing ability of CRC. We note that this procedure is not limited to PMMA; other emulsions with CRC could also be similarly obtained when needed.

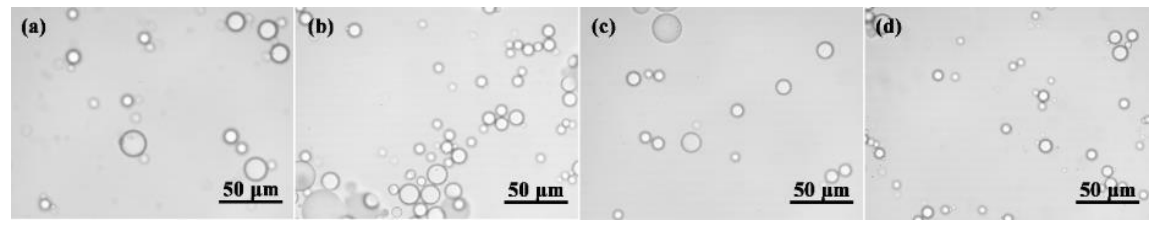

Figure 4.2 The optical microscope images of Pickering emulsion stabilized by (a) $1 \%$ CRC, (b) $2 \%$ CRC, (c) $3 \%$ CRC and (d) $4 \%$ CRC.

Colored PMMA microspheres were prepared during slowly evaporating the DCM solution from the emulsion at room temperature. SEM images of the resulting 
PMMA/CRC composite microspheres with different CRC contents are presented in Figure 4.3. The microspheres have diameters below $50 \mu \mathrm{m}$, which were similar in size to the oil droplets in the corresponding emulsions (see Figure 4.2 (a-d)). When the content of CRC was low, the microspheres had a relatively smooth surface. As more CRC was used, the surface roughness appeared to increase, likely due to the surface deposition of excess CRC.

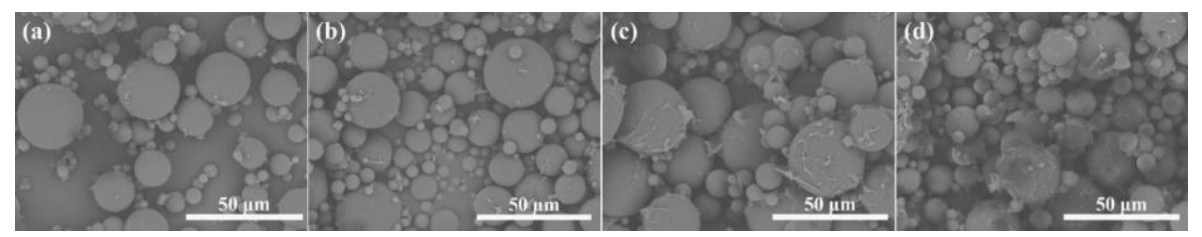

Figure 4.3 SEM images of PMMA microspheres with (a) $1 \%$ CRC, (b) $2 \%$ CRC, (c) $3 \%$ CRC and (d) $4 \%$ CRC.

Colored PMMA/CRC composite films were obtained by hot-pressing and thus fusing these colored composite microspheres. Figure 4.4(a) show the images of neat compressed PMMA, and the colored PMMA/CRC composite films with different CRC (and thus dye) loadings. The printed letters in the background can be seen through, indicating that all composites prepared here were visibly transparent. (We note thatlater we show, the optical transmittance values in the visible spectrum decreased with increasing dye content.) Visual inspection of the photographs in Figure 4.4(a) confirm that the color of the composite film was even and uniform without noticeable $\mathrm{CRC}$ aggregates, indicating that $\mathrm{CRC}$ was uniformly dispersed in the PMMA matrix throughout the concentration range studied. Obviously, darker color tones could be achieved as more CRC was incorporated into the PMMA matrix, showing the color depth of the composites are tunable by adjusting the CRC (and thus the dye) contents.

Figure 4.4(b) shows the quantitatively measured profiles of light transmittance versus the wavelength of visible light for the neat PMMA matrix and color PMMA/CRC composites examined by a UV-vis spectrometer at a visible wavelength range between 400-800 $\mathrm{nm}$. As expected, the neat PMMA showed the highest transmission (about 90\%). The transmittance spectra of the composite exhibited reduced values with increasing $\mathrm{CRC}$ (dye) loading due to structural heterogeneity (scattering) in line with earlier reports ${ }^{19-20}$. Figure 4.4(c) shows the difference of transmittance in the spectral absorption wavelength at $550 \mathrm{~nm}$, which indicated the 
increasing color depth of the composites with increased CRC loading.

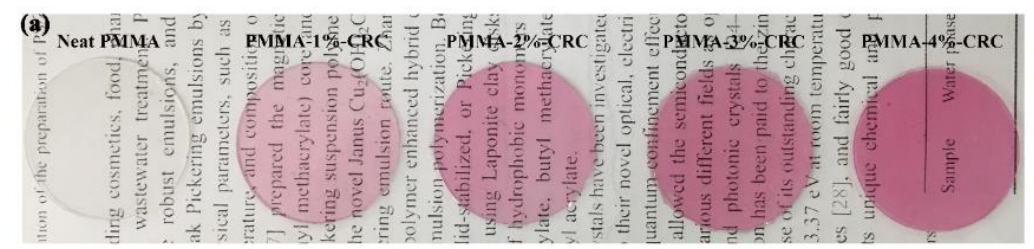

(b)

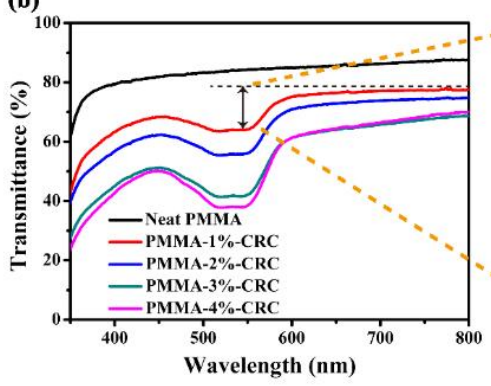

(c)

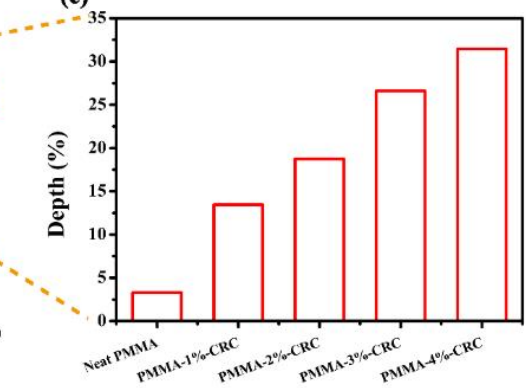

Figure 4.4 (a) Photograph of compressed PMMA nanocomposites with different CRC loading, (b) UV-Vis spectra and (c) color depth (from $550 \mathrm{~nm}$ to $800 \mathrm{~nm}$ ) of the colored PMMA/CRC composites with different CRC contents.

\subsubsection{Thermal Properties of PMMA/CRC Composites}

The thermal decomposition of PMMA/CRC composites was monitored by thermogravimetric analysis (TGA) in nitrogen atmosphere. The resulted TG and DTG thermograms are presented in Figure 4-S1 (in the Appendix). The onset of thermal decomposition ( $\left.\mathrm{T}_{\text {onset }}\right)$ and the maximum of thermal decomposition $\left(\mathrm{T}_{\max }\right)$ of the composites were summarized in Table 1. $\mathrm{T}_{\text {onset }}$ and $\mathrm{T}_{\max }$ shifted to higher temperatures as more CRC were incorporated into PMMA demonstrating enhanced thermal stability of the colored PMMA composites. As is shown in the Figure 4-S1, the thermal decomposition temperature of CRC was around $330{ }^{\circ} \mathrm{C}$, which had a lower value than the thermal decomposition temperature of the PMMA matrix. This could be explained by assuming that the char that formed during CRC pyrolysis could act as protective barrier that suppresses the thermal decomposition of the PMMA matrix ${ }^{21}$. There was no residual mass obtained for neat PMMA and the composites, which was attributed to the degradation of PMMA starting slowly at $220{ }^{\circ} \mathrm{C}$ and reached completion at a temperature higher than $360{ }^{\circ} \mathrm{C}^{22}$. 
Table 1. $\mathrm{T}_{\text {onset }}$ and $\mathrm{T}_{\max }$ of the neat PMMA and PMMA/CRC composites.

\begin{tabular}{ccc}
\hline Sample & $\mathbf{T}_{\text {onset }}\left({ }^{\circ} \mathbf{C}\right)$ & $\mathbf{T}_{\max }\left({ }^{\circ} \mathbf{C}\right)$ \\
\hline Neat PMMA & 366.8 & 389.4 \\
PMMA-1\%-CRC & 373.2 & 391.6 \\
PMMA-2\%-CRC & 384.7 & 390.6 \\
PMMA-3\%-CRC & 386.2 & 391.1 \\
PMMA-4\%-CRC & 390.7 & 395.3 \\
\hline
\end{tabular}

The DSC thermograms of neat PMMA and PMMA/CRC composites with different CRC contents were shown in Figure 4-S2 (in the Appendix). No obvious crystallization of any of the constituents was observed, which is consistent with the previous work $^{23}$. Glass transition temperature values $\left(\mathrm{T}_{\mathrm{g}}\right)$ were taken as the midpoint of the endothermic transition. The $\mathrm{T}_{\mathrm{g}}$ of neat PMMA was $101.1{ }^{\circ} \mathrm{C}$ while the $\mathrm{T}_{\mathrm{g}}$ of PMMA-1\%-CRC, PMMA-2\%-CRC, PMMA-3\%-CRC and PMMA-4\%-CRC were $99.7{ }^{\circ} \mathrm{C}, 100.3{ }^{\circ} \mathrm{C}, 99.3{ }^{\circ} \mathrm{C}$ and $100.1{ }^{\circ} \mathrm{C}$, respectively. The $\mathrm{T}_{\mathrm{g}}$ values of the PMMA composites change very little in comparison with the neat PMMA. Although a slight increase of the mobility of polymer chains upon introducing the Pickering surfactant particles has been reported ${ }^{24}$, we could not see a trend in our data beyond the experimental error.

\subsubsection{Mechanical Properties of PMMA/CRC Composites}

The mechanical properties of the PMMA and the PMMA/CRC composites were determined and compared with that of neat PMMA to examine the influence of CRC on the deformation behavior of the nanocomposites. The results are shown in Figure 4.5. The flexural modulus increased compared to that of neat PMMA for all composites with different $\mathrm{CRC}$ contents, which indicated that $\mathrm{CRC}$ acted as reinforcement in the PMMA matrix by transferring load from the PMMA to the $\mathrm{CRC}^{25}$. In the Figure 4.5(b), PMMA-1\%-CRC has the highest flexural modulus of $80.5 \mathrm{MPa}$, enhancements of $10.0 \%$ compared with neat PMMA. Interestingly, flexural strength values do not increase significantly with increasing CRC content within the experimental error, probably due to the conflicting effect of filler content and the association of $\mathrm{CRC}^{26}$. This might be attributed to the network formation of CRC in the PMMA matrix following compression ${ }^{27}$. 

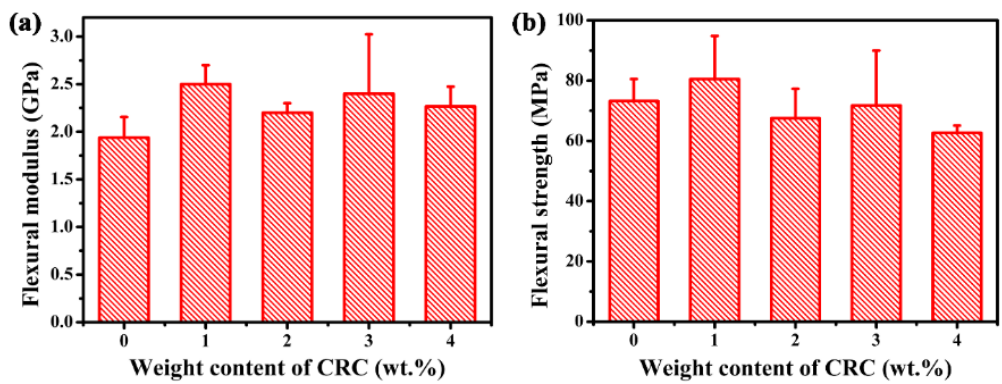

Figure 4.5 (a) flexural modulus and (b) strength of neat PMMA and PMMA/CRC composites.

\subsubsection{Differently Colored PMMA/CRC Composites}

As is shown in Figure 4.6, PMMA/CRC composites with different color can be prepared by using RC dyed with blue, red and yellow dyes as the Pickering stabilizer. According to our previous research, any desirable color shade could be produced by blending CRCs of the three prime colors, namely red, blue, and yellow. Therefore, the procedure reported herein is generic to making colored PMMA/RC composites of any color.

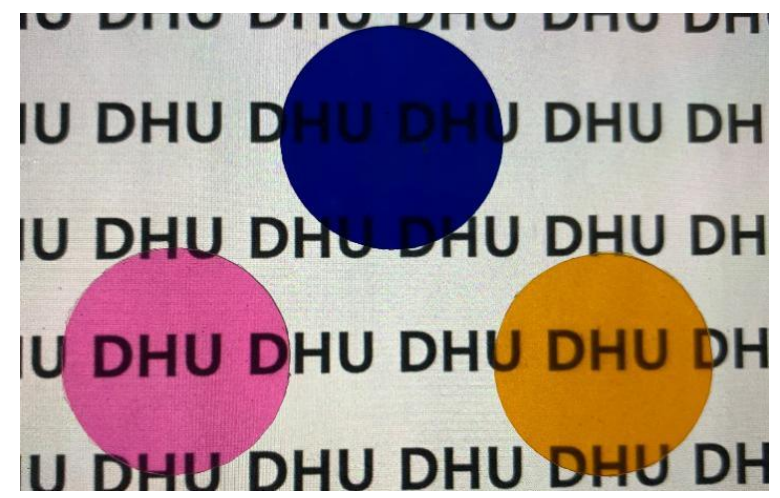

Figure 4.6 Optical image of colored PMMA/CRC composites with different color.

\subsection{Conclusions}

Colored polymer/CRC composites prepared via Pickering emulsion approach are reported with PMMA as matrix material chosen to demonstrate the approach. Color was achieved by grafting dye molecules to cellulose. The colored Pickering emulsion containing oily PMMA phase could be efficiently stabilized by CRC. Composite microspheres were prepared by removing the solvent DCM and water. The color of the composites is uniform in the PMMA matrix and no CRC aggregate is observed. 
The color depth of the composites could be tuned via adjusting the CRC contents in the PMMA matrix. An increase of the thermal stability was observed for the nanocomposites when compared with the neat polymer. The flexural modulus of PMMA composites was enhanced for all CRC contents. The Pickering emulsion procedure used in this work can be applied to obtain composites exhibiting other colors, reinforced by the environmental friendly CRC, as well as to other polymers, thus providing a generic platform.

\subsection{Experimental sections}

\subsubsection{Materials}

Polymethyl methacrylate (PMMA) with a number-average molecular weight $\left(M_{n}\right)$ of $\sim 46000 \mathrm{Da}$ and weight-average $(\mathrm{Mw})$ of $\sim 110,000 \mathrm{Da}$, was supplied by Energy Chemical, China.

Wood Pulp was obtained from the Xinxiang Natural Chemical Co., Ltd. The viscosity-average degree of polymerization measured in a cupric ethylene diamine hydroxide (CUEN) solution was 870. Solution viscosity measurements were performed using an Ubbelohde viscometer.

Colour Index (C.I.) Reactive Red 24 (RR24) pigments were supplied by Taoyuan Dyestuff Co., Ltd, Wujiang, China. Phosphoric acid (85\%) was received from Titan Scientific Co., Ltd, Shanghai, China. Sodium chloride $(\mathrm{NaCl})$, sodium carbonate $\left(\mathrm{Na}_{2} \mathrm{CO}_{3}\right)$, sodium hydroxide $(\mathrm{NaOH})$ and Dichloromethane $\left(\mathrm{CH}_{2} \mathrm{Cl}_{2}\right)$ were supplied by Sinopharm Chemical Reagent CO., Ltd. All chemicals were used as received.

\subsubsection{Preparation of $R C$ Suspensions}

RC suspensions were prepared by a previously reported procedure ${ }^{14}$. In short, phosphoric acid and deionized water were pre-cooled to $4{ }^{\circ} \mathrm{C}$. Wood pulp (2 g) was pre-wetted with $6 \mathrm{ml}$ deionized water and $200 \mathrm{ml} \mathrm{85 \%}$ phosphoric acid was added thereto. The mixture was stirred at $5{ }^{\circ} \mathrm{C}$ for $24 \mathrm{~h}$ to obtain a clear solution. Subsequently, $1 \mathrm{~L}$ of deionized water was used to regenerate the cellulose. The dispersion was centrifuged at $12000 \mathrm{rpm}$ (Thermo, USA) until a constant $\mathrm{pH}$ was reached. Prior to use, the $\mathrm{RC}$ was re-dispersed twice at 800 bar using a high-pressure homogenizer (APV-2000 Homogenizer, Germany).

\subsubsection{Preparation of CRC Suspensions}

The CRC suspension was prepared by a method previously reported by our group ${ }^{4}$. 
RC suspension and RR24 dye were added into a three-necked flask at room temperature. A predetermined amount of aqueous $\mathrm{NaCl}(20 \mathrm{~g} / \mathrm{L})$ was added after 15 min. Following this step, the mixture was heated to and maintained at $70{ }^{\circ} \mathrm{C} . \mathrm{Na}_{2} \mathrm{CO}_{3}$ $(15 \mathrm{~g} / \mathrm{L})$ was added into the mixture $30 \mathrm{~min}$ later. The mixture was allowed to react for $1 \mathrm{~h}$. Finally, the dye bath was subsequently cooled to room temperature and the CRC was washed with D.I. water by centrifugation until the supernatant became colorless.

\subsubsection{Preparation of PMMA/CRC Composites}

A CRC dispersion was diluted with deionized water to obtain cellulose dispersions of concentrations at $0.05,0.1,0.15$ and $0.2 \% \mathrm{w} / \mathrm{v}$. The CRC to PMMA ratio was adjusted (1, 2, 3 and $4 \mathrm{wt} . \%)$ to deliver the targeted CRC composition of the composites. A solution of PMMA in $\mathrm{CH}_{2} \mathrm{Cl}_{2}(10 \mathrm{ml}, 100 \mathrm{mg} / \mathrm{ml})$ was added to the CRC aqueous dispersion $(50 \mathrm{ml})$. The mixture was homogenized (IKA T18 homogenizer, Germany) at $12,000 \mathrm{rpm}$ for $3 \mathrm{~min}$ followed by ultrasonication (SCIENTZ, JY 92-IIDN, China) for 3 min to obtain Pickering emulsions. $\mathrm{CH}_{2} \mathrm{Cl}_{2}$ was evaporated at ambient temperature over the course of $24 \mathrm{~h}$. The precipitates were vacuum filtered using a 500-mesh filter screen. The paste collected on the filter screen was vacuum dried at $60^{\circ} \mathrm{C}$ for $24 \mathrm{~h}$. The PMMA/CRC composite films were prepared by hot-pressing of the dried paste employing a Carver Laboratory Press (Carver Inc., USA) at $170{ }^{\circ} \mathrm{C}$ and $1500 \mathrm{Kg}$ for $5 \mathrm{~min}$.

\subsubsection{Characterization}

The emulsion obtained was observed using an optical microscope (Eclipse E100, Nikon, Japan). After filtration, PMMA/CRC composite microspheres were observed with scanning electron microscopy (SEM, TM-1000, Hitchi, Japan) at $2 \mathrm{kV}$ and 6-8 A. The samples were dried at $40^{\circ} \mathrm{C}$ for overnight. Then they were coated with a goldpalladium alloy.

Transmittance UV-VIS spectra of the neat PMMA and PMMA/CRC composite films were obtained using an optical spectrophotometer (UV-3600, Shimadzu).

Thermal properties of neat PMMA and PMMA/CRC composites were characterized by differential scanning calorimetry (DSC, 214, Netzsch, Germany) equipped with a cooling system, under a nitrogen atmosphere. The samples (5-10 mg) were loaded and sealed in aluminum pans. The samples were heated from 25 to $200{ }^{\circ} \mathrm{C}$ at a ramp speed of $10{ }^{\circ} \mathrm{C} / \mathrm{min}$, isothermed for $5 \mathrm{~min}$ to eliminate thermal history, cooled to $25^{\circ} \mathrm{C}$ at $2{ }^{\circ} \mathrm{C} / \mathrm{min}$, and heated again to $200{ }^{\circ} \mathrm{C}$ at $10{ }^{\circ} \mathrm{C} / \mathrm{min}$ under nitrogen 
purge. The glass transition temperature $\left(\mathrm{T}_{\mathrm{g}}\right)$ was determined from the second heating scan.

The thermal decomposition of neat PMMA and PMMA/CRC composites were characterized to determine their thermal stability using thermogravimetric analysis (TG, Netzsch 209F3, Germany). The specimens were scanned from $30{ }^{\circ} \mathrm{C}$ to $600{ }^{\circ} \mathrm{C}$ at the rate of $10^{\circ} \mathrm{C} / \mathrm{min}$ and analyses were performed under a nitrogen gas flow.

Flexural testing was conducted using a universal testing machine (UH6502, Youhong, China) on rectangular specimens $\left(50.8 \times 12.7 \times 0.5 \mathrm{~mm}^{3}\right)$ at a support span of $25.4 \mathrm{~mm}$ and a crosshead speed of $5 \mathrm{~mm} / \mathrm{min}$ according to the procedure described in ASTM D 790-2007. 


\section{Appendix}
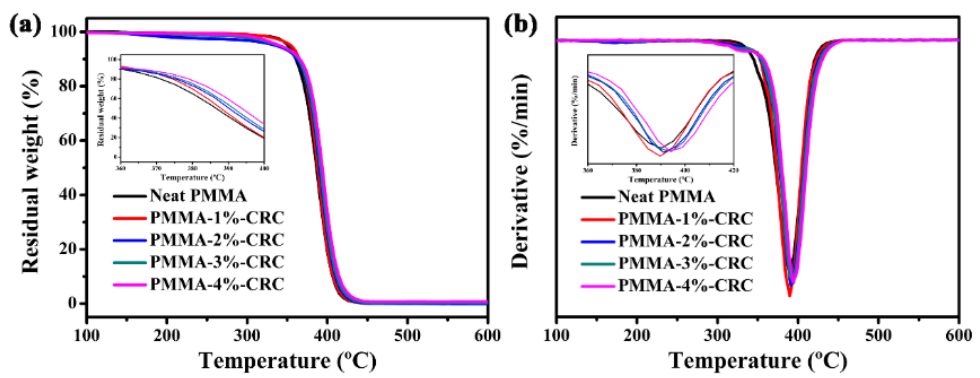

Figure 4-S1 (a) TG and (b) DTG thermograms of neat PMMA and PMMA/CRC composites.

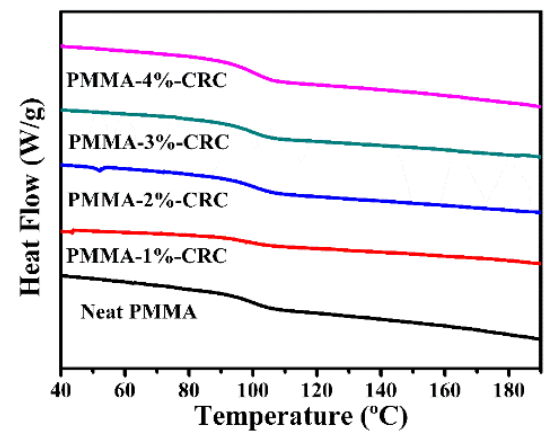

Figure 4-S2 DSC traces during cooling scan of neat PMMA and PMMA/CRC composites. 


\section{References}

[1] Pfaff, G.; Franz, K.; Emmert, R.; Nitta, K., Ullmann's Encyclopedia of Industrial Chemistry: Pigments, Inorganic, see Section 4.3, 6th edn.(electronic release). VCH Verlagsgesellschaft, Weinheim: 1998.

[2] Maile, F. J.; Pfaff, G.; Reynders, P. Effect pigments-past, present and future. Progress in Organic Coatings 2005, 54 (3), 150-163.

[3] Landfester, K. Miniemulsion polymerization and the structure of polymer and hybrid nanoparticles. Angewandte Chemie International Edition 2009, 48 (25), 4488-4507.

[4] Ding, L.; Jiang, Y.; Wang, B.; Li, Y.; Mao, Z.; Xu, H.; Zhong, Y.; Zhang, L.; Sui, $\mathrm{X}$. A waterborne bio-based polymer pigment: colored regenerated cellulose suspension from waste cotton fabrics. Cellulose 2018, 25 (12), 7369-7379.

[5] Zhao, J.; Ding, L.; Sui, X.; Mao, Z.; Xu, H.; Zhong, Y.; Zhang, L.; Chen, Z.; Wang, B. Bio-based polymer colorants from nonaqueous reactive dyeing of regenerated cellulose for plastics and textiles. Carbohydrate Polymers 2019, 206, 734-741.

[6] Sahoo, P. K.; Samal, R. Fire retardancy and biodegradability of poly (methyl methacrylate)/montmorillonite nanocomposite. Polymer Degradation and Stability 2007, 92 (9), 1700-1707.

[7] Agrawal, M.; Gupta, S.; Zafeiropoulos, N. E.; Oertel, U.; Häßler, R.; Stamm, M. Nano-level mixing of $\mathrm{ZnO}$ into poly (methyl methacrylate). Macromolecular Chemistry and Physics 2010, 211 (17), 1925-1932.

[8] Ali, U.; Karim, K. J. B. A.; Buang, N. A. A review of the properties and applications of poly (methyl methacrylate)(PMMA). Polymer Reviews 2015, 55 (4), 678-705.

[9] Klemm, D.; Kramer, F.; Moritz, S.; Lindström, T.; Ankerfors, M.; Gray, D.; Dorris, A. Nanocelluloses: a new family of nature-based materials. Angewandte Chemie International Edition 2011, 50 (24), 5438-5466.

[10] Dufresne, A. Nanocellulose: a new ageless bionanomaterial. Materials Today 2013, $16(6), 220-227$.

[11] Banerjee, M.; Sain, S.; Mukhopadhyay, A.; Sengupta, S.; Kar, T.; Ray, D. Surface treatment of cellulose fibers with methylmethacrylate for enhanced properties of in situ polymerized PMMA/cellulose composites. Journal of Applied Polymer 
Science 2014, 131 (2), 39808.

[12] Xu, W.; Qin, Z.; Yu, H.; Liu, Y.; Liu, N.; Zhou, Z.; Chen, L. Cellulose nanocrystals as organic nanofillers for transparent polycarbonate films. Journal of Nanoparticle Research 2013, 15 (4), 1562.

[13] Zhang, Y.; Wu, J.; Wang, B.; Sui, X.; Zhong, Y.; Zhang, L.; Mao, Z.; Xu, H. Cellulose nanofibril-reinforced biodegradable polymer composites obtained via a Pickering emulsion approach. Cellulose 2017, 24 (8), 3313-3322.

[14] Zhang, Y.; Jiang, Y.; Han, L.; Wang, B.; Xu, H.; Zhong, Y.; Zhang, L.; Mao, Z.; Sui, X. Biodegradable regenerated cellulose-dispersed composites with improved properties via a Pickering emulsion process. Carbohydrate Polymers 2018, 179, 86-92.

[15] Li, X.; Hegyesi, N.; Zhang, Y.; Mao, Z.; Feng, X.; Wang, B.; Pukánszky, B.; Sui, $\mathrm{X}$. Poly (lactic acid)/lignin blends prepared with the Pickering emulsion template method. European Polymer Journal 2019, 110, 378-384.

[16] Du, C.; Wang, C.; Tian, A.; Xu, C. Properties of lyocell spinning solution with the addition of carbon black/latex composite. Colloids and Surfaces A: Physicochemical and Engineering Aspects 2013, 428, 1-8.

[17] Li, X.; Ding, L.; Zhang, Y.; Wang, B.; Jiang, Y.; Feng, X.; Mao, Z.; Sui, X. Oilin-water Pickering emulsions from three plant-derived regenerated celluloses. Carbohydrate Polymers 2019, 207, 755-763.

[18] Xuejuan, J.; Yingwen, C.; Chong, S.; Yangfan, Y.; Peng, W.; Xiaoxiong, Z.; Tao, W. Preparation and characterization of cellulose regenerated from phosphoric acid. Journal of Agricultural and Food Chemistry 2013, 61 (50), 12405-12414.

[19] Littunen, K.; Hippi, U.; Saarinen, T.; Seppälä, J. Network formation of nanofibrillated cellulose in solution blended poly(methyl methacrylate) composites. Carbohydrate Polymers 2013, 91 (1), 183-190.

[20] Chen, L. S.; Huang, Z. M.; Dong, G. H.; He, C. L.; Ling, L.; Hu, Y. Y.; Yan, L. Development of a transparent PMMA composite reinforced with nanofibers. Polymer Composites 2010, 30 (3), 239-247.

[21] Perinović, S.; Andričić, B.; Erceg, M. Thermal properties of poly( 1 lactide)/olive stone flour composites. Thermochimica Acta 2010, 510 (1), 97-102.

[22] Pielichowski, K.; Njuguna, J. Thermal Degradation of Polymeric Materials, 2005; p 10. 
[23] Santmarti, A.; Teh, J. W.; Lee, K.-Y. Transparent poly (methyl methacrylate) composites based on bacterial cellulose nanofiber networks with improved fracture resistance and impact strength. ACS Omega 2019, 4 (6), 9896-9903.

[24] Chen, L. S.; Huang, Z. M.; Dong, G. H.; He, C. L.; Liu, L.; Hu, Y. Y.; Li, Y. Development of a transparent PMMA composite reinforced with nanofibers. Polymer Composites 2009, 30 (3), 239-247.

[25] Kiziltas, E. E.; Kiziltas, A.; Bollin, S. C.; Gardner, D. J. Preparation and characterization of transparent PMMA-cellulose-based nanocomposites. Carbohydrate Polymers 2015, 127, 381-389.

[26] Zhang, Y.; Cui, L.; Xu, H.; Feng, X.; Wang, B.; Pukánszky, B.; Mao, Z.; Sui, X. Poly (lactic acid)/cellulose nanocrystal composites via the Pickering emulsion approach: Rheological, thermal and mechanical properties. International Journal of Biological Macromolecules 2019, 137, 197-204.

[27] Dasan, Y.; Bhat, A.; Ahmad, F. Polymer blend of PLA/PHBV based bionanocomposites reinforced with nanocrystalline cellulose for potential application as packaging material. Carbohydrate Polymers 2017, 157, 1323 1332. 


\section{Chapter 5}

\section{Enzymatic Degradation of PLA/Cellulose Nanocrystal Composites}

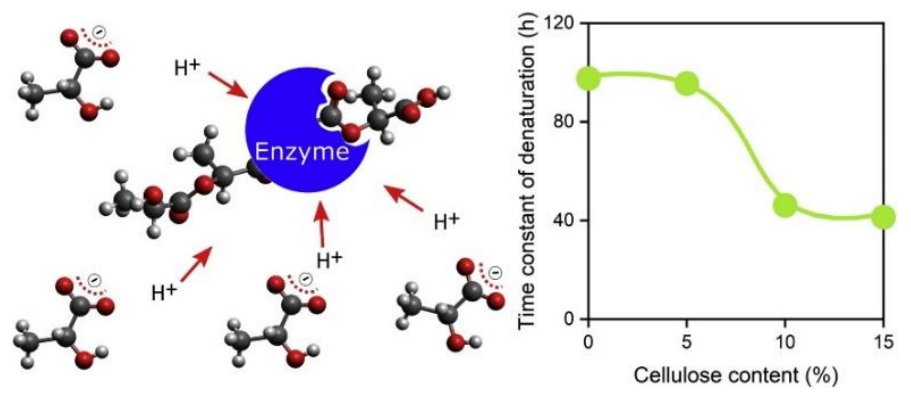

The enzymatic degradation of poly (lactic acid) (PLA) and its nanocomposites reinforced with cellulose nanocrystals (CNC) was catalyzed with lipase from Candida rugosa and proteinase $\mathrm{K}$ from Tritirachium album. The composites were prepared with the Pickering emulsion process and they contained 5, 10 and $15 \mathrm{wt} . \%$ nanocellulose. Compression molded plates were cut to pieces for the degradation experiments. Preliminary experiments showed that the lipase does not catalyze the degradation of PLA, but the proteinase $\mathrm{K}$ is very efficient. The lactic acid forming 
during the reaction decreases the $\mathrm{pH}$ of the degradation medium almost to 4 that leads to the denaturation of the enzyme. Besides $\mathrm{pH}$, the ion concentration of the solution also influences the rate of degradation; smaller ionic strength is more advantageous. The cellulose nanocrystals used for the reinforcement of PLA increase the rate of degradation and the samples disintegrate very rapidly, the polymer degrades in three days. Because the samples lose their integrity, also the amount of lactic acid forming in the process was determined with a colorimetric assay with iron (III) chloride hexahydrate to follow degradation. A model was applied for the quantitative analysis of the kinetics of degradation and denaturation. The rate of both processes doubles in the presence of cellulose nanocrystals. The model and the obtained parameters can be used for the design of experiments and the prediction of the enzymatic degradation of aliphatic polyesters as well as their blends and composites.

\footnotetext{
*The contents of this chapter have been published as: Nóra Hegyesi, Yunchong Zhang, Andrea Kohári, Péter Polyák, Xiaofeng Sui, Béla Pukánszky; Enzymatic Degradation of PLA/Cellulose Nanocrystal Composites; Industrial Crops and Products, 2019, 141, 111799.
} 


\subsection{Introduction}

Biopolymers are used in increasing amounts in all areas of the economy ${ }^{1-3}$. The driving force of the application of these materials is the increased environmental awareness of the industry and the public, depleting resources of fossil energy, increasing difficulties of handling plastic waste, dangerous contamination of natural waters by microplastics, etc ${ }^{4-5}$. Biopolymers can be natural polymers like cellulose, starch and lignin or they can be based on natural resources like bio PE or polyamides and also poly(lactic acid) (PLA) ${ }^{3,6}$. Because of its advantageous properties, PLA is used in increasing quantities mostly for packaging and in biomedical applications ${ }^{7}$. However, PLA has some drawbacks as well, it is very sensitive to water during processing, brittle, crystallizes very slowly, but its physical ageing is relatively fast, which further decreases its fracture resistance. Many attempts are made to overcome these deficiencies by modification.

Various approaches are used to improve the processability and physical properties of PLA. Plasticization and blending improve deformability and impact resistance ${ }^{8-9}$, while the use of fillers and reinforcements increases stiffness ${ }^{10-11}$. In order to conserve one of the advantageous properties of PLA, biodegradability, it is often modified with lignocellulosic fibers. The addition of wood and cellulose fibers to PLA increases its stiffness and strength indeed, but decreases deformability even further. An obvious way to overcome this problem is to use cellulosic nanofillers for reinforcement ${ }^{12}$. Microfibrillar or bacterial cellulose and cellulose nanocrystals can be added to PLA in small amounts, still result in considerable improvement in properties. The stiffness and strength of the polymer increases, amorphous PLA maintains its transparency and even the tendency towards physical ageing may decrease as a result ${ }^{13}$. In addition, these fillers are also biodegradable ${ }^{14-15}$.

PLA is stable under ambient conditions thus its composites reinforced with cellulosic fillers can be used also in structural applications. On the other hand, it is compostable and can be degraded under biological conditions. Composting is a viable way to handle bioplastic waste and produce valuable material as a result. Consequently, the degradation of biopolymers is of theoretical and practical interest, together with the effect of lignocellulosic reinforcements on the degradation process. The degradation of PLA in compost has been studied intensively ${ }^{16-17}$, for example, investigated the effect of crystalline nanocellulose $(\mathrm{CNC})$ on the compostability of PLA and found that although PLA degraded under the conditions of the compost, the nanocellulose filler did not influence degradation practically at all. 
More attempts were made to study the enzymatic degradation of PLA. Enzymes are produced by microorganisms and the same organisms facilitate the degradation of biopolymers in the compost. A wide variety of enzymes was used to accelerate the degradation of PLA including kutinases, lipases, proteases and esterases ${ }^{18-22}$. The various enzymes catalyzed the degradation of PLA in different extents depending on the conditions of degradation. Certain lipases are advantageous because of their larger resistance against environmental effects including solvents ${ }^{23-24}$. Although quite a few of the enzymes catalyzed the degradation of PLA in smaller or larger extent, proteinase seemed to be the most efficient among the enzymes used up to now.

The effect of lignocellulosic reinforcements on the enzymatic degradation of PLA was also investigated in a few cases. Stepczynska and Rytlewski ${ }^{25}$ studied the enzymatic degradation of PLA reinforced with flax and found that weight decrease is larger in its presence than without it and explained the phenomenon with the formation of channels due to the presence of the fibers. On the other hand, Luzi et al. ${ }^{17}$ found that the degradation of PLA slowed down considerably in the presence of crystalline nanocellulose already at $1 \mathrm{wt} . \%$ filler content. They also followed degradation by the determination of the weight loss of the samples.

Because of the importance of the biodegradation of PLA reinforced with cellulose nanocrystal fillers and due to the contradictory nature of the conclusions published, the goal of this work was to study the enzymatic degradation of PLA modified with crystalline nanocellulose. Two different enzymes were employed and degradation was followed not only by the measurement of weight, but also by an analytical technique. The influence of various factors on the enzymatic degradation of PLA was studied including the effect of the degradation product and ion concentration. The kinetics of degradation was analyzed quantitatively with the help of an appropriate model. Importance for practice is also mentioned in the final section of the paper.

\subsection{Results and Discussion}

The results are discussed in several sections. Those of the preliminary experiments directed towards the selection of the proper enzyme are presented first, and then the effect of experimental conditions on the extent and rate of degradation are shown next. The degradation of the nanocomposites and the effect of the nanofiller on it are discussed in the subsequent section followed by the quantitative analysis of degradation kinetics. 


\subsubsection{Preliminary experiments}

The goal of the preliminary experiments was to select the enzyme used in the further course of the study. Based on literature information, we selected the lipase from Candida rugosa (CRL) and a protease (proteinase $\mathrm{K}$ from Tritirachium album). Lipases are very robust and are not sensitive to environmental conditions ${ }^{23-24}$, and several reports indicated their smaller or larger efficiency in the degradation of various aliphatic polyesters ${ }^{26-30}$. On the other hand, quite a number of studies indicated that proteinase $\mathrm{K}$ degrades PLA efficiently ${ }^{17,21,25,31}$. The relative weight loss observed in the presence of CRL is plotted in Figure 5.1 as a function of time. The total weight loss at equilibrium is around $1.5 \%$ and the decrease of weight measured in the buffer not containing the enzyme is only slightly smaller. The presence of lactic acid was checked in the degradation solution by the analytical technique described in the experimental part and we could not detect any monomer in the solution at all. We had to draw the conclusion from these results that CRL does not catalyze the degradation of our PLA.

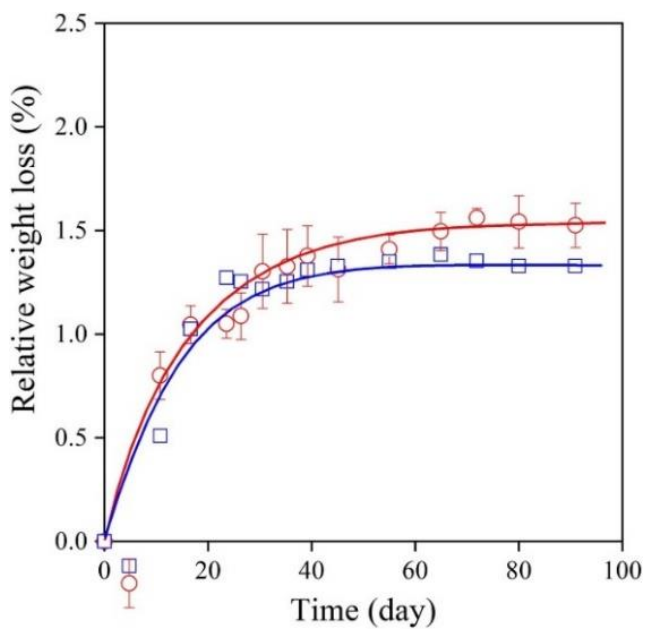

Figure 5.1 Weight loss of neat PLA in the presence of CRL. Symbols: (०) with enzyme, $(\square)$ buffer $(\mathrm{pH}=7.2)$.

Similar experiments were carried out with proteinase K as well. The samples lost much more weight quite fast, but degradation stopped in a relatively short time. Degradation restarted after changing the degradation medium, but it stopped again very soon (Figure 5.2). The measurement of the $\mathrm{pH}$ of the solution showed that $\mathrm{pH}$ changed from the original value of 8.6 to around 4.3-4.5. According to the producer 
the optimum $\mathrm{pH}$ range of this enzyme is between 6.5 and 12 , thus we must assume that the drastic decrease of $\mathrm{pH}$ resulted in the denaturation of the enzyme. We must call the attention here also to the weight measured in the buffer itself. Weight decreased only about $1.3 \%$ at $\mathrm{pH} 7.2$, while weight loss was approximately $5 \%$ at 8.6 . Probably the hydroxide ions present at $\mathrm{pH} 8.6$ catalyzed the hydrolytic degradation of PLA in accordance with the findings of Tsuji and Ikarashi ${ }^{32}$. According to the results of the preliminary study, we decided to conduct further experiments with proteinase $\mathrm{K}$, but the results also called attention to the role of the degradation product and the importance of $\mathrm{pH}$ during degradation.

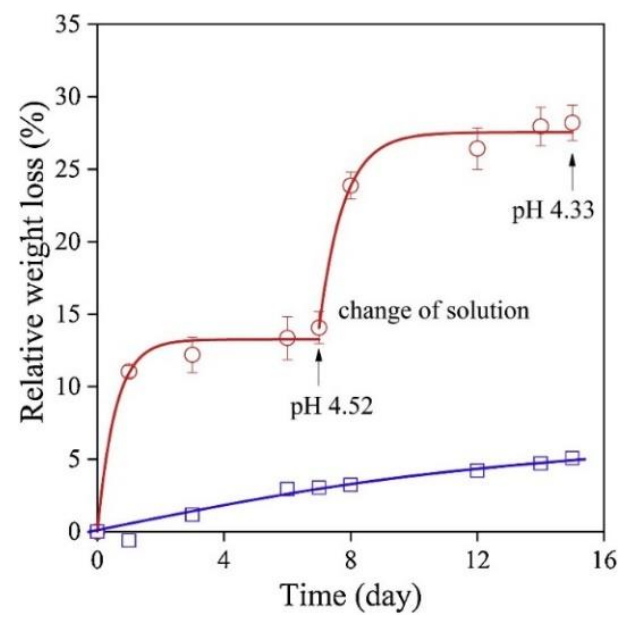

Figure 5.2 Catalytic action of proteinase $\mathrm{K}$ in the degradation of neat PLA. Effect of $\mathrm{pH}$ and the changing of the degradation medium. Symbols: $(\circ)$ with enzyme, $(\square)$ buffer $(\mathrm{pH}=8.6$, $\mathrm{I}=12 \mathrm{mM})$.

\subsubsection{Effect of $p H$}

The preliminary experiment carried out with proteinase $\mathrm{K}$ indicated that the $\mathrm{pH}$ of the degradation medium changes with time and degradation stops, probably because of the denaturation of the enzyme. Consequently, the change of $\mathrm{pH}$ was followed as a function of time in another experiment. According to Figure 5.3, pH decreases continuously indeed, practically linearly at the beginning and then slowing down at longer times. Weight loss is plotted in the figure as well for comparison. The two correlations correspond to each other perfectly, weight loss increases proportionally with time first and then it slows down and stops at longer times. Obviously, the 
formation of the degradation product, lactic acid $(\mathrm{pKa}=3.86)$, changes $\mathrm{pH}$ and stops the degradation of the polymer.

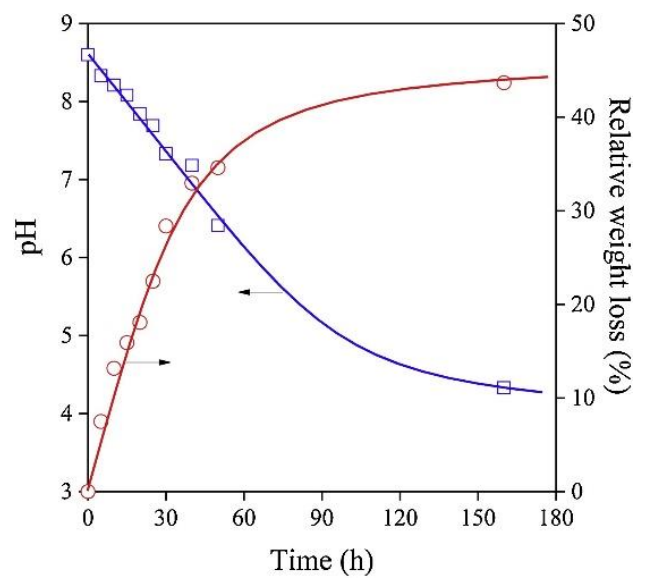

Figure 5.3 Effect of $\mathrm{pH}$ on the degradation (weight loss) of neat PLA. Decreasing rate below $\mathrm{pH}=6.5$. Symbols: (०) weight loss, $(\square) \mathrm{pH}$. (initial $\mathrm{pH}=8.6, \mathrm{I}=12 \mathrm{mM}$ ).

The analysis of the data showed that degradation slows down at around $33 \%$ weight loss resulting in $27 \mathrm{mmol} / 1$ lactic acid concentration. At this concentration, the buffer capacity of the solution is exhausted, $\mathrm{pH}$ decreases below a critical value, around 6.5 , at which the enzyme is not active any more. The denaturation of the enzyme leads to the slow down and stopping of degradation. This observation is in close agreement with that of Ghorpade et al. ${ }^{16}$ who found that a compost could not contain more than $30 \mathrm{wt} . \%$ PLA without losing its capacity to degrade the polymer. They explained the phenomenon with the formation of excessive lactic acid, which decreased $\mathrm{pH}$ and destroyed microorganisms. The results presented in this section showed that further experiments must be carried out in buffers with larger capacity.

\subsubsection{Ion concentration}

The effect of the ionic strength of the degradation medium was investigated in a further experiment. The measurements were carried out with the neat PLA, but also with the nanocomposites. The effect of ion concentration on the weight loss of the neat polymer is presented in Figure 5.4. Weight did not change in the buffer at all, but considerable weight loss was measured in the two solutions with different ionic strengths. With increasing ion concentration, the weight loss reached in equilibrium 
decreased considerably indicating that larger ion concentration decreases the activity of the enzyme. As a result of these measurements, the decision was made to continue experiments with a buffer of smaller ionic strength.

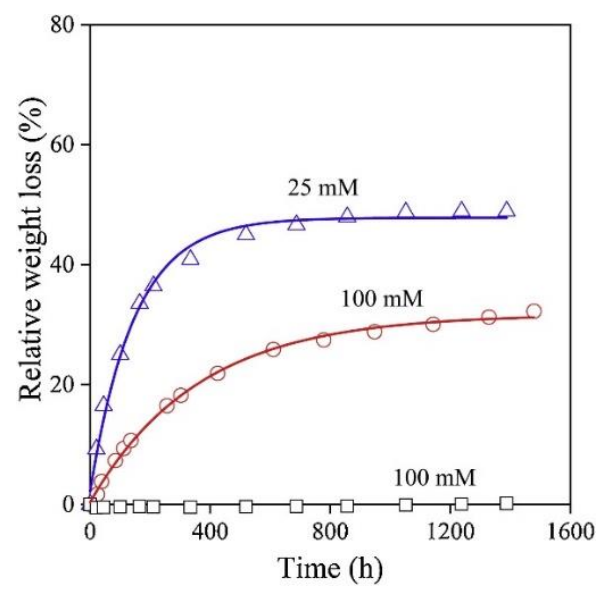

Figure 5.4 Influence of the ionic strength of the buffer solution on the enzymatic activity of proteinase K. Symbols: $(\triangle$ ) enzyme, $I=25 \mathrm{mM}$, (०) enzyme, $I=100 \mathrm{mM}$, ( $\square$ ) buffer, $\mathrm{I}=100 \mathrm{mM}(\mathrm{pH}=8.6)$.

\subsubsection{Enzymatic degradation}

Degradation was followed in different ways. Changes in the morphology and structure of the specimens gave some qualitative information about degradation, while the measurement of weight loss and the analytical method used yielded quantitative results.

\subsubsection{Structure}

Photos were taken of the specimens after sampling to follow changes in morphology. They are presented in Figure 5.5. The neat PLA became opaque after a day, then its smooth edges changed to sharp and broken ones, later the pieces became thinner and holes appeared in them. Finally, also the shape of the originally rectangular specimens changed and became irregular. Nevertheless, the specimens could be handled, weighed and photographed for a relatively long time. PLA containing the nanocellulose reinforcement, on the other hand, behaved completely differently. The samples fell apart very fast and disintegrated within a few days. This made impossible the measurement of their weight, thus the following of degradation in the usual way. 


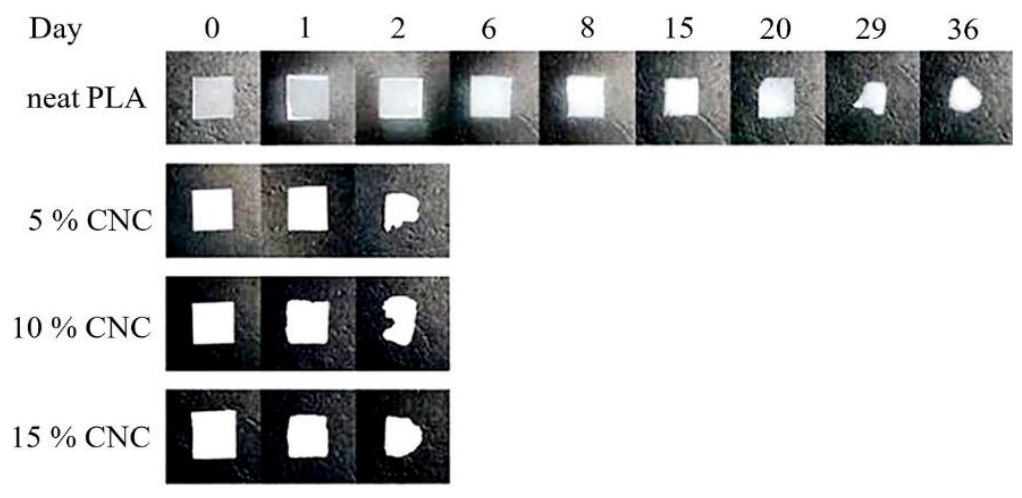

Figure 5.5 Changes in the appearance of neat PLA and disintegration of the PLA/CNC composites during their enzymatic degradation $(\mathrm{pH}=8.6, \mathrm{I}=25 \mathrm{mM})$.

Further information is offered about the structure of the composites by XRD measurements. Traces of PLA and its composites before and after $48 \mathrm{~h}$ degradation are presented in Figure 5.6. The broad peak between 9 and $27^{\circ}$ of the neat PLA shows that the polymer is practically completely amorphous which also can be observed on the diffractograms of the composites. This fact explains the fast degradation, since crystalline PLA degrades much slower ${ }^{32}$. The XRD traces of the composites differ considerably from that of the neat PLA. The peaks characteristic for crystalline cellulose appear clearly in the trace and superimpose onto the amorphous halo of PLA (Figure 5.6a). The intensity of the reflections at 16.5 and $22.7^{\circ}$ increases with increasing nanocellulose content. These reflections become even more pronounced after degradation, and they dominate the diffractogram (Figure 5.6b). Obviously, PLA degrades and it is removed from the composite leaving behind the cellulose nanocrystals. All these results prove that enzyme solutions degrade PLA indeed and nanocellulose facilitates degradation. It also indicates that $\mathrm{CNC}$ remains intact during the process. 
a)

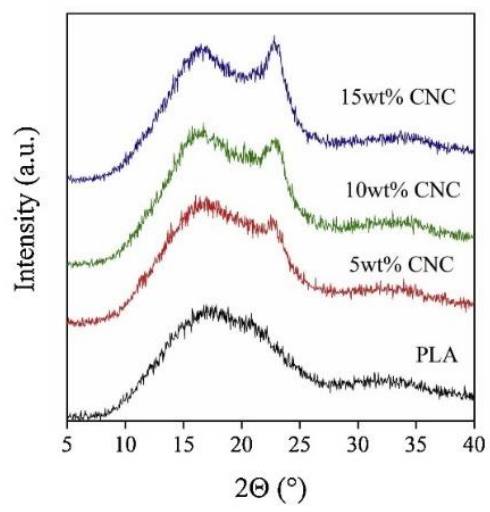

b)

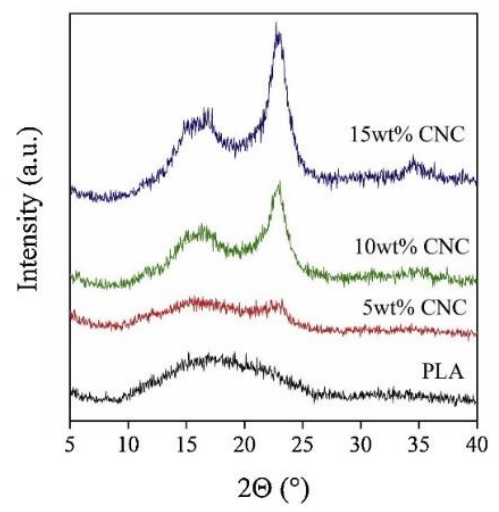

Figure 5.6 XRD traces of neat PLA and that of the CNC nanocomposites a) before the start of the degradation experiments and $\mathrm{b})$ after $48 \mathrm{~h}$ degradation $(\mathrm{pH}=8.6, \mathrm{I}=25 \mathrm{mM})$.

\subsubsection{Weight loss}

The enzymatic degradation of aliphatic polyesters is usually followed by the measurement of weight, the determination of weight loss. The results of such measurements are presented in Figure $\mathbf{5 . 7}$ for the neat PLA and the PLA/CNC composites. In accordance with the results shown in Figure 5.5, degradation could be followed with this method quite well in the case of the neat polymer, but not for the composites, since they disintegrated very rapidly within a few days. The correlation obtained for the neat polymer is in complete agreement with previous results, after a certain time degradation slows down and converges towards a constant value, which however is very close to $100 \%$. The few results obtained for the composites indicate that weight loss is inversely proportional to nanocellulose content, i.e. the weight loss measured became smaller with increasing $\mathrm{CNC}$ content. These results and those presented in the previous section completely justify the use of another method for the detection of the degradation of the polymer. 


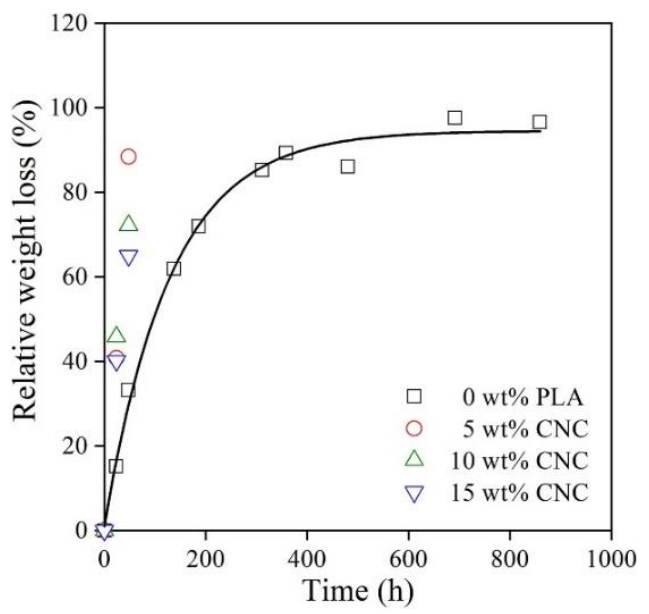

Figure 5.7 Relative weight loss of PLA samples measured during their enzymatic degradation in the presence of proteinase K. Symbols: $(\square)$ neat PLA, (०) 5, ( $\triangle) 10,(\vee) 15$ wt.\% crystalline nanocellulose $(\mathrm{pH}=8.6, \mathrm{I}=25 \mathrm{mM})$.

\subsubsection{Quantitative analysis of the degradation product}

Since composite samples disintegrated after a relatively short time, the usual technique of measuring weight loss could not be used to follow degradation. On the other hand, the determination of the amount of the degradation product, lactic acid, proved to be an adequate method for this purpose. The formed lactic acid could be recalculated into weight loss after calibration $\left(\varepsilon_{390 \mathrm{~nm}}=17.611 \mathrm{dm}^{3} \mathrm{~mol}^{-1} \mathrm{~cm}^{-1}\right)$, and this calculated weight loss is plotted against degradation time in Figure 5.8. Only two correlations are drawn in the figure to avoid confusion, but all the measured points are plotted. The results indicate that cellulose nanocrystals accelerate degradation compared to the neat polymer. However, large $\mathrm{CNC}$ content results in smaller degree of degradation, the reaction stops earlier than in the case of the neat polymer. The results clearly indicate that complexation using iron(III) chloride hexahydrate is an adequate method for the following of the enzymatic degradation of PLA. 


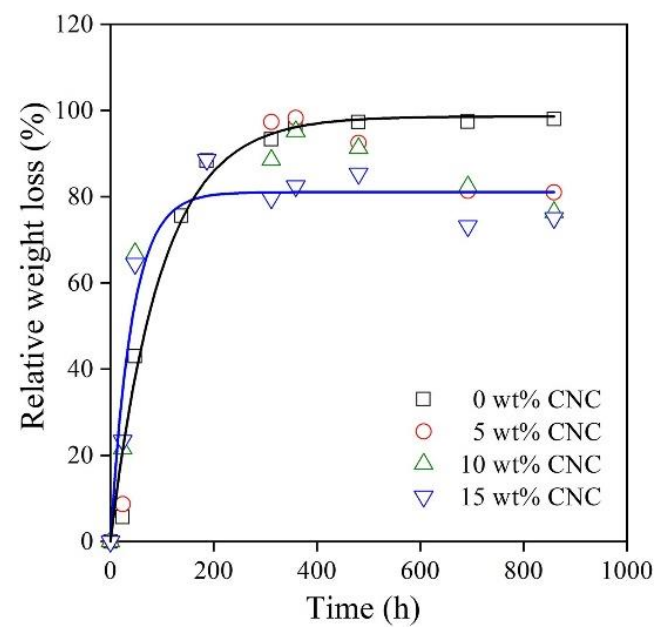

Figure 5.8 Relative weight loss calculated from the results of UV-vis measurements plotted against the time of enzymatic degradation. Symbols are the same as in Figure 5.7.

Because of the acidic character of the degradation product and the effect of $\mathrm{pH}$ on the degradation process, $\mathrm{pH}$ was monitored in all series of measurements done. The purpose of the determination was to see, if excessive denaturation of the enzyme occurred, or any other effect influenced $\mathrm{pH}$ and the rate as well as the extent of degradation. In Figure 5.9, the $\mathrm{pH}$ of the degradation medium is plotted against the amount of lactic acid formed in the experiments for the neat PLA and the three nanocomposites (see Figure 5.8). $\mathrm{pH}$ decreases slowly and almost proportionally up to a certain lactic acid concentration and then decreases very steeply. The sudden and steep change indicates that besides the formation of lactic acid another factor might also influence the kinetics of degradation. This factor is the loss of the capacity of the Tris buffer at this $\mathrm{pH}^{33}$, and thus the formation of a small amount of lactic acid leads to the sharp decrease of the $\mathrm{pH}$ of the solution. This calls attention again to the importance of choosing the proper medium for degradation. 


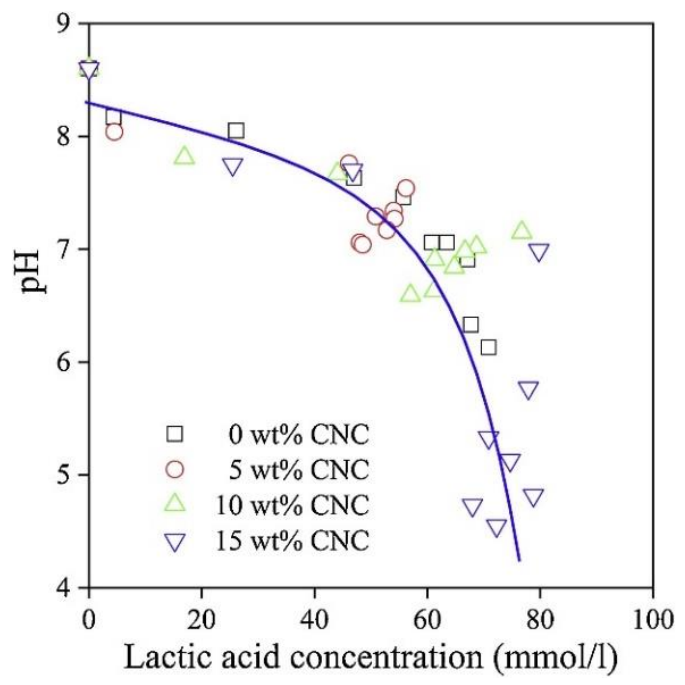

Figure 5.9 $\mathrm{pH}$ of the degradation solution plotted against its lactic acid concentration. Nonlinear correlation due to changing buffer capacity. Symbols are the same as in Figure 5.7.

\subsubsection{Degradation kinetics}

In order to analyze degradation properly and see the effect of cellulose nanocrystals on its rate, a model is needed which describes degradation kinetics quantitatively. The most frequently used model for the description of the kinetics of enzymatic reactions was proposed by Michaelis and Menten ${ }^{34}$. Unfortunately, the model was developed for homogeneous reactions, and usually it is used only for the description of experiments carried out for a short time, in the linear section of the reaction $^{35}$. In our case, the reaction is heterogeneous, and degradation is carried out for a long time, for days compared to several hundred minutes. Under such conditions, enzymatic degradation proceeds in three steps. The first step of the process is the adsorption of the enzyme on the surface of the substrate. This is followed by the degradation of the polymer, which may be described by the original Michaelis-Menten approach. Finally, after longer times, the denaturation of the enzyme may take place and the reaction slows down, just as in our case (see Figure 5.2, 5.4, 5.8 and 5.9).

The model, which describes the kinetics of enzymatic degradation quantitatively, must consider all three steps. However, since the adsorption of the enzyme on the substrate takes place first and in a short time compared to the entire length of the measurement, we ignore it here in order to simplify treatment. If we follow degradation by the loss of sample weight, the rate of degradation in the linear stage, 
as predicted by the Michaelis-Menten $\operatorname{model}^{34}$, can be described with the following differential equation

$$
\frac{d m(t)}{d t}=v_{d}
$$

where $v_{d}$ is the rate of degradation, $\mathrm{m}$ the weight loss of the sample and time. However, we must take into account the denaturation of the enzyme, which results in the decrease of degradation rate. If we assume that denaturation proceeds according to first order kinetics, the rate of mass loss can be expressed as

$$
\frac{d m(t)}{d t}=v_{d}-\frac{m(t)}{\tau}
$$

where $\tau$ is the time constant of denaturation, which expresses the rate of the loss of activity of the enzyme. After the necessary operations and integration, we obtain the following solution

$$
m(t)=\exp (-t / \tau)+v_{d} \tau
$$

where $C$ is an integration constant the value of which can be determined from the initial conditions of the setup. After defining $C$ and rearrangement, we come to the final solution

$$
m(t)=v_{d} \tau[1-\exp (-\mathrm{t} / \tau)]
$$

The three parameters of Eq. (4) all have real physical meaning. $v_{d}$ gives the initial rate of degradation at zero time, $\tau$ is a time constant which is related to the rate of denaturation, and the pre-exponential term $\mathrm{A}=v_{d} \tau$ equals the loss of mass at infinite time, i.e. the amount of polymer degraded by the enzyme. Naturally, the model can be used not only for the analysis of mass loss, but also any other quantity characterizing the degradation of aliphatic polyesters, i.e. the absorbance of the complex formed from the reaction of lactic acid and iron(III) chloride hexahydrate.

The model can be verified by fitting Eq. (4) to the experimental results. The solid lines in Figure 5.2, Figure 5.8 are fitted correlations and describe the results very well. Fitting the model to the experimental values allows the determination of the constants, which characterize the rate of degradation and denaturation. The calculations have been carried out for all the experiments done in the project as a function of time, but we present only the most important results here to support our conclusions. The parameters obtained for the two-step correlation of Figure 5.2 and those presented in Figure 5.8 are collected in Table 1. 
Table 1. Kinetic parameters of the degradation of PLA and its nanocomposites as well as the denaturation of the enzyme (see Eq. (1)).

\begin{tabular}{lcccc}
\hline Experiment & $\mathbf{C N C}$ content $(\mathbf{w t} . \%)$ & $\mathbf{V d}_{\mathbf{d}}(\boldsymbol{\%} / \mathbf{h})$ & $\boldsymbol{\tau}(\mathbf{h})$ & $\mathbf{A}(\boldsymbol{\%})$ \\
\hline preliminary & $\mathbf{0}$ & 0.51 & 32.7 & 16.8 \\
& $\mathbf{0}$ & 2.31 & 11.9 & 27.5 \\
degradation & $\mathbf{0}$ & 1.01 & 97.6 & 98.6 \\
& $\mathbf{5}$ & 0.95 & 95.5 & 90.7 \\
& $\mathbf{1 0}$ & 1.89 & 46.2 & 87.1 \\
& $\mathbf{1 5}$ & 1.96 & 41.4 & 81.1 \\
\hline
\end{tabular}

The results listed in Table 1 show that in spite of the fact that the composition of the initial degradation solution and that used in the second step was exactly the same, the degradation is much faster in the second stage than in the first. A possible reason for this increase might be the changing of the surface roughness of samples, which degraded to a certain extent. Since degradation proceeds on the surface, its rate increases as a result. Similarly, the rate of denaturation also increases, but this is probably the consequence of increased degradation rate. Faster degradation results in more lactic acid, faster change of $\mathrm{pH}$ and earlier denaturation. The parameters obtained for the neat PLA and the three composites are listed in the last four rows of Table 1. Apart from the composite containing $5 \mathrm{wt} \%$ nanocellulose, the rate of degradation increases considerably in the presence of the reinforcement (see Figure 5.10). Such an increase was observed by Stepczynska and Rytlewski ${ }^{25}$ and they explained it with the formation of some kind of channels, which conduct water into the polymer. However, degradation occurs only on the surface of the samples, since enzyme molecules are large and cannot diffuse into the polymer. We believe instead that degradation removes PLA leading to the disintegration of the samples and larger surface area, which allows the enzyme to adsorb on the surface and accelerate degradation. Besides the rate of degradation, also the time constant of denaturation is plotted in Figure 5.10 as a function of nanocellulose content. The rate of denaturation also increases, partly because of the faster rate of degradation (see above), but also because of the exhaustion of the buffer. Kinetic analysis verified our qualitative 
conclusions drawn from the results and expressed the effect of various factors quantitatively. The rate of denaturation has especially large importance, because it determines the lifetime of the degradation medium, the time until the enzyme used as catalyst is active.

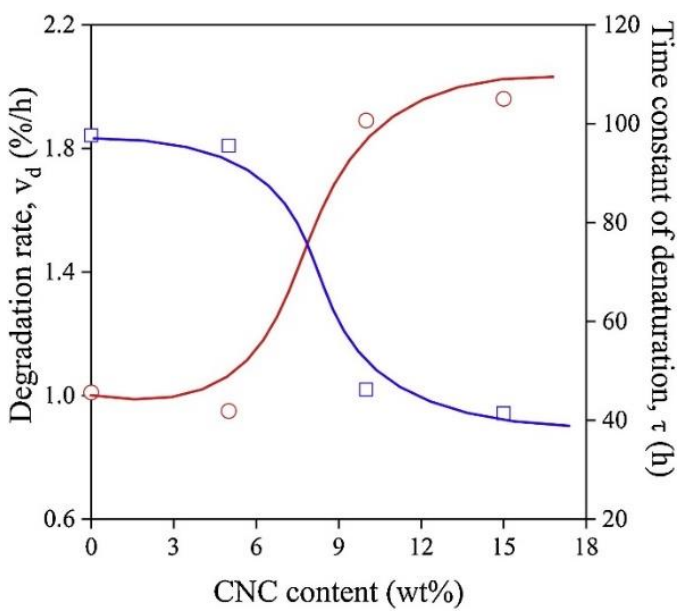

Figure 5.10 Effect of $\mathrm{CNC}$ content on the rate of degradation and the time constant of denaturation during the enzymatic degradation of PLA/CNC nanocomposites. Symbols: (०) rate of degradation, $v_{d} ;(\square)$ time constant of denaturation, $\tau$ (see Eq. 1).

\subsection{Conclusions}

Preliminary experiments showed that the lipase from Candida rugosa does not catalyze the degradation of PLA, but proteinase $\mathrm{K}$ is very efficient. The lactic acid forming during the reaction is a relatively strong acid, changes the $\mathrm{pH}$ of the degradation medium that leads to the denaturation of the enzyme. Denaturation occurs below $\mathrm{pH}=6.5$, thus either the degradation solution must be changed regularly or a buffer with sufficient capacity must be used as medium. Besides $\mathrm{pH}$, the ion concentration of the solution also influences the rate of degradation; smaller ionic strength is more advantageous. The cellulose nanocrystals used for the reinforcement of PLA increase the rate of degradation and the samples disintegrate very rapidly. Because the samples lose their integrity, an alternative method is needed to follow degradation. This can be done by the determination of the amount of lactic acid forming during degradation. Complex formation with iron(III) chloride hexahydrate and UV-vis spectroscopy proved to be a very appropriate method for the purpose. A 
model was applied for the quantitative analysis of the kinetics of degradation and denaturation, which takes into account and describes both processes well. The model and the obtained parameters can be used for the design of experiments and the prediction of the degradation of aliphatic polyesters as well as their blends and composites.

\subsection{Experimental sections}

\subsubsection{Materials}

The PLA used in the experiments was the Ingeo 2003D grade supplied by Natureworks, USA with a D-lactic acid content of $1.4 \%$, density of $1.24 \mathrm{~g} / \mathrm{cm}^{3}$, number-average molecular weight $(\mathrm{Mn})$ of $150,000 \mathrm{Da}$ and polydispersity of 1.33 . Wood pulp was obtained from Xinxiang Natural Chemical Co., Ltd. The viscosityaverage degree of polymerization was estimated to be 870 according to measurements using an Ubbelohde viscometer in cupric ethylene diamine hydroxide solution (CUEN). The lipase from Candida rugosa (CRL) was purchased from Sigma Aldrich, while the proteinase $\mathrm{K}$ from Tritirachium album was supplied by VWR International. The activity of the first was 15-25, while that of the second $40 \mathrm{U} / \mathrm{mg}$. Dichloromethane (DCM) (Molar Chemicals, 99\%), Milli-Q distilled water (Millipore, $\rho>18.2 \mathrm{M} \Omega \mathrm{cm}$ ), sodium phosphate (Alpha Asear, 97\%), sodium chloride (Molar Chemicals, 99.97\%), tris(hydroxymethyl) aminomethane (Molar Chemicals, 99.5\%) (Tris), ortho-phosphoric acid (Molar Chemicals 85.44\%), hydrochloric acid (Molar Chemicals, 36.67\%), lactic acid (VWR International, $1.0 \mathrm{~N}$ aqueous solution) and iron (III) chloride hexahydrate (Riedel-de Haen, min 99\%) were used as received.

\subsubsection{Preparation of the buffer solutions}

Various buffer solutions were prepared with different $\mathrm{pH}$ (7.2 and 8.6) and ionic strength $(\mathrm{I}=12,25$, and $100 \mathrm{mM})$ values. In the case of the phosphate buffer (PBS) $(\mathrm{pH}=7.2), 30 \mathrm{mmol}$ (4.9182 $\mathrm{g}$ ) Na $3 \mathrm{PO}_{4}$ was dissolved in Milli-Q water and $20 \mathrm{mmol}$ $\left(2.3059 \mathrm{~g}, 1.28 \mathrm{~cm}^{3}\right) \mathrm{o}-\mathrm{H}_{3} \mathrm{PO}_{4}$ was added. The exact $\mathrm{pH}$ of the buffer was adjusted with the addition of $\mathrm{HCl}$. The solution was placed into a measuring flask of 11 volume; it was filled up to sign and homogenized.

Buffers of $\mathrm{pH}=8.6$ were prepared with dissolving $50 \mathrm{mmol}(6.057 \mathrm{~g})(\mathrm{I}=12 \mathrm{mM}$, $\mathrm{c}=50 \mathrm{mM})$ or $100 \mathrm{mmol}(12.114 \mathrm{~g})(\mathrm{I}=25 \mathrm{mM}, \mathrm{c}=100 \mathrm{mM})$ Tris in $900 \mathrm{ml} \mathrm{MilliQ}$ water and the $\mathrm{pH}$ of the solution was adjusted with the addition of $\mathrm{HCl}$, finally the solutions were diluted to 11 in a measuring flask. The third tris buffer $(I=100 \mathrm{mM}$, 
$\mathrm{c}=100 \mathrm{mM}$ ) was prepared with same method as the buffer with $\mathrm{I}=25 \mathrm{mM}$ just $7.3 \mathrm{mmol}(0.427 \mathrm{~g}) \mathrm{NaCl}$ was added to increase the ionic strength without changing buffer concentration.

\subsubsection{Preparation of cellulose nanocrystals}

Hydrochloric acid was poured into a desiccator. The valve of the desiccator was left open for a day for $\mathrm{HCl}$ to fill the vessel completely. Wood pulp was placed into the desiccator next and left standing for $8 \mathrm{~h}$. Excessive $\mathrm{HCl}$ was washed from the pulp afterwards, and then the pulp was subjected to ultrasonic treatment and high-pressure homogenization. The product was re-dispersed with sonication for $10 \mathrm{~min}$. The cellulose content of the obtained dispersion was $1.01 \mathrm{wt} . \%$. The particles obtained are typically rigid rod-shaped monocrystalline cellulose domains with about $15 \mathrm{~nm}$ in diameter and $360 \mathrm{~nm}$ in length ${ }^{36}$.

\subsubsection{Preparation of PLA/CNC nanocomposites}

The dispersion of cellulose nanocrystals was diluted with deionized water to achieve concentrations of $0.25,0.5$ and $0.75 \mathrm{w} / \mathrm{v} \%$. A PLA solution of $100 \mathrm{mg} / \mathrm{ml}$ concentration was prepared in dichloromethane. $10 \mathrm{ml}$ of this solution was added to the CNC suspension and then the emulsion obtained was stirred at $12,000 \mathrm{rpm}$ for $3 \mathrm{~min}$ and then sonicated for $3 \mathrm{~min}$. Dichloromethane was evaporated from the system at ambient temperature in $24 \mathrm{~h}$. The remaining material was filtered with a 500-mesh vacuum filter. The obtained filtrate was dried under vacuum at $60{ }^{\circ} \mathrm{C}$ for $24 \mathrm{~h}$. The composite powder was compression molded into plates at $180^{\circ} \mathrm{C}$ and $5 \mathrm{~min}$. The composites contained 5, 10 and $15 \mathrm{wt} . \% \mathrm{CNC}$, respectively.

\subsubsection{Methods and characterization}

The $\mathrm{pH}$ of the buffers was determined using a Metrohm 827 type apparatus with combined glass electrode. The crystalline structure of the PLA/CNC nanocomposites was characterized by X-ray diffraction measurements using a Phillips PW 1830 equipment with $\mathrm{CuK} \alpha$ irradiation at $40 \mathrm{KV}$ and $35 \mathrm{~mA}$ anode excitation between 5 and $40^{\circ} 2 \theta$ angles with $0.04^{\circ}$ step scan rate.

\subsubsection{Degradation studies}

The compression molded plates of neat PLA and the composites were cut to pieces of $15 \times 15 \mathrm{~mm}$ dimensions. The samples were placed into vials and the enzyme solution or the buffer (reference) was measured onto the specimens. The vials were 
shaken at $200 \mathrm{rpm}$ and $37^{\circ} \mathrm{C}$ afterwards. Three parallel measurements were done during the preliminary experiments, while individual samples were investigated in the other cases. In the preliminary experiments, the pieces were taken from the vial at certain time intervals, washed with water, wiped, weighed, and then re-immersed into the same solution. In the other experiments, the samples were taken from the vial after a certain time, washed with water, wiped, dried in a vacuum oven at $25^{\circ} \mathrm{C}$ and $200 \mathrm{mbar}$ for $12 \mathrm{~h}$ and weighed. The concentration of the enzyme was selected according to literature references ${ }^{21,25,31}$.

Besides the measurement of weight loss, degradation was followed also by the determination of the main degradation product, lactic acid. The monomer forms a complex with iron(III) chloride hexahydrate which has an adsorption maximum at $390 \mathrm{~nm}$ in the UV-vis spectrum ${ }^{37} .3 \mathrm{ml}$ reagent solution $(3 \mathrm{mg} / \mathrm{ml})$ was added to $75 \mu \mathrm{l}$ degradation medium to form the complex. Background was recorded with $3 \mathrm{ml}$ reagent and $75 \mu \mathrm{l}$ buffer. Spectra were recorded using a Unicam UV-500 apparatus between 190 and $600 \mathrm{~nm}$ at $600 \mathrm{~nm} / \mathrm{min}$ scan rate and $1 \mathrm{~nm}$ resolution in a quartz cuvette of $1 \mathrm{~cm}$ thickness. 


\section{References}

[1] de Andrade Coutinho, P. L.; Morita, A. T.; Cassinelli, L. F.; Morschbacker, A.; Do Carmo, R. W. Braskem's ethanol to polyethylene process development. Catalytic Process Development for Renewable Materials 2013, 151-167.

[2] Scott, A. Lego plants to be made from plants. Chemical \& Engineering News 2018, 96 (11), 16-16.

[3] Miller, S. A., Sustainable polymers: opportunities for the next decade. ACS Publications: 2013.

[4] Imre, B.; Pukánszky, B. Compatibilization in bio-based and biodegradable polymer blends. European Polymer Journal 2013, 49 (6), 1215-1233.

[5] Ulonska, K.; König, A.; Klatt, M.; Mitsos, A.; Viell, J. r. Optimization of multiproduct biorefinery processes under consideration of biomass supply chain management and market developments. Industrial \& Engineering Chemistry Research 2018, 57 (20), 6980-6991.

[6] Eerhart, A.; Faaij, A.; Patel, M. Replacing fossil based PET with biobased PEF; process analysis, energy and GHG balance. Energy \& Environmental Science 2012, 5 (4), 6407-6422.

[7] Garlotta, D. A literature review of poly (lactic acid). Journal of Polymers and the Environment 2001, 9 (2), 63-84.

[8] Liu, H.; Zhang, J. Research progress in toughening modification of poly (lactic acid). Journal of Polymer Science Part B: Polymer Physics 2011, 49 (15), 10511083.

[9] Na, Y.-H.; He, Y.; Shuai, X.; Kikkawa, Y.; Doi, Y.; Inoue, Y. Compatibilization effect of poly ( $\varepsilon$-caprolactone)-b-poly (ethylene glycol) block copolymers and phase morphology analysis in immiscible poly (lactide)/poly ( $\varepsilon$-caprolactone) blends. Biomacromolecules 2002, 3 (6), 1179-1186.

[10] Bledzki, A. K.; Jaszkiewicz, A.; Scherzer, D. Mechanical properties of PLA composites with man-made cellulose and abaca fibres. Composites Part A: Applied Science and Manufacturing 2009, 40 (4), 404-412.

[11] Pappu, A.; Pickering, K. L.; Thakur, V. K. Manufacturing and characterization of sustainable hybrid composites using sisal and hemp fibres as reinforcement of poly (lactic acid) via injection moulding. Industrial Crops and Products 2019, 137, 260-269. 
[12] Zhou, L.; He, H.; Li, M.-c.; Huang, S.; Mei, C.; Wu, Q. Enhancing mechanical properties of poly (lactic acid) through its in-situ crosslinking with maleic anhydride-modified cellulose nanocrystals from cottonseed hulls. Industrial Crops and Products 2018, 112, 449-459.

[13] Müller, P.; Imre, B.; Bere, J.; Móczó, J.; Pukánszky, B. Physical ageing and molecular mobility in PLA blends and composites. Journal of Thermal Analysis and Calorimetry 2015, 122 (3), 1423-1433.

[14] Ling, Z.; Zhang, X.; Yang, G.; Takabe, K.; Xu, F. Nanocrystals of cellulose allomorphs have different adsorption of cellulase and subsequent degradation. Industrial Crops and Products 2018, 112, 541-549.

[15] Szabo, O. E.; Csiszar, E. Some factors affecting efficiency of the ultrasoundaided enzymatic hydrolysis of cotton cellulose. Carbohydrate Polymers 2017, 156, 357-363.

[16] Ghorpade, V. M.; Gennadios, A.; Hanna, M. A. Laboratory composting of extruded poly (lactic acid) sheets. Bioresource Technology 2001, 76 (1), 57-61.

[17] Luzi, F.; Fortunati, E.; Puglia, D.; Petrucci, R.; Kenny, J.; Torre, L. Study of disintegrability in compost and enzymatic degradation of PLA and PLA nanocomposites reinforced with cellulose nanocrystals extracted from Posidonia Oceanica. Polymer Degradation and Stability 2015, 121, 105-115.

[18] Masaki, K.; Kamini, N. R.; Ikeda, H.; Iefuji, H. Cutinase-like enzyme from the yeast Cryptococcus sp. strain S-2 hydrolyzes polylactic acid and other biodegradable plastics. Applied and Environmental Microbiology 2005, 71 (11), 7548 .

[19] Pranamuda, H.; Tsuchii, A.; Tokiwa, Y. Poly (L-lactide)-degrading enzyme produced by amycolatopsis sp. Macromolecular Bioscience 2001, 1 (1), 25-29.

[20] Tokiwa, Y.; Calabia, B. P. Biodegradability and biodegradation of poly (lactide). Applied Microbiology and Biotechnology 2006, 72 (2), 244-251.

[21] Żenkiewicz, M.; Richert, A.; Malinowski, R.; Moraczewski, K. A comparative analysis of mass losses of some aliphatic polyesters upon enzymatic degradation. Polymer Testing 2013, 32 (2), 209-214.

[22] Rytlewski, P.; Stepczyńska, M.; Gohs, U.; Malinowski, R.; Budner, B.; Żenkiewicz, M. Flax fibres reinforced polylactide modified by ionizing radiation. Industrial Crops and Products 2018, 112, 716-723. 
[23] Doukyu, N.; Ogino, H. Organic solvent-tolerant enzymes. Biochemical Engineering Journal 2010, 48 (3), 270-282.

[24] Ogino, H.; Miyamoto, K.; Ishikawa, H. Organic-solvent-tolerant bacterium which secretes organic-solvent-stable lipolytic enzyme. Applied and Environmental Microbiology 1994, 60 (10), 3884.

[25] Stepczyńska, M.; Rytlewski, P. Enzymatic degradation of flax-fibers reinforced polylactide. International Biodeterioration \& Biodegradation 2018, 126, 160166.

[26] Williams, D. F. Enzymic hydrolysis of polylactic acid. Engineering in Medicine 1981, $10(1), 5-7$.

[27] Gan, Z.; Yu, D.; Zhong, Z.; Liang, Q.; Jing, X. Enzymatic degradation of poly ( $\varepsilon$-caprolactone)/poly (DL-lactide) blends in phosphate buffer solution. Polymer 1999, 40 (10), 2859-2862.

[28] Lee, S. H.; Kim, I. Y.; Song, W. S. Biodegradation of polylactic acid (PLA) fibers using different enzymes. Macromolecular Research 2014, 22 (6), 657-663.

[29] Malwela, T.; Ray, S. S. Enzymatic degradation behavior of nanoclay reinforced biodegradable PLA/PBSA blend composites. International Journal of Biological Macromolecules 2015, 77, 131-142.

[30] Nakajima-Kambe, T.; Edwinoliver, N.; Maeda, H.; Thirunavukarasu, K.; Gowthaman, M.; Masaki, K.; Mahalingam, S.; Kamini, N. Purification, cloning and expression of an Aspergillus niger lipase for degradation of poly (lactic acid) and poly (E-caprolactone). Polymer Degradation and Stability 2012, 97 (2), 139144.

[31] Dogan, S.; Boyacioglu, S.; Kodal, M.; Gokce, O.; Ozkoc, G. Thermally induced shape memory behavior, enzymatic degradation and biocompatibility of PLA/TPU blends:"Effects of compatibilization". Journal of the Mechanical Behavior of Biomedical Materials 2017, 71, 349-361.

[32] Tsuji, H.; Ikarashi, K. In vitro hydrolysis of poly (l-lactide) crystalline residues as extended-chain crystallites: III. Effects of $\mathrm{pH}$ and enzyme. Polymer Degradation and Stability 2004, 85 (1), 647-656.

[33] Persat, A.; Chambers, R. D.; Santiago, J. G. Basic principles of electrolyte chemistry for microfluidic electrokinetics. Part I: acid-base equilibria and $\mathrm{pH}$ buffers. Lab on a Chip 2009, 9 (17), 2437-2453. 
[34] Michaelis, L.; Menten, M. L. Die kinetik der invertinwirkung. Biochem. $z$ 1913, 49 (333-369), 352.

[35] Polyák, P.; Dohovits, E.; Nagy, G. N.; Vértessy, B. G.; Vörös, G.; Pukánszky, B. Enzymatic degradation of poly-[(R)-3-hydroxybutyrate]: Mechanism, kinetics, consequences. International Journal of Biological Macromolecules 2018, 112, 156-162.

[36] Zhang, Y.; Cui, L.; Xu, H.; Feng, X.; Wang, B.; Pukánszky, B.; Mao, Z.; Sui, X. Poly (lactic acid)/cellulose nanocrystal composites via the Pickering emulsion approach: Rheological, thermal and mechanical properties. International Journal of Biological Macromolecules 2019, 137, 197-204.

[37] Borshchevskaya, L.; Gordeeva, T.; Kalinina, A.; Sineokii, S. Spectrophotometric determination of lactic acid. Journal of Analytical Chemistry 2016, 71 (8), 755758. 


\section{Chapter}

Foaming of Biodegradable Polylactic Acid/Cellulose Nanocrystal Composites: Pickering Emulsion Templating to Enhance Filler Dispersion and Engineer Foam Morphology

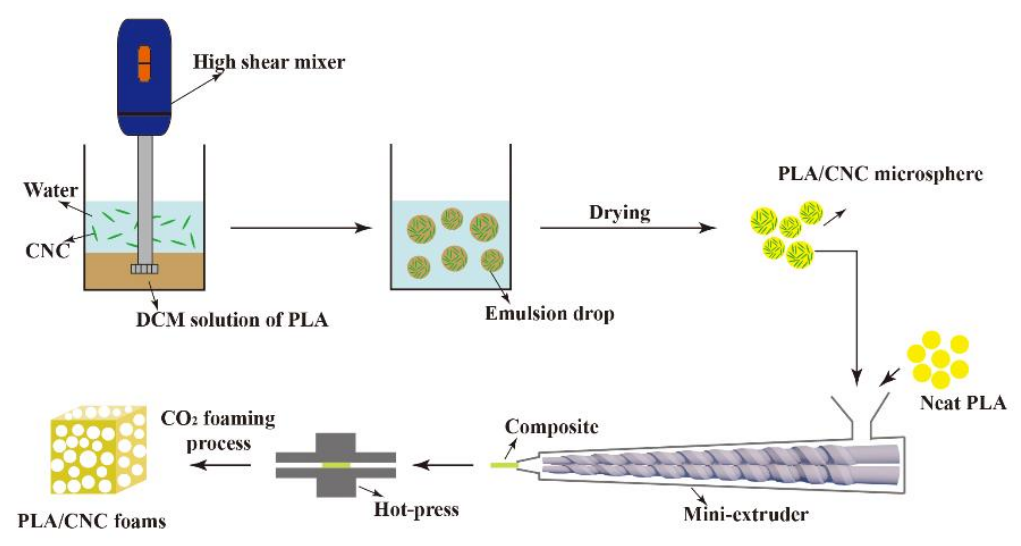


Polylactic acid (PLA)/cellulose nanocrystal (CNC) composite foams have attracted much attention due to their biodegradability and potential to replace petroleum-based foams. However, the dispersion of (unmodified) CNCs in PLA remains challenging. Here we used the Pickering emulsion templating method of unmodified CNCs to stabilize PLA microspheres and to prepare a concentrated masterbatch. This masterbatch was used in conventional melt blending of pristine PLA to obtain well-dispersed CNCs in PLA with a CNC loading of up to $5 \mathrm{wt} \%$. In comparison, PLA/CNC composites prepared by direct melt blending of CNCs in PLA showed visual agglomerates of CNCs. The PLA/CNC composites were batch foamed with $\mathrm{CO}_{2}$ as a physical blowing agent. We demonstrate that the good $\mathrm{CNC}$ dispersion obtained by the Pickering emulsion route is favorable for the preparation of PLA foams with smaller and more uniform cell sizes and with a higher cell density. These results demonstrate that the Pickering emulsion approach to prepare $\mathrm{CNC}$ masterbatches for subsequent dispersion in thermoplastics by conventional processing seems promising for numerous industrial applications.

\footnotetext{
*The contents of this chapter have been submitted (under review) in: Yunchong Zhang, Joost Duvigneau, Xiaofeng Sui and G. Julius Vancso; Foaming of Biodegradable Polylactic Acid/Cellulose Nanocrystal Composites: Pickering Emulsion Templating to Enhance Filler Dispersion and Engineer Foam Morphology.
} 


\subsection{Introduction}

Polymer foams are widely used in many fields, such as food packaging, building construction, transportation, electronics, and so on, due to their lightweight, thermal and sound insulating, and impact energy dissipating properties ${ }^{1-3}$. At present, most polymer foam products consist of petroleum-based materials, such as polystyrene (PS), polyurethane (PU), polyvinyl chloride (PVC), and poly (methyl methacrylate) (PMMA) foams. To achieve biodegradability and to recycle these systems remain a grand challenge. In addition, the unavoidable fossil resource depletion and increasing environmental concerns direct researchers to develop biodegradable polymer foams to replace currently used petroleum-based materials ${ }^{4-5}$.

Polylactic acid (PLA), an aliphatic thermoplastic polyester, is produced from renewable resources, such as cornstarch and sugarcane ${ }^{6-7}$. PLA has attracted distinguished industrial and academic interest over recent years, attributed to its biodegradability, biocompatibility, and good mechanical properties. Therefore, PLA is considered as a promising substitute for some petroleum-based foamed products ${ }^{8}$. However, PLA has several disadvantages, i.e., low melt strength, poor thermal resistance, and brittleness, which significantly limit the commercial exploitation of PLA-based foams ${ }^{9-10}$. Some strategies to improve PLAs' foaming behavior and foam properties include blending with other polymers ${ }^{11-12}$, introducing a long-chain branched structure ${ }^{13}$, and adding particles ${ }^{14}$. Among these methods, the addition of particles is a well-known, practical, and straightforward way to improve PLAs' foaming behavior and foam properties ${ }^{10,15-16}$.

Nanocellulose, derived from renewable resources, is well known for its high strength, biocompatibility, and biodegradability ${ }^{17-19}$. Therefore, nanocellulose is considered as a promising particle to improve the foaming behavior and foam properties of PLA when used as Pickering emulsifier. During PLA foaming, welldispersed nanocellulose particles can enhance the melt strength of PLA by forming a nanocellulose network in the PLA matrix by hydroxyl bonds and entanglements between them ${ }^{20}$. Furthermore, nanocellulose could act as heterogeneous nucleation sites to increase the cell density of PLA foams ${ }^{21}$. For instance, Park and coworkers ${ }^{17}$ reported the preparation of PLA/cellulose nanofibers (CNF) composite foams. They studied the effect of added CNFs on the rheological and thermal properties of the foams and the composite foaming behavior. They observed that the addition of CNFs increased the viscosity of PLA in the low-frequency range of rheology measurements, led to higher cell densities, decreased cell sizes of the composite foams, and enhanced 
the crystallization kinetics of PLA.

The degree of nanocellulose dispersion in the PLA matrix significantly influences the PLA foam morphology and properties. During the foaming process, the foam cell nucleating efficiency of nanocellulose is decreased when particles are poorly dispersed in the PLA matrix ${ }^{14}$. However, due to the poor compatibility of cellulose and PLA, it is difficult to disperse nanocellulose uniformly ${ }^{22}$. Chemical surface modification of nanocellulose is a well-known and frequently applied strategy to improve its dispersion in PLA ${ }^{23}$. For instance, Wu and coworkers ${ }^{24}$ compared the cell morphology and mechanical properties of PLA/cellulose nanocrystal (CNC) composite foams with three different types of $\mathrm{CNCs}$, including pristine $\mathrm{CNC}$, and acetylated ones with lower and moderate degrees of substitution. The composites with acetylated-CNCs had a better CNC dispersion than those with pristine CNCs. The mechanical properties and foaming behavior of foams were improved due to the enhanced CNC dispersion and increased affinity between CNC and PLA. Alternatively to chemical surface modification strategies, our group has developed a Pickering emulsion-based method to prepare nanocellulose polymer composites with a good particle dispersion ${ }^{25-28}$. However, the Pickering emulsion method requires a typical particle loading exceeding $5 \mathrm{wt} . \%$, which is relatively high for PLA/CNC nanocomposite foams. In addition, when processing the CNC covered PLA micropowder obtained by the Pickering emulsion method, CNC-rich and CNC-poor domains form with preference, when no additional mixing is applied. Therefore, in this Chapter, we report the extension of our earlier work to disperse pristine CNCs in PLA by first preparing a Pickering emulsion-based PLA/CNC masterbatch with a CNC loading of $10 \mathrm{wt} . \%$. In a second step, the CNC concentration is diluted to below 5 wt.\% by conventional melt mixing of the masterbatch with PLA in an extruder. Melt mixing introduces additional dispersive forces that distribute the CNCs homogeneously throughout the PLA matrix. The composites obtained were foamed by $\mathrm{CO}_{2}$ batch foaming. We will demonstrate that this straightforward method is very convenient to prepare PLA/CNC composites with a uniform particle dispersion without using the typically required particle surface modification strategies. In addition, we will show that a good CNC distribution encompassing unmodified cellulose is beneficial in PLA foam cell nucleation and foam cell stabilization.

\subsection{Results and Discussion}

PLA/CNC composite films with different CNC concentrations were prepared by 
the Pickering emulsion masterbatch route as is shown in Figure 6.1a. In a first step, PLA/CNC composite microspheres with a high CNC concentration (10 wt.\%), to serve as a masterbatch, were made by Pickering emulsion-based templating. Secondly, through melt blending by a mini-extruder, PLA/CNC composites with dispersed CNCs at particle loadings below 5 wt.\% were prepared by blending the PLA/CNC masterbatch with neat PLA. This route is referred to as the Pickering emulsion masterbatch route. Finally, composite films were made by hot-pressing. In the next step, neat PLA and PLA/CNC composites were foamed using a two-step $\mathrm{CO}_{2}$ batch foaming process (Figure 6.1b).

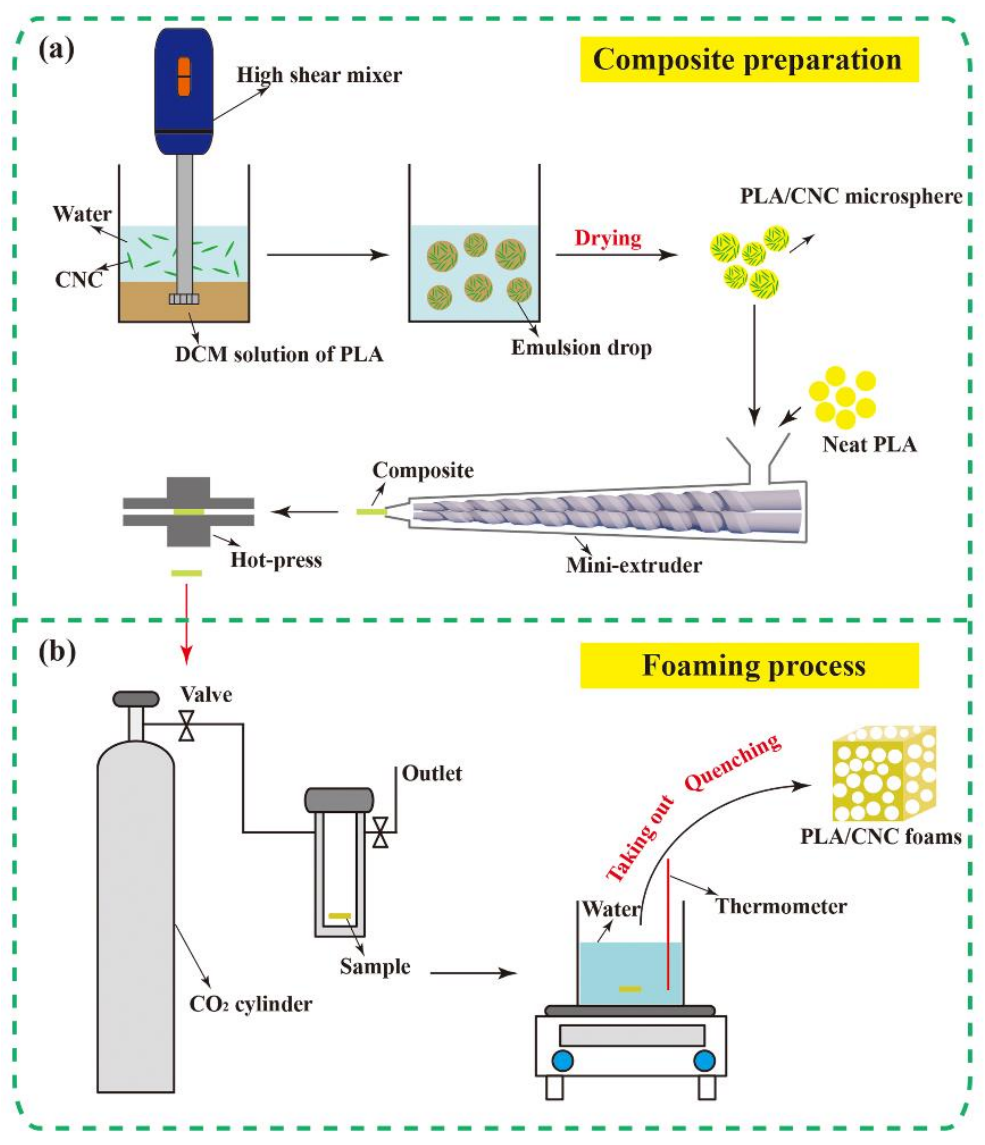

Figure 6.1 Schematic of (a) the PLA/CNC composite film preparation method and (b) the twostep $\mathrm{CO}_{2}$ batch foaming process.

\subsubsection{CNC dispersion in the PLA matrix}

The quality of the CNC dispersion in the PLA matrix has, as we will show later, a 
significant influence on the PLA foamability and foam properties. Figure 6.2 shows a photograph of neat PLA as well as of PLA/CNC composite films prepared by i) the Pickering emulsion masterbatch route, ii) direct melt blending, and iii) Pickering emulsion-based templating for various $\mathrm{CNC}$ loadings. No visible $\mathrm{CNC}$ aggregation is observed in the PLA/CNC composite films prepared by the Pickering emulsion masterbatch route. Before melt blending, CNCs were evenly distributed at the surface of the PLA microspheres (masterbatch) ${ }^{29}$. Furthermore, the PLA/CNC composite films prepared using only Pickering emulsion templating show a good CNC dispersion, which is consistent with our previous reported results ${ }^{25}$.

However, many CNC aggregates are observed in the PLA/CNC composite films when using direct melt blending to prepare the composites. Some CNC agglomerates formed during the freeze-drying process, and simple melt blending was insufficient to break these agglomerates. This observation agrees with results reported by Bendahou and coworkers ${ }^{30}$ for the dispersion of unmodified CNC powder in PLA by extrusion blending.

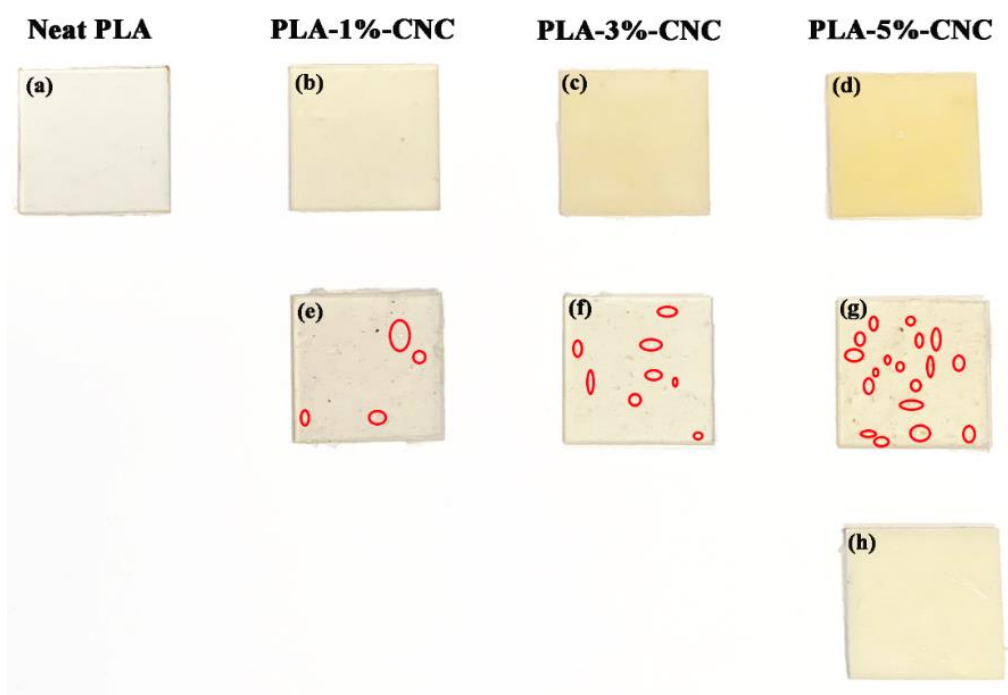

Figure 6.2 Photograph of PLA/CNC composite films with different CNC content (up to 5 wt.\%) prepared by the Pickering emulsion masterbatch route (a-d), direct melt blending (e-g), and Pickering emulsion-based templating (h). Some visually observable CNC agglomerates are enclosed in the red circles.

In addition, it is observed that the PLA films became slightly yellowish after the addition of CNCs. This is attributed to the low thermal stability of CNCs with 
sulfonate groups, which undergo thermal degradation during the extrusion process ${ }^{31}$. Although the processing temperature is not as high as the onset temperature of $\mathrm{CNC}$ degradation $\left(\sim 220^{\circ} \mathrm{C}\right)$, the thermal shear experienced by the $\mathrm{CNCs}$ during twin-screw extrusion is expected to induce $\mathrm{CNC}$ degradation. This may explain why the composite films prepared by the Pickering emulsion masterbatch route are more colored compared to the films prepared by Pickering emulsion-based templating and subsequent compression molding.

To further investigate the $\mathrm{CNC}$ dispersion in the PLA matrix, the cross-sections of PLA/CNC composites with 5 wt.\% CNC contents prepared by the different methods were observed by SEM. SEM images are shown in Figure 6.3. In the SEM images of the composites prepared by the Pickering emulsion masterbatch route (Figure 6.3ab), there are no visible $\mathrm{CNC}$ agglomerates present, indicating that the $\mathrm{CNCs}$ were well-dispersed in the composite. In SEM imaging there is no contrast between the dispersed CNCs and the PLA matrix. We were unable to resolve the few tens of nanometer thick and several hundred $\mathrm{nm}$ long $\mathrm{CNCs}$ even in high resolution SEM imaging, as is shown in Figure 6.1a (Figure 6-S1 shows AFM images of the used CNCs deposited on silicon substrates). However, in the SEM images of the composites prepared by the direct melt blending method (Figure 6.3c-d), CNC agglomerates are readily observed. This indicates that the CNC agglomerates in the freeze-dried powder are not easily broken up and become dispersed by just melt blending. 

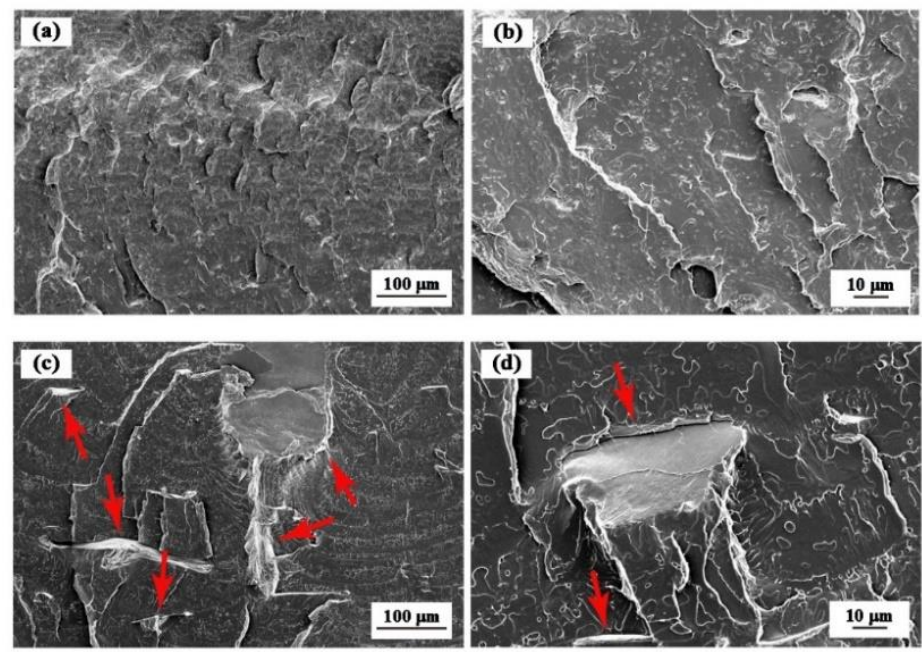

Figure 6.3 SEM images of cross-sectioned PLA/CNC composites with a $5 \mathrm{wt} . \% \mathrm{CNC}$ loading prepared by $(a-b)$ the Pickering emulsion masterbatch route and (c-d) the direct melt blending method. The red arrows point at agglomerated $\mathrm{CNC}$ particles.

\subsubsection{PLA/CNC composite foaming: The influence of CNC dispersion}

PLA/CNC composites prepared by the different methods were foamed by a twosteps $\mathrm{CO}_{2}$-based batch foaming process. The $\mathrm{CO}_{2}$ uptake of PLA/CNC composites with a $\mathrm{CNC}$ content between 0 to $5 \mathrm{wt} . \%$ after $6 \mathrm{~h} \mathrm{CO}_{2}$ saturation at 40 bars is shown in Figure 6-S2. The $\mathrm{CO}_{2}$ concentration after saturation was around $20 \mathrm{wt} \% \%$ for all samples. The modest decrease in $\mathrm{CO}_{2}$ uptake ( 1.5 wt.\%) upon increasing the $\mathrm{CNC}$ concentration is attributed to the highly crystalline nature of the CNCs, which prevents $\mathrm{CO}_{2}$ absorption by the CNCs in the PLA matrix.

Upon saturation with $\mathrm{CO}_{2}$, the PLA becomes plasticized, which for some PLA grades may induce crystallization ${ }^{15}$. It is known that PLA crystals can act as heterogeneous foam cell nucleating agents ${ }^{32}$ and/or may limit the subsequent cell growth during batch foaming. Therefore, we performed DSC measurements of PLA/CNC samples after saturation with $\mathrm{CO}_{2}$ for 6 hours to determine whether the PLA became partially crystallized before foam cell nucleation. The corresponding DSC results are shown in Figure 6-S3. In the first DSC heating traces there are no melting peaks observed for the PLA/CNC composites, indicating that no PLA crystals formed during $6 \mathrm{~h} \mathrm{CO}_{2}$ saturation. Noteworthy is that the glass transition temperatures of the PLA/CNC composites are higher than that of neat PLA. This is because the 
dispersed CNCs decrease the mobility of PLA chains ${ }^{25,33-34}$. The lower mobility of PLA chains would help to reduce foam cell expansion, as well.

Following saturation with $\mathrm{CO}_{2}$, foam cells in the PLA/CNC composites were nucleated and the material became foamed. The following section will present and discuss the effect of the quality of the CNC dispersion in the PLA matrix on the foaming behavior and foam morphology.

Figure 6.4 shows SEM images of cross-sectioned neat PLA and PLA/CNC composite foams. The corresponding cell size distributions, mean cell sizes, and cell densities are shown in Figure 6.5. The SEM images of the cross-sectioned foams qualitatively show that neat PLA foams (Figure 6.4a) have a poor quality foam morphology with many coalesced and collapsed cells. ${ }^{35}$ Upon adding up to $5 \mathrm{wt} . \%$ CNCs by the Pickering emulsion masterbatch route, the PLA/CNC composite foams have a decreased cell size with an increased cell density. As is shown in Figure 6S4a-d, it is observed that cell size distribution becomes narrower as the CNC content increases. The PLA/CNC foams obtained from the composites prepared by the masterbatch route with a CNC content of $5 \mathrm{wt} . \%$ had the lowest mean cell size (36 $\mu \mathrm{m}$, see Figure 6.5a) and the narrowest cell size distribution, i.e. with cell sizes between 15 to $86 \mu \mathrm{m}$. In addition, the cell density of the PLA/CNC composite foams increased from $2.6 \times 10^{6}$ cells $/ \mathrm{cm}^{3}$ to $7.4 \times 10^{6}$ cells $/ \mathrm{cm}^{3}$ upon increasing the $\mathrm{CNC}$ content from 1 w. t $\%$ to 5 wt.\%, respectively (See Figure 6.5b). We attribute the decrease in cell size, the narrower cell size distribution, and the increased cell density values for PLA/CNC foams prepared by the masterbatch route to well-dispersed CNCs in the PLA matrix. This is related to a more effective nucleation of a large number of foam cells ${ }^{24,36}$ and to enhanced melt viscosity of the PLA to moderate foam cell expansion.

In contrast, it is clear from Figure 6.4e-g and Figure 6.5 that the PLA/CNC composites prepared by direct melt blending show a modest decrease in cell size and that the cell density remained of the same order of magnitude as the cell density of the pristine PLA foams. Furthermore, the cell size distribution remained relatively broad. We attribute these observations to the poor CNC dispersion. As such, the number of potential nucleating sites is reduced, and the lower number of dispersed CNCs are less effective in reducing cell growth.

We also foamed PLA/CNC composites with 5 wt.\% CNC prepared by compression molding of CNC-covered PLA particles obtained by the Pickering emulsion templating route as a control experiment. To our surprise, these PLA/CNC 
composite films did not foam well (Figue 6.4h). A possible explanation for this poor foaming behavior is provided below. First, we note that CNCs remain at the surface of dried PLA spheres (diameter $20 \mu \mathrm{m}$ ) prepared by the Pickering emulsion method. Next, we assume that by compression molding in the absence of dispersive forces, the CNCs form well-dispersed CNC-rich domains at the fused particle interphases enclosing CNC-poor domains (i.e. the former PLA sphere interior). This means that compression molding of the CNC-covered PLA spheres induces the formation of a network-like structure of CNC-rich domains throughout the composite. In addition, the CNC-rich domains probably have a sufficiently high melt viscosity that foam cell expansion in the enclosed PLA-rich domains is hindered.

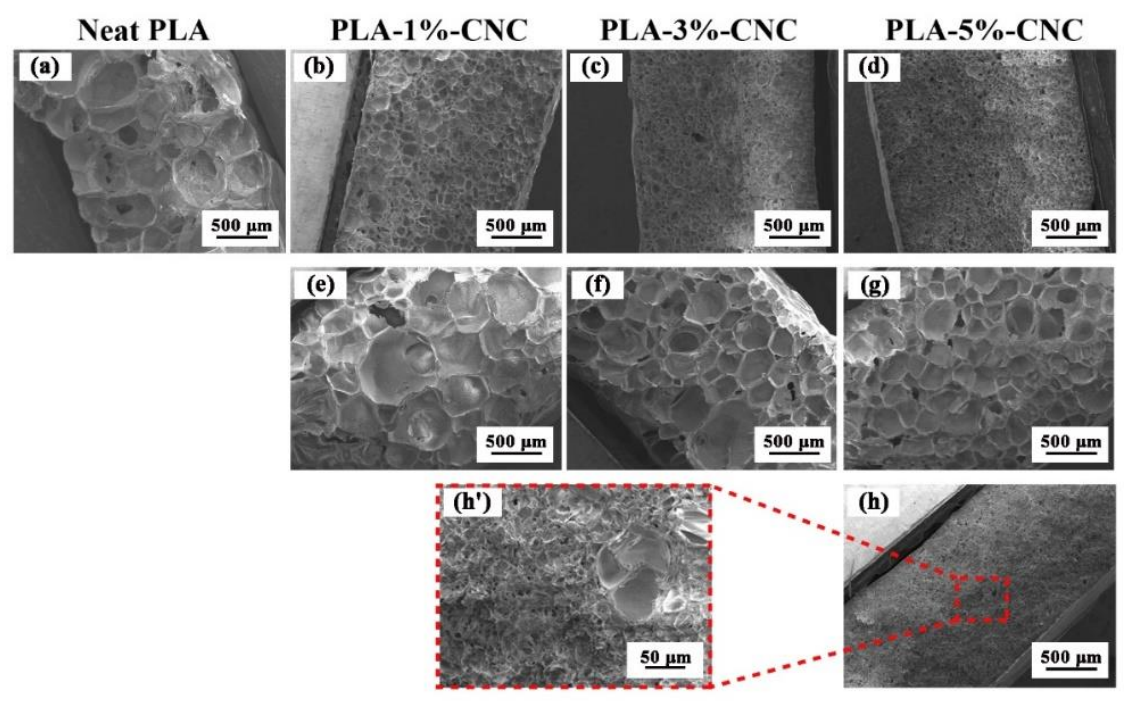

Figure 6.4 SEM images of cross-sectioned PLA/CNC composite foams with different CNC content (up to $5 \mathrm{wt}$ \%) prepared by the Pickering emulsion masterbatch route (a-d), direct melt blending (e-g), and Pickering emulsion-based templating (h). 

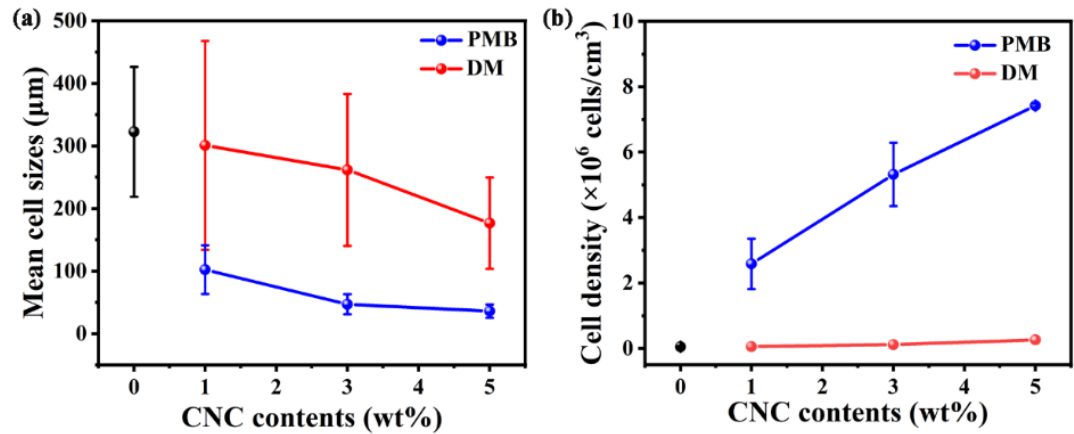

Figure 6.5 Mean cell size (a) and cell density (b) of PLA/CNC composite foams of composite films prepared by the masterbatch route (PMB) and direct melt blending (DM) as a function of the $\mathrm{CNC}$ content.

Based on the quantitative and qualitative foam morphology analysis presented and discussed above, we conclude that the Pickering emulsion masterbatch route provides a promising avenue for the dispersion of unmodified CNCs in a more hydrophobic PLA matrix. This opens possibilities to explore the effect of adding unmodified welldispersed CNCs to PLA to further improve the foam morphology. The following two sections focus on PLA/CNC foams obtained by different foaming temperatures and foaming times.

\subsubsection{The influence of foaming temperature on PLA/CNC foaming}

Besides $\mathrm{CNC}$ dispersion and loading, the foaming temperature also has a significant influence on PLA foaming. Figure 6.6 shows SEM images of PLA/CNC films with a CNC loading of up to $5 \mathrm{wt} \% \%$ foamed at 40,50 , and $60{ }^{\circ} \mathrm{C}$, respectively. As is shown in Figure 6.6, for a foaming temperature above $40^{\circ} \mathrm{C}$, the cells of pristine PLA foams were ruptured due to fast cell growth, or collapsed due to the lack of PLA melt strength. Remarkably, upon the addition of 1 to $5 \mathrm{wt} . \%$ well-dispersed CNCs, the melt strength of the PLA in combination with the nucleating effect of CNCs resulted in the formation of PLA/CNC foams with a closed-cell morphology.

Figure 6.7a shows the average cell size of the foams obtained at different temperatures. Upon increasing the foam temperature from $40{ }^{\circ} \mathrm{C}$ to $60{ }^{\circ} \mathrm{C}$, the mean cell size of PLA/CNC foams with 5 wt.\% CNC increased from $36 \mu \mathrm{m}$ to $74 \mu \mathrm{m}$, respectively. The cell size distribution of PLA/CNC composite foams with different CNC content at different foaming temperatures is provided in Figure 6-S5. The cell density (Figure 6.7b) of the PLA/CNC foams with 5 wt.\% CNC decreased by $66.7 \%$ 
(from $7.4 \times 10^{6}$ cells $/ \mathrm{cm}^{3}$ to $2.5 \times 10^{6}$ cells $/ \mathrm{cm}^{3}$ ) as the foaming temperature increased from $40{ }^{\circ} \mathrm{C}$ to $60{ }^{\circ} \mathrm{C}$. These observations were expected due to increased cell expansion at higher foaming temperatures resulting in more cell coalescence and foam cell collapse. In addition, it is clear from Figure 6.6 and Figure 6.7 that the addition of unmodified CNCs to PLA matrixes by the Pickering emulsion templated masterbatch route significantly broadens the processing window for PLA foaming.

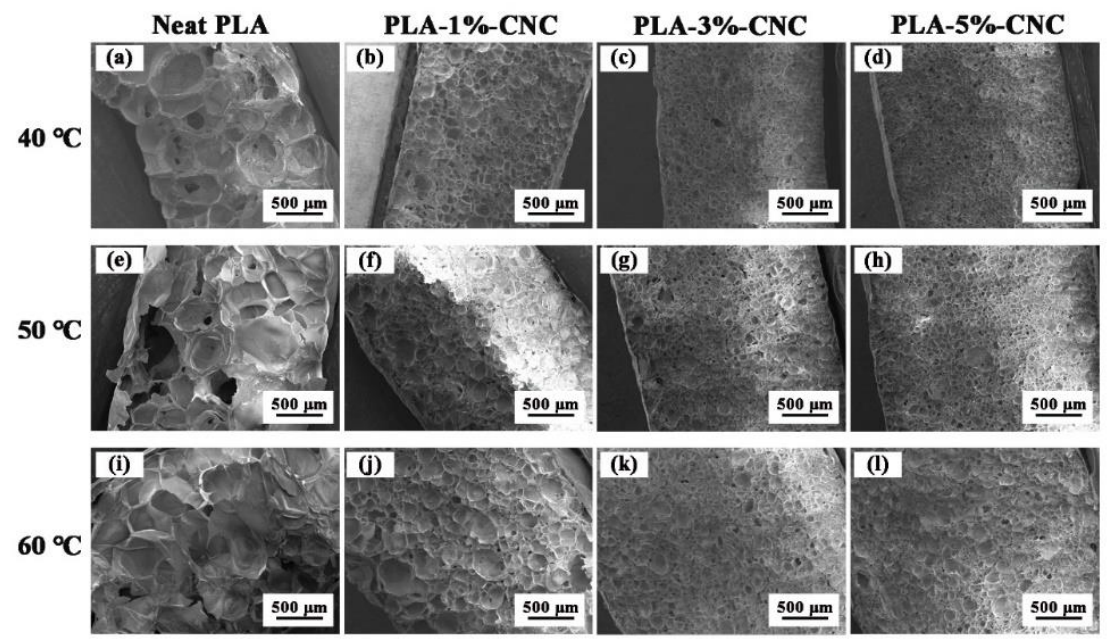

Figure 6.6 SEM images of cross-sectioned PLA/CNC composite foams as a function of the $\mathrm{CNC}$ loading and foaming temperature. The $\mathrm{CNC}$ loading was between 0 to $5 \mathrm{wt} . \%$, and the foaming temperature was 40,50 , or $60^{\circ} \mathrm{C}$.
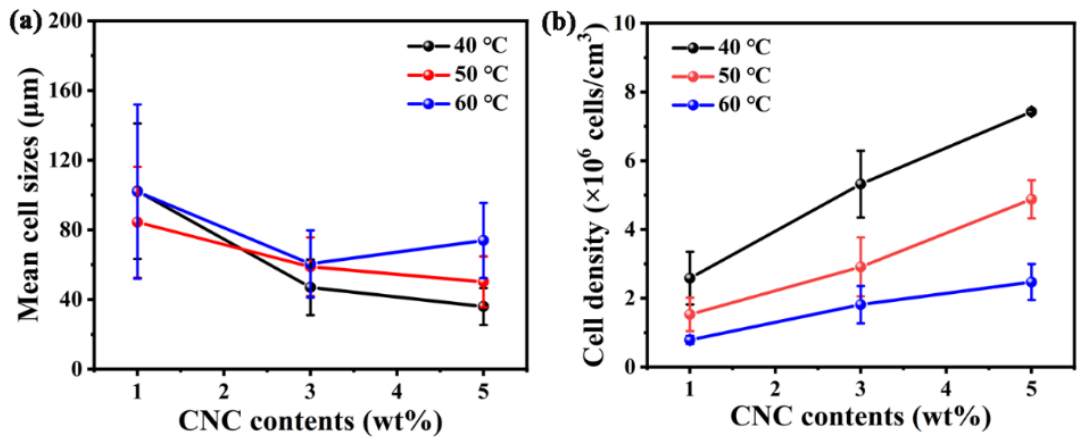

Figure 6.7 Mean cell size (a) and cell density (b) of PLA/CNC composite foams prepared at different foaming temperatures. 


\subsubsection{The influence of foaming time on the PLA/CNC foam morphology}

We investigated the influence of foaming time on the PLA/CNC foam morphology development to establish a processing window for the batch foaming of our PLA/CNC composites. Figure 6.8 shows SEM images of cross-sectioned PLA/CNC foams obtained during $5 \mathrm{~s}, 10 \mathrm{~s}$, or $20 \mathrm{~s}$, respectively. Figure 6.9 shows the corresponding mean cell size and cell density values of the PLA/CNC foams (the cell size distributions are provided in Figure 6-S6). From the quantitative analysis presented in Figure 6.9, it is clear that the foam morphologies are comparable for materials obtained at foaming times of 5 and 10 seconds. Upon increasing the foaming time, the cell size increases significantly while the cell density decreases. For instance, the cell size increased from $18 \mu \mathrm{m}$ to $36 \mu \mathrm{m}$, and the cell density decreased from $11.7 \times 10^{6}$ cells $/ \mathrm{cm}^{3}$ to $7.4 \times 10^{6}$ cells $/ \mathrm{cm}^{3}$, upon increasing the foaming time from 5 to 20 seconds for PLA/CNC composites with 5wt.\% CNCs, respectively. These observations are explained by an increasing foam cell coalescence at longer foaming times.

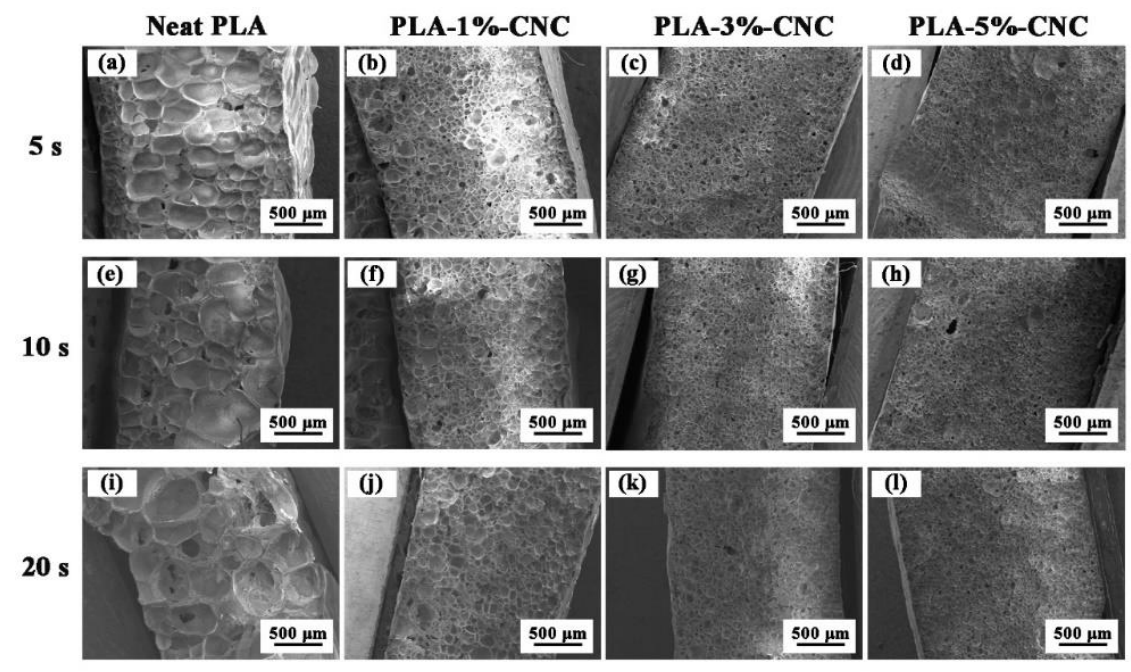

Figure 6.8 SEM images of cross-sectioned PLA/CNC composite foams as a function of the $\mathrm{CNC}$ loading and foaming time. The $\mathrm{CNC}$ loading values were between 0 to $5 \mathrm{wt} . \%$, and the foaming times ranged from 5,10 and 20 seconds. 

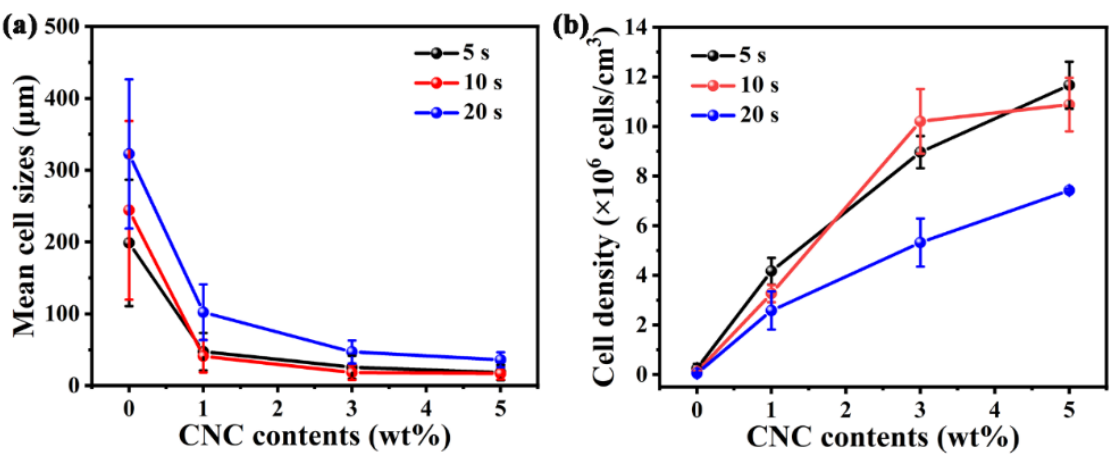

Figure 6.9 Mean cell size (a) and cell density (b) of PLA/CNC composite foams prepared with different foaming times.

Overall we conclude that the cell morphology of PLA/CNC foams is readily adjusted by changing the foaming temperature and time, between $40-60{ }^{\circ} \mathrm{C}$ and $5-10$ seconds, respectively, for the given saturation pressure (40 bar) and saturation time (6 hours). Foaming at $40{ }^{\circ} \mathrm{C}$ for $5 \mathrm{~s}$ resulted in PLA/CNC foams with the lowest mean cell size $(\sim 18 \mu \mathrm{m})$ and highest cell density $\left(\sim 11.7 \times 10^{6}\right)$. Compared to the previous studies of PLA/cellulose composite foams ${ }^{17-19,24,37-41}$, the materials in this work are obtained at low saturation pressure and low foaming temperature, and have a low mean cell size and good cell density.

\subsection{Conclusions}

We prepared PLA/CNC composites with up to $5 \mathrm{wt} . \%$ well-dispersed unmodified CNCs by the Pickering emulsion masterbatch route. In addition, we demonstrated that these unmodified CNCs were successful for foam cell nucleation and foam cell stabilization. The foam morphology was readily tuned by the $\mathrm{CNC}$ content, foaming temperature, and foaming time. When the CNCs were not uniformly dispersed in the PLA matrix, as observed by direct melt blending and Pickering emulsion templating, the resulting foam morphologies were not well-developed within the evaluated processing window. This is either attributed to having a lower number of effective CNC particles as nucleating agents, present in the PLA matrix, or to the formation of CNC-rich and CNC-poor domains by the blending methods. Overall we show that the Pickering emulsion templating masterbatch route allows incorporating a hydrophilic filler in a hydrophobic thermoplastic matrix, which can be easily generalized to other filler/thermoplastic matrix systems. 


\subsection{Experimental sections}

\subsubsection{Materials}

Polylactic acid (PLA) pellets (4060D), an amorphous polymer with an average Dlactide content of $12 \mathrm{wt} . \%$ and a glass transition temperature $\left(\mathrm{T}_{\mathrm{g}}\right)$ of $55-60{ }^{\circ} \mathrm{C}$, were supplied by Natureworks (USA). Cellulose nanocrystals (CNC) were purchased from CelluForce (Canada). Sodium chloride $(\mathrm{NaCl})$ and potassium hydroxide $(\mathrm{KOH})$ were obtained from Sigma-Aldrich. Dichloromethane (DCM, $\left.\mathrm{CH}_{2} \mathrm{Cl}_{2}\right)$ and methanol were obtained from VWR chemicals. All chemicals were used as received.

\subsubsection{Preparation of PLA/CNC composite film}

\section{Pickering emulsion masterbatch route}

A 6 wt.\% CNC dispersion was diluted with deionized water to form a 0.5 wt.\% cellulose dispersion. The CNC to PLA ratio in the Pickering emulsion was set to 10 wt.\% to prepare the PLA/CNC masterbatch. A solution of PLA in DCM (30 ml, 90 $\mathrm{mg} / \mathrm{mL}$ ) was added to the aqueous $\mathrm{CNC}$ dispersion $(60 \mathrm{~mL}) . \mathrm{NaCl}(50 \mathrm{mM})$ was added to the aqueous phase to screen the surface charge of the CNC. The mixture was homogenized (IKA T18 homogenizer, Germany) at 12,000 rpm for $3 \mathrm{~min}$ to obtain Pickering emulsions. DCM was evaporated at ambient temperature for $24 \mathrm{~h}$. The precipitates were collected by vacuum filtration using a 500-mesh filter screen. The obtained PLA/CNC microspheres were vacuum dried at $60{ }^{\circ} \mathrm{C}$ for $24 \mathrm{~h}$. The dried microspheres were mixed with neat PLA pellets by a mini-extruder (DSM) to dilute the $\mathrm{CNC}$ concentration and homogenize the $\mathrm{CNC}$ distribution. The barrel temperature was set to $190{ }^{\circ} \mathrm{C}$, and the screw speed was $100 \mathrm{rpm}$. By this method, PLA/CNC composites with 1, 3 \& 5 wt.\% CNC were prepared. Subsequently, PLA/CNC composite films $\left(20 \mathrm{~mm} * 20 \mathrm{~mm} * 1 \mathrm{~mm}, \mathrm{~L}^{*} \mathrm{~W} * \mathrm{H}\right)$ were prepared by hot-pressing employing a hot-press (Fontijne, the Netherlands) at $180{ }^{\circ} \mathrm{C}$ and $100 \mathrm{KN}$ for $5 \mathrm{~min}$.

\section{Pickering emulsion-based templating}

A 6 wt.\% CNC dispersion was diluted with deionized water to form a 0.25 wt.\% cellulose dispersion. The CNC to PLA ratio in the Pickering emulsion was set to 5 wt.\% to prepare the PLA/CNC Pickering emulsion-based composites. A solution of PLA in DCM (30 ml, $90 \mathrm{mg} / \mathrm{mL})$ was added to the aqueous CNC dispersion (60 $\mathrm{mL})$. $\mathrm{NaCl}(50 \mathrm{mM})$ was added to the aqueous phase to screen the surface charge of the CNC. The mixture was homogenized (IKA T18 homogenizer, Germany) at 12,000 
rpm for 3 min to obtain Pickering emulsions. DCM was evaporated at ambient temperature for $24 \mathrm{~h}$. The precipitates were collected by vacuum filtration using a 500 mesh filter screen. The obtained PLA/CNC microspheres were vacuum dried at $60{ }^{\circ} \mathrm{C}$ for $24 \mathrm{~h}$. PLA/CNC composite films $\left(20 \mathrm{~mm} * 20 \mathrm{~mm} * 1 \mathrm{~mm}, \mathrm{~L}^{*} \mathrm{~W}^{*} \mathrm{H}\right)$ were prepared by hot-pressing the PLA/CNC microspheres in a hot-press (Fontijne, the Netherlands) at $180{ }^{\circ} \mathrm{C}$ and $100 \mathrm{kN}$ for $5 \mathrm{~min}$.

\section{Direct melt blending method}

An aqueous 6 wt.\% CNC dispersion was dried using a freeze-dryer (Freezone 4.5, Labconco, USA) for 2 days. Dry CNC powder and PLA pellets were added to a Miniextruder (DSM) to get PLA/CNC composites with 1, 3, and 5 wt.\% CNC loading. The barrel temperature was set to $190{ }^{\circ} \mathrm{C}$, and the screw speed was $100 \mathrm{rpm}$. PLA/CNC composite films $\left(20 \mathrm{~mm} * 20 \mathrm{~mm} * 1 \mathrm{~mm}, \mathrm{~L}^{*} \mathrm{~W} * \mathrm{H}\right)$ were prepared by hot-pressing employing a hot-press (Fontijne, the Netherlands) at $180{ }^{\circ} \mathrm{C}$ and $100 \mathrm{KN}$ for $5 \mathrm{~min}$.

\subsubsection{Preparation of PLA/CNC composite foams}

The obtained PLA/CNC composite and neat films were saturated with carbon dioxide $\left(\mathrm{CO}_{2}\right)$ (40 bar) in an autoclave for $6 \mathrm{~h}$ at room temperature, followed by rapid depressurization. Subsequently, the $\mathrm{CO}_{2}$ saturated films were foamed for $20 \mathrm{~s}$ by immersing them in a water bath set at $40{ }^{\circ} \mathrm{C}$. Next, the samples were quenched in ice water. Finally, the foams were left to dry in the air for at least $12 \mathrm{~h}$.

\subsubsection{Characterization}

Scanning electron microscopy (SEM) (JSM-6010LA and JSM-7610FPlus, JEOL, Japan) was used to measure CNC dispersion in the PLA matrix and observe the foam morphologies. Before SEM analysis, the samples were freeze fractured following cooling in liquid nitrogen for $5 \mathrm{~min}$. The cross-sections of the composites were coated with a gold-palladium alloy for $40 \mathrm{~s}$. The election acceleration voltage was typically $5 \mathrm{keV}$. The cell size and cell density were detemined by Image J.

Atomic Force Microscopy (AFM) (Bruker, Multimode 8, NanoScope 5 controller) was used to measure the dimensions of CNC. AFM images were collected using the PeakForce tapping mode with a silicon tip (nominal tip apex radius of $8 \mathrm{~nm}$, NanoWorld, Switzerland) utilizing a cantilever of $125 \mu \mathrm{m}$ (NanoWorld, Switzerland) and a resonance frequency of $2 \mathrm{kHz}$. Scanning was performed in the air under ambient conditions $\left(21^{\circ} \mathrm{C}\right.$ and relative humidity $\left.\sim 40 \%\right)$. Image processing was performed using NanoScope software (version 8.15). 
The absorbed $\mathrm{CO}_{2}$ concentration of neat PLA and PLA composites after $6 \mathrm{~h}$ saturation was obtained by measuring the weight of the samples before and after saturation.

Differential scanning calorimetry (DSC) (Perkin Elmer Pyris 1, USA) was used to measure the crystallinity of (CNC) PLA samples after $6 \mathrm{~h} \mathrm{CO} 2$ saturation. Aluminum pans were sealed under air with ca. $5 \mathrm{mg}$ of sample. The samples were heated from 30 to $200{ }^{\circ} \mathrm{C}$, and the heating rate was $10^{\circ} \mathrm{C} / \mathrm{min}$.

The cell density $\left(\mathrm{N}_{v}\right)$ of the foams was calculated by Kumar's theoretical approximation $^{42}$. No direct measurements of cell dimensions over the micrograph are required in this method; only the micrograph area $(A)$ and the total number of cells $(n)$ contained therein should be measured. Together with the magnification factor of the micrograph $(M), \mathrm{N}_{v}$ can be calculated according to equation 1 .

$$
N_{v}=\left[\frac{\left(n M^{2}\right)}{A}\right]^{3 / 2}
$$




\section{Appendix}
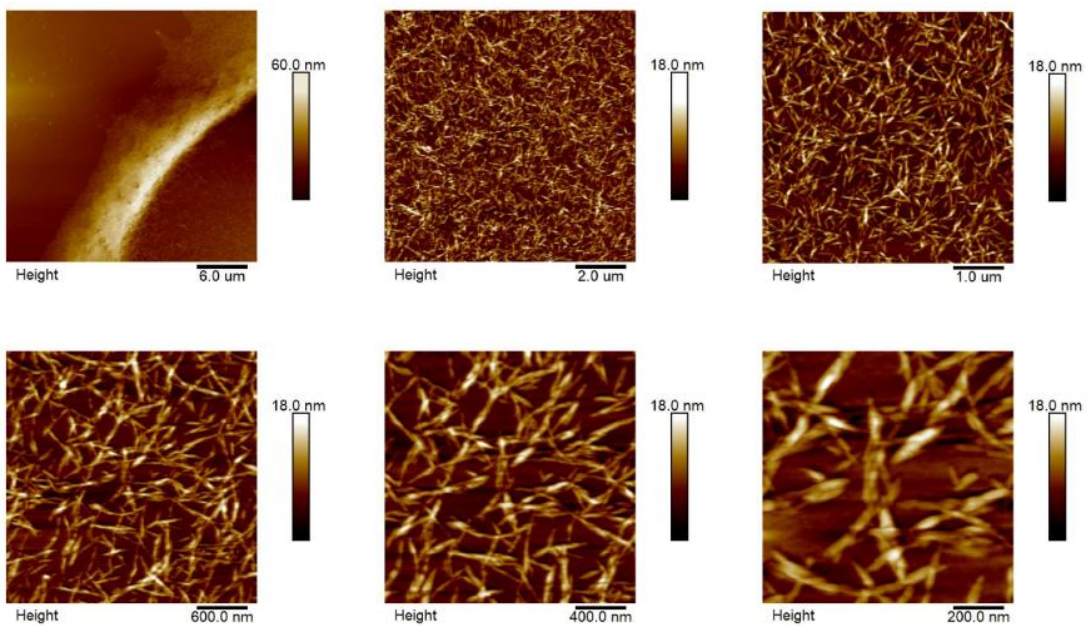

Figure 6-S1 AFM images of CNC deposited on a silicon wafer.

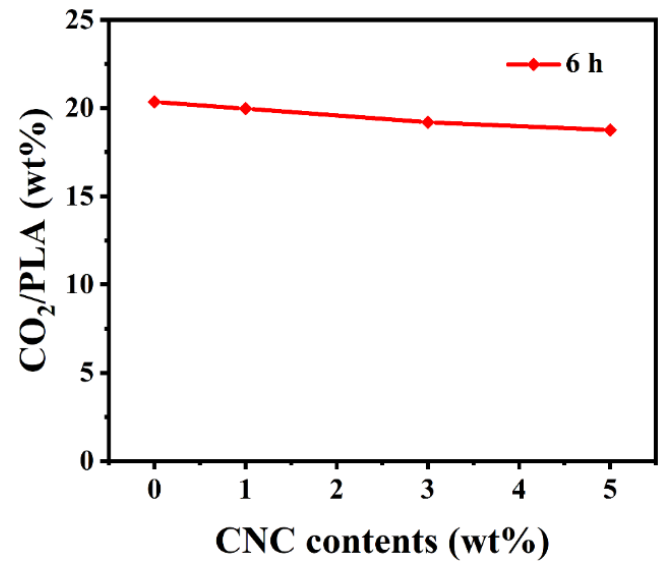

Figure 6-S2 Absorbed $\mathrm{CO}_{2}$ (wt.\%) of composites with a different $\mathrm{CNC}$ loading after $6 \mathrm{~h} \mathrm{CO}_{2}$ saturation at 40 bars. 


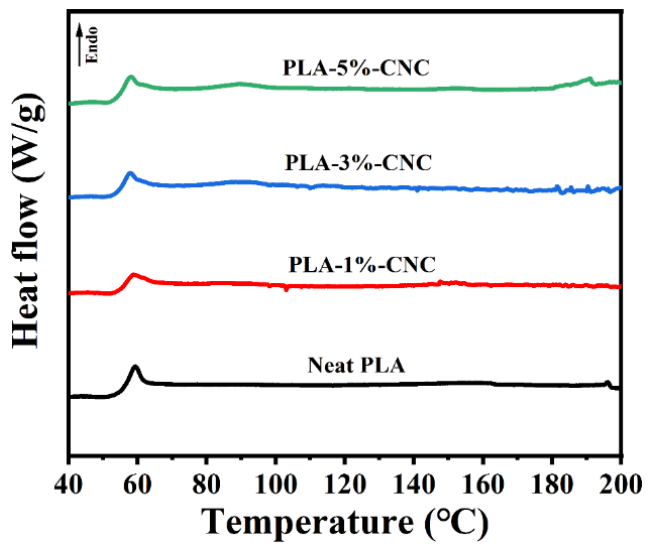

Figure 6-S3 DSC heating traces of composites with different $\mathrm{CNC}$ loadings after $6 \mathrm{~h} \mathrm{CO}_{2}$ saturation at 40 bars. Prior to the DSC measurements the absorbed $\mathrm{CO}_{2}$ was desorbed from the samples at room temperature.
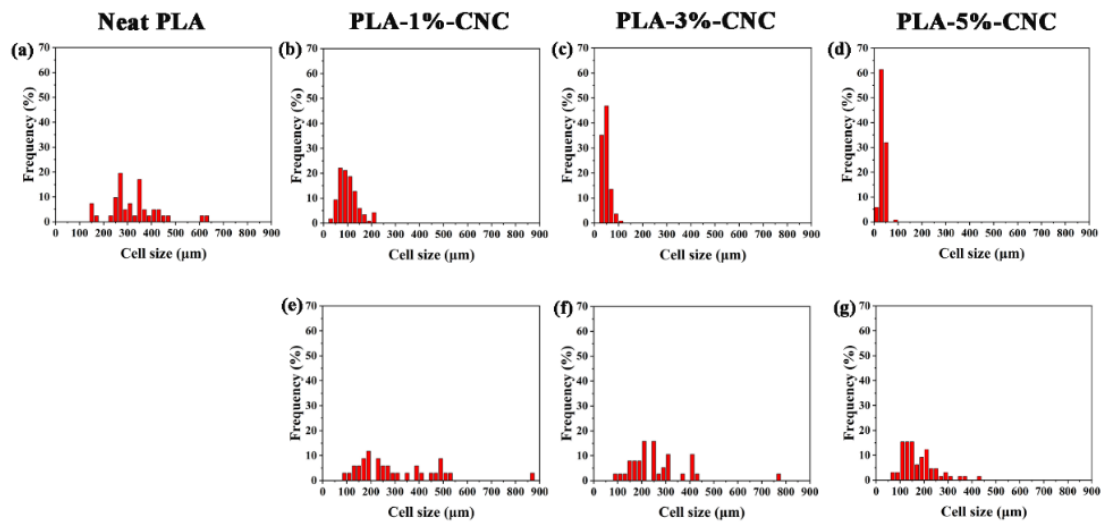

Figure 6-S4 Cell size distribution of PLA/CNC composite foams with different CNC content (up to $5 \mathrm{wt} . \%$ ) prepared by the Pickering emulsion masterbatch route (a-d) and direct melt blending (e-g). 

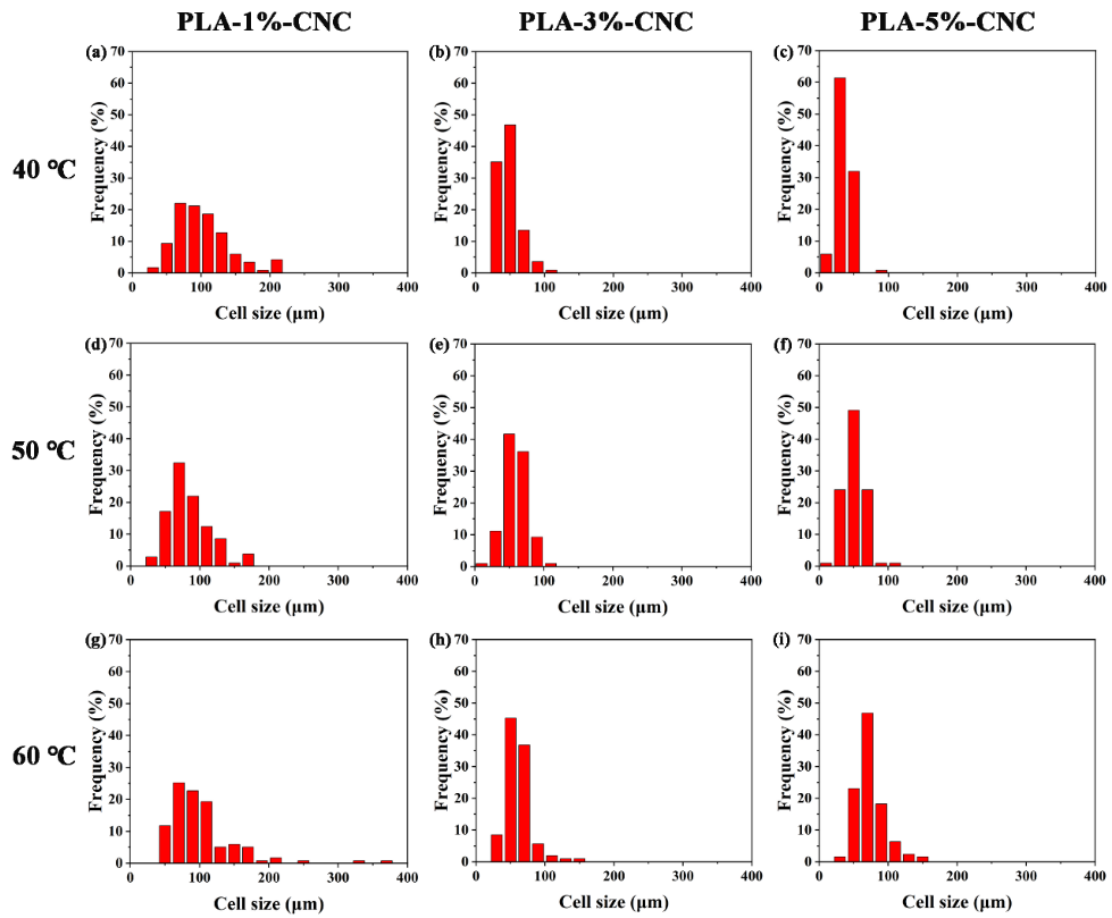

Figure 6-S5 Cell size distribution of PLA/CNC composites foams as a function of the CNC loading and foaming temperature. The $\mathrm{CNC}$ loading was between 0 to $5 \mathrm{wt} . \%$, and the foaming temperature was 40,50 , or $60^{\circ} \mathrm{C}$. 
Foaming of Biodegradable Polylactic Acid/Cellulose Nanocrystal Composites: Pickering Emulsion Templating to Enhance Filler Dispersion and Engineer Foam Morphology
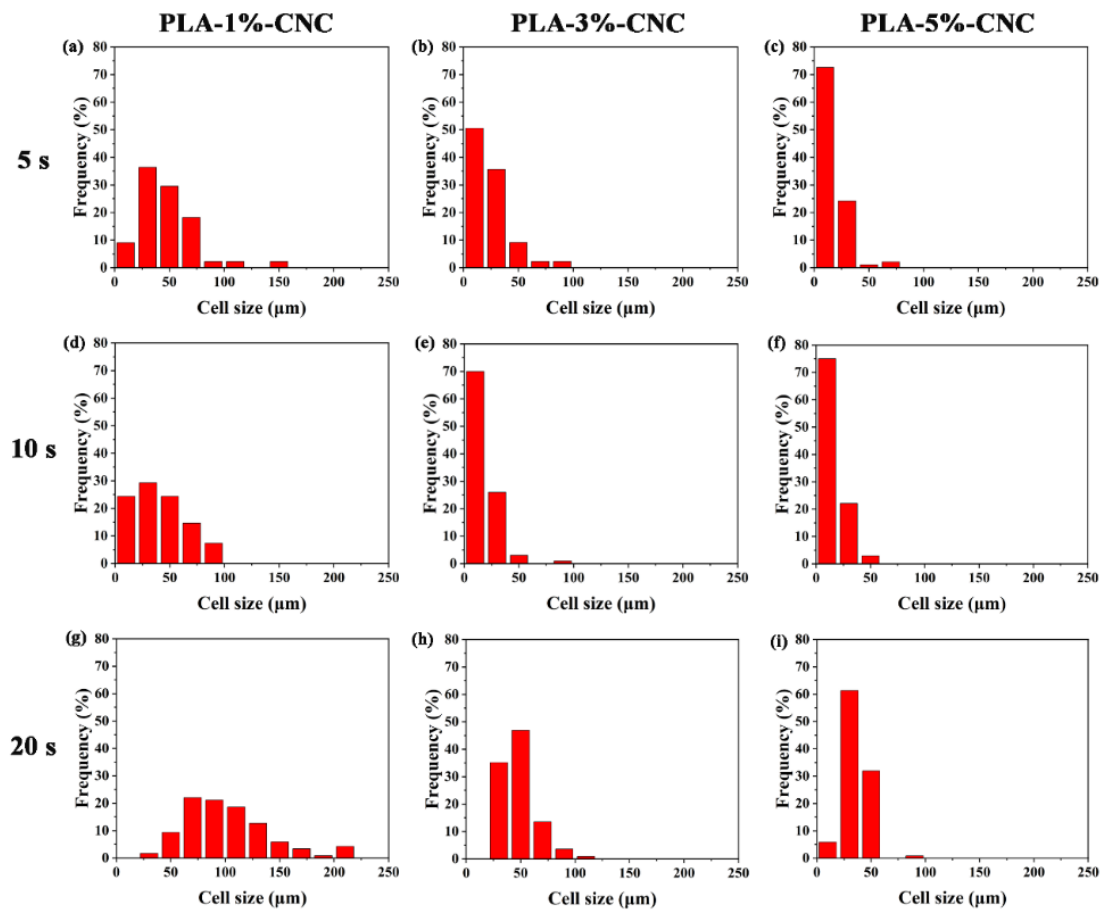

Figure 6-S6 Cell size distribution of PLA/CNC composites foams as a function of the CNC loading and foaming time. The $\mathrm{CNC}$ loading was between 0 to $5 \mathrm{wt} . \%$. The foaming time was 5,10 or 20 seconds. 


\section{References}

[1] Chen, L.; Rende, D.; Schadler, L. S.; Ozisik, R. Polymer nanocomposite foams. Journal of Materials Chemistry A 2013, 1 (12), 3837-3850.

[2] Liu, S.; Duvigneau, J.; Vancso, G. J. Nanocellular polymer foams as promising high performance thermal insulation materials. European Polymer Journal 2015, $65,33-45$.

[3] Abbasi, H.; Antunes, M.; Velasco, J. I. Recent advances in carbon-based polymer nanocomposites for electromagnetic interference shielding. Progress in Materials Science 2019, 103, 319-373.

[4] Jacobs, L. J.; Kemmere, M. F.; Keurentjes, J. T. Sustainable polymer foaming using high pressure carbon dioxide: a review on fundamentals, processes and applications. Green Chemistry 2008, 10 (7), 731-738.

[5] Le Moigne, N.; Sauceau, M.; Benyakhlef, M.; Jemai, R.; Bénézet, J.-C.; Rodier, E.; Lopez-Cuesta, J.-M.; Fages, J. Foaming of poly (3-hydroxybutyrate-co-3hydroxyvalerate)/organo-clays nano-biocomposites by a continuous supercritical CO2 assisted extrusion process. European Polymer Journal 2014, 61, 157-171.

[6] Drumright, R. E.; Gruber, P. R.; Henton, D. E. Polylactic acid technology. Advanced Materials 2000, 12 (23), 1841-1846.

[7] Saeidlou, S.; Huneault, M. A.; Li, H.; Park, C. B. Poly (lactic acid) crystallization. Progress in Polymer Science 2012, 37 (12), 1657-1677.

[8] Nofar, M.; Park, C. B. Poly (lactic acid) foaming. Progress in Polymer Science 2014, 39 (10), 1721-1741.

[9] Kuang, T.-R.; Mi, H.-Y.; Fu, D.-J.; Jing, X.; Chen, B.-y.; Mou, W.-J.; Peng, X.F. Fabrication of poly (lactic acid)/graphene oxide foams with highly oriented and elongated cell structure via unidirectional foaming using supercritical carbon dioxide. Industrial \& Engineering Chemistry Research 2015, 54 (2), 758-768.

[10] Ji, G.; Zhai, W.; Lin, D.; Ren, Q.; Zheng, W.; Jung, D. W. Microcellular foaming of poly (lactic acid)/silica nanocomposites in compressed $\mathrm{CO}_{2}$ : Critical influence of crystallite size on cell morphology and foam expansion. Industrial \& Engineering Chemistry Research 2013, 52 (19), 6390-6398.

[11] Zhao, M.; Ding, X.; Mi, J.; Zhou, H.; Wang, X. Role of high-density polyethylene in the crystallization behaviors, rheological property, and supercritical $\mathrm{CO}_{2}$ 
foaming of poly (lactic acid). Polymer Degradation and Stability 2017, 146, 277 286.

[12] Ren, Q.; Wang, J.; Zhai, W.; Lee, R. E. Fundamental influences of induced crystallization and phase separation on the foaming behavior of poly (lactic acid)/polyethylene glycol blends blown with compressed $\mathrm{CO}_{2}$. Industrial \& Engineering Chemistry Research 2016, 55 (49), 12557-12568.

[13] Mihai, M.; Huneault, M. A.; Favis, B. D. Rheology and extrusion foaming of chain-branched poly (lactic acid). Polymer Engineering \& Science 2010, 50 (3), 629-642.

[14] Ema, Y.; Ikeya, M.; Okamoto, M. Foam processing and cellular structure of polylactide-based nanocomposites. Polymer 2006, 47 (15), 5350-5359.

[15] Keshtkar, M.; Nofar, M.; Park, C. B.; Carreau, P. Extruded PLA/clay nanocomposite foams blown with supercritical $\mathrm{CO}_{2}$. Polymer 2014, 55 (16), 4077-4090.

[16] Li, Y.; Yin, D.; Liu, W.; Zhou, H.; Zhang, Y.; Wang, X. Fabrication of biodegradable poly (lactic acid)/carbon nanotube nanocomposite foams: significant improvement on rheological property and foamability. International Journal of Biological Macromolecules 2020, 163, 1175-1186.

[17] Ding, W.; Kuboki, T.; Wong, A.; Park, C. B.; Sain, M. Rheology, thermal properties, and foaming behavior of high d-content polylactic acid/cellulose nanofiber composites. RSC Advances 2015, 5 (111), 91544-91557.

[18] Dlouhá, J.; Suryanegara, L.; Yano, H. Cellulose nanofibre-poly (lactic acid) microcellular foams exhibiting high tensile toughness. Reactive and Functional Polymers 2014, 85, 201-207.

[19] Dlouhá, J.; Suryanegara, L.; Yano, H. The role of cellulose nanofibres in supercritical foaming of polylactic acid and their effect on the foam morphology. Soft Matter 2012, 8 (33), 8704-8713.

[20] Vatansever, E.; Arslan, D.; Nofar, M. Polylactide cellulose-based nanocomposites. International Journal of Biological Macromolecules 2019, 137, 912-938.

[21] Borkotoky, S. S.; Dhar, P.; Katiyar, V. Biodegradable poly (lactic acid)/cellulose nanocrystals (CNCs) composite microcellular foam: effect of nanofillers on foam cellular morphology, thermal and wettability behavior. International Journal of 
Biological Macromolecules 2018, 106, 433-446.

[22] Lin, N.; Huang, J.; Chang, P. R.; Feng, J.; Yu, J. Surface acetylation of cellulose nanocrystal and its reinforcing function in poly (lactic acid). Carbohydrate Polymers 2011, 83 (4), 1834-1842.

[23] Goffin, A.-L.; Raquez, J.-M.; Duquesne, E.; Siqueira, G.; Habibi, Y.; Dufresne, A.; Dubois, P. From interfacial ring-opening polymerization to melt processing of cellulose nanowhisker-filled polylactide-based nanocomposites. Biomacromolecules 2011, 12 (7), 2456-2465.

[24] Qiu, Y.; Lv, Q.; Wu, D.; Xie, W.; Peng, S.; Lan, R.; Xie, H. Cyclic tensile properties of the polylactide nanocomposite foams containing cellulose nanocrystals. Cellulose 2018, 25 (3), 1795-1807.

[25] Zhang, Y.; Cui, L.; Xu, H.; Feng, X.; Wang, B.; Pukánszky, B.; Mao, Z.; Sui, X. Poly (lactic acid)/cellulose nanocrystal composites via the Pickering emulsion approach: Rheological, thermal and mechanical properties. International Journal of Biological Macromolecules 2019, 137, 197-204.

[26] Zhang, Y.; Jiang, Y.; Han, L.; Wang, B.; Xu, H.; Zhong, Y.; Zhang, L.; Mao, Z.; Sui, X. Biodegradable regenerated cellulose-dispersed composites with improved properties via a Pickering emulsion process. Carbohydrate Polymers 2018, 179, 86-92.

[27] Zhang, Y.; Wu, J.; Wang, B.; Sui, X.; Zhong, Y.; Zhang, L.; Mao, Z.; Xu, H. Cellulose nanofibril-reinforced biodegradable polymer composites obtained via a Pickering emulsion approach. Cellulose 2017, 24 (8), 3313-3322.

[28] Li, X.; Hegyesi, N.; Zhang, Y.; Mao, Z.; Feng, X.; Wang, B.; Pukánszky, B.; Sui, $\mathrm{X}$. Poly (lactic acid)/lignin blends prepared with the Pickering emulsion template method. European Polymer Journal 2019, 110, 378-384.

[29] Kalashnikova, I.; Bizot, H.; Cathala, B.; Capron, I. Modulation of cellulose nanocrystals amphiphilic properties to stabilize oil/water interface. Biomacromolecules 2012, 13 (1), 267-275.

[30] Arias, A.; Heuzey, M.-C.; Huneault, M. A.; Ausias, G.; Bendahou, A. Enhanced dispersion of cellulose nanocrystals in melt-processed polylactide-based nanocomposites. Cellulose 2015, 22 (1), 483-498.

[31] Dhar, P.; Bhasney, S. M.; Kumar, A.; Katiyar, V. Acid functionalized cellulose nanocrystals and its effect on mechanical, thermal, crystallization and surfaces 
properties of poly (lactic acid) bionanocomposites films: A comprehensive study. Polymer 2016, 101, 75-92.

[32] Li, B.; Zhao, G.; Wang, G.; Zhang, L.; Gong, J. Fabrication of high-expansion microcellular PLA foams based on pre-isothermal cold crystallization and supercritical $\mathrm{CO}_{2}$ foaming. Polymer Degradation and Stability 2018, 156, 75-88.

[33] Arslan, D.; Vatansever, E.; Sarul, D. S.; Kahraman, Y.; Gunes, G.; Durmus, A.; Nofar, M. Effect of preparation method on the properties of polylactide/cellulose nanocrystal nanocomposites. Polymer Composites 2020, 41 (10), 4170-4180.

[34] Yin, Y.; Zhao, L.; Jiang, X.; Wang, H.; Gao, W. Cellulose nanocrystals modified with a triazine derivative and their reinforcement of poly (lactic acid)-based bionanocomposites. Cellulose 2018, 25 (5), 2965-2976.

[35] Wang, J.; Chai, J.; Wang, G.; Zhao, J.; Zhang, D.; Li, B.; Zhao, H.; Zhao, G. Strong and thermally insulating polylactic acid/glass fiber composite foam fabricated by supercritical carbon dioxide foaming. International Journal of Biological Macromolecules 2019, 138, 144-155.

[36] Zheng, T.; Pilla, S. Melt processing of cellulose nanocrystal-filled composites: toward reinforcement and foam nucleation. Industrial \& Engineering Chemistry Research 2020, 59 (18), 8511-8531.

[37] Boissard, C. I.; Bourban, P.-E.; Plummer, C. J. G.; Neagu, R. C.; Månson, J.-A. E. Cellular biocomposites from polylactide and microfibrillated cellulose. Journal of Cellular Plastics 2012, 48 (5), 445-458.

[38] Zimmermann, M. V.; da Silva, M. P.; Zattera, A. J.; Santana, R. M. Poly (lactic acid) foams reinforced with cellulose micro and nanofibers and foamed by chemical blowing agents. Journal of Cellular Plastics 2018, 54 (3), 577-596.

[39] Cho, S. Y.; Park, H. H.; Yun, Y. S.; Jin, H.-J. Influence of cellulose nanofibers on the morphology and physical properties of poly (lactic acid) foaming by supercritical carbon dioxide. Macromolecular Research 2013, 21 (5), 529-533.

[40] Kang, D. J.; Xu, D.; Zhang, Z. X.; Pal, K.; Bang, D. S.; Kim, J. K. Well-controlled microcellular biodegradable PLA/silk composite foams using supercritical $\mathrm{CO}_{2}$. Macromolecular Materials and Engineering 2009, 294 (9), 620-624.

[41] Matuana, L.; Faruk, O. Effect of gas saturation conditions on the expansion ratio of microcellular poly (lactic acid)/wood-flour composites. Express Polymer Letters 2010, 4 (10), 621-631. 
[42] Kumar, V.; Suh, N. P. A process for making microcellular thermoplastic parts. Polymer Engineering \& Science 1990, 30 (20), 1323-1329. 


\section{Chapter 7}

Highly Stable and Non-Flammable Hydrated Salt-Paraffin ShapeMemory Gels for Sustainable Building Technology

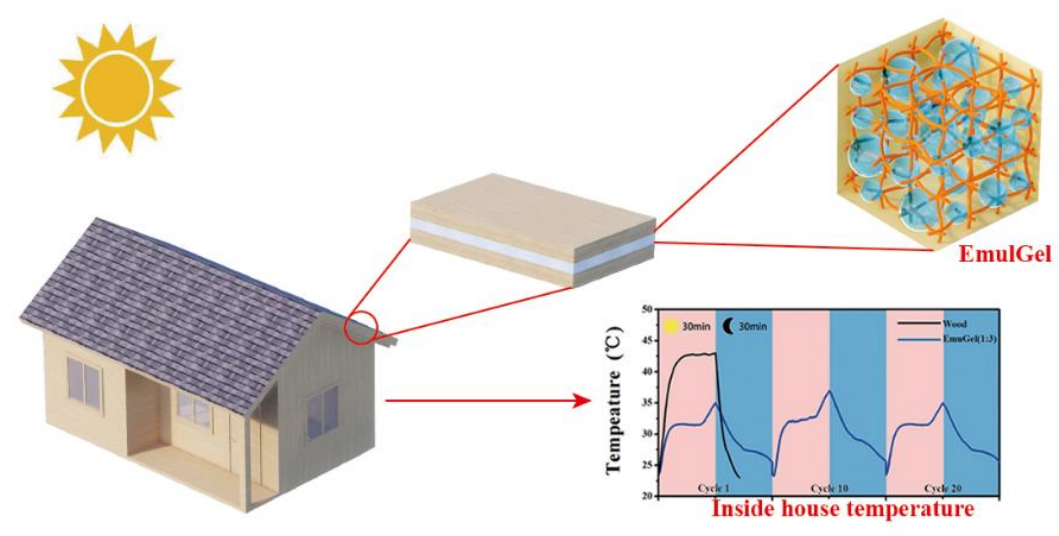


Hydrated salts (salt hydrates) are highly promising low-temperature phase change materials (PCM) due to their high cohesive energy density and low cost. However, they exhibit phase separation, liquid leakage, and inherent supercooling, which hinders their applications in sustainable building technology. Here, we describe the design of a highly stable emulsion gel system (EmulGels) that exhibits non-flammable and shape-memory characteristics. Oleophilic paraffin and hydrophilic hydrated salts, both are excellent PCMs typically existing in separate phases, are combined harmoniously in a hydrogel by a templating water-in-oil Pickering emulsion. Latent heat values of the prepared EmulGels were up to $213.2 \mathrm{~J} / \mathrm{g}$ (eicosane/disodium hydrogen phosphate dodecahydrate $=1: 3$ ). No leakage of eicosane was noticed after heating the EmulGels at $60{ }^{\circ} \mathrm{C}$ for $30 \mathrm{~min}$, and the latent heat value remained almost unchanged following 500 thermal cycles. The EmulGel was specifically designed to enable dual-phase crosslinking, which effectively enhanced its shape-stability, prevented loss of water of crystallization in hydrated salts, and decreased the degree of supercooling. Non-flammable characteristics typically found in hydrated salts was also exhibited by the EmulGel, in combination with good mechanical properties. The materials characteristics make EmulGels ideal candidates to serve as building construction interlayers for effective thermal building management.

\footnotetext{
*The contents of this chapter have been submitted (under review) in: Yunchong Zhang, Feifei Wang, Joost Duvigneau, Yan Wang, Bijia Wang, Xueling Feng, Zhiping Mao, G. Julius Vancso and Xiaofeng Sui; Highly Stable and Non-Flammable Hydrated Salt-Paraffin Shape-Memory Gels for Sustainable Building Technology.
} 


\subsection{Introduction}

It is estimated that $70 \%$ of the world's population will be living in an urban environment by $2050^{1}$. The rapid increase in urban population has been accompanied by a continuous increase in building use related energy consumption. In many countries, energy consumption by the residential sector could be up to $40 \%$ of the total energy demand, of which over half is used for space heating ${ }^{2-3}$. From the perspective of urban sustainable development needs, balanced building energy management is essential ${ }^{4}$. In order to reduce the energy consumption of buildings, passive energysaving technologies have been extensively explored ${ }^{5-6}$.

Phase change materials (PCMs) are excellent thermal energy storage substances that take in, or release, high amounts of latent heat during melting or crystallization processes. This heat can be utilized to provide useful heating/cooling around the corresponding phase change temperatures ${ }^{7-12}$. Based on their chemical composition, PCMs generally can be divided into organic (paraffin, fatty acids, alcohols, etc.) and inorganic (hydrated salts, metallics, etc.) materials classes ${ }^{13}$. PCMs can play an essential role in passive energy management of buildings by reducing the mismatch between energy supply and demand ${ }^{14-15}$. Paraffin is one of the most frequently used PCM constituents due to its suitable melting temperatures, negligible supercooling effects, and chemical stability ${ }^{16}$. For example, hollow floor panels filled with 85 wt.\% paraffin reported by Royon and coworkers ${ }^{17}$ could effectively lower the surface wall temperatures of buildings by storing solar energy during the day. Unfortunately, the application of paraffin in buildings is limited by its high flammability and high cost. On the other hand, hydrated salts, due to their high latent heat of fusion, nonflammability, high thermal conductivity, low volume changes, and low $\operatorname{cost}^{18-21}$, make these materials highly interesting candidates for building thermal management. For example, Zhang and coworkers ${ }^{22}$ impregnated eutectic hydrated salts $\left(\mathrm{Na}_{2} \mathrm{SO}_{4} \cdot 10 \mathrm{H}_{2} \mathrm{O}-\mathrm{Na}_{2} \mathrm{CO}_{3} \cdot 10 \mathrm{H}_{2} \mathrm{O}\right.$ with $1: 1$ in mass ratio) into expanded vermiculite used for building energy storage. The maximum temperature of the room tempered by these systems was $4.8^{\circ} \mathrm{C}$ lower than that of the control chamber.

However, hydrated salts could be highly corrosive when leaked, and their performance may suffer from supercooling effects, which significantly limits their broader application. If progress is to be made with these phase change materials, these shortcomings must be addressed ${ }^{23}$. In addition to corrosion, leakage of hydrated salts will cause loss of water. Once the water from the crystallizable phase separates or vaporizes during the phase change process, the hydrated salt transforms into a 
dehydrated form that melts at much higher temperatures. Immobilization of PCMs is an excellent way to solve the problem of leakage and thus to prevent water escape, by using porous materials as support ${ }^{20}$, or encapsulation of the PCMs within micro- or nano-scaled shells ${ }^{24-25}$. For example, eutectic hydrated salts $\left(\mathrm{Na}_{2} \mathrm{SO}_{4} \cdot 10 \mathrm{H}_{2} \mathrm{O}-\right.$ $\mathrm{Na}_{2} \mathrm{CO}_{3} \cdot 10 \mathrm{H}_{2} \mathrm{O}$ ) were fixed in poly(acrylamide-co-acrylic acid) copolymer hydrogels by Liu and coworkers ${ }^{26}$ to prevent water loss of the hydrated salts. The latent heat of PCM hydrogels remained unchanged following 300 cycles in a thermocycling test chamber in the reported experiments. However, specific water loss of the hydrogel was not considered. Shchukin and coworkers ${ }^{27}$ encapsulated $\mathrm{Mg}\left(\mathrm{NO}_{3}\right)_{2} \cdot 6 \mathrm{H}_{2} \mathrm{O}$ within silica nano-scaled shells via Pickering emulsion templating. The PCM nanocapsules obtained significantly improved thermal cyclability. These materials, however, still exhibited 32 wt.\% water loss after 100 cycles, and supercooling remained problematic. Controlling the degree of supercooling is another challenge that remains to be tackled since it defines the effective temperature range of use of a particular hydrated salt. The common practice to decrease the degree of supercooling of hydrated salts involves adding nucleating agents such as $\mathrm{SiO}_{2}{ }^{28}, \mathrm{CaO}^{29}, \mathrm{Mg}(\mathrm{OH})_{2}{ }^{30}, \mathrm{CaCO}_{3}{ }^{31}$, or $\mathrm{KCl}^{32}$ to promote crystallization. It is worth mentioning that the common organic PCM-paraffin materials can also function as an effective nucleating agent, as demonstrated by Li and coworkers ${ }^{33}$ and our group ${ }^{34}$.

In this work, we propose to address both the supercooling and the dehydration problems of hydrated salts used as PCMs, by combining two phase change materials via a water-in-oil Pickering emulsion templating method. The resulting hybrid gel (EmulGel), supported by an in situ polymerized copolymer, consisting of disodium hydrogen phosphate dodecahydrate (DHPD) and eicosane, which have similar melting temperatures and, when combined, endow corresponding gels with a high latent heat storage capacity. This designer hybrid gel provides a dual protection mechanism for the loss water of crystallization of DHPD. The hydrophilic sodium polyacrylate chain polymerized in the aqueous phase can effectively prevent water loss. Additionally, the eicosane effectively minimizes the loss of water from DHPD as a sealant, and it also helps to decrease the degree of supercooling of DHPD by acting as a heterogeneous nucleating agent. The favorable interaction between the crosslinked PMMA-DMA polymer network and eicosane effectively prevents leakage above melting temperatures. The high potential of our hybrid gel for use in building thermal management was demonstrated by a "model house", equipped with a gelloaded roof. An added feature of the hybrid gel that enhances suitability for building 
applications is its non-flammability owing to the non-flammable nature of DHPD.

\subsection{Results and Discussion}

\subsubsection{The formation of EmulGels}

We first describe the preparation and structural characterization of the EmulGels. A schematic of the structure is depicted in Figure 7.1a. Before polymerization, the hydrophilic monomer, NaAA, would preferentially distribute in the inner aqueous phase, while the hydrophobic MMA and DMA tended to stay in the oily phase. Upon heating to $70{ }^{\circ} \mathrm{C}$, dual-phase polymerization of the acrylates led to gelation of the emulsion to afford the hybrid emulsion gel. Hydrophobic fumed silica was used as emulsifier for the Pickering emulsion, making the system more stable due to the Pickering effect ${ }^{35-38}$. Images of the Pickering emulsion and EmulGel (1:3) are shown in Figure 7.1b and 7.1e, respectively. The optical micrograph in Figure 7.1c display that the w/o Pickering emulsion consists of evenly distributed molten hydrated salt droplets of about $1 \mu \mathrm{m}$ in diameter, whose outline was still recognizable in the SEM image of the EmulGel shown in Figure 7.1d. However, the spherical boundary of the templated emulsion droplets was partially lost upon formation of the EmulGel due to crystallization of the incorporated phase change components. 
(a)
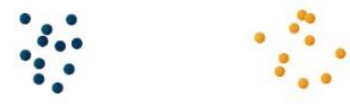

NaAA

MMA and DMA

DHPD

Eicosane
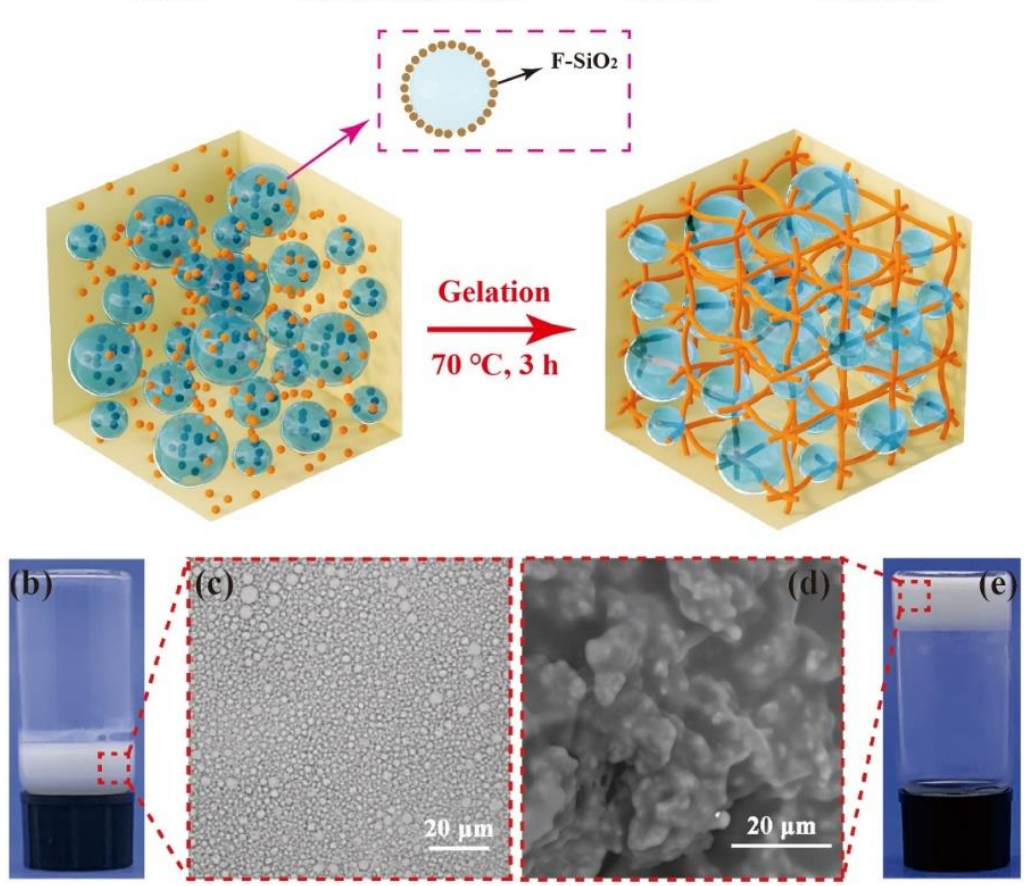

Figure 7.1 (a) Schematic of the structure and the preparation steps of EmulGels obtained via Pickering emulsion templating. (b) Photograph of the Pickering emulsion. (c) Optical micrograph of the w/o Pickering emulsion. (d) SEM image of EmulGel (1:3) and (e) Photograph of EmulGel (1:3).

Optical micrographs of emulsions with different DHPD : Eicosane ratios are shown in Figure 7-S(1-2). An increase in the DHPD content would lead to the formation of larger emulsion droplets. Hence it would have a negative effect on the stability of the emulsions. For this reason, we chose an eicosane to DHPD mass ratio of 1:3 for further investigations, albeit we note that all emulsions were reasonably stable during the polymerization process, as manifested by the lack of delamination following storage at $70{ }^{\circ} \mathrm{C}$ for three hours (see Figure 7-S3).

\subsubsection{The stability of EmulGels}

We shall now discuss the thermal transitions of our gels. The phase change behavior of EmulGel (1:3) was investigated using DSC and the characteristics were 
compared to DHPD and eicosane. As is shown in Figure 7.2a, DHPD and eicosane have essentially overlapping melting peaks, but they exhibit a notably different crystallization behavior. DHPD crystalizes at a temperature around $7^{\circ} \mathrm{C}$, significantly lower than its $\mathrm{T}_{\mathrm{m}}$ due to supercooling, which would limit its application as a PCM. The DSC trace of the EmulGel (1:3) showed one melting peak.

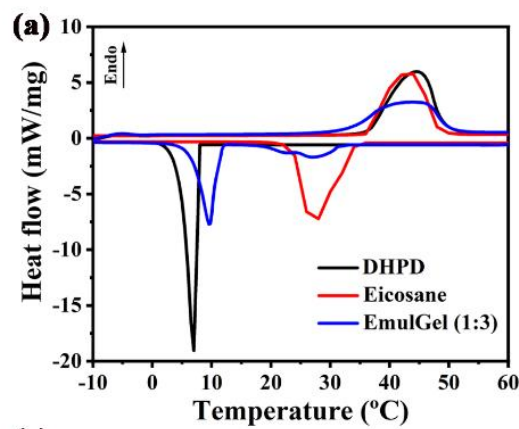

(b)
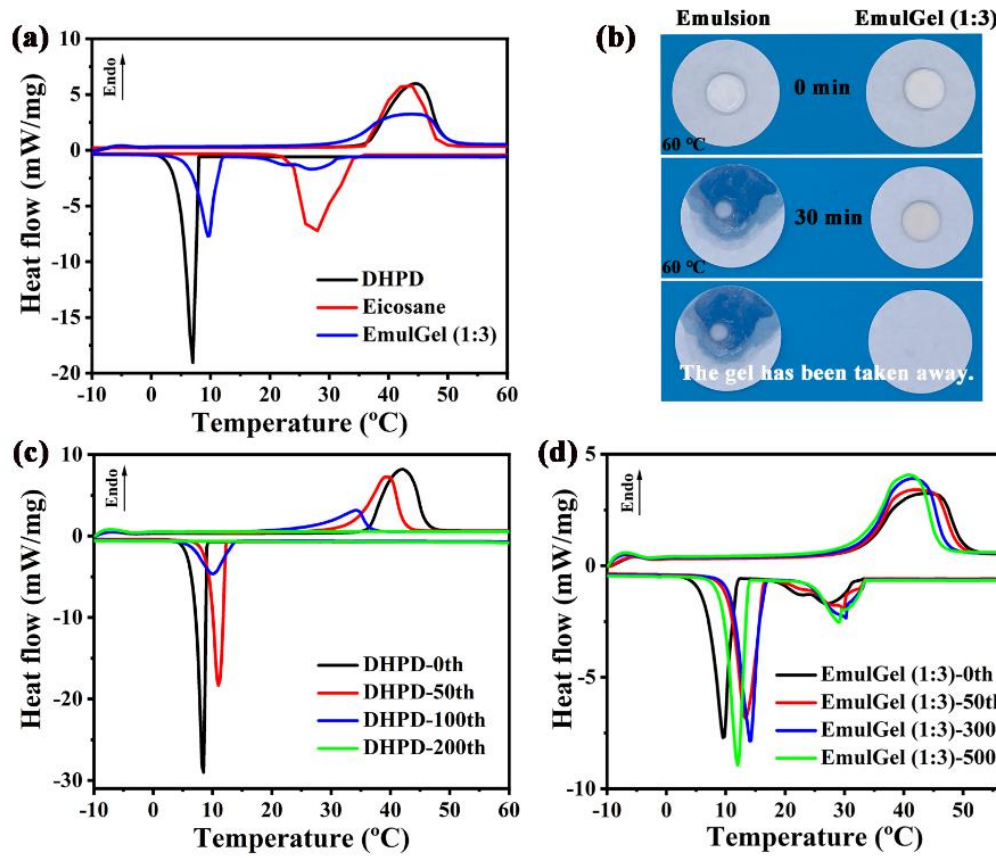

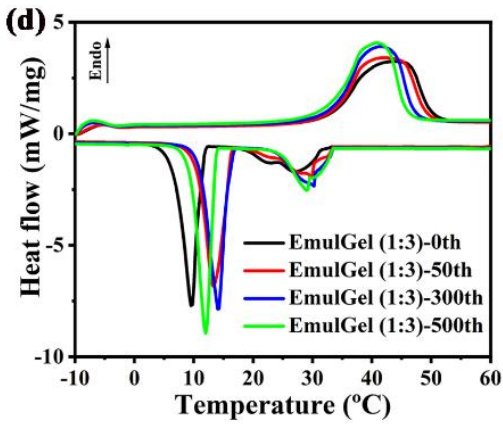

Figure 7.2 (a) DSC traces of DHPD, eicosane, and EmulGel (1:3). (b) Leakage proof properties of EmulGel (1:3). (c) DSC traces of cycling test of DHPD and (d) DSC traces of cycling test of EmulGel (1:3).

The latent heat values of DHPD and eicosane in EmulGel (1:3) were $129.3 \mathrm{~J} / \mathrm{g}$ and $55.8 \mathrm{~J} / \mathrm{g}$, which corresponds to an encapsulation ratio (see experimental section and Table 7-S1) of $56 \%$ and $22 \%$, respectively. The overall latent heat value and encapsulation ratio for the EmulGel (1:3) were $213.2 \mathrm{~J} / \mathrm{g}$ and $78 \%$, respectively. The thermal performance of phase change gels in the present and previous studies is summarized and shown in Table 7.1. The heat capacity of EmulGel (1:3) compares favorably with similar shape stable PCM composites. 
Table 7.1. Comparison of the thermal performance of phase change gels investigated in the present and previous studies.

\begin{tabular}{|c|c|c|c|c|c|}
\hline Year & PCM & Gel type & $\begin{array}{l}\text { Monomers or } \\
\text { polymer }\end{array}$ & $\begin{array}{l}\text { Latent } \\
\text { heat }(\mathrm{J} / \mathrm{g})\end{array}$ & Ref \\
\hline $\begin{array}{l}\text { This } \\
\text { work }\end{array}$ & $\begin{array}{l}\mathrm{Na}_{2} \mathrm{HPO}_{4} \cdot 12 \mathrm{H}_{2} \mathrm{O}, \\
\text { Eicosane }\end{array}$ & $\begin{array}{l}\text { Emulsion } \\
\text { gel }\end{array}$ & $\begin{array}{l}\text { Aqueous phase: } \\
\text { MMA and DMA } \\
\text { Oil phase: NaAA }\end{array}$ & $\begin{array}{l}213.2 \\
(\text { EmulGel } \\
(1: 3))\end{array}$ & $\begin{array}{l}\text { This } \\
\text { work }\end{array}$ \\
\hline 2018 & $\mathrm{NaAc} \cdot 3 \mathrm{H}_{2} \mathrm{O}$ & Hydrogel & $\begin{array}{l}\text { Acrylamideacrylic } \\
\text { acid sodium co- } \\
\text { polymer }\end{array}$ & $\begin{array}{l}202.4 \\
(\mathrm{LTPCH})\end{array}$ & 39 \\
\hline 2016 & $\mathrm{Na}_{2} \mathrm{SO}_{4} \cdot 10 \mathrm{H}_{2} \mathrm{O}$ & Hydrogel & $\begin{array}{l}\text { Sodium alginate; } \\
\text { Acrylamide }\end{array}$ & $202.9(5)$ & 40 \\
\hline 2016 & $\begin{array}{l}\mathrm{Na}_{2} \mathrm{HPO}_{4} \cdot 12 \mathrm{H}_{2} \mathrm{O} \\
\mathrm{Na}_{2} \mathrm{CO}_{3} \cdot 10 \mathrm{H}_{2} \mathrm{O}\end{array}$ & Hydrogel & $\begin{array}{l}\text { Acrylic acid; } \\
\text { Acrylamide }\end{array}$ & $\begin{array}{l}189.1 \\
(\mathrm{EHS} / \mathrm{PAA} \\
\text { AM) }\end{array}$ & 26 \\
\hline 2020 & $\mathrm{Na}_{2} \mathrm{SO}_{4} \cdot 10 \mathrm{H}_{2} \mathrm{O}$ & Hydrogel & $\begin{array}{l}\text { Poly (acrylate } \\
\text { sodium) copolymer }\end{array}$ & $\begin{array}{l}187.2 \\
\text { (MWCNT- } \\
\text { GS/PAAS) }\end{array}$ & 41 \\
\hline 2018 & Octadecane & $\begin{array}{l}\text { Closed- } \\
\text { cell } \\
\text { hydrogel }\end{array}$ & $\mathrm{NaAA}$ & $\begin{array}{l}161.9 \text { (HG- } \\
1-\mathrm{D}-\mathrm{OD})\end{array}$ & 42 \\
\hline 2017 & PEG & Hydrogel & PEG & $\begin{array}{l}154.2 \\
(\text { PEGH-8 } \\
(25.5))\end{array}$ & 43 \\
\hline
\end{tabular}

As a composite shape-stable PCM, the EmulGel also showed good leakage-proof properties (Figure 7.2b), as it could be heated to $60^{\circ} \mathrm{C}$ for $30 \mathrm{~min}$ and still maintained its original shape and firmly held the molten DHPD and eicosane. In comparison, serious leakage was observed for a control emulsion sample that was not polymerized. This drastic difference demonstrates that oil-phase polymerization effectively restricts the movement of eicosane and provides an excellent oil seal to the inner DHPD core. We note that the core is further restricted by the polymerization of sodium acrylate. 
The leakage-proof properties of Eicosane-Gel are illustrated in Figure 7-S4. There is still no stain of leaked eicosane on the filter paper observed, indicating the strong eicosane absorption ability of polymer chains in the oily phase.

A remarkable improvement in cyclability was achieved by the dual phase polymerization strategy. Thermal cycling tests were performed on EmulGel (1:3) and DHPD was used as control. The results are summarized in Figure 7.2c-d and Table 7.2. Compared with the control DHPD, which completely lost its melting enthalpy after 200 cycles, EmulGel (1:3) maintained 98\% of its original melting enthalpy after 500 cycles.

Table 7.2 Thermal cyclability of DHPD and EmulGel (1:3).

\begin{tabular}{lccc}
\hline Sample & $\Delta \mathrm{H}_{\mathrm{m}}(\mathrm{J} / \mathrm{g})$ & $\Delta \mathrm{H}_{\mathrm{c}(\mathrm{DHPD})}(\mathrm{J} / \mathrm{g})$ & $\Delta \mathrm{H}_{\mathrm{c} \text { (Eicosane })}(\mathrm{J} / \mathrm{g})$ \\
\hline DHPD-0th & 278.0 & 229.9 & - \\
DHPD-50th & 248.6 & 210.3 & - \\
DHPD-100th & 126.5 & 108.9 & - \\
DHPD-200th & 0 & 0 & - \\
EmulGel (1:3)-0th & 213.2 & 129.3 & 55.8 \\
EmulGel (1:3)-50th & 217.5 & 132.8 & 53.6 \\
EmulGel (1:3)-300th & 216.3 & 133.1 & 56.2 \\
EmulGel (1:3)-500th & 207.9 & 127.5 & 55.4 \\
\hline
\end{tabular}

\subsubsection{Water retention ability and degree of supercooling of EmulGels}

The schematic of the phase change process of EmulGels is shown in Figure 7.3a. The molten aqueous phase remained confined inside the gel due to the efficient sealing by the internal hydrophilic polymer network and eicosane acting as a powerful sealant. 
(a)

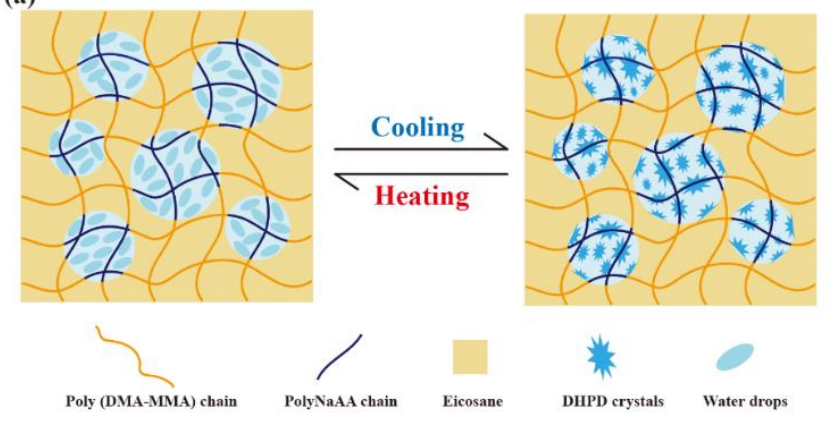

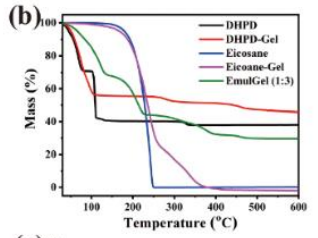

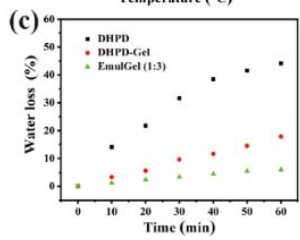

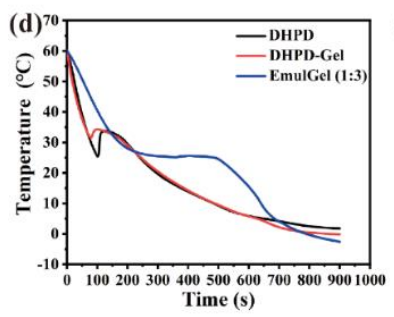
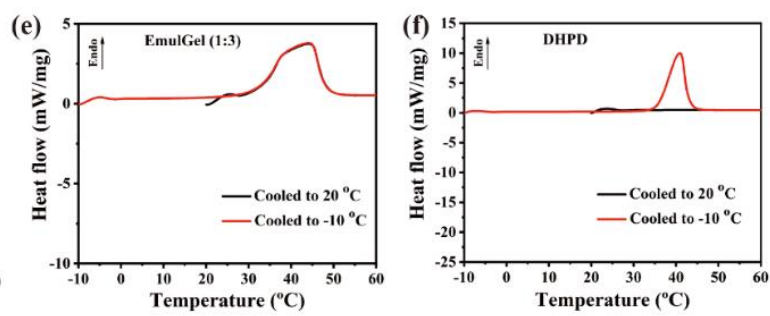

Figure 7.3 (a) Schematic of the phase change transition process of EmulGels. (b) TGA curves of DHPD, DHPD-Gel, eicosane, Eicosane-Gel, and EmulGel (1:3). (c) Loss of water of DHPD, DHPD-Gel, and EmulGel (1:3) at $60{ }^{\circ} \mathrm{C}$. (d) Cooling curves of DHPD, DHPD-Gel, and EmulGel (1:3). DSC traces of (e) melting EmulGel (1:3) and (f) melting of DHPD, which were cooled to predetermined temperatures and kept for $5 \mathrm{~min}$ at that temperature (black line: $20^{\circ} \mathrm{C}$; red line: $\left.-10^{\circ} \mathrm{C}\right)$.

The strong water retention ability of EmulGel (1:3) was confirmed using TGA (Figure 7.3b) and water loss tests (Figure 7.3c). As shown by the TGA curves of DHPD, DHPD-Gel, and EmulGel (1:3), the internal hydrophilic polymer network was less efficient than the sealing effect of eicosane in preventing dehydration of DHPD. By comparing the TGA curves of eicosane and Eicosane-Gel, it becomes clear that the oil phase polymerization of MMA and DMA had little stabilization effect on eicosane. The second mass loss (from around $250{ }^{\circ} \mathrm{C}$ to around $390{ }^{\circ} \mathrm{C}$ ) on the eicosane-Gel curve is likely due to the degradation of the PMMA-DMA polymer network.

The results of the water-loss tests are shown in Figure 7.3c. The $\Delta H_{m}$ values of the samples after the water loss tests were measured and are shown in Figure 7-S5 and summarized in Table 7-S2. The $\Delta \mathrm{H}_{\mathrm{m}}$ value of DHPD decreased by $49 \%$ after being heated at $60{ }^{\circ} \mathrm{C}$ for $1 \mathrm{~h}$, corresponding to a loss of $44 \%$ water of crystallization. 
The DHPD-Gel had improved water retaining capacity and lost $18 \%$ water of crystallization after $1 \mathrm{~h}$, while EmulGel (1:3) lost only $5.9 \%$ of its water of crystallization.

Supercooling is another known main problem for practical applications of hydrated salts. The cooling curves of EmulGel (1:3) are compared to those of DHPD and DHPD-Gel in Figure 7.3d. It is shown that supercooling of DHPD was reduced from $8.3^{\circ} \mathrm{C}$ to $2.8^{\circ} \mathrm{C}$ in DHPD-Gel which is attributed to the thickening effect of the sodium polyacrylate crosslinking network. Further improvement was achieved with EmulGel (1:3), where supercooling was eliminated, possibly due to the heterogeneous nucleation effect of Span80, $\mathrm{F}_{-}-\mathrm{SiO}_{2}$, or eicosane ${ }^{44-45}$. The decrease in degree of supercooling is also confirmed by the fact that the DHPD in EmulGel (1:3) readily crystallized when cooled from its melt to $20{ }^{\circ} \mathrm{C}$ and that it showed a nearly identical melting peak compared to when the sample was cooled to $-10^{\circ} \mathrm{C}$ (Figure 7.3e). Pure DHPD did not show any melting peak when reheated from $20{ }^{\circ} \mathrm{C}$ (see Figure 7.3f). We note that this improvement would significantly broaden the applicability of this composite PCM.

\subsubsection{Mechanical properties of EmulGels}

Good mechanical properties are essential for PCM targeted at applications in the construction industry. Compressibility values of EmulGels with different eicosane to DHPD mass ratios $(1: 2.5,1: 3,1: 3.5,1: 4,1: 4.5)$ were obtained at room temperature (Figure 7.4a-b). The EmulGels showed steadily increasing compression strength and modulus with increasing DHPD contents. Good compression modulus values of up to 20.6 MPa could be achieved in EmulGel (1:4.5), corresponding to an enhancement by $430 \%$ compared the $3.9 \mathrm{MPa}$ of EmulGel (1:2.5). The enhancement in compression strength with increasing DHPD contents could be attributed to the superior strength of the inner phase crystallohydrate-polymer composites with compact crystal columns arranged in parallel fashion ${ }^{46}$. 

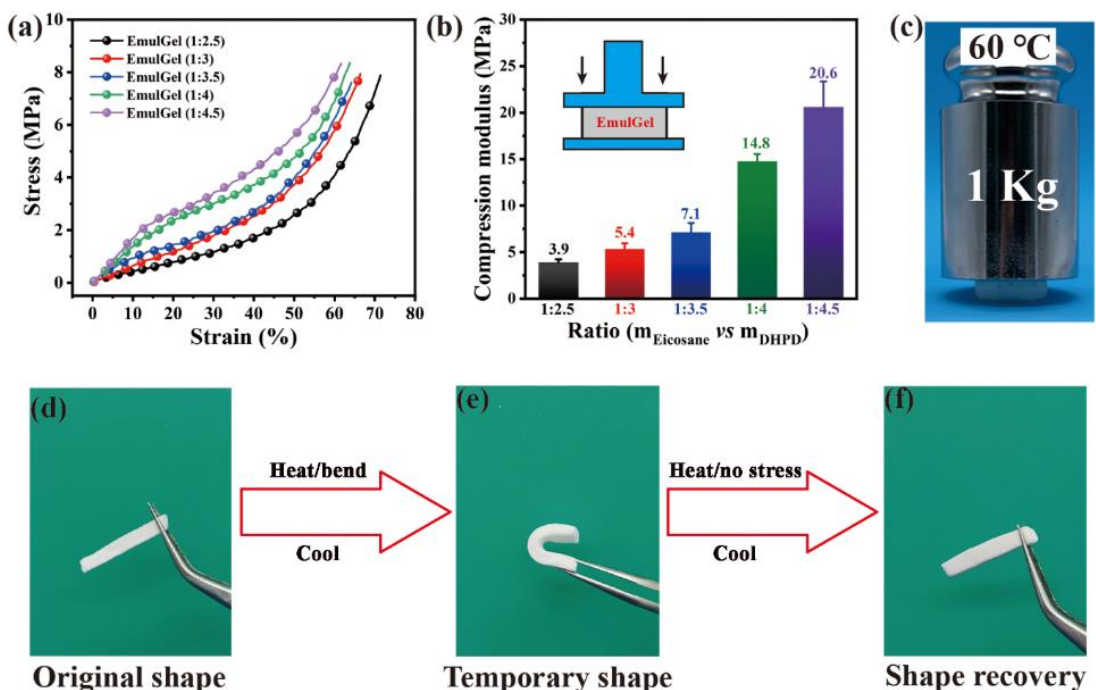

Shape recovery

Figure 7.4 (a) Compressive stress-strain curves and (b) compression modulus of the EmulGels with different eicosane to DHPD mass ratios. (c) Photograph showing mechanical behavior under a compressive load for a molten EmulGel (1:3). (d-f) Photographs displaying shape memory characteristics of EmulGel (1:3).

As is shown in Figure 7.4c, the EmulGel (1:3) was strong enough to hold a load of $1 \mathrm{~kg}$ weight when heated to above the $\mathrm{T}_{\mathrm{m}}$ of eicosane and DHPD $\left(60^{\circ} \mathrm{C}\right)$. The EmulGel (1:3) also displayed good shape memory behavior empowered by the phase change behavior of eicosane and DHPD. As demonstrated in Figure 7.4d-f, strips of the gel could be heated and fixed to temporary $U$ shapes, and they fully recover to their original shape when reheated without applying external stress. This is contributed to the phase transition ability of PCMs. We note that similar malleability of shape stable PCM composites was also reported by Liu and coworkers ${ }^{47-48}$.

\subsubsection{Building thermal management by EmulGels}

The utilization of EmulGels as a promising thermal management system for buildings was demonstrated by sandwiching gels between two wooden planks (Figure 7.5a-b) used as a "roof" in a wooden model house. The schematic of model house test setup is shown in Figure 7.5c. A xenon lamp was used to simulate sunlight and the model house was subjected to "on" and "off" irradiation for 30 min intermittently. A second wooden model house with a regular wooden roof of the same thickness was used as the control. 


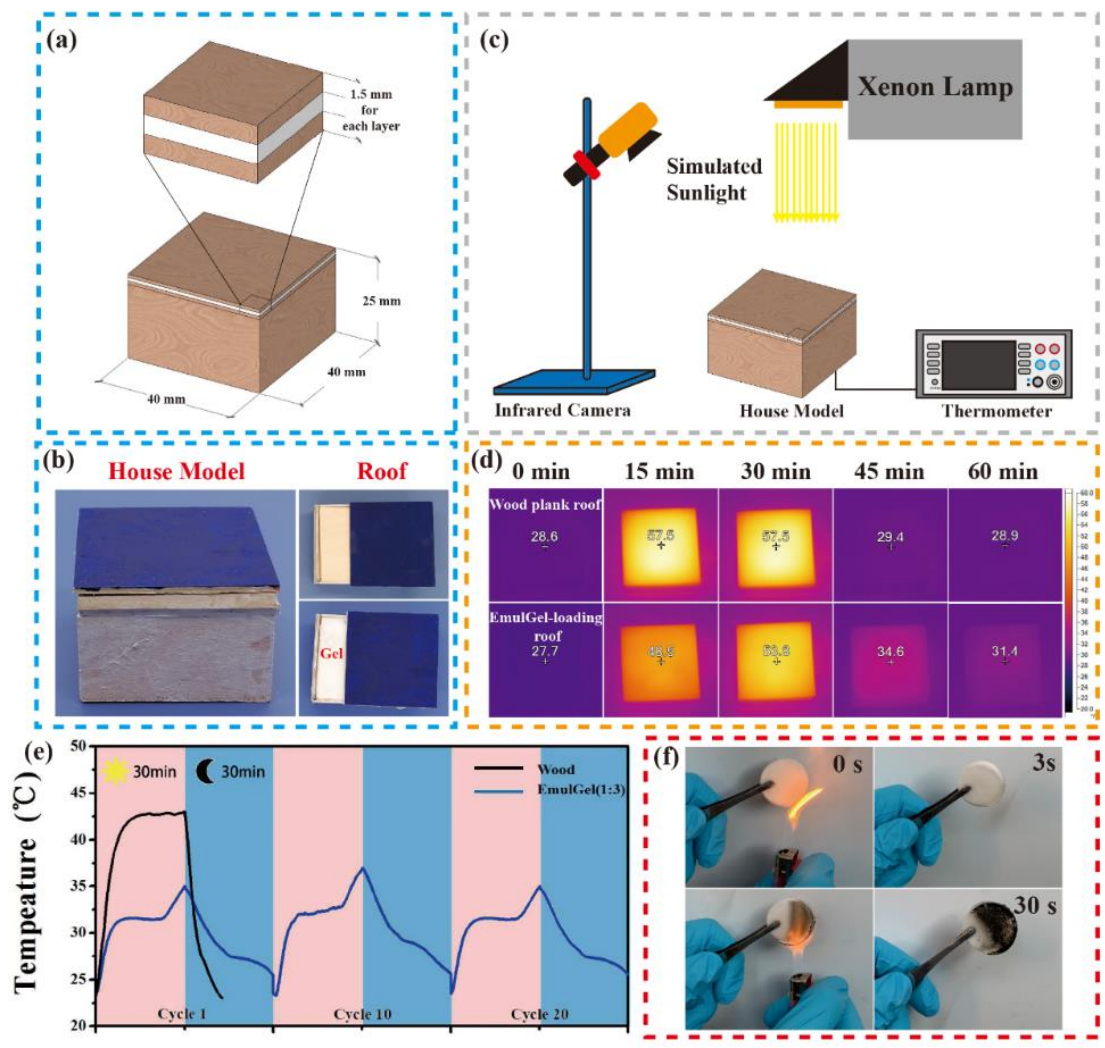

Figure 7.5 Passive energy management with EmulGel-loaded roof in a wooden house model: (a) schematic and (b) photographs of a lab-made wooden house model. (c) Schematic of the house day/night model testing arrangement used. (d) Infrared images of the roofs showing temperature variations during one day/night cycle. (e) The inner temperature of wooden house models for up to 20 cycles. (f) Photographs that demonstrate the non-flammable characteristic of EmulGel (1:3).

As is shown in Figure 7.5d, within one day/night cycle, the EmulGel-loaded roof reached a maximum temperature of $53.8^{\circ} \mathrm{C}$, which was $3.7^{\circ} \mathrm{C}$ lower than the control. What is even more striking is the observation that the plain wooden roof was heated to its maximum temperature of $57.5^{\circ} \mathrm{C}$ in $15 \mathrm{~min}$, by which time the EmulGel roof reached a temperature of only $48.5^{\circ} \mathrm{C}$, thus a $9{ }^{\circ} \mathrm{C}$ lower value. Clearly, both heating and cooling were delayed with the EmulGel-loaded roof, which was still above room temperature $30 \mathrm{~min}$ after irradiation stopped, while the plain wood roof cooled back to room temperature within $15 \mathrm{~min}$. The temperature inside the model houses changed 
following similar trends (Figure 7.5e), with a temperature difference of about $7.5{ }^{\circ} \mathrm{C}$ recorded between the EmulGel-loaded house and the control. In fact, temperature rise inside the model house to above the transition temperature of the EmulGel $\left(\sim 36{ }^{\circ} \mathrm{C}\right)$ was delayed by about 25 min for the house with an EmulGel-loaded roof. By contrast, the inner temperature of the control house quickly reached its maximum temperature of $\sim 42{ }^{\circ} \mathrm{C}$ in $8 \mathrm{~min}$. Within the 20 test cycles, the thin layer of EmulGel (1.5 mm thick) loaded in the roof mitigated temperature variations during the on-and-off irradiation effectively.

We again emphasize that the material has the added feature of being highly nonflammable. Figure 7.5f showed that the EmulGel still failed to ignite after 30 seconds of flame exposure. Overall, these results indicate that EmulGels have an excellent potential in passive building thermal management applications.

\subsection{Conclusions}

In conclusion, we developed and studied a non-flammable and shape-stable PCM in the form of an emulsion gel constructed from eicosane and DHPD via Pickering emulsion templated dual phase polymerization. The mechanical properties of EmulGels were tunable by changing the ratio of DHPD to eicosane. The EmulGel can also be shaped and fixed into temporary forms. EmulGel (1:3) displayed no supercooling and had a latent heat of $213.2 \mathrm{~J} / \mathrm{g}$. After 500 thermal cycles the latent heat of EmulGel was still $98 \%$ of its original value. Results of wooden house model day/night simulation tests and the flammability tests collectively suggest that EmulGel compares favorably to existing PCMs to be used in passive building thermal management systems.

\subsection{Experimental sections}

\subsubsection{Materials}

Dodecyl methacrylate (DMA), 2,2-Azobis (2-methylpropionitrile) (AIBN), and ethylene glycol dimethacrylate (EGDMA) were purchased from Shanghai Titan Scientific Co., Ltd. Eicosane was purchased from Acros. Fumed Silica $\left(\mathrm{F}-\mathrm{SiO}_{2}\right)$ was purchased from NIPPON AEROSIL Co., Ltd. Methyl methacrylate (MMA), Span80, DHPD, sodium hydroxide ( $\mathrm{NaOH}$ ), acrylic acid (AA), N.N'-methylenebisacrylamide (MBA), and potassium peroxydisulfate (KPS) were acquired from Sinopharm Chemical Reagent CO., Ltd. All chemicals were used as received. 


\subsubsection{Methods}

\section{Preparation of the emulsion gels}

Eicosane $(2.000 \mathrm{~g})$ was heated to melt in a $50{ }^{\circ} \mathrm{C}$ water bath. MMA $(0.300 \mathrm{~g})$, DMA (0.700 g), AIBN (0.020 g) and EGDMA (0.020 g) were added into the molten eicosane and stirred until fully dissolved. The obtained solution was used as the oily phase. Span80 (0.120 g) and $\mathrm{F}_{-} \mathrm{SiO}_{2}(0.120 \mathrm{~g})$ were added into the oil phase as emulsifiers and the mixture was shaken by a vortex mixer to obtain a uniform dispersion.

DHPD (6-9 g) was heated to melt in a $50{ }^{\circ} \mathrm{C}$ water bath, to which $\mathrm{NaOH}(0.167 \mathrm{~g})$ was added and shaken until complete dissolution. AA $(0.300 \mathrm{~g})$ was then added to the solution dropwise to neutralize excess $\mathrm{NaOH}$ to get sodium acrylate (NaAA), followed by sequential addition of MBA $(0.003 \mathrm{~g})$ and KPS $(0.003 \mathrm{~g})$. This mixture was used as the aqueous phase.

The oily phase and aqueous phase were combined at $50{ }^{\circ} \mathrm{C}$, respectively, and a water-in-oil (w/o) emulsion was obtained by subjecting the mixture to homogenization at $12000 \mathrm{rpm}$ for $2 \mathrm{~min}$. The emulsion was placed in an oven set to $70{ }^{\circ} \mathrm{C}$ for $3 \mathrm{~h}$ to allow polymerization of the acrylate monomers to form the emulsion hybrid gel (EmulGel). EmulGels with different mass ratio of eicosane to DHPD (1:2.5, 1:3, 1:3.5, 1:4, 1:4.5) were prepared and denoted as EmulGel (1:2.5), EmulGel (1:3), EmulGel (1:3.5,) EmulGel (1:4), and EmulGel (1:4.5), respectively.

\section{Preparation of the hydrogels}

DHPD $(6.000 \mathrm{~g})$ was heated to melt in a $50{ }^{\circ} \mathrm{C}$ water bath, to which $\mathrm{NaOH}(0.167$ g) was added and shaken until complete dissolution. AA (0.300 g) was then added to the solution dropwise to neutralize the excess $\mathrm{NaOH}$ to get $\mathrm{NaAA}$, followed by addition of MBA $(0.003 \mathrm{~g})$ and KPS $(0.003 \mathrm{~g})$. The mixture was placed in an oven set at $70{ }^{\circ} \mathrm{C}$ for $1 \mathrm{~h}$ to obtain the hydrogels denoted as DHPD-Gel.

\section{Preparation of the oily gels}

Eicosane $(2.000 \mathrm{~g})$ was heated to melt in a $50{ }^{\circ} \mathrm{C}$ water bath. MMA $(0.300 \mathrm{~g})$, $\operatorname{DMA}(0.700 \mathrm{~g}), \operatorname{AIBN}(0.020 \mathrm{~g})$ and $\operatorname{EGDMA}(0.020 \mathrm{~g})$ were added into the molten eicosane and stirred until fully dissolved. The obtained solution was put in an oven set at $70{ }^{\circ} \mathrm{C}$ for $3 \mathrm{~h}$ to obtain the oil gel denoted as Eicosane-Gel. 


\subsubsection{Characterization}

\section{Optical microscopy}

The optical photographs of the w/o emulsions were captured using an Eclipse microscope equipped with a digital camera (Nikon 80i, Japan) at above the melting temperatures of DHPD and eicosane. The mean droplet diameter values of w/o emulsions were obtained using the Nanomeasurer Software. At least 350 droplets were taken to calculate the mean droplet diameter for each DHPD : Eicosane ratio.

\section{Scanning electron microscopy}

A scanning electron microscope (SEM, TM3030, Hitachi, Japan) was used to observe the fracture surfaces of the EmulGels. Prior to testing, the EmulGels were fractured after frozen in liquid nitrogen, and then spray-coated with a layer of gold under vacuum.

\section{Mechanical properties}

The compressive stress-strain properties of the EmulGels were measured using a universal testing machine (HY 940F, China) at a compression speed of $50 \mathrm{~mm} / \mathrm{min}$. The maximum strain applied was $80 \%$. The samples (4-6 mm thick) were cut by a puncher into circular sheets of $8 \mathrm{~mm}$ diameter.

\section{Shape memory}

To show that the EmulGel has shape memory properties, An EmulGel (1:3) strip was heated to $60{ }^{\circ} \mathrm{C}$ and reshaped by subjecting to an external stress. The reshaped gel was allowed to cool to ambient temperature. Finally, the stress was removed and the EmulGel was heated back to $60{ }^{\circ} \mathrm{C}$ to allow recovery to the original shape.

\section{Leakage-proof properties}

The gel samples (EmulGel (1:3) and a control without polymerization) were placed on filter papers and placed in an oven set at $60^{\circ} \mathrm{C}$ for 30 mins.

\section{Differential Scanning Calorimetry}

The thermal properties of DHPD, eicosane and EmulGel were analyzed using differential scanning calorimetry (DSC, 214Polyma, NETZSCH, Germany). Under nitrogen purge, the samples were heated from $-10{ }^{\circ} \mathrm{C}$ to $70{ }^{\circ} \mathrm{C}$ and then cooled to $10{ }^{\circ} \mathrm{C}$ at a constant rate of $10^{\circ} \mathrm{C} / \mathrm{min}$. 
Thermal cycling tests

The cycling tests of DHPD and EmulGel were performed using a custom-made instrument, which is shown in Figure 7-S6. The samples were placed in a heating plate for $2 \mathrm{~min}$ and then cooled to and maintained at room temperature for $5 \mathrm{~min}$. The procedure was repeated for 500 times.

Thermogravimetric analysis

The thermal properties of DHPD, DHPD-Gel, eicosane, Eicosane-Gel and EmulGel (1:3) were evaluated by thermogravimetric analysis (TGA, 209F3, Netzsch, Germany). Under nitrogen atmosphere $(20 \mathrm{~mL} / \mathrm{min}$ gas flow $)$, the samples were heated from 20 to $600{ }^{\circ} \mathrm{C}$ at a rate of $10{ }^{\circ} \mathrm{C} / \mathrm{min}$.

\section{Encapsulation ratio}

The encapsulation ratio of EmulGel was calculated using the following equation ${ }^{49}$.

$$
E_{n}=\left(\frac{\Delta H_{C 1(\text { EmulGel })}}{\Delta H_{C 1(\text { DHPD })}}+\frac{\Delta H_{C 2(\text { EmulGel })}}{\Delta H_{C 2 \text { (Eicosane })}}\right) \times 100 \%
$$

where $E_{n}$ is the encapsulation ratio and $\Delta H_{c}$ is the enthalpy of crystallization of the respective phases.

\section{Measurement of water loss in open environments}

DHPD, DHPD-Gel, and EmulGel (1:3) (respectively 3 g) were weighed before and after being heated in an oven set at $60^{\circ} \mathrm{C}$. The difference in mass of each sample was recorded every 10 mins.

\section{Degree of supercooling}

The degree of supercooling of the samples was determined based on their cooling curves in thermal analysis. The cooling curves of all the samples were obtained by heating and cooling the samples in water baths set at $60{ }^{\circ} \mathrm{C}$ and $0{ }^{\circ} \mathrm{C}$ respectively, and record the temperature of the samples every $5 \mathrm{~s}$ using a DMM650 multimeter.

\section{Evaluating EmulGel in model building thermal management systems}

Two wooden model houses (basswood) sized $40 \mathrm{~mm} \times 40 \mathrm{~mm} \times 25 \mathrm{~mm}(\mathrm{~L} \times \mathrm{W}$ $\times$ H) were built, with the roof of one constructed by sandwiching a $40 \mathrm{~mm} \times 40 \mathrm{~mm}$ $\times 1.5 \mathrm{~mm}$ EmulGel (1:3) between two wood planks. The total thickness of the roofs was kept at $4.5 \mathrm{~mm}$. A xenon lamp (PLS-SXE300+) was used to simulate sunlight. An infrared camera (IR camera, Fluke TiX450, USA) was used to monitor temperature 
variations of roofs at working voltages ranged from 2 to $6 \mathrm{~V}$. Infrared pictures of each roof were taken every $15 \mathrm{~min}$.

Inner temperature values of the wooden model houses were recorded using a DMM650 multimeter for each heating and cooling cycle. The houses were irradiated for $30 \mathrm{~min}$ and then kept in the dark for $30 \mathrm{~min}$. This procedure was repeated for 20 cycles. 


\section{Appendix}
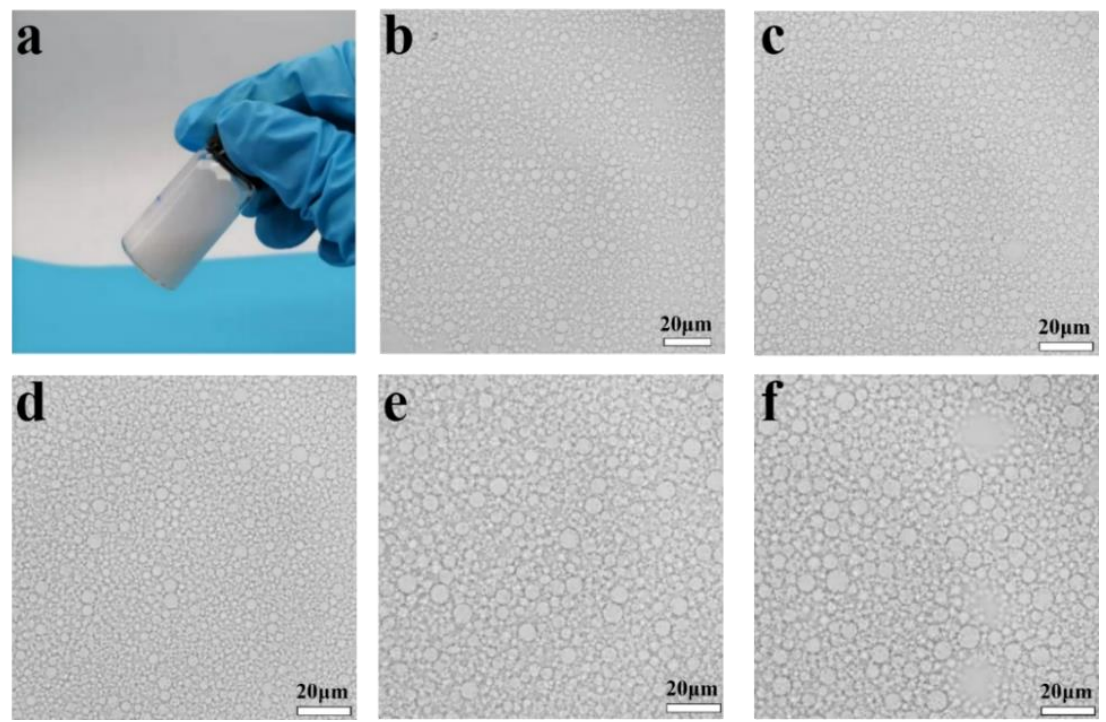

Figure 7-S1 (a) Optical photographs of w/o emulison and opitical microscopy images of emulsions with different eicosane to DHPD ratios ((b) 1:2.5, (c) 1:3, (d) 1:3.5, (e) 1:4, (f) 1:4.5).
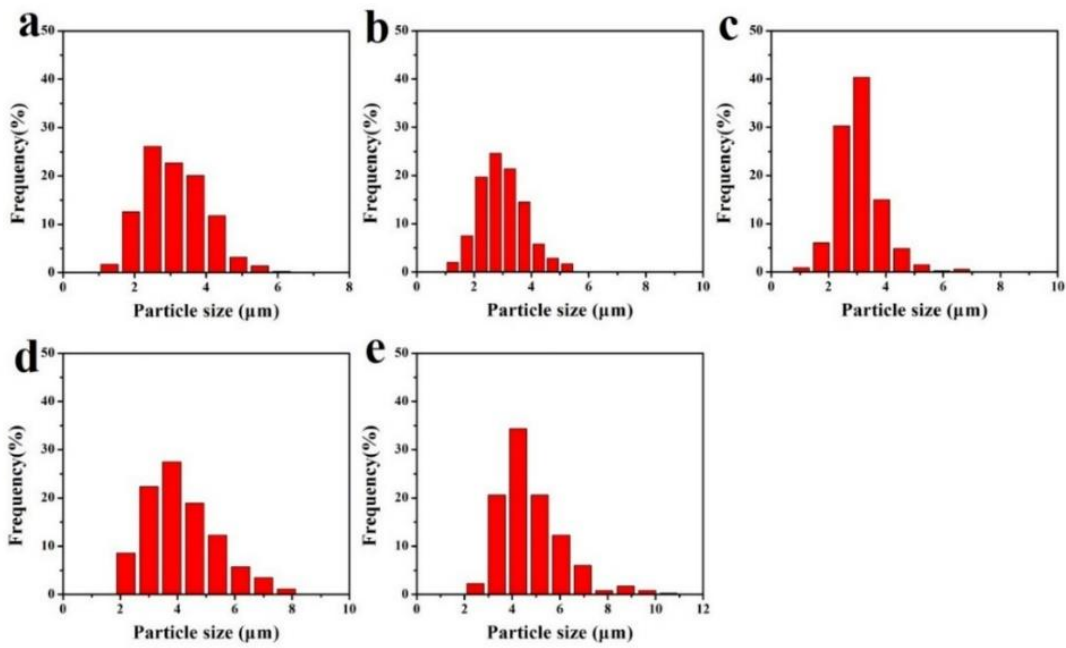

Figure 7-S2 Particle size of emulsions with different eicosane to DHPD ratios ((a) 1:2.5, (b) $1: 3$, (c) $1: 3.5$, (d) $1: 4$, (e) $1: 4.5$ ). 

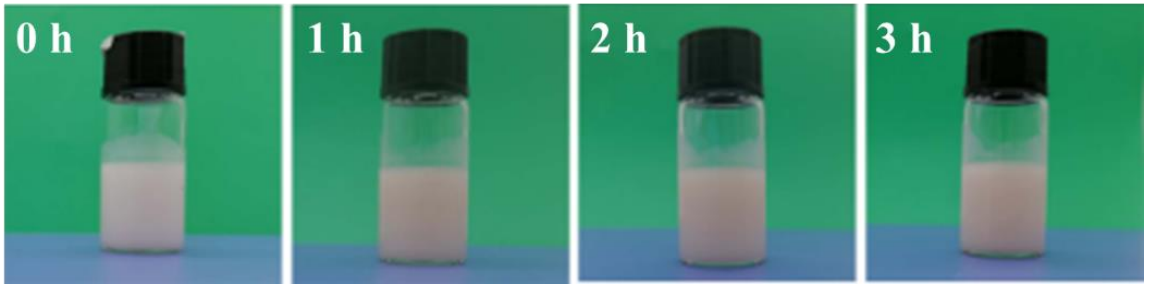

Figure 7-S3 Photographs of emulsions exposed to $70{ }^{\circ} \mathrm{C}$ for $3 \mathrm{~h}$.

Table 7-S1 DSC analysis of DHPD, eicosane and EmulGel (1:3).

\begin{tabular}{ccccc}
\hline Sample & $\Delta \mathbf{H}_{\mathbf{m}}(\mathbf{J} / \mathbf{g})$ & $\Delta \mathbf{H}_{\mathbf{c 1}}(\mathbf{J} / \mathbf{g})$ & $\Delta \mathbf{H}_{\mathbf{c 2}}(\mathbf{J} / \mathbf{g})$ & $\mathbf{E}_{\mathbf{n}}(\mathbf{\%})$ \\
\hline DHPD & 278 & 229.9 & - & - \\
Eicosane & 257.7 & - & 254.5 & - \\
EmulGel (1:3) & 213.2 & 129.3 & 55.8 & 78.1 \\
\hline
\end{tabular}
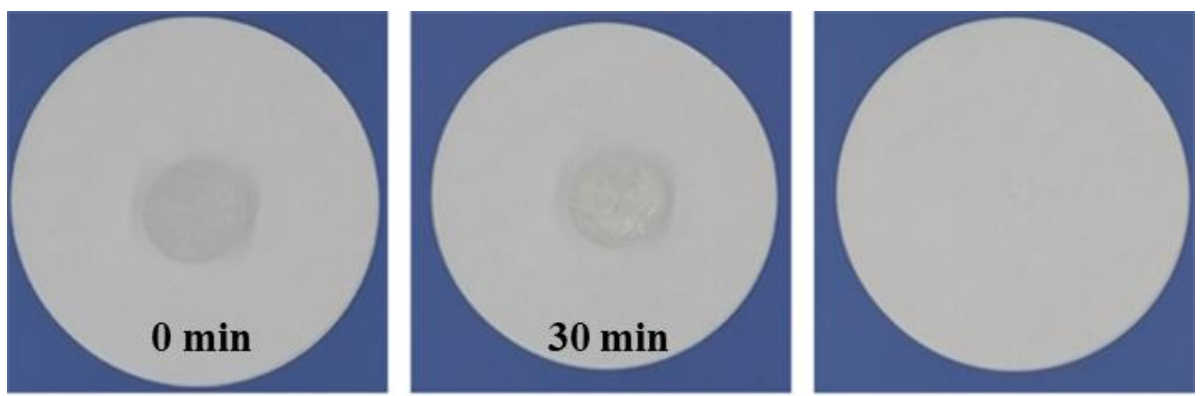

Figure 7-S4 Leak-proof characteristic testing of Eicosane-Gel. 
a

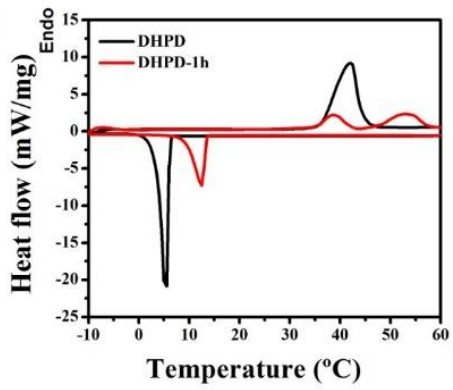

C

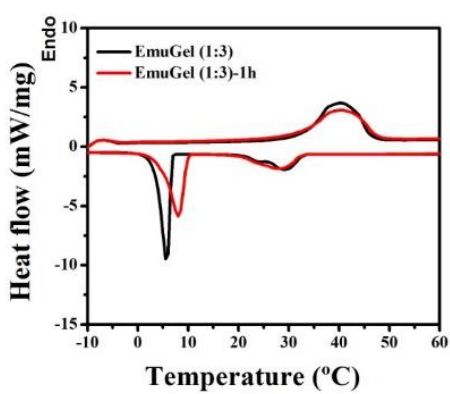

b

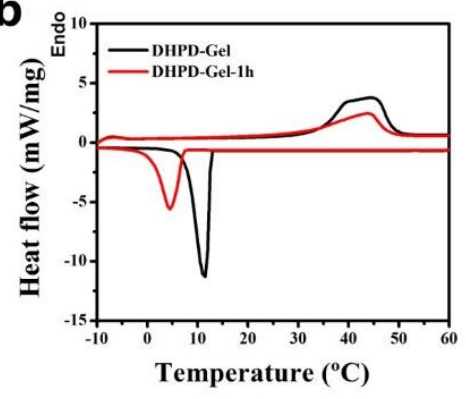

Figure 7-S5 DSC traces of DHPD, DHPD-Gel and EmulGel (1:3) after water loss tests.

Table 7-S2 DSC data of DHPD, DHPD-Gel and EmulGel (1:3) after water loss test.

\begin{tabular}{llll}
\hline Sample & $\Delta \mathbf{H}_{\mathbf{m}}(\mathbf{J} / \mathbf{g})$ & $\Delta \mathbf{H}_{\mathbf{c}}(\mathbf{J} / \mathbf{g})$ & Ratio (\%) \\
\hline DHPD & 278.0 & 229.9 & 100 \\
DHPD-1h & 134.9 & 107.7 & 48.5 \\
DHPD-Gel & 222.7 & 203.8 & 100 \\
DHPD-Gel-1h & 149.3 & 134.7 & 67.0 \\
EmulGel (1:3) & 191.6 & 190.5 & 100 \\
EmulGel (1:3)-1h & 183.3 & 180.7 & 95.7 \\
\hline
\end{tabular}




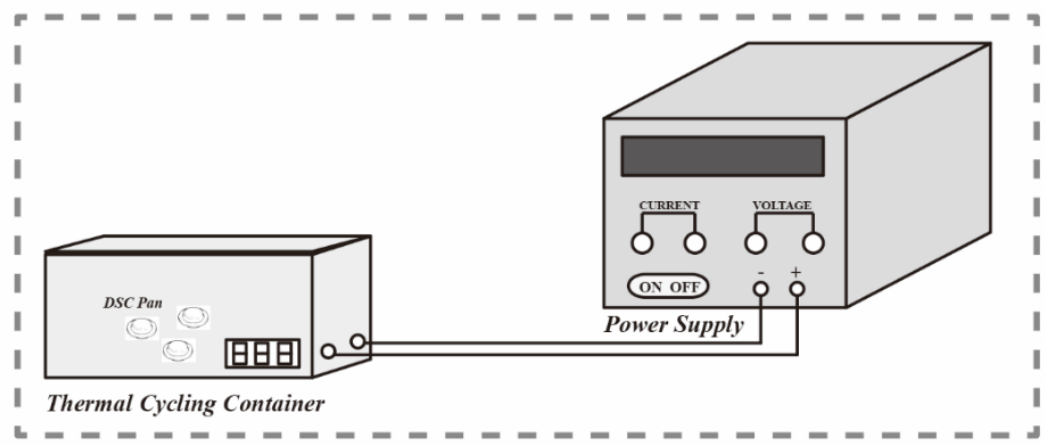

Figure 7-S6 Schematic diagram of the custom-made thermal cycling instrument. 


\section{References}

[1] Rotzetter, A. C.; Schumacher, C. M.; Bubenhofer, S. B.; Grass, R. N.; Gerber, L. C.; Zeltner, M.; Stark, W. J. Thermoresponsive polymer induced sweating surfaces as an efficient way to passively cool buildings. Advanced Materials 2012, 24 (39), 5352-5356.

[2] Wang, J.; Zhai, Z. J.; Jing, Y.; Zhang, C. Particle swarm optimization for redundant building cooling heating and power system. Applied Energy 2010, 87 (12), 3668-3679.

[3] Chwieduk, D. Towards sustainable-energy buildings. Applied Energy 2003, 76 (1-3), 211-217.

[4] Raji, B.; Tenpierik, M. J.; van den Dobbelsteen, A. The impact of greening systems on building energy performance: A literature review. Renewable and Sustainable Energy Reviews 2015, 45, 610-623.

[5] Waqas, A.; Din, Z. U. Phase change material (PCM) storage for free cooling of buildings-A review. Renewable and Sustainable Energy Reviews 2013, 18, 607625 .

[6] Liu, S.; Duvigneau, J.; Vancso, G. J. Nanocellular polymer foams as promising high performance thermal insulation materials. European Polymer Journal 2015, $65,33-45$.

[7] Mohamed, S. A.; Al-Sulaiman, F. A.; Ibrahim, N. I.; Zahir, M. H.; Al-Ahmed, A.; Saidur, R.; Yılbaş, B.; Sahin, A. A review on current status and challenges of inorganic phase change materials for thermal energy storage systems. Renewable and Sustainable Energy Reviews 2017, 70, 1072-1089.

[8] Milian, Y. E.; Gutierrez, A.; Grageda, M.; Ushak, S. A review on encapsulation techniques for inorganic phase change materials and the influence on their thermophysical properties. Renewable and Sustainable Energy Reviews 2017, 73, 983-999.

[9] Wuttig, M.; Yamada, N. Phase-change materials for rewriteable data storage. Nature Materials 2007, 6 (11), 824-832.

[10] Yang, J.; Tang, L.-S.; Bai, L.; Bao, R.-Y.; Liu, Z.-Y.; Xie, B.-H.; Yang, M.-B.; Yang, W. High-performance composite phase change materials for energy conversion based on macroscopically three-dimensional structural materials. Materials Horizons 2019, 6 (2), 250-273. 
[11] Liu, L.; Fan, X.; Zhang, Y.; Zhang, S.; Wang, W.; Jin, X.; Tang, B. Novel biobased phase change materials with high enthalpy for thermal energy storage. Applied Energy 2020, 268, 114979.

[12] Chen, X.; Gao, H.; Hai, G.; Jia, D.; Xing, L.; Chen, S.; Cheng, P.; Han, M.; Dong, W.; Wang, G. Carbon nanotube bundles assembled flexible hierarchical framework based phase change material composites for thermal energy harvesting and thermotherapy. Energy Storage Materials 2020, 26, 129-137.

[13] Nazir, H.; Batool, M.; Osorio, F. J. B.; Isaza-Ruiz, M.; Xu, X.; Vignarooban, K.; Phelan, P.; Kannan, A. M. Recent developments in phase change materials for energy storage applications: a review. International Journal of Heat and Mass Transfer 2019, 129, 491-523.

[14] Su, W.; Darkwa, J.; Kokogiannakis, G. Review of solid-liquid phase change materials and their encapsulation technologies. Renewable and Sustainable Energy Reviews 2015, 48, 373-391.

[15] Cabeza, L. F.; Castell, A.; Barreneche, C. d.; De Gracia, A.; Fernández, A. Materials used as PCM in thermal energy storage in buildings: A review. Renewable and Sustainable Energy Reviews 2011, 15 (3), 1675-1695.

[16] Yang, J.; Qi, G.-Q.; Bao, R.-Y.; Yi, K.; Li, M.; Peng, L.; Cai, Z.; Yang, M.-B.; Wei, D.; Yang, W. Hybridizing graphene aerogel into three-dimensional graphene foam for high-performance composite phase change materials. Energy Storage Materials 2018, 13, 88-95.

[17] Royon, L.; Karim, L.; Bontemps, A. Thermal energy storage and release of a new component with PCM for integration in floors for thermal management of buildings. Energy and Buildings 2013, 63, 29-35.

[18] Wang, Y.; Yu, K.; Ling, X. Experimental and modeling study on thermal performance of hydrated salt latent heat thermal energy storage system. Energy Conversion and Management 2019, 198, 111796.

[19] Zhang, H.; Baeyens, J.; Caceres, G.; Degreve, J.; Lv, Y. Thermal energy storage: Recent developments and practical aspects. Progress in Energy and Combustion Science 2016, 53, 1-40.

[20] Deng, Y.; Li, J.; Deng, Y.; Nian, H.; Jiang, H. Supercooling suppression and thermal conductivity enhancement of $\mathrm{Na}_{2} \mathrm{HPO}_{4} \cdot 12 \mathrm{H}_{2} \mathrm{O} /$ expanded vermiculite form-stable composite phase change materials with alumina for heat storage. 
ACS Sustainable Chemistry \& Engineering 2018, 6 (5), 6792-6801.

[21] Wong-Pinto, L.-S.; Milian, Y.; Ushak, S. Progress on use of nanoparticles in salt hydrates as phase change materials. Renewable and Sustainable Energy Reviews 2020, 122, 109727.

[22] Xie, N.; Luo, J.; Li, Z.; Huang, Z.; Gao, X.; Fang, Y.; Zhang, Z. Salt hydrate/expanded vermiculite composite as a form-stable phase change material for building energy storage. Solar Energy Materials and Solar Cells 2019, 189, 33-42.

[23] Graham, M.; Shchukina, E.; De Castro, P. F.; Shchukin, D. Nanocapsules containing salt hydrate phase change materials for thermal energy storage. Journal of Materials Chemistry A 2016, 4 (43), 16906-16912.

[24] Graham, M.; Coca-Clemente, J. A.; Shchukina, E.; Shchukin, D. Nanoencapsulated crystallohydrate mixtures for advanced thermal energy storage. Journal of Materials Chemistry A 2017, 5 (26), 13683-13691.

[25] Zhang, Z.; Lian, Y.; Xu, X.; Xu, X.; Fang, G.; Gu, M. Synthesis and characterization of microencapsulated sodium sulfate decahydrate as phase change energy storage materials. Applied Energy 2019, 255, 113830.

[26] Liu, Y.; Yang, Y.; Li, S. Graphene oxide modified hydrate salt hydrogels: Formstable phase change materials for smart thermal management. Journal of Materials Chemistry A 2016, 4 (46), 18134-18143.

[27] Graham, M.; Smith, J.; Bilton, M.; Shchukina, E.; Novikov, A. A.; Vinokurov, V.; Shchukin, D. G. Highly Stable Energy Capsules with Nano-SiO 2 Pickering Shell for Thermal Energy Storage and Release. ACS Nano 2020, 14 (7), 8894-8901.

[28] Gawron, K.; Schröder, J. Properties of some salt hydrates for latent heat storage. International Journal of Energy Research 1977, 1 (4), 351-363.

[29] Ushak, S.; Gutierrez, A.; Barreneche, C.; Fernandez, A. I.; Grágeda, M.; Cabeza, L. F. Reduction of the subcooling of bischofite with the use of nucleatings agents. Solar Energy Materials and Solar Cells 2016, 157, 1011-1018.

[30] Pilar, R.; Svoboda, L.; Honcova, P.; Oravova, L. Study of magnesium chloride hexahydrate as heat storage material. Thermochimica Acta 2012, 546, 81-86.

[31] Lane, G. A. Phase change materials for energy storage nucleation to prevent supercooling. Solar Energy Materials and Solar Cells 1992, 27 (2), 135-160.

[32] Li, X.; Zhou, Y.; Nian, H.; Zhu, F.; Ren, X.; Dong, O.; Hai, C.; Shen, Y.; Zeng, 
J. Preparation and thermal energy storage studies of $\mathrm{CH}_{3} \mathrm{COONa} \cdot 3 \mathrm{H}_{2} \mathrm{O}-\mathrm{KCl}$ composites salt system with enhanced phase change performance. Applied Thermal Engineering 2016, 102, 708-715.

[33] Zhang, X.; Li, X.; Zhou, Y.; Hai, C.; Shen, Y.; Ren, X.; Zeng, J. Calcium chloride hexahydrate/diatomite/paraffin as composite shape-stabilized phase-change material for thermal energy storage. Energy \& Fuels 2018, 32 (1), 916-921.

[34] Shen, C.; Li, X.; Yang, G.; Wang, Y.; Zhao, L.; Mao, Z.; Wang, B.; Feng, X.; Sui, $\mathrm{X}$. Shape-stabilized hydrated salt/paraffin composite phase change materials for advanced thermal energy storage and management. Chemical Engineering Journal 2020, 385, 123958.

[35] Hohenstein, W.; Mark, H. Polymerization of olefins and diolefins in suspension and emulsion. Part I. Journal of Polymer Science 1946, 1 (2), 127-145.

[36] Chen, T.; Colver, P. J.; Bon, S. A. Organic-inorganic hybrid hollow spheres prepared from $\mathrm{TiO}_{2}$-stabilized Pickering emulsion polymerization. Advanced Materials 2007, 19 (17), 2286-2289.

[37] Binks, B.; Lumsdon, S. Catastrophic phase inversion of water-in-oil emulsions stabilized by hydrophobic silica. Langmuir 2000, 16 (6), 2539-2547.

[38] Cunningham, V. J.; Giakoumatos, E. C.; Ireland, P. M.; Mable, C. J.; Armes, S. P.; Wanless, E. J. Giant Pickering droplets: effect of nanoparticle size and morphology on stability. Langmuir 2017, 33 (31), 7669-7679.

[39] Xiao, Q.; Fan, J.; Li, L.; Xu, T.; Yuan, W. Solar thermal energy storage based on sodium acetate trihydrate phase change hydrogels with excellent light-to-thermal conversion performance. Energy 2018, 165, 1240-1247.

[40] Wang, T.; Wu, N.; Li, H.; Lu, Q. L.; Jiang, Y. Preparation and properties of a form-stable phase-change hydrogel for thermal energy storage. Journal of Applied Polymer Science 2016, 133 (34).

[41] Liu, Y.; Yu, K.; Gao, X.; Ren, M.; Jia, M.; Yang, Y. Enhanced thermal properties of hydrate salt/poly (acrylate sodium) copolymer hydrogel as form-stable phase change material via incorporation of hydroxyl carbon nanotubes. Solar Energy Materials and Solar Cells 2020, 208, 110387.

[42] Gui, H.; Zhang, T.; Guo, Q. Closed-cell, emulsion-templated hydrogels for latent heat storage applications. Polymer Chemistry 2018, 9 (29), 3970-3973.

[43]Deng, Y.; Yang, L. Preparation and characterization of polyethylene glycol (PEG) 
hydrogel as shape-stabilized phase change material. Applied Thermal Engineering 2017, 114, 1014-1017.

[44] Shen, C.; Li, X.; Yang, G.; Wang, Y.; Zhao, L.; Mao, Z.; Wang, B.; Feng, X.; Sui, $X$. Shape-stabilized hydrated salt/paraffin composite phase change materials for advanced thermal energy storage and management. Chemical Engineering Journal 2020, 385, 123958.

[45] Zhou, L.; Sun, Z.; Lu, L.; Li, J.; Li, C.; Huang, H. Effect of organic phase change material and surfactant on $\mathrm{HCFC} 141 \mathrm{~b}$ hydrate nucleation in quiescent conditions. Chemical Engineering Science 2020, 228, 115976.

[46] Tai, Z.; Wei, J.; Zhou, J.; Liao, Y.; Wu, C.; Shang, Y.; Wang, B.; Wang, Q. Watermediated crystallohydrate-polymer composite as a phase-change electrolyte. Nature Communication 2020, 11 (1), 1843, 1-10.

[47] Zhao, Z.; Zhang, K.; Liu, Y.; Zhou, J.; Liu, M. Highly stretchable, shape memory organohydrogels using phase-transition microinclusions. Advanced Materials 2017, 29 (33), 1701695.

[48] Zhao, Z.; Li, C.; Dong, Z.; Yang, Y.; Zhang, L.; Zhuo, S.; Zhou, X.; Xu, Y.; Jiang, L.; Liu, M. Adaptive superamphiphilic organohydrogels with reconfigurable surface topography for programming unidirectional liquid transport. Advanced Functional Materials 2019, 29 (16), 1807858.

[49] Lashgari, S.; Arabi, H.; Mahdavian, A. R.; Ambrogi, V. Thermal and morphological studies on novel PCM microcapsules containing n-hexadecane as the core in a flexible shell. Applied Energy 2017, 190, 612-622. 
Outlook: Possible Future Trends in Designer Particles as Pickering Emulsifiers 
As presented and discussed in this Thesis, Pickering emulsion templates provide a promising way to direct the morphology and function of polymer-based hybrid materials. Nanocellulose was used as one of the emulsifying agents to obtain engineered materials. We demonstrated that it can be well dispersed in the PLA matrix using Pickering emulsion templating. Dyes were also dispersed in a PMMA matrix using dyed-nanocellulose as Pickering emulsion templates to prepare colored composites. Two different hydrophilic and hydrophobic properties of phase change materials have been combined effectively and stably using the Pickering emulsion approach. In addition these successful applications, there is a significant potential to further broaden and enhance the applicability of Pickering emulsion templating method. For instance, one can focus on improving the compatibility between CNC and PLA to boost the reinforcing effect of CNCs in PLA. As another example, we mention that the performance of our phase change gel platforms could be further enhanced by increasing their thermal conductivity, which could be tackled by the Pickering approach.

The Pickering particles play an essential role in the templating process, i.e. they act as emulsifiers and also as function contributors ${ }^{1-3}$. The dimensions and properties of Pickering particles determine the overall properties of the resulting hybrid materials, for instance, the mechanical or optical properties. In this Outlook, we will highlight and discuss some exciting possible future directions, including particle functionalization (8.1) and the use of multiple particle types in hybrid systems (8.2)

\subsection{Particle functionalization}

When using Pickering emulsion templating, cellulose nanocrystals were readily dispersed in various matrixes without surface modification. Such modifications are often required for other mixing methods, e.g. in melt blending. A good coupling between matrix and particle in the formed hybrids remains essential when considering, for instance, mechanical reinforcement. In Chapter 3, nanocellulose was well dispersed in a PLA matrix using Pickering emulsion templating, but the poor compatibility of PLA and nanocellulose needs to be enhanced for further property improvements. Therefore, we considered surface modifications of the stabilizing particles. For instance, silane coupling agents with adequate substituents can improve the compatibility of PLA and nanocellulose in Pickering emulsion templated systems. Substituted silane coupling agents with side groups such as amine can have good compatibility with $\mathrm{PLA}^{4}$ and can easily react with nanocellulose. Furthermore, silane- 
modified nanocellulose, when properly selected, has a good hydrophilic balance to stabilize a Pickering emulsion. For example, Tang and coworkers used 3aminopropyltriethoxysilane (KH-550) to modify nanocellulose to improve the compatibility of PLA and nanocellulose ${ }^{5}$. They showed that the tensile strength and elongation at break of the composites were improved by $54 \%$ and $61 \%$, respectively, using only 0.5 wt. $\%$ modified-nanocellulose.

Polyethylene glycol (PEG) can also improve the compatibility between PLA and nanocellulose ${ }^{6}$. PEG can be readily combined with nanocellulose by physical adsorption ${ }^{7}$. A schematic of hybrid CNC-PEG particles is shown in Figure 8.1. The hybrid particles are still good emulsifiers for the Pickering method due to the good emulsifying properties of the PEG chains. These, and similar, particle surface modification strategies are expected to improve the compatibility between CNC particles and PLA such that an increased reinforcement for the hybrid material can be achieved.

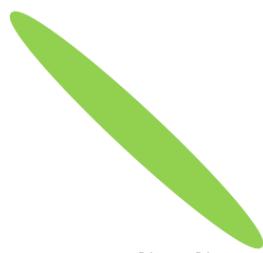

CNC

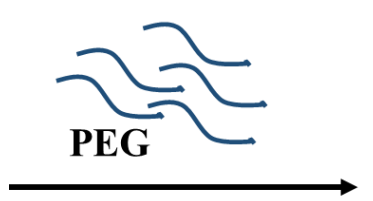

Physical adsorption

Figure 8.1 Schematic of preparation of hybrid of CNC-PEG systems.

In Chapter 6 we presented that the addition of nanocellulose crystals by Pickering emulsion templating, followed by melt blending, can yield PLA foams with a decreased cell size and increased cell density. While the addition of a heterogeneous phase often results in enhanced foam cell nucleation, it requires particle surface design and engineering to exploit the potential nucleating capabilities of these particles. For instance, our group has reported that the addition of nanoparticles with $\mathrm{CO}_{2}$-philic functionalities ${ }^{8}$, controlled interfacial interactions ${ }^{9}$, and increased surface roughness ${ }^{10}$ significantly enhances the nucleating efficiency of silica-based particles in thermoplastic foams. The main reason that this strategy is effective for these particles is that they increase the $\mathrm{CO}_{2}$ concentration in close proximity of the surface of nucleating particles, which is favorable for foam cell nucleation. This is schematically 
shown in Figure 8.2 for a modified cellulose nanocrystal.

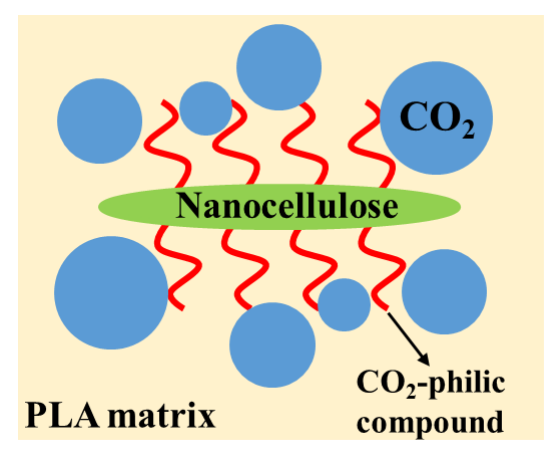

Figure 8.2 Schematic of increased $\mathrm{CO}_{2}$ absorption near the surface of a modified nanocellulose with a $\mathrm{CO}_{2}$-philic compound.

Silane coupling agents containing amino groups are often used as $\mathrm{CO}_{2}$-philic compounds in $\mathrm{CO}_{2}$ capture applications ${ }^{11}$. Therefore, these could be promising candidates to enhance the foam nucleation efficiency of cellulose nanocrystals. In preliminary experiments, we have used N-(2-aminoethyl)-3-(trimethoxysilyl) propylamine (AEAPTMS), and (3-aminopropyl) triethoxysilane (APTES) to modify nanocellulose for foam nucleation applications. However, the amino-modified nanocellulose particles did not yield PLA/CNC hybrid foams with improved foam morphologies. At this point, we assume that either not enough silane coupling agents reacted with the nanocellulose or that the silane coupling agents we used do not induce an increased $\mathrm{CO}_{2}$ absorption near the PLA/CNC interface. This must be investigated in future research.

Besides silane coupling agents, $\mathrm{CO}_{2}$-philic polymer chains have attracted much attention in foaming due to their high $\mathrm{CO}_{2}$ absorption capacity ${ }^{12}$. For nanocellulose modification, it is suggested to modify amino silane-modified CNC further with polydimethylsiloxane (PDMS) to enhance the nucleation efficiency of CNCs. This particle design is believed to be very promising since previous reports by our group have shown that PDMS grafted to spherical particles had a significant influence on the foaming behavior of $\mathrm{PMMA}^{8,13}$. Therefore, we highly recommend focusing on the development of CNC-PDMS core-shell particles in future research.

\subsection{Combination of different particle types}

Besides functionalization, using two or more different particle types is believed to 
open another window for designing functional polymer-based hybrid materials based on Pickering emulsion templating. Therefore, in this part, we will present some examples in which different particle types are combined. These can be a combination of two different Pickering particles or Pickering particles with non-Pickering particles.

For PLA/CNC composite foaming, another exciting direction is to study the influence of the texture of nanocellulose on PLA foaming behaviors. In a previous report by our group, it was shown that the surface texture of silica particles significantly increased the nucleation efficiency in PMMA foaming ${ }^{10}$. We would recommend investigating if, for instance, the silica deposition method ${ }^{14-15}$ can be employed to surface engineer the texture of CNC particles and utilize these particles as foam cell nucleating agents. Of particular relevance is to elucidate the role of a highly anisotropic particle on the foam cell nucleating behavior compared to the highly effective spherical nucleating particles extensively studied by our group.

In Chapter 7, by combing paraffin using Pickering emulsion templates, some characteristics, e.g. water-loss and degree of supercooling, of hydrated salts utilized in building thermal management were improved. But the low thermal conductivity of phase change gels remains a limitation for the widespread exploitation of the phase change gels. Utilization of thermally conductive Pickering particles has been reported as a successful strategy to improve the thermal conductivity of polymers ${ }^{16-17}$. For instance, Kim and coworkers ${ }^{18}$ reported that the thermal conductivity of polymer composites was synergistically improved by the simultaneous incorporation of graphene nanoplatelet and multi-walled carbon nanotube fillers into a polycarbonate matrix. To improve the thermal conductivity, we propose to use Pickering particles with high thermal conductivity in addition to, or to replace, fumed silica in the EmulGels (Chapter 7). Proposed particles to use are e.g. CNT, graphene oxide, carbon black, carbon fiber, and boron nitride.

The strategies for designing and engineering Pickering particles described in this Chapter provide many opportunities for developing novel, high-performance polymer-based hybrid materials based on Pickering emulsion templating as a key enabling, versatile and robust production platform. 


\section{References}

[1] Tang, M.; Wang, X.; Wu, F.; Liu, Y.; Zhang, S.; Pang, X.; Li, X.; Qiu, H. Au nanoparticle/graphene oxide hybrids as stabilizers for Pickering emulsions and Au nanoparticle/graphene oxide@ polystyrene microspheres. Carbon 2014, 71, $238-248$.

[2] Kim, Y. J.; Liu, Y. D.; Seo, Y.; Choi, H. J. Pickering-emulsion-polymerized polystyrene $/ \mathrm{Fe}_{2} \mathrm{O}_{3}$ composite particles and their magnetoresponsive characteristics. Langmuir 2013, 29 (16), 4959-4965.

[3] Chen, T.; Colver, P. J.; Bon, S. A. Organic-inorganic hybrid hollow spheres prepared from $\mathrm{TiO}_{2}$-stabilized Pickering emulsion polymerization. Advanced Materials 2007, 19 (17), 2286-2289.

[4] Qian, S.; Sheng, K. PLA toughened by bamboo cellulose nanowhiskers: Role of silane compatibilization on the PLA bionanocomposite properties. Composites Science and Technology 2017, 148, 59-69.

[5] Jin, K.; Tang, Y.; Zhu, X.; Zhou, Y. Polylactic acid based biocomposite films reinforced with silanized nanocrystalline cellulose. International Journal of Biological Macromolecules 2020, 162, 1109-1117.

[6] Oksman, K.; Mathew, A. P.; Bondeson, D.; Kvien, I. Manufacturing process of cellulose whiskers/polylactic acid nanocomposites. Composites Science and Technology 2006, 66 (15), 2776-2784.

[7] Cheng, D.; Wen, Y.; Wang, L.; An, X.; Zhu, X.; Ni, Y. Adsorption of polyethylene glycol (PEG) onto cellulose nano-crystals to improve its dispersity. Carbohydrate Polymers 2015, 123, 157-163.

[8] Liu, S.; Zoetebier, B.; Hulsman, L.; Zhang, Y.; Duvigneau, J.; Vancso, G. J. Nanocellular polymer foams nucleated by core-shell nanoparticles. Polymer 2016, 104, 22-30.

[9] Liu, S.; de Beer, S.; Batenburg, K. M.; Gojzewski, H.; Duvigneau, J.; Vancso, G. J. Designer core-shell nanoparticles as polymer foam cell nucleating agents: The impact of molecularly engineered interfaces. ACS Applied Materials \& Interfaces 2021, 13 (14), 17034-17045.

[10] Liu, S.; Yin, S.; Duvigneau, J.; Vancso, G. J. Bubble seeding nanocavities: multiple polymer foam cell nucleation by polydimethylsiloxane-grafted designer silica nanoparticles. ACS Nano 2020, 14 (2), 1623-1634. 
[11] Gebald, C.; Wurzbacher, J. A.; Tingaut, P.; Zimmermann, T.; Steinfeld, A. Amine-based nanofibrillated cellulose as adsorbent for $\mathrm{CO}_{2}$ capture from air. Environmental Science \& Technology 2011, 45 (20), 9101-9108.

[12] Xin, Q.; Ma, F.; Zhang, L.; Wang, S.; Li, Y.; Ye, H.; Ding, X.; Lin, L.; Zhang, Y.; $\mathrm{Cao}, \mathrm{X}$. Interface engineering of mixed matrix membrane via $\mathrm{CO}_{2}$-philic polymer brush functionalized graphene oxide nanosheets for efficient gas separation. Journal of Membrane Science 2019, 586, 23-33.

[13] Liu, S.; Eijkelenkamp, R.; Duvigneau, J.; Vancso, G. J. Silica-assisted nucleation of polymer foam cells with nanoscopic dimensions: impact of particle size, line tension, and surface functionality. ACS Applied Materials \& Interfaces 2017, 9 (43), 37929-37940.

[14] Liu, D.; Wu, Q.; Andersson, R. L.; Hedenqvist, M. S.; Farris, S.; Olsson, R. T. Cellulose nanofibril core-shell silica coatings and their conversion into thermally stable nanotube aerogels. Journal of Materials Chemistry A 2015, 3 (30), 1574515754 .

[15] Raabe, J.; de Souza Fonseca, A.; Bufalino, L.; Ribeiro, C.; Martins, M. A.; Marconcini, J. M.; Tonoli, G. H. D. Evaluation of reaction factors for deposition of silica $\left(\mathrm{SiO}_{2}\right)$ nanoparticles on cellulose fibers. Carbohydrate Polymers 2014, $114,424-431$.

[16] Zhao, Q.; Yang, W.; Zhang, H.; He, F.; Yan, H.; He, R.; Zhang, K.; Fan, J. Graphene oxide Pickering phase change material emulsions with high thermal conductivity and photo-thermal performance for thermal energy management. Colloids and Surfaces A: Physicochemical and Engineering Aspects 2019, 575, 42-49.

[17] Yang, K.; Kang, Y. Y.; Ahn, H. J.; Kim, D.-G.; Park, N. K.; Choi, S. Q.; Won, J. C.; Kim, Y. H. Porous boron nitride/polyimide composite films with high thermal diffusivity and low dielectric properties via high internal phase Pickering emulsion method. Journal of Industrial and Engineering Chemistry 2020, 82, 173-179.

[18] Yu, J.; Choi, H. K.; Kim, H. S.; Kim, S. Y. Synergistic effect of hybrid graphene nanoplatelet and multi-walled carbon nanotube fillers on the thermal conductivity of polymer composites and theoretical modeling of the synergistic effect. Composites Part A: Applied Science and Manufacturing 2016, 88, 79-85. 


\section{Summary}

Pickering emulsion templates provide highly promising alternatives to construct advanced, polymer-based, hybrid materials with new or enhanced functions. In this Thesis, Pickering emulsion templates were used to obtain polylactic acid (PLA) composites with well dispersed, fully biodegradable nanocellulose. The Pickering emulsion templating approach offers a versatile route to disperse hydrophilic CNCs, with and without surface modification, in the hydrophobic PLA matrix. The rheological, thermal, and mechanical properties of PLA/nanocellulose composites with different nanocellulose content up to $15 \mathrm{wt} \%$ are elucidated in detail. Furthermore, the enzymatic degradation of PLA/nanocellulose composites was studied using the lipase from Candida rugosa and proteinase $\mathrm{K}$ from Tritirachium album. The application area of the PLA/CNC composites as foamed insulating materials was broadened by using a $\mathrm{CO}_{2}$ batch process with relatively mild operating conditions. In a next step, we exploited modified CNC particles to prepare functional hybrid materials with designer colors. We used dyed-nanocellulose to color polymethyl methacrylate (PMMA). PMMA was selected for its high transparency. The thermal and mechanical properties of the corresponding-colored composites were thoroughly investigated. In addition, the colored PMMA composite possessed good transparency. Finally, phase change emulsion gels consisting of two types of phase change materials were constructed using the excellent stability of Pickering emulsion templates. The gels exhibit some distinctive properties, including high stability, nonflammability, shape memory, and good mechanical properties, and have been explored as an environmentally friendly building thermal management system in model experiments. The materials synthesized and studied in this Thesis prove the great potential of the Pickering emulsion approach to obtain advanced engineering materials for the next generation "green" plastics.

In Chapter 1, a short introduction to the topics, the motivation, and overview of the work described in this Thesis are presented.

A literature review concerning the general topics discussed in this Thesis is described in Chapter 2. Firstly, a basic introduction to Pickering emulsions is provided. Secondly, the preparation approaches of hybrid materials using Pickering 
emulsion templates are presented and discussed, including the solvent evaporation and polymerization methods. Finally, different physical morphologies of hybrid materials designed by Pickering emulsion templates are reviewed.

PLA is one of the most promising biodegradable thermoplastic polyesters to replace petroleum-derived polymers. This is ascribed to PLAs' easy processability, biocompatibility, and good mechanical properties. But some properties, such as PLAs' brittleness and low crystallization rate, limit its widespread application. Nanocellulose is often used to improve PLA properties due to its biodegradability and excellent mechanical properties. However, nanocellulose dispersion in a PLA matrix remains an issue to be tackled. In Chapter 3, the Pickering emulsion method was studied and introduced to disperse cellulose nanocrystals (CNC) in a PLA matrix. Compared to the composites prepared by other methods, the composites obtained using Pickering emulsions displayed an improved $\mathrm{CNC}$ dispersion. The mechanical and thermal properties of PLA were improved due to the well-dispersed CNC addition. Rheological evaluations of the PLA/CNC composites confirmed this conclusion.

In Chapter 4, a new preparation process of color PMMA is described. Dyednanocellulose was added to a PMMA matrix using the Pickering emulsion method to make colored PMMA. The dyed nanocellulose is still a good emulsifier for the Pickering emulsion. Besides improving the mechanical properties of PMMA, the well-dispersed dyed nanocellulose introduced homogeneous colors to PMMA without significantly decreasing the colored PMMA transparency. Using different reactive dyes attached to the CNCs, the color of the composites can be readily tuned. The intensity of the color can be adjusted by changing the nanocellulose content in PMMA.

In Chapter 5, the enzymatic degradation of PLA/CNC composites prepared by the Pickering emulsion method was investigated using the lipase from Candida rugosa and proteinase $\mathrm{K}$ from Tritirachium album. The PLA we used was not degraded by the lipase from Candida rugosa, but proteinase $\mathrm{K}$ was very efficient to enhance degradation kinetics. The $\mathrm{pH}$ and the ion concentration of the degradation medium influenced the rate of PLA decomposition. Furthermore, the addition of CNCs to the PLA matrix can significantly increase the rate of degradation.

Biodegradable PLA-based foams can replace petroleum-based foaming products. As is shown in Chapter 6, PLA/CNC composites were prepared by the Pickering emulsion masterbatch route to obtain composites with low CNC contents (i.e. below 5 wt.\%) to be used as nucleating agents in foaming. In addition, we prepared PLA/CNC composites with conventional melt blending. The composite films were 
foamed using a $\mathrm{CO}_{2}$ batch process under mild conditions. $\mathrm{CO}_{2}$ was selected as the blowing agent due to its cheap and "green" properties. The foaming results showed that the CNC dispersion had a significant influence on PLA foaming behavior. The addition of well-dispersed CNCs by the Pickering emulsion masterbatch route improved the PLA foam morphology due to the nucleating effect of the added CNCs. In addition, the CNCs increase the melt viscosity of the PLA, resulting in reduced foam cell expansion and better foam cell stabilization in the processing window studied.

As shown in Chapter 7, phase change gels consisting of paraffin and hydrated salts were constructed using water-in-oil Pickering emulsion templates. Fumed hydrophobic silica was used to stabilize the emulsion and to provide enough rigidity for the following polymerization process at $70{ }^{\circ} \mathrm{C}$. The paraffin in the continuous phase and the hydrophilic polymer chains in the dispersed phase provided dual protection to avoid the undesirable water loss of the hydrated salts. The gels displayed high stability and good water retention properties. After 500 thermal cycles, the gels exhibited essentially the same latent heat as the virgin gels. The gels were nonflammable and robust due to the nature of the hydrated salts. We successfully demonstrated the potential of these gels to be exploited in thermal building management systems.

Following the experimental work as described in the previous Chapters, Chapter $\mathbf{8}$ provides two directions for future work to yield designer functionalized particles to even further improve the properties of the composites or to give new functions to the composites. 


\section{Samenvatting}

Pickering-emulsietemplates bieden veelbelovende alternatieven voor het construeren van geavanceerde, op polymeren gebaseerde, hybride materialen met nieuwe of verbeterde functies. In dit proefschrift werden Pickering-emulsietemplates gebruikt om polylactic acid (PLA) composieten te verkrijgen met daarin goed gedispergeerde, volledig biologisch afbreekbare nanocellulose. De Pickeringemulsie-templating-aanpak biedt een veelzijdige route om hydrofiele cellulose nanokristallen (CNCs), met en zonder oppervlaktemodificatie, in de hydrofobe PLAmatrix te dispergeren. De reologische, thermische en mechanische eigenschappen van PLA/nanocellulose composieten met nanocellulose gehaltes tot 15 gew.\% worden in detail toegelicht. Verder werd het enzymatische afbreken van PLA/nanocellulose composieten bestudeerd met behulp van de lipase van Candida rugosa en proteïnase $\mathrm{K}$ van Tritirachium album. Het toepassingsgebied van PLA/CNC-composieten als geschuimde isolatiematerialen werd verbreed door gebruik te maken van een $\mathrm{CO}_{2}$ batch schuimproces met relatief milde procesomstandigheden. In een volgende stap hebben we gemodificeerde CNC-deeltjes gebruikt om functionele hybride materialen met designerkleuren te maken. We gebruikten pigment gemodificeerde nanocellulose om polymethylmethacrylaat (PMMA) te kleuren. PMMA werd geselecteerd vanwege de hoge transparantie. De thermische en mechanische eigenschappen van de verkregen gekleurde composieten werd grondig onderzocht. Bovendien hadden de gekleurde PMMA-composieten een goede optische transparantie. Ten slotte werden faseveranderingsemulsiegels bestaande uit twee soorten faseveranderingsmaterialen geconstrueerd met behulp van de uitstekende stabiliteit van Pickeringemulsietemplates. De emulsiegels vertonen een aantal onderscheidende eigenschappen, waaronder een hoge stabiliteit, niet-ontvlambaarheid, vormgeheugen en goede mechanische eigenschappen, en zijn onderzocht als een milieuvriendelijk temperatuursbeheersysteem voor gebouwen in modelexperimenten. De materialen die in dit proefschrift zijn gesynthetiseerd en bestudeerd, bewijzen het hoge potentieel van de Pickering-emulsiebenadering om geavanceerde technische materialen te verkrijgen voor de volgende generatie "groene" kunststoffen.

In Hoofdstuk 1 wordt een korte introductie van de onderwerpen, de motivatie en 
een overzicht van het werk gepresenteerd in dit proefschrift gegeven.

Een literatuur overzicht voor de algemene onderwerpen besproken in dit proefschrift is te vinden in Hoofdstuk 2. Allereerst wordt er een introductie gegeven voor Pickering emulsies. Ten tweede worden de vervaardigingsmethoden van hybride materialen op basis van Pickering emulsies, inclusief de oplosmiddelverdamping en polymerisatie methoden, beschreven en bediscussieerd. Uiteindelijk worden verschillende fysische morfologieën van hybride materialen ontworpen middels Pickering-emulsietemplates in een overzicht besproken.

PLA is een van de meest veelbelovende biologisch afbreekbare thermoplastische polyesters ter vervanging van op aardolie gebaseerde polymeren. Dit wordt toegeschreven aan de gemakkelijke verwerkbaarheid, bio-compatibiliteit en goede mechanische eigenschappen van PLA. Echter sommige eigenschappen, zoals de brosheid en lage kristallisatiesnelheid van PLA, beperken de wijdverbreide toepassing ervan. Nanocellulose wordt vaak gebruikt om PLA-eigenschappen te verbeteren vanwege de biologische afbreekbaarheid en uitstekende mechanische eigenschappen. Goede dispersie van nanocellulose in een PLA-matrix blijft echter een probleem dat moet worden aangepakt. In Hoofdstuk 3 werd de Pickering-emulsiemethode bestudeerd en geïntroduceerd om CNCs in een PLA-matrix te dispergeren. Vergeleken met de composieten die met andere methoden zijn bereid, vertoonden de composieten verkregen met de Pickering-emulsies een verbeterde CNC-dispersie. De mechanische en thermische eigenschappen van PLA werden verbeterd door de toegevoegde, goed verdeelde CNCs. Reologische metingen aan de PLA/CNC-composieten bevestigden deze conclusie.

In Hoofdstuk 4 wordt een nieuw bereidingsproces van gekleurd PMMA beschreven. Een pigment gemodificeerde CNC werd toegevoegd aan een PMMAmatrix met behulp van de Pickering-emulsiemethode om gekleurd PMMA te maken. De pigment gemodificeerde $\mathrm{CNC}$ is nog steeds een goede emulgator voor de Pickering-emulsie. Naast het verbeteren van de mechanische eigenschappen van PMMA, introduceerde de goed gedispergeerde, gekleurde nanocellulose homogene kleuren in PMMA zonder de transparantie van het gekleurde PMMA aanzienlijk te verminderen. Met behulp van verschillende reactieve pigmenten bevestigd aan de CNCs, kan de kleur van de composieten gemakkelijk worden afgestemd. De intensiteit van de kleur kan worden aangepast door het nanocellulosegehalte in PMMA te wijzigen.

In Hoofdstuk 5 werd de enzymatische afbraak van PLA/CNC-composieten 
verkregen met de Pickering-emulsiemethode onderzocht met behulp van de lipase van Candida rugosa en proteïnase K van Tritirachium album. Het door ons gebruikte PLA werd niet afgebroken door de lipase van Candida rugosa, maar proteïnase $\mathrm{K}$ bleek zeer efficiënt in het verbeteren van de afbraakkinetiek. De $\mathrm{pH}$ en de ionenconcentratie van het afbraakmedium beïnvloedden de snelheid van PLA-afbraak. Bovendien kan de toevoeging van CNCs aan de PLA-matrix de afbraaksnelheid aanzienlijk verhogen.

Biologisch afbreekbare schuimen op PLA-basis kunnen schuimproducten op basis van aardolie vervangen. Zoals in Hoofdstuk 6 wordt beschreven, werden PLA/CNCcomposieten bereid door de Pickering-emulsie-masterbatch-route om composieten te verkrijgen met een laag CNC-gehalte (d.w.z. minder dan 5 gew.\%) om te gebruiken als kiemvormers bij schuimvorming. Daarnaast hebben we PLA/CNC-composieten gemaakt met conventionele thermoplastische menging. De verkrgen composietfilms werden onder milde omstandigheden geschuimd met behulp van een $\mathrm{CO}_{2}$-batchproces. Er werd gekozen voor $\mathrm{CO}_{2}$ als blaasmiddel vanwege de lage kostprijs en "groene" eigenschappen. De schuimresultaten toonden aan dat de CNC-dispersie een significante invloed had op het schuimgedrag van PLA. De toevoeging van goed verdeelde $\mathrm{CNCs}$ door de Pickering-emulsiemasterbatch-route verbeterde de PLAschuimmorfologie vanwege het kiemvormende effect van de toegevoegde CNCs. Bovendien verhogen de CNCs de smeltviscositeit van het PLA, wat resulteert in verminderde schuimcelexpansie en betere schuimcelstabilisatie in het bestudeerde verwerkingsvenster.

Zoals getoond in Hoofdstuk 7, werden faseveranderingsgels bestaande uit paraffine en gehydrateerde zouten geconstrueerd met behulp van water-in-olie Pickering-emulsietemplates. Fumed hydrofobe silica werd gebruikt om de emulsie te stabiliseren en voldoende stijfheid te verschaffen voor het daaropvolgende polymerisatieproces bij $70{ }^{\circ} \mathrm{C}$. De paraffine in de continue fase en de hydrofiele polymeerketens in de gedispergeerde fase verschaften een dubbele bescherming om ongewenst waterverlies van de gehydrateerde zouten te voorkomen. De gels vertoonden een hoge stabiliteit en goede waterretentie-eigenschappen. $\mathrm{Na} 500$ thermische cycli vertoonden de gels in wezen dezelfde latente warmte als de vers verkregen gels. De gels waren onbrandbaar en robuust vanwege de aard van de gehydrateerde zouten. We hebben met succes het potentieel van deze gels aangetoond voor het gebruik ervan in thermische gebouwbeheersystemen.

In navolging van het experimentele werk zoals beschreven in de voorgaande hoofdstukken, biedt Hoofdstuk 8 twee richtingen aan voor toekomstig werk om 
designer functionele deeltjes te produceren welke de eigenschappen van composieten nog verder verbeteren of nieuwe functies introduceren. 


\section{Acknowledgements}

Looking back to my PhD study, it is hard to believe that two years have passed. Although most of the past two years have been spent under the coronavirus, this is still one of the most wonderful and unforgettable times in my life. Before finishing my Thesis, I would like to express my gratitude to all those who supported, encouraged and guided me through this challenging period of my life.

First of all, I would like to express my deepest gratitude and sincere appreciation to my esteemed supervisors Prof. Julius Vancso and Prof. Xiaofeng Sui for their insightful advice, continuous support, and patience during the course of my $\mathrm{PhD}$ degree. Their immense knowledge, plentiful experience and rigorous attitude towards science have encouraged and inspired me in all the time of my academic research and daily life. It is a great honor to be your student and be part of MTP/SPC group. I wouldn't have this opportunity to be a joint PhD student to study in two different countries without both your supports. Dear Julius, your advices and comments on my experiments, presentation, manuscript and Thesis impressed me a lot and taught me how to design my experiments wisely and properly, analysis my results precisely and present my research clearly and effectively. Dear Xiaofeng, your deep and distinct understanding to the projects inspired me a lot. Every time I meet some problems in my projects, you always can give me some wonderful ideas from different perspectives. Without your guidance and supports, the work described in this Thesis wouldn't be accomplished. I learnt a lot from both of you and I enjoyed working with you. I wish you all the best in the future.

I would like to offer my special thanks to my daily supervisor, Dr. Joost Duvigneau for his invaluable supervision, support and tutelage during the course of my $\mathrm{PhD}$ degree. Dear Joost, Thank you for your insightful comments, endless help and encouragements during these past two years. No matter how busy you are, you always found some time to have a discussion with me every week. I really got a lot of wonderful ideas, inspiration and encouragements from you in each discussion. Your professional and rigorous attitude towards scientific research and writing inspired me a lot and it also will have an important impact on my future career. I am so fortunate and grateful working with you. I wish you all the best and successful in your future.

I really appreciate the opportunity to collaborate with Prof. Béla Pukánszky and your wonderful PhD students, Dr. Nora Hegyesi and Lu Cui. Your professional 
knowledge and advice on the research of polylactic acid/nanocellulose composites helped us to deeply analyze and evaluate the properties of the obtained composites. The degradation project we cooperated made me have a deep understanding about polylactic acid degradation process. Thank you so much!

I would like to extend my sincere thanks to Clemens Padberg, a wonderful lab manager in our group. When I first came to MTP/SPC, the words I heard the most is "asking Clemens". The facts showed that Clemens can help you to fix all the problems that you meet in the lab. SEM is the main characterization for my research. Whenever I need to do SEM, you are always willing and glad to help me. Thank you for your numerous SEM images with high quality, all training of equipment and chemical orders. I also would like to thank Frederik, Sissi, Hubert, Mark, Ramon, Michel and Olivier for their kind help during my PhD study. I also would like to express many thanks to Marion, Izabel and Nicole, the secretaries of the group, for all your enormous help with all type of paperwork and documents. Jirka, I really enjoyed the time working with you. You are a so nice student. I wish you all the best in your $\mathrm{PhD}$ study and future life!

I would also like to express my warm and sincere thanks to my graduation committee members, Prof. dr. ir. R. G. H. Lammertink, Prof. dr. ir. R. ten Klooster, Prof. dr. B. Pukánszky and Prof. dr. ir. R. Tuinier, for their invaluable advices on my Thesis and being my committee members. I wish you all the best in the future!

Next, I would like to give my sincere thanks to my nice colleagues, Georgios, Matthijs, Roland and Diego for their help in lab and daily life. Georgios, I really enjoyed the talks with you. I still remember when I came to the Netherlands and my English sucked, you still talked to me every day, explained your project to me and help me to solve the problems of my project with endless patience. Thanks a lot! I can never forget the delicious coffee you cooked and the nice cookie you bring every week. I hope to see you in China someday. I wish you all the best and successful in your PhD study and future life. Matthijs, thank you for your help on the PLA foaming work. You taught me a lot when I first started this work to avoid some troubles. I also enjoyed the talks with you. I wish you all the best in your job and future life! Roland, thank you for your help in lab work. I really like the Wednesday Pizza Party you and Georgios organized. I wish you all the best and successful in your $\mathrm{PhD}$ study and future life. Diego, I really enjoyed the talks with you. Thank you for explaining your project to me with patience! Your project is very interesting. I wish you all the best and successful in your postdoc life in Oxford university! 
I would like to express my special thanks to my Chinese friends, Huan, Yan, Sida, Jinmeng, Min, Lijie, Wenxing and Sishi. Yan, I can still remember the scene that you and Marco picked me, Huan and Liduo up at Enschede train station on my first day in the Netherlands. Thank you so much for your endless and invaluable help when I first came here. Huan, we were master classmate, $\mathrm{PhD}$ classmate and then came to the Netherlands together. Thank you so much for your invaluable help during my master and PhD life! Dear all my best friends, thanks a lot for your help in MTP/SPC and my daily life. I really enjoyed the time we spent together. I can never forget the delicious "lamb hot pot" and interesting "You draw I guess" game. I wish you all the best in your PhD project and future life! Shanqiu, a former MTP member, thank you for your help and suggestion on my project when I first came here.

I would like to express my thanks to the group members in Donghua University, Prof. Zhiping Mao, Prof. Hong Xu, Prof. Xueling Feng, Dr. Bijia Wang, for all your invaluable comments on my project and manuscript. Dr. Yang Jiang, Dr. Yingzhan Li, Dr. Lingli Liu, Dr. Xiang Li, Dr. Liduo Rong, Dr. Lei Ding, Feifei Wang, Yan Wang, Yating Wang, Yunbo Chen, for all your help in my project and daily life. I wish you all the best in your future!

Finally, I would like to express my deepest gratitude to my families. I am very grateful to my father (张利军), my mother (翟翠芹), my grandfather (张凤岐), my grandmother (宋贵云), my brother (张云航) and my sister (张晓宇) for all your endless love, supports and encouragement. I regret not spending much time with you together over the past years. I will try to spend much more time with all you in the future. I would express my special and deepest love and gratitude to my wife (宋晓 月), this is the tenth year we have been together. Word is powerless to show my thanks to you for your love, support, tolerance and encouragement. You mean too much to me. In the past ten years, we spent much more time apart than together, from two different cities to two different provinces to two different countries. It is your understanding and dedication that made me today. I feel very lucky that I've you as my life partner. Thanks for being the most amazing wife in the world. I promise we will never be separated anymore and try my best to give you the warmest family in the world. I also would like to thank my parents-in-law for your help and support.

张云崇

Yunchong Zhang

Enschede and Shanghai, 2021 


\section{Publications:}

\section{$>$ Journal articles:}

1. Zhang, Y., Ding, L., Ming, B., Wang, B., Feng, X., Vancso, G. J.*, \& Sui, X.*, Making polymers colored and stiffer by dyed regenerated cellulose employing Pickering emulsions. Colloids and Surfaces A: Physicochemical and Engineering Aspects, 2020, 592, 124601.

2. Zhang, Y., Cui, L., Xu, H., Feng, X., Wang, B., Pukánszky, B.*, Mao, Z., \& Sui, X.*, Poly (lactic acid)/cellulose nanocrystal composites via the Pickering emulsion approach: Rheological, thermal and mechanical properties. International Journal of Biological Macromolecules, 2019, 137, 197-204.

3. Hegyesi, N., Zhang, Y., Kohári, A., Polyák, P., Sui, X.*, \& Pukánszky, B.*, Enzymatic degradation of PLA/cellulose nanocrystal composites. Industrial Crops and Products, 2019, 141, 111799.

4. Baraki, S. Y., Zhang, Y., Li, X., Ding, L., Debeli, D. K., Macharia, D. K., Wang, B., Feng, X., Mao, Z., \& Sui, X.*, Regenerated chitin reinforced polyhydroxybutyrate composites via Pickering emulsion template with improved rheological, thermal, and mechanical properties. Composites Communications, $2021,25,100655$.

5. Wang, F., Zhang, Y., Li, X., Wang, B., Feng, X., Xu, H., Mao, Z., \& Sui, X.*, Cellulose nanocrystals-composited poly (methyl methacrylate) encapsulated $n$ eicosane via a Pickering emulsion-templating approach for energy storage. Carbohydrate Polymers, 2020, 234, 115934.

6. Munyazesa, F. X., Zhang, Y., Wang, B., Wang, C., Feng, X., Mao, Z., Chen, Y., $\&$ Sui, X.*, Pickering emulsion process assisted construction of regenerated chitin reinforced poly (lactic acid) blends. International Journal of Biological Macromolecules, 2019, 140, 10-16.

7. Jiang, Y., Zhang, Y., Ding, L., Joshua, A., Wang, B., Feng, X., Chen, Z., Mao, Z $\&$ Sui, X.*, Regenerated cellulose-dispersed polystyrene composites enabled via Pickering emulsion polymerization. Carbohydrate Polymers, 2019, 223, 115079. 
8. Lugoloobi, I., Li, X., Zhang, Y., Mao, Z., Wang, B., Sui, X.*, \& Feng, X., Fabrication of lignin/poly (3-hydroxybutyrate) nanocomposites with enhanced properties via a Pickering emulsion approach. International Journal of Biological Macromolecules, 2020, 165, 3078-3087.

9. Zeng, M., Li, X., Zhang, Y., Chen, X., Sui, X.* , \& Yuan, J.*, Tailoring the droplet size of Pickering emulsions by PISA synthesized polymeric nanoparticles. Polymer, 2020, 206, 122853.

10. Chai, H., Chang, Y., Zhang, Y., Chen, Z., Zhong, Y., Zhang, L., Sui, X., Xu, H.*, \& Mao, Z.*, The fabrication of polylactide/cellulose nanocomposites with enhanced crystallization and mechanical properties. International Journal of Biological Macromolecules, 2020, 155, 1578-1588.

11. Li, X., Ding, L., Zhang, Y., Wang, B., Jiang, Y., Feng, X., Mao, Z., \& Sui, X.*, Oil-in-water Pickering emulsions from three plant-derived regenerated celluloses. Carbohydrate Polymers, 2019, 207, 755-763.

12. Li, X., Hegyesi, N., Zhang, Y., Mao, Z., Feng, X., Wang, B., Pukánszky, B.*, \& Sui, X.*, Poly (lactic acid)/lignin blends prepared with the Pickering emulsion template method. European Polymer Journal, 2019, 110, 378-384.

13. Cui, L., Yi, L., Wang, Y., Zhang, Y., Polyák, P., Sui, X.*, \& Pukánszky, B.*, Rheology of PLA/regenerated cellulose nanocomposites prepared by the Pickering emulsion process: Network formation and modeling. Materials $\boldsymbol{\&}$ Design, 2021, 206, 109774.

14. Ding, L., Li, X., Hu, L., Zhang, Y., Jiang, Y., Mao, Z., Xu, H., Wang, B., Feng, $\mathrm{X}$., \& Sui, $\mathrm{X}^{*}$., A naked-eye detection polyvinyl alcohol/cellulose-based $\mathrm{pH}$ sensor for intelligent packaging. Carbohydrate Polymers, 2020, 233, 115859.

\section{$>$ Book chapter:}

1. Zhang, Y., Cheng, H., Li, X., Wang, B., Feng, X., Mao, Z., Vancso, G.J.*, and Sui, X.*, Pickering emulsions as designer platforms for polymer-based hybrid materials: Routes to controlled structures, Macromolecular Engineering, John Wiley \& Sons Ltd, accepted and in production 


\section{$>$ Manuscripts submitted:}

1. Zhang, Y., Wang, F., Duvigneau, J., Wang, Y., Wang, B., Feng, X., Mao, Z., Vancso, G.J.*, and Sui, X.*, Highly stable and non-flammable hydrated saltparaffin shape-memory gels for sustainable building technology.

2. Zhang, Y., Duvigneau, J.*, Sui, X.*, and Vancso, G.J.*, Foaming behavior of biodegradable amorphous poly (lactic acid)/cellulose nanocrystal composites prepared by the Pickering emulsion masterbatch route.

\section{$>$ Other publications:}

1. Zhang, Y., Jiang, Y., Han, L., Wang, B., Xu, H.*, Zhong, Y., Zhang, L., Mao, Z., \& Sui, X.*, Biodegradable regenerated cellulose-dispersed composites with improved properties via a pickering emulsion process. Carbohydrate Polymers, 2018, 179, 86-92.

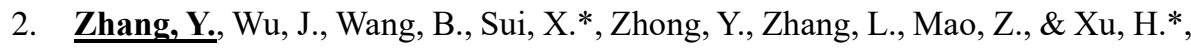
Cellulose nanofibril-reinforced biodegradable polymer composites obtained via a Pickering emulsion approach. Cellulose, 2017, 24(8), 3313-3322.

3. Jiang, Y., Zhang, Y., Ding, L., Wang, B., Chen, Z., Zhong, Y., Zhang, L., Mao, Z., Xu, H., \& Sui, X.*, Sag control of waterborne acrylic latex with regenerated nanocellulose suspension. Progress in Organic Coatings, 2018, 123, 146-152.

4. Han, L., Wang, B., Dai, Y., Zhang, Y., Xu, H., Sui, X., Zhang, L., Zhong, Y., \& Mao, Z.*, Dually self-reinforced Poly ( $\varepsilon$-caprolactone) composites based on unidirectionally arranged fibers. Composites Science and Technology, 2018, 165 , 331-338. 


\section{Curriculum vitae}

Yunchong Zhang was born on $11^{\text {th }}$ August 1992 in Xingtai, Hebei province, China. In September 2011, he entered Hebei University of Science \& Technology for his bachelor degree. In June 2015, he obtained his bachelor degree in Chemical Engineering with a focus on chemical dyeing under the supervision of Prof. Haiyan Zhang. In the same year, he started his master studies in College of Chemistry, Chemical Engineering and Biotechnology of Donghua University under the supervision of Prof. Hong Xu and Prof. Xiaofeng Sui. His project focused on preparation of poly(lactic acid)/nanocellulose nanocomposites based on the Pickering emulsion templating method. After graduation in 2018, he continued as a joint $\mathrm{PhD}$ candidate under the supervision of Prof. G. Julius Vancso, Prof. Xiaofeng Sui and Dr. Joost Duvigneau between University of Twente and Donghua University. His project focused on designing polymer-based hybrid materials using Pickering emulsion templating method and studying their performance. The results of his research are described in this Thesis. 


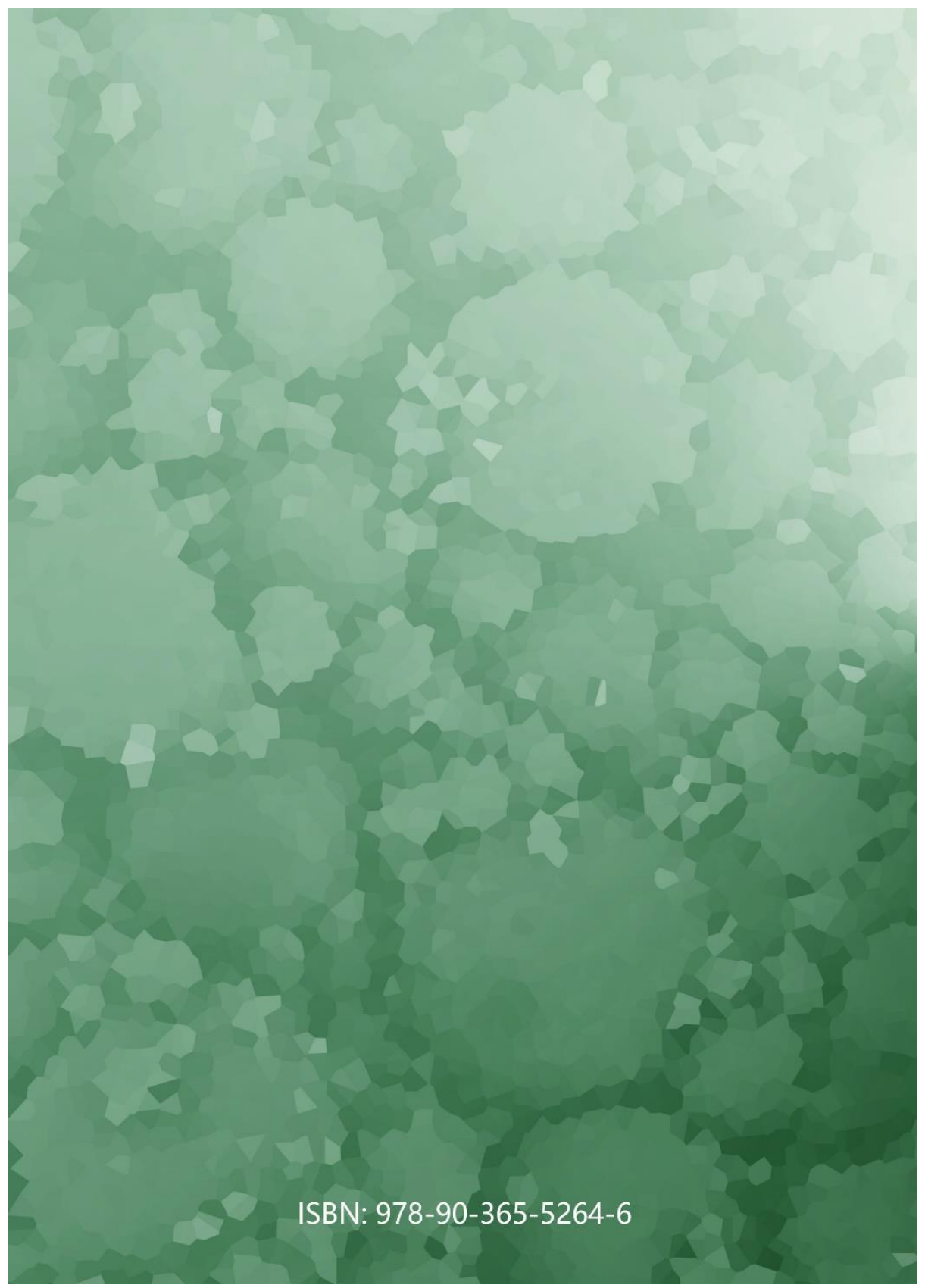

